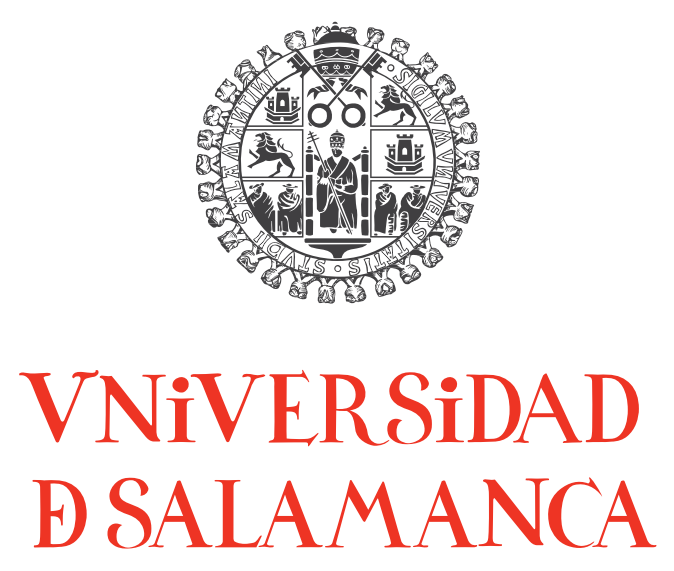

Departamento de QUÍMICA FÍSICA

\title{
Stereodynamics of elementary reactions: Effect of the reagent's rotational angular momentum polarisation
}

Jesús J. Aldegunde Carrión

Salamanca, 2007 


\section{Stereodynamics of elementary reactions: Effect of the reagent's rotational angular momentum polarisation}

Memoria presentada por el Licenciado

Jesús José Aldegunde Carrión para optar al Grado de Doctor en Química.

Salamanca, Marzo de 2007 
José María Alvariño Herrero, Catedrático de Química Física en la Universidad de Salamanca, Francisco Javier Aoiz Moleres, Catedrático de Química Física en la Universidad Complutense de Madrid y Marcelo Pessoa de Miranda, Lecturer en la Universidad de Leeds

CERTIFICAN que el trabajo descrito en la presente memoria "Stereodynamics of elementary reactions: Effect of the reagent's rotational angular momentum polarisation" ha sido realizado bajo su dirección por el Licenciado en Ciencias Químicas D. Jesús José Aldegunde Carrión y

AUTORIZAN su presentación en los términos previstos en la legislación vigente.

Salamanca, 29 de Marzo de 2007

Fdo. José María Alvariño Herrero

Fdo. Francisco Javier Aoiz Moleres

Fdo. Marcelo Pessoa de Miranda 
Este trabajo ha sido llevado a cabo en el Departamento de Química Física de la Universidad de Salamanca, en el Departamento de Química Física I de la Universidad Complutense de Madrid y en la School of Chemistry de la Universidad de Leeds bajo la dirección de los Profs. Dr. José María Alvariño Herrero, Dr. Francisco Javier Aoiz Moleres y Dr. Marcelo Pessoa de Miranda, a quienes expreso mi más sincero agradecimiento por su constante ayuda, apoyo y paciencia.

Así mismo, hago extensivo mi agradecimiento a todos los miembros del Departamento de Química Física de la Universidad de Salamanca y, en particular, al Prof. Tel, con quien compartí tantas interesantes discusiones.

Gracias a la Dra. Mari Luz Hernández y al Dr. Vicente Saéz Rábanos, por su disponibilidad y ayuda durante estos cuatro años.

A David y Cristina, con quienes disfruté de muchos buenos (y no tan buenos) cafés.

Agradezco el uso de los medios materiales de los proyectos de investigación BQU2002-04462-C02-01 y CTQ2005-09185-C02-02 de la DGI, así como la beca predoctoral FPU-AP2002-0594 de la que fui beneficiario.

A mi familia, pieza fundamental de mi engranaje vital y sin quienes nada de esto hubiera sido posible. Por soportarme, sobre todo al final. 
Para Anastasio 


\section{Contents}

1. Introduction 5

2. Polarisation moments 11

2.1. Probability density function and classical polarisation moments . . . . 12

2.1.1. Probability density function (classical PDF) . . . . . . . . . . . 12

2.1.2. Classical polarisation moments . . . . . . . . . . . . . 13

2.2. Density matrix and quantum mechanical polarisation moments . . . . 13

2.2.1. Density operator and density matrix . . . . . . . . . . . . . 14

2.2.2. Quantum mechanical polarisation moments . . . . . . . . . 19

2.3. Polarisation moments for special systems . . . . . . . . . . . . . . . . . 23

2.3.1. Axially symmetric systems . . . . . . . . . . . . . . 24

2.3.2. Systems with a plane of symmetry . . . . . . . . . . . 25

2.3.3. Unpolarised systems . . . . . . . . . . . . . . . . 27

2.4. Directional interpretation of the polarisation moments . . . . . . . . . 28

2.4.1. Real polarisation moments . . . . . . . . . . . . . 29

2.4.2. Meaning of the real polarisation moments . . . . . . . . . . . 30

2.4.3. Renormalised polarisation moments . . . . . . . . . . . 31

2.5. Classical and quantum-mechanical moments comparison. Quantum PDF. 32

2.5.1. Quantum population distribution function . . . . . . . . 33

3. Atom-diatom collisions 39

3.1. Cross section . . . . . . . . . . . . . . . . . 40

3.2. Born-Oppenheimer approximation . . . . . . . . . . . . 43

3.3. Quasiclassical trajectory method . . . . . . . . . . . . . . 48

3.4. Quantum reactive scattering . . . . . . . . . . . . . . 52

4. Reaction control and mechanism analysis through the reactants polarisa-

$\begin{array}{ll}\text { tion } & 67\end{array}$ 
4.1. Intrinsic and extrinsic properties of chemical reactions $\ldots \ldots \ldots 68$

4.2. Intrinsic and extrinsic reactants polarisations . . . . . . . . . . 69

4.3. Intrinsic polarisation moments . . . . . . . . . . . . 70

4.3.1. State-to-state PDDCSs . . . . . . . . . . . . . . 71

4.3.2. State-to-state PPs . . . . . . . . . . . . . . . . 74

4.3.3. Total PDDCSs . . . . . . . . . . . . . 76

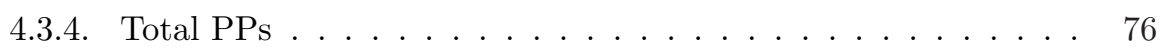

4.3.5. Calculation of the state-to-state PDDCSs . . . . . . . 76

4.4. Reaction observables dependence on the $\mathbf{j}$ polarisation . . . . . . 78

4.4.1. Observable state-to-state differential cross section . . . . . . . 79

4.4.2. Observable state-to-state integral cross section . . . . . . . . 81

4.4.3. Total observables . . . . . . . . . . . . . . . . . . . . . 82

4.4.4. Enhancement numbers . . . . . . . . . . . . . . . . . . . 83

4.5. Stereodynamical portraits . . . . . . . . . . . . . . . 90

4.5.1. Quantum mechanical portraits . . . . . . . . . . . . . 91

4.5.2. Quasiclassical portraits . . . . . . . . . . . . . 93

4.6. Experimental feasibility of the proposed measurements . . . . . . . 94

4.7. Illustrative examples . . . . . . . . . . . . . . . . . . 100

4.7.1. The intrinsic approach: insight and understanding . . . . . . 101

4.7.2. The practical approach: experimental control possibilities . . . 106

4.7.3. The min-max approach: theoretical control limits . . . . . . . 114

5. Mechanism of the $\mathbf{H}+\mathrm{D}_{2}$ reactive collisions 121

5.1. Direct and delayed mechanisms . . . . . . . . . . . . . . 122

5.2. Total integral results . . . . . . . . . . . . . . . . . . 123

5.3. State-to-state integral results . . . . . . . . . . . . . . . 127

5.4. Total differential results . . . . . . . . . . . . . . . . . . 130

5.5. State-to-state differential results $\ldots \ldots \ldots \ldots \ldots$

6. Control and mechanism of the $\mathrm{F}+\mathrm{H}_{2}$ reactive collisions 141

6.1. Reaction general features . . . . . . . . . . . . . . . . 142

6.2. Reactive scattering calculations . . . . . . . . . . . . . . . 144

6.3. Vibrationally resolved integral results . . . . . . . . . . . 145

6.4. State-to-state integral results . . . . . . . . . . . . . . 147

6.5. Vibrationally resolved differential results . . . . . . . . . . . . . 153

6.6. State-to-state differential results . . . . . . . . . . . . . . . . 161 
7. Mechanism and control of atom-diatom reactions at low and ultralow col$\begin{array}{ll}\text { lision energies } & 169\end{array}$

7.1. Cold and ultracold reactions . . . . . . . . . . . . . . . . . . 170

7.2. Control of $\ell=0$ reactions . . . . . . . . . . . . . . . . . 171

7.2.1. Ultracold reactions and $\boldsymbol{S}$ matrix . . . . . . . . . . . 171

7.2.2. PPs and PDDCSs for ultracold reactions . . . . . . . . . 172

7.2.3. Reaction control in the ultracold limit . . . . . . . . . . . . 175

7.3. $\mathrm{F}+\mathrm{H}_{2}$ reaction stereodynamics at low and ultralow collision energies . 175

7.3.1. Reactive scattering calculations . . . . . . . . . . 176

7.3.2. Results . . . . . . . . . . . . . . . 176

$\begin{array}{ll}\text { 8. Conclusions } & 187\end{array}$

Appendixes

\begin{tabular}{ll}
\hline A. Rotations in quantum mechanics & 191
\end{tabular}

$\begin{array}{ll}\text { B. Real polarisation moments } & 197\end{array}$

$\begin{array}{ll}\text { C. Observable DCS } & 205\end{array}$

\begin{tabular}{ll}
\hline D. Molecular axis portraits & 209
\end{tabular}

\begin{tabular}{ll}
\hline E. Derivation of Eq.(7.18) & 211
\end{tabular}

F. Estereodinámica de reacciones elementales: efecto de la polarización del momento angular rotacional de los reactivos 213

F.1. Introducción . . . . . . . . . . . . . . . . . . . . . . . . . . 213

F.2. Teoría . . . . . . . . . . . . . . . . . . . . . . . . . 214

F.2.1. Método clásico . . . . . . . . . . . . . . . . . . . 214

F.2.2. Método mecano-cuántico . . . . . . . . . . . . . . . . 218

F.2.3. Retratos estereodinámicos . . . . . . . . . . . . . . . 220

F.3. Ejemplos y resultados . . . . . . . . . . . . . . . . . . . . 221

F.3.1. Determinación experimental de la influencia de la polarización de los reactivos . . . . . . . . . . . . . . . . . 221

F.3.2. Reacción $\mathrm{D}+\mathrm{H}_{2}(v=0, j=2) \ldots \ldots \ldots \ldots 223$

F.3.3. Reacción $\mathrm{F}+\mathrm{H}_{2}(v=0, j=2) \ldots \ldots \ldots \ldots 231$

F.3.4. Reacciones ultrafrías . . . . . . . . . . . . . . . . 233

F.4. Conclusiones . . . . . . . . . . . . . . . . . . . 237 
Bibliography 


\section{Introduction}

Two of the most persistent goals of scientific investigations of the dynamics of molecular collisions are understanding and control [1, 2]. On the one hand, collision dynamicists strive for a detailed understanding of collision mechanisms and of the role of energetic and directional factors in scattering events [3, 4, 5]. On the other hand, they attempt to devise techniques for the control of molecular collisions and, in particular, for the selection of desired collision outcomes $6,7,8$. Naturally, the two endeavours go hand in hand. Understanding of collision mechanisms facilitates the development of control schemes, while analysis of the dynamics of controlled collisions can offer important clues about collision mechanisms.

Both issues are directly related to the subject matter of this work: to study the stereodynamics 1 of atom-diatom reactions

$$
\mathrm{A}+\mathrm{BC} \rightarrow \mathrm{AB}+\mathrm{C}
$$

through the consideration of the role of the BC rotational angular momentum (j) polarization in the dynamics of the process. This analysis is performed by means of theoretical tools useful (i) for the analysis of the dependence of reaction mechanisms on reactant polarization, and (ii) for the selection of optimum reactant polarization schemes for the control of reaction probabilities and product state distributions.

The techniques and results presented throughout the manuscript contribute to fill the gap existing between the quite obvious idea that collision geometry can influence chemical reactivity and the exploration of stereodynamics with rigorous and quantitative theory. It is often the case that detailed studies of the stereodynamics of molecular collisions lead to surprising, counterintuitive results. A particularly striking example is provided by the Li + HF system. Quantum theoretical studies [17, 18] of this reaction at zero total angular momentum and with HF in its ground vibrational state have confirmed the earlier predictions of QCT calculations [19], namely that formation of the $\mathrm{LiF}$ product is favoured not only when the Li atom attacks the $\mathrm{F}$ end of HF, as one would intuitively expect, but also (and perhaps more strongly)

\footnotetext{
${ }^{1}$ Reaction dynamics explicitly accounting for stereochemical factors $9,10,11,12,13,14,15,16$
} 
when the $\mathrm{Li}$ atom attacks the $\mathrm{H}$ end of HF. Experimental studies of the same reaction by Loesch and coworkers [20, 21], this time involving $\operatorname{HF}(v=1)$ molecules, have confirmed steric effects to be important, but in a rather different manner. The detailed analysis of their data was carried out in the light of wavepacket [22] and QCT [23] calculations, and the theoretical studies have lead to the conclusion that steric effects are actually quite unimportant for the integral reaction cross section summed over product states, but much more significant for differential properties and for the product state distribution, with formation of LiF in its $v^{\prime}=0$ or $v^{\prime}=3$ vibrational states being favoured, respectively, by head-on or side-on Li + HF collisions [23].

Two versions of the method will be presented: one classical, appropriate for use in conjunction with quasiclassical trajectory (QCT) calculations, the other quantum mechanical, appropriate for use in conjunction with time independent quantum scattering calculations. Both versions are strongly based on quantum 24, 25] and classical [25] methods used before for the description of polarization effects in the dynamics of atom-diatom reactions, which in turn were strongly based on previous vector correlation theories [26, 27, 28, 29, 30, 31, 32]. The quantum and classical theories have been stated using analogous formalisms, and revising or extending some of the definitions used in the past in order to maximize the chemical insight that can be gained by analysis of the calculable (and observable) quantities, and in order to facilitate the comparison between (possibly experimental) quantum and classical results.

With the purpose of encouraging experimental approaches to the problem, a currently feasible experiment capable of probing the phenomena theoretically addressed is also described. Such experiment consists of aligning the BC rotational angular momentum $\mathbf{j}$ (and thus the internuclear axis $\mathbf{r}$ ) perpendicular to (along) a laboratory fixed axis whose position in the centre of mass reference frame can be controlled so that different arrangements of the axis will correspond to different collision geometries. Theoretical predictions for possible outcomes of the experiment are widely employed throughout the work because, on top of providing with an estimation of the control achievable through the reactants polarisation, they can be intuitively interpreted in terms of the reaction mechanism.

The dichotomy of intrinsic and extrinsic reaction properties lies on the basis of the methods presented. The former describe the reactive process itself and are inherently determined by the collision dynamics rather than by external circumstances. The second group of properties describes the experiment employed to study the reaction and, consequently, they can be externally modified.

In order to further clarify the difference between both kinds of properties, let us 
consider the following analogy. Suppose we are inside a Gothic cathedral. The light we see depends not only on the quality of light but on the motifs and colours of the stained-glass windows. Their different pieces filter and alter the sunlight in such a way that we cannot perceive that light but only the components that can go through the windows. In a chemical reaction, the intrinsic properties play the role of the sunlight, the experiment characterised by the extrinsic properties plays the role of the filter, that is, of the coloured glass pieces and, finally, the light we perceive into the cathedral represents the experiment output. The intrinsic properties are inherent to the reaction and they only reveal their presence by means of an experiment, whose output represents the way in which we perceive them. Of course, the output changes as we change the experimental conditions (the glass pieces of the window), but this change does not affect the intrinsic nature of the reaction, it only affects the way in which we perceive it. Consequently, the observable quantities related to the reaction are simultaneously determined by both intrinsic and extrinsic properties, that is, by the nature of the reaction and by the characteristics of the experiment employed to probe such nature.

Not all the reaction properties are equally useful from the point of view of unveiling the stereodynamics of chemical reactions. In particular, it is convenient to consider the polarisation of the $\mathrm{BC}$ rotational angular momentum and to emphasise the differences between the intrinsic and the extrinsic polarisations. The former represents the reactants polarisation that gives rise to reaction into a given final state, at a given scattering angle and, possibly, with a defined polarisation of the products. The intrinsic polarisation contains the directional preferences of the reaction and can not be altered by any external procedure. In turn, the extrinsic polarisation corresponds to the actual (and necessarily particular) preparation of the rotational angular momentum of the reactants when an experiment takes place, and can be externally determined.

The reaction output is determined by the merging of the intrinsic and extrinsic polarisations, being possible to selectively modify the reaction probability by preparing the reactants prior to collision according to different extrinsic polarisations. As the effect of a certain experimental polarisation on the reaction probability will depend, in general, on the products internal state and the products recoil direction, it will be feasible to control not only the reaction probability but also the products states distribution and the differential cross section.

Quantification of the extent of this control is based on the consideration of polarisation moments $[29,31,33$. These are numeric parameters that mathematically represent the polarisation of any angular momentum. In particular, our interest will 
focus on the intrinsic and extrinsic polarisation moments holding for the intrinsic and the extrinsic reactants polarisations. The intrinsic polarisation moments play a twofold role: they quantify the directional preferences of the reaction and relate the $\mathbf{j}$ polarisation to reaction probabilities. The extrinsic moments numerically describe the experimental reactants preparation. It will be found that the reaction cross sections can be expressed in terms of both sets of moments through expressions that, when employed, allow for evaluating the change in the observables values caused by preparing the reactants in a certain manner, that is, allow for quantifying the control achievable by extrinsically polarising the reactants.

On top of this, the values of the intrinsic moments furnish us with a measurement of $\mathbf{j}$ alignment ${ }^{2}$ or orientation ${ }^{3}$ with respect to well defined directions in the space when the reaction happens. Therefore, the reaction mechanism can be unveiled by analysing the directional information contained in each of the moments. This procedure, however, poses a difficulty: it is necessary to merge the information contained in all the intrinsic moments in order to obtain a global view of the mechanism. The accomplishment of this task involves a considerable mental effort and, if the number of moments is too high, becomes unattainable. Fortunately, this difficulty can be overcome by using the stereodynamical portraits: three dimensional distributions which condense the intrinsic moments information and represent the $\mathbf{j}$ and $\mathbf{r}$ arrangements leading to products formation. The employment of stereodynamical portraits will be proved to supply with the connection between the time independent scattering calculations and the mechanism language, constituting a powerful visual tool for probing the reaction mechanism.

The work is organized as follows. The theory presentation starts with Chapters 2 and 3. The former includes the classical and quantum mechanical definitions of the polarisation moments, together with their main properties. The second is devoted to scattering theory, providing with a general description of the different procedures used for tackling with the study of reactive collisions. Working on the basis of Chapters 2 and 3, Chapter 4 completes the theoretical part of the work by including (i) the introduction of the extrinsic and the intrinsic reactants polarisations and explicit expressions for the evaluation of the intrinsic moments, (ii) expressions for the reaction cross sections when the reactants are arbitrarily polarised, (iii) a discussion about the extent of the reaction control achievable through such polarisation and about the experimental feasibility of measuring that control, (iv) the presentation of the stere-

\footnotetext{
${ }^{2}$ An angular momentum $\mathbf{j}$ is said to be aligned when it displays a preference for being directed along a certain spatial direction

${ }^{3}$ An angular momentum $\mathbf{j}$ is said to be oriented when, beyond displaying a preference for being directed along an spatial direction $(z)$, it shows as well a bias towards $+z$ or $-z$
} 
odynamical portraits and (v) numerical examples that employ the $\mathrm{H}+\mathrm{D}_{2}(v=0, j=2)$ reaction to illustrate the former points. The following Chapters make use of the tools devised in Chapter 4 to cope with different problems: Chapter 5 analyzes the mechanism of the $\mathrm{H}+\mathrm{D}_{2}$ reactive collisions through the information contained in the intrinsic polarisation, Chapter 6 studies the mechanism and control of the $\mathrm{F}+\mathrm{H}_{2}$ reaction and Chapter 7 tackles the control and mechanism of ultracold collisions, proving that control through the reactants polarisation is feasible in the ultracold limit. Finally, Chapter 8 closes the work with a summary of the main results and conclusions. In order to make easier to get the gist of the dissertation, some mathematical derivations are moved into Appendices placed at the end of the manuscript. 


\section{Polarisation moments}

To be able of representing mathematically the polarisation of an angular momentum is a "sine qua non" requirement to analyse the directional effects related to any chemical reaction. The scope of the present Chapter is to provide with a brief summary of the mathematical tools that make possible to fulfill this goal.

Let us consider a physical system with $\mathbf{j}$ angular momentum $1 \frac{1}{}$. Two alternative descriptions of the $\mathbf{j}$ polarisation will be presented: one based on classical mechanics and another one based on quantum mechanics. While the former does not set any constrain to the simultaneous knowledge of the three cartesian components of the angular momentum, in the quantum mechanical frame it is necessary to consider the uncertainty principle which sets that only the modulus and one of the components can be simultaneously determined [34, 35].

This upper limit to the knowledge of the $\mathbf{j}$ direction will be the origin of the dissimilarities between both descriptions. They will have in common that the polarisation features will be expressed by means of a set of quantities called "polarisation moments" that represent the coefficients of a multipolar expansion [25, 36]. However, the identity of the expanded function will be different as we use classical or quantum mechanics: in the former case it will be the classical probability density function (PDF) of finding the angular momentum pointing along a defined spatial direction while, in the second, the elements of the density matrix describing the state of the system will be employed.

Although the classical PDF and the density operator contain by themselves all the information regarding the polarisation of the system angular momentum, the use of a multipolar expansion will imply some benefits. On the one hand, we split all that information in small pieces (the polarisation moments) which are (i) easier to "digest" and interpret than the whole bulk and (ii) experimentally measurable. On the other hand, we will see that the polarisation moments behave as spherical tensors [37, 38] under rotations. This will make possible to gain maximum advantage by using the symmetry properties of the system under study.

\footnotetext{
${ }^{1}$ In the present Chapter $\mathbf{j}$ will represent a generic angular momentum and not necessarily the rotational angular momentum of a diatomic molecule, as it will be the case in the rest of the manuscript
} 
The classical and quantum mechanical mathematical descriptions of the polarisation moments are presented in Secs. 2.1 and 2.2 respectively. Both Sections follow a parallel structure: after introducing the classical PDF or the elements of the density matrix, the moments are defined as their multipolar expansion coefficients. Sec. 2.3 tackles with the properties of the moments for systems with special symmetry. The moments physical interpretation is presented in Sec. 2.4. Finally, the relation between the classical and the quantum mechanical moments will be discussed in Sec. 2.5 by introducing the "quantum population distribution function" 39, 40]. It represents the closer the quantum mechanics can get to the probability of finding $\mathbf{j}$ along a certain direction in the space and, in the correspondence limit, coincides with the classical PDF.

\subsection{Probability density function and classical polarisation moments}

\subsubsection{Probability density function (classical PDF)}

The classical description of $\mathbf{a} \mathbf{j}$ angular momentum polarisation is based on the probability density function $P\left(\theta_{j}, \phi_{j}\right)$ (classical PDF) whose values correspond to the probability of finding $\mathbf{j}$ pointing along the direction given by the polar angles $\theta_{j}$ and $\phi_{j}$.

From the mathematical point of view, $P\left(\theta_{j}, \phi_{j}\right)$ is just a function of the polar angles and, as such, it can be expanded in terms of standard spherical harmonics $Y_{k q}\left(\theta_{j}, \phi_{j}\right)$ [37] or, more conveniently [25, 41], in terms of complex conjugates of the $C_{k q}\left(\theta_{j}, \phi_{j}\right)$ modified spherical harmonics

$$
C_{k q}\left(\theta_{j}, \phi_{j}\right)=\left(\frac{4 \pi}{2 k+1}\right)^{1 / 2} Y_{k q}\left(\theta_{j}, \phi_{j}\right)
$$

The concrete expression for the expansion is given by 25

$$
P\left(\theta_{j}, \phi_{j}\right)=\sum_{k=0}^{\infty} \sum_{q=-k}^{k} \frac{(2 k+1)}{4 \pi} a_{q}^{(k)} C_{k q}^{*}\left(\theta_{j}, \phi_{j}\right)
$$

where the $a_{q}^{(k)}$ complex coefficients are the classical polarisation moments.

The PDF integral over the whole space, that represents the probability of finding $\mathbf{j}$ pointing along any spatial direction, can be interpreted as the population of the system whose angular momentum is $\mathbf{j}$. 


\subsubsection{Classical polarisation moments}

The orthogonality of the spherical harmonics 37.

$$
\int_{-1}^{1} \int_{0}^{2 \pi} C_{k^{\prime} q^{\prime}}^{*}\left(\theta_{j}, \phi_{j}\right) C_{k q}\left(\theta_{j}, \phi_{j}\right) d \cos \theta_{j} d \phi_{j}=\frac{4 \pi}{2 k+1} \delta_{k k^{\prime}} \delta_{q q^{\prime}}
$$

makes possible to find an explicit expression for the classical polarisation moments by inverting Eq. (2.2):

$$
a_{q}^{(k)}=\int_{-1}^{1} \int_{0}^{2 \pi} P\left(\theta_{j}, \phi_{j}\right) C_{k q}\left(\theta_{j}, \phi_{j}\right) d \cos \theta_{j} d \phi_{j}=\left\langle C_{k q}\left(\theta_{j}, \phi_{j}\right)\right\rangle
$$

which shows that each $a_{q}^{(k)}$ can be individually interpreted as the average of the corresponding modified spherical harmonic over the PDF angular distribution.

The $P\left(\theta_{j}, \phi_{j}\right)$ distribution values must be real. In other words, this means that the classical PDF must coincide with its complex conjugate. By imposing explicitly this condition it is found that the classical polarisation moments fulfill the following relation

$$
a_{q}^{(k)}=(-1)^{q} a_{-q}^{(k) *}
$$

\subsection{Density matrix and quantum mechanical polarisation moments}

The limitations coming from the uncertainty principle make impossible to talk about an angular momentum pointing along a defined spatial directions and to define a PDF with the same meaning as in the classical case. Because of this, to deal with the $\mathbf{j}$ polarisation in the frame of quantum mechanics it will be necessary to follow a different strategy.

The starting point for the quantum mechanical treatment will be the density operator $(\hat{\rho})$ that represents the state of the physical system under study. This operator will play the role of the classical PDF. It contains all the information about the system and, in particular, that corresponding to the $\mathbf{j}$ polarisation. After presenting its general features in Sec. 2.2.1, the quantum mechanical polarisation moments will be introduced in Sec. 2.2 .2 as the coefficients of the $\hat{\rho}$ matrix elements multipolar expansion. 


\subsubsection{Density operator and density matrix}

\section{Pure states}

The set of observables $\hat{A}, \hat{B}, \ldots, \hat{L}$ is called a "complete set of commuting observables" (CSCO) if it possesses an unique common basis system [34]. Given another observable $\hat{Q}$ which commutes with all the operators of the set, it will have the same basis and its eigenvalues $q$ will be a well defined function of the $a, b, \ldots, l$ eigenvalues of $\hat{A}, \hat{B}, \ldots, \hat{L}$. If $\hat{Q}$ does not commute with all the observables of the set, the measurement of its eigenvalues will not be compatible with the simultaneous knowledge of $a, b, \ldots, l$.

An experiment where the simultaneous measurement of the eigenvalues corresponding to a complete commuting set of observables is performed will be termed a "complete experiment" 26]. After that measurement, the dynamical state of the system will be given by the $|a, b, \ldots, l\rangle$ eigenstate of the $\hat{A}, \hat{B}, \ldots, \hat{L}$ observables included in the set.

Any system prepared in this way is said to be in a "pure" state and it has well defined dynamical properties. They are maximum information states (in the sense that it is possible to find a complete experiment which gives an unique result predictable with certainty when carried out over the system) and can be represented by a single state vector $|\phi\rangle$.

\section{Mixed states. The density operator}

In practise, a complete preparation of a system is only seldom achieved and the observables measured during the preparation process use not to be a complete set. When this happens the system is not in a pure state as it is not possible to find a complete experiment that, when performed, provides with an uniquely predetermined outcome.

These states have been called "mixed states" because they can be represented by an statistical mixture (an incoherent mixture) [26] of different pure states. In this case a single state vector can not describe the system (if it were enough it would be a pure state) but it is necessary to specify the probabilities $p_{1}, p_{2}, \ldots, p_{n}$ of finding the system in each of the $|1\rangle,|2\rangle, \ldots,|n\rangle$ pure states included in the mixture.

That statistical mixture can be completely characterised by means of an Hermitean operator called density operator and defined as

$$
\hat{\rho}=\sum_{r} p_{r}|r\rangle\langle r|
$$


where the $|r\rangle$ kets are normalised but they do not need to be orthogonal. The $p_{r}$ statistical weights are positive real numbers whose sum is the population of the state. If only one of these statistical weights were different from zero the system would be in a pure state and the density operator would be given by $\hat{\rho}=|\phi\rangle\langle\phi|$.

\section{Density matrix}

The density matrix $(\rho)$ is the representation of the density operator in an orthonormal basis $\left(\left|\phi_{n}\right\rangle\right)$ of the Hilbert space corresponding to the studied system. To obtain an expression for its elements we only have to expand the pure states included in the statistical mixture in terms of the basis vectors

$$
|r\rangle=\sum_{n} c_{r n}\left|\phi_{n}\right\rangle \quad\langle r|=\sum_{n^{\prime}} c_{r n^{\prime}}^{*}\left\langle\phi_{n^{\prime}}\right|
$$

and to substitute these expressions into the equation 2.6

$$
\hat{\rho}=\sum_{n, n^{\prime}}\left(\sum_{r} p_{r} c_{r n} c_{r n^{\prime}}^{*}\right)\left|\phi_{n}\right\rangle\left\langle\phi_{n^{\prime}}\left|=\sum_{n, n^{\prime}} \rho_{n, n^{\prime}}\right| \phi_{n}\right\rangle\left\langle\phi_{n^{\prime}}\right|
$$

The numbers

$$
\rho_{n, n^{\prime}}=\left\langle\phi_{n}|\hat{\rho}| \phi_{n^{\prime}}\right\rangle=\sum_{r} p_{r} c_{r n} c_{r n^{\prime}}^{*}
$$

are the elements of the density matrix in the $\left|\phi_{n}\right\rangle$ basis.

The following properties [42, 43] of the density matrix are necessary for the future development of theory as they will be repeatedly employed:

- The density matrix is Hermitian.

- The trace of the density matrix is equal to the population of the system

$$
\operatorname{tr}(\rho)=\sum_{r} p_{r}
$$

- Any diagonal element $\rho_{n, n}$ of the density matrix in any representation is nonnegative and its value represents the probability of finding the system in the $\left|\phi_{n}\right\rangle$ state.

- The off-diagonal elements of the density matrix express the coherence between the $\left|\phi_{n}\right\rangle$ states. There are three possibilities: 
1. All the density matrix elements are zero except one placed on the diagonal. This means that the system state can be described by the basis element corresponding to the non-zero diagonal element or, in other words, that the system is in the pure state corresponding to that ket.

2. All the off-diagonal elements are zero and several (more than one) diagonal elements are different from zero. In this case the system state is an incoherent superposition of the $\left|\phi_{n}\right\rangle$ basis states.

3. Two or more off-diagonal elements are different from zero. In this case the state of the system is a coherent superposition of the basis kets.

Pure states are a coherent superposition of the elements of any possible basis (in this sense, the pure states can be considered as fully coherent states). To prove this, let us suppose a system whose density operator is given by $\hat{\rho}=|\phi\rangle\langle\phi|$. By expanding $|\phi\rangle$ in an arbitrary basis $\left|\phi_{n}\right\rangle$

$$
|\phi\rangle=\sum_{n} c_{n}\left|\phi_{n}\right\rangle \quad\langle\phi|=\sum_{n^{\prime}} c_{n^{\prime}}^{*}\left\langle\phi_{n^{\prime}}\right|
$$

it is found that the density operator can be expressed as

$$
\hat{\rho}=\sum_{n, n^{\prime}}\left(c_{n} c_{n^{\prime}}^{*}\right)\left|\phi_{n}\right\rangle\left\langle\phi_{n^{\prime}}\left|=\sum_{n, n^{\prime}} \rho_{n, n^{\prime}}\right| \phi_{n}\right\rangle\left\langle\phi_{n^{\prime}}\right|
$$

where the elements of the density matrix have now a simple expression

$$
\rho_{n, n^{\prime}}=c_{n} c_{n^{\prime}}^{*}
$$

The ket $|\phi\rangle$ that represents the system state can coincide or not with one of the elements of the basis. If it coincides, the situation is trivial because we are in case 1 (the density matrix is diagonalized by the $\left|\phi_{n}\right\rangle$ basis and only one element of the diagonal is different from zero). On the contrary, if $|\phi\rangle$ does not belong to the basis, at least two off-diagonal elements have to be different from zero and the state of the system will be a coherent superposition of the $\left|\phi_{n}\right\rangle$ basis elements (case 3).

For physical systems which are not in a pure state, the coherent/incoherent character will depend on the chosen basis, that is, the state corresponding to the density operator (2.6) can be a coherent superposition of the elements of a basis and incoherent superposition of the elements of other basis. To clarify this let us suppose for a moment that the $|r\rangle$ kets in (2.6) are part of a basis (that 
is, they are orthonormal) and that more than one takes part in the sum. The system state would be an incoherent supperposition of the states of this basis because the $\hat{\rho}$ operator representation is diagonal with more than one non-zero diagonal elements (case 2):

$$
(\rho)=\left(\begin{array}{ccccccc}
p_{1} & & & & \vdots & & \\
& p_{2} & & \ldots & 0 & \ldots & \\
& & \ddots & & \vdots & & \\
& & & p_{r} & & & \\
& & & & 0 & & \\
& & \vdots & & & 0 & \\
& \ldots & 0 & \ldots & & & 0 \\
& & \vdots & & & &
\end{array}\right)
$$

However, if the $|r\rangle$ kets are expressed in another basis, it can happens that the corresponding representation of $\hat{\rho}$ displays non-zero off-diagonal elements (case 3) and the system state would be a coherent superposition of the elements of this second basis.

- Given a certain density matrix, it is not necessary to diagonalize it to decide wether it corresponds (only one eigenvalue different from zero) or not (more than one eigenvalue different from 0 ) to a pure state. It is enough to evaluate the trace of the density matrix square and to compare it to the population:

- When the system is in a pure state $\hat{\rho}$ turns out to be a projection operator $\left(\hat{\rho}=\hat{\rho}^{2}=\hat{\rho}^{\dagger}\right)$ and

$$
\operatorname{tr}\left(\rho^{2}\right)=\operatorname{tr}(\rho)
$$

- On the contrary, if

$$
\operatorname{tr}\left(\rho^{2}\right)<\operatorname{tr}(\rho)
$$

the system state will be a mixed one.

- While the maximum value of $\operatorname{tr}\left(\rho^{2}\right)$ is given by Eq. (2.15), the minimum is $\operatorname{tr}(\rho)^{2} / n$ (where $n$ is the dimension of the Hilbert space, that is, the number of basis elements). This represents the furthest than the physical system can be from a pure state (or the maximum degree of "mixture") and it corresponds to a density matrix which, when diagonalized, has all the eigenvalues equal and different from zero. 
The value of $\operatorname{tr}\left(\rho^{2}\right)$ is not altered by changes of basis, in such a way that a pure (mixed) state will be a pure (mixed) state in any basis (the fact of having or not a pure state is a property of the system and, as such, it can not depend on the basis selected for its analysis).

- The expectation value of any observable $\hat{A}$ is given by the trace of its product with the density operator $\hat{\rho}$

$\langle\hat{A}\rangle=\sum_{r} p_{r}\langle r|\hat{A}| r\rangle=\sum_{n, n^{\prime}}\left\langle\phi_{n}|\hat{\rho}| \phi_{n^{\prime}}\right\rangle\left\langle\phi_{n^{\prime}}|\hat{A}| \phi_{n}\right\rangle=\sum_{n, n^{\prime}} \rho_{n, n^{\prime}} A_{n^{\prime}, n}=\operatorname{tr}(\rho A)$

(of course, the value of $\langle\hat{A}\rangle$ is independent of the basis $\left(\left|\phi_{n}\right\rangle\right)$ choice). This expression can be found as well in this alternative form

$$
\langle\hat{A}\rangle=\sum_{r} p_{r}\langle r|\hat{A}| r\rangle=\frac{\operatorname{tr}(\rho A)}{\operatorname{tr}(\rho)}
$$

differing from Eq. (2.17) in the normalisation factor $\operatorname{tr}(\rho)$ (the system population). Throughout this work, Eq. (2.17) will be preferred to its normalised counterpart (2.18) whenever it will be necessary to evaluate expectation values from the density operator. This is just a matter of convention and, as such, it has advantages (the polarisation moments definition (Sec. 2.2.2) coincides with the one employed in [33] and in most of the theoretical works about stereodynamics) and disadvantages (the information necessary to determine the system population is included in the density operator and, therefore, the expectation values determined from (2.17) include that information and will depend on the population. Prior to compare expectation values corresponding to systems with different population, they have to be normalised dividing by the population). This discussion holds the same for the averaged values introduced in Sec. 2.1 (in particular, in Eq. (2.4) we have not divided by $\langle 1\rangle$, the classical counterpart of the quantum mechanical population). As it will be indicated in the following Section, the choice of Eq. (2.17) and the (eventual) necessity of normalising the expectation values will have consequences on the polarisation moments.

- In the Schrödinger picture of the quantum mechanics the temporal evolution of the states is determined by the expression

$$
|r ; t\rangle=\hat{U}\left(t, t_{0}\right)\left|r ; t_{0}\right\rangle
$$

with $\hat{U}\left(t, t_{0}\right)$ representing the time evolution operator and $|r ; t\rangle\left(\left|r ; t_{0}\right\rangle\right)$ a generic 
state at time $t\left(t_{0}\right)$.

The time evolution of the density operator (2.6) can be determined by application of the former equation to each of the states involved in the mixture

$$
\begin{aligned}
\hat{\rho}(t) & =\sum_{r} p_{r}|r ; t\rangle\langle r ; t| \\
& =\hat{U}\left(t, t_{0}\right)\left(\sum_{r} p_{r}\left|r ; t_{0}\right\rangle\left\langle r ; t_{0}\right|\right) \hat{U}^{\dagger}\left(t, t_{0}\right) \\
& =\hat{U}\left(t, t_{0}\right) \hat{\rho}\left(t_{0}\right) \hat{U}^{\dagger}\left(t, t_{0}\right)
\end{aligned}
$$

Taking now the first derivative of this expression with respect to time and substituting

$$
i \hbar \frac{\partial \hat{U}\left(t, t_{0}\right)}{\partial t}=\hat{H} \hat{U}\left(t, t_{0}\right)
$$

(and the corresponding one for the adjoint), we obtain the Liouville equation

$$
i \hbar \frac{\partial \hat{\rho}(t)}{\partial t}=[\hat{H}, \hat{\rho}(t)]
$$

that can be employed to obtain $\hat{\rho}(t)$.

- An explicit expression for $\hat{\rho}^{2}(t)$ can be derived from 2.20 by using the unitary character of the time evolution operator

$$
\hat{\rho}^{2}(t)=\hat{U}\left(t, t_{0}\right) \hat{\rho}^{2}\left(t_{0}\right) \hat{U}^{\dagger}\left(t, t_{\circ}\right)
$$

This involves that the trace of $\hat{\rho}^{2}(t)$ does not change with time or, in other words, that if a system is in a pure state it can not evolve into a mixture and vice versa (if $\hat{\rho}^{2}\left(t_{0}\right)=\hat{\rho}\left(t_{0}\right)$ at $t_{0}$ then $\hat{\rho}^{2}(t)=\hat{\rho}(t)$ at any time). The unitary nature of the evolution operator ensures as well that the trace of $\hat{\rho}(t)$ will not change with time (Eq,2.20).

\subsubsection{Quantum mechanical polarisation moments}

\section{Polarisation operators}

The quantum mechanical treatment of angular momentum polarisation is based on the polarisation operators $[26,28,33,36,37,38$. These operators play the role of the spherical harmonics employed in the classical treatment and can be defined in several alternative ways (for a summary see the App. D of reference [36]) which only differ 
by the normalisation and the phase. We will employ here

$$
\hat{T}(j)_{k q}=\sum_{m, m^{\prime}=-j}^{j}(-1)^{j-m}\left\langle j m^{\prime} j-m \mid k q\right\rangle\left|j m^{\prime}\right\rangle\langle j m|
$$

which corresponds to references [33] and [38] and assumes that our physical system is characterised by a single value of the $j$ quantum number. The kets $|j, m\rangle$ are the orthonormal eigenvectors of the square angular momentum operator $\left(\hat{\jmath}^{2}\right)$ and of the operator representing the angular momentum projection along a certain axis $z\left(\hat{\jmath}_{z}\right)$

$$
\begin{gathered}
\hat{\jmath}^{2}|j, m\rangle=\hbar^{2} j(j+1)|j, m\rangle \\
\hat{\jmath}_{z}|j, m\rangle=\hbar m|j, m\rangle \quad m=-j, \ldots, j
\end{gathered}
$$

(if the $\hat{\jmath}^{2}$ and $\hat{\jmath}_{z}$ operators were not a complete commuting set, the $|j, m\rangle$ kets must be substituted by $|\tau, j, m\rangle$ (with $\tau$ representing the quantum numbers necessary to fully specify the state of the system). In this case, the following discussion would hold for physical systems with univocally defined values of $\tau$ and $j$ ). By means of $\left\langle j m^{\prime} j-m \mid k q\right\rangle$ is represented a Clebsch-Gordan coefficient [37. Their symmetry properties limit the number of possible $\hat{T}(j)_{k q}$ operators to those fulfilling

$$
k=0, \ldots, 2 j \text { and } q=-k, \ldots, k
$$

as otherwise the Clebsch-Gordan coefficients vanish.

Given two reference frames $x y z$ and $X Y Z$, the polarisation operators defined in each system will be related to each other through the expression

$$
\hat{T}(j)_{k q}=\sum_{Q=-k}^{k} \hat{T}(j)_{k Q} D_{q Q}^{(k) *}(\alpha, \beta, \gamma)
$$

where the indices $q(Q)$ refer to the $x y z(X Y Z)$ frame, $D^{(k)}(\alpha, \beta, \gamma)$ is a Wigner rotation matrix [37] and $(\alpha, \beta, \gamma)$ are the Euler angles associated with the rotations that take the $x y z$ frame into the $X Y Z$ frame (see App. A for a derivation of the equation and a brief summary of the concepts and conventions underlying).

Any set of $(2 k+1)$ operators that transform under rotations according to Eq. (2.28) is said to form an spherical tensor operator of rank $k$. Their matrix elements can be evaluated by using the Wigner-Eckart theorem [37, 38, that, when applied to 
polarisation operators, allows for writing

$$
\left\langle j m^{\prime}\left|\hat{T}(j)_{k q}\right| j m\right\rangle=(-1)^{2 k}\left\langle j m k q \mid j m^{\prime}\right\rangle \frac{\left\langle j|| \hat{T}(j)_{k} \| j\right\rangle}{\sqrt{2 j+1}}
$$

where the Clebsch-Gordan coefficient represents a geometrical factor that contains all the information about the coordinate system orientation (through the quantum numbers $m, m^{\prime}$ and $q$ ) and the $\left\langle j\left\|\hat{T}(j)_{k}\right\| j\right\rangle$ factor is called the "reduced matrix element" and concentrates the dynamical (non geometrical) information. Comparing (2.29) with the value of $\left\langle j m^{\prime}\left|\hat{T}(j)_{k q}\right| j m\right\rangle$ that could be directly obtained from Eq. (2.24) it is possible to obtain an expression for the reduced matrix element

$$
\left\langle j|| \hat{T}(j)_{k} \| j\right\rangle=(2 k+1)^{1 / 2}
$$

which, for every k-rank tensor, is simply a constant. This involves that the polarisation operators are purely geometrical operators and that their matrix elements are purely geometrical quantities.

The $\hat{T}(j)_{k q}$ operator and its adjoint $\hat{T}^{\dagger}(j)_{k q}$ are connected by

$$
\hat{T}^{\dagger}(j)_{k q}=(-1)^{q} \hat{T}(j)_{k-q}
$$

This equality makes possible to prove an useful relation between the traces of the matrix representations (in the $|j, m\rangle$ basis) of the polarisation operators and their adjoints

$$
\operatorname{tr}\left(T(j)_{k q} T^{\dagger}(j)_{k^{\prime} q^{\prime}}\right)=\delta_{k k^{\prime}} \delta_{q q^{\prime}}
$$

Fixing on $k^{\prime}=q^{\prime}=0$ in this equation and taking into account that

$$
\hat{T}(j)_{00}=\hat{T}^{\dagger}(j)_{00}=\frac{1}{(2 j+1)^{1 / 2}} \hat{1}
$$

it is found the following expression for the trace of a single polarisation operator

$$
\operatorname{tr}\left(T(j)_{k q}\right)=(2 j+1)^{1 / 2} \delta_{k 0} \delta_{q 0}
$$

that turns out to be different from 0 only when $k$ and $q$ are equal to 0.

The necessity for this detailed presentation of the $\hat{T}(j)_{k q}$ operators and their properties will become clear in next pages, where it will be showed that they serve as a basis for the multipolar expansion of the density operator and that the quantum polarisation moments can be expressed as the $\left\langle\hat{T}^{\dagger}(j)_{k-q}\right\rangle$ expectation values. 


\section{Quantum polarisation moments}

The density operator $\hat{\rho}$ that represents the state of the system can be written ${ }^{2}$ as

$$
\hat{\rho}=\sum_{m, m^{\prime}}\left\langle j m^{\prime}|\hat{\rho}| j m\right\rangle\left|j m^{\prime}\right\rangle\langle j m|
$$

The orthogonality of the Clebsch-Gordan coefficients [37] allows for turning around Eq. (2.24)

$$
\left|j m^{\prime}\right\rangle\langle j m|=\sum_{k, q}(-1)^{j-m}\left\langle j m^{\prime} j-m \mid k q\right\rangle \hat{T}(j)_{k q}
$$

and, substituting this expression into Eq. (2.35), $\hat{\rho}$ can be expressed in terms of the polarisation operators

$$
\hat{\rho}=\sum_{k, q}\left(\sum_{m, m^{\prime}}(-1)^{j-m}\left\langle j m^{\prime}|\hat{\rho}| j m\right\rangle\left\langle j m^{\prime} j-m \mid k q\right\rangle\right) \hat{T}(j)_{k q}
$$

By multiplying both sides of this equation by $\hat{T}^{\dagger}(j)_{k^{\prime} q^{\prime}}$, taking the trace and using Eq. (2.17) and (2.32), it can be seen that the content of the parenthesis is anything but $\left\langle\hat{T}^{\dagger}(j)_{k q}\right\rangle$. In consequence, the density operator can be expanded as

$$
\hat{\rho}=\sum_{k=0}^{2 j} \sum_{q=-k}^{k}\left\langle\hat{T}^{\dagger}(j)_{k q}\right\rangle \hat{T}(j)_{k q}
$$

and its matrix elements will be given by

$$
\left\langle j m^{\prime}|\hat{\rho}| j m\right\rangle=\sum_{k=0}^{2 j} \sum_{q=-k}^{k}(-1)^{j-m}\left\langle j m^{\prime} j-m \mid k q\right\rangle\left\langle\hat{T}^{\dagger}(j)_{k q}\right\rangle
$$

The complex expansion coefficients $\left\langle\hat{T}^{\dagger}(j)_{k q}\right\rangle$ are called "State multipoles" or "statistical tensor" [33, 44, 45]. They constitute a complete description of the system state, entirely analogous to that given by the $\hat{\rho}$ matrix elements.

The Hermitean character of the density operator (in Eq. (2.38) ) and of its matrix representation (in Eq. (2.39)) involves the following relation between the $q$ and $-q$ state multipoles

$$
\left\langle\hat{T}^{\dagger}(j)_{k q}\right\rangle=(-1)^{q}\left\langle\hat{T}^{\dagger}(j)_{k-q}\right\rangle^{*}
$$

The quantum mechanical polarisation moments will be defined in terms of the state

\footnotetext{
${ }^{2}$ Employing the $|j m\rangle$ basis completeness
} 
multipoles as

$$
a_{q}^{(k)}=(-1)^{q}\left(\frac{2 j+1}{2 k+1}\right)^{1 / 2}\left\langle\hat{T}^{\dagger}(j)_{k-q}\right\rangle
$$

being the complex coefficients of the following expansion of the density matrix elements

$$
\left\langle j m^{\prime}|\hat{\rho}| j m\right\rangle=\sum_{k=0}^{2 j} \sum_{q=-k}^{k} \frac{2 k+1}{2 j+1} a_{q}^{(k)}\left\langle j m^{\prime} k q \mid j m\right\rangle
$$

As before, the Clebsch-Gordan orthogonality can be used to invert this equation obtaining

$$
a_{q}^{(k)}=\sum_{m, m^{\prime}=-j}^{j}\left\langle j m^{\prime}|\hat{\rho}| j m\right\rangle\left\langle j m^{\prime} k q \mid j m\right\rangle
$$

which, given any density matrix, provides with the corresponding polarisation moments for the system.

Combining the definition $(2.41)$ with the relation $(2.40)$ gives

$$
a_{q}^{(k)}=(-1)^{q} a_{-q}^{(k) *}
$$

which is a direct consequence of the $\hat{\rho}$ operator hermiticity. This expression is identical to Eq. (2.5) (found in the classical case and derived using the real character of the classical PDF function).

\subsection{Polarisation moments for special systems}

In this Section, special properties of the classical and quantum mechanical polarisation moments for systems with axial or reflection symmetry and for unpolarised systems are obtained.

The two former situations will be important for the later discussion of the atomdiatom collisions mechanism, as they can display both symmetries. The existence of an axis or a plane of symmetry will constrain the values that the polarisation moments can take and, as it is proved now, the mathematical expression for these restrictions can be simply deduced from the equality

$$
a_{q}^{(k)}=\sum_{Q=-k}^{k} A_{Q}^{(k)} D_{q Q}^{(k) *}(\alpha, \beta, \gamma)
$$


that describes how the moments behave under rotation and holds regardless of their classical or quantum mechanical origin (see Apendix $\underline{\mathrm{A}}$ ). In this equation, $a_{q}^{(k)}$ and $A_{Q}^{(k)}$ correspond to the polarisation moments of our physical system referred to the $x y z$ and $X Y Z$ frames respectively, $(\alpha, \beta, \gamma)$ are the Euler angles which take $x y z$ into $X Y Z$ and $D_{q Q}^{(k) *}(\alpha, \beta, \gamma)$ represents a rotation matrix [37.

The Section finishes with a discussion of unpolarised systems, that is, systems whose angular momentum is randomly distributed. It will be proved that the $k=0$ moment suffices to describe the corresponding $\mathbf{j}$ distribution.

\subsubsection{Axially symmetric systems}

Let us suppose that our system is axially symmetric with respect to the $z$ axis. This implies that the properties of the system are the same regardless the choice of the $x$ and $y$ axis perpendicular to $z$ or, alternatively, that the properties of the system are unchanged as long as they are considered in frames which differ only by a rotation about the $z$ axis. In the context of the classical mechanics, this means that the PDF and the classical polarisation moments (univocally related through the Eq. (2.2)) must be identical in all those frames. From the quantum mechanical point of view the reasoning is slightly more subtle although it takes us to the same conclusion. By virtue of the axial symmetry any measurement of an observable must be independent on the $x$ and $y$ definition. The polarisation moments are not observables (because they are complex numbers defined as the expectation value of a non-hermitian operator) but, as it will be shown in next Section and in App. B, their real and imaginary parts do are. This means that, if the system displays axial symmetry, the quantum mechanical moments will be identical in frames connected by means of a rotation about the $z$ axis.

Such as a rotation can be represented through the $(\alpha, 0,0)$ set of Euler angles. Mathematically, the connection between the moments referred to the $x y z$ and $X Y Z$ frames (related through that $z$ axis rotation) is given by Eq. (2.45)

$$
a_{q}^{(k)}=\sum_{Q=-k}^{k} A_{Q}^{(k)} \underbrace{D_{q Q}^{(k) *}(\alpha, 0,0,)}_{\delta_{q Q} e^{i q \alpha}}=A_{q}^{(k)} e^{i q \alpha} \quad \forall k, q, \alpha
$$

Simultaneously, the axial symmetry implies that the moments in both frames are the same

$$
a_{q}^{(k)}=A_{q}^{(k)} \quad \forall k, q
$$


Conditions 2.46 and 2.47 only can be simultaneously fulfilled if

$$
a_{q}^{(k)}=0 \quad q \neq 0
$$

So, the axially symmetric systems are characterised by $q=0$ moments as those with $q \neq 0$ are necessarily null.

Next Section will furnish a directional interpretation of the moments. In particular, it is stated that $k$ even (odd) moments are related to the alignment (orientation) of the angular momentum. From this point of view, what Eq. (2.48) indicates is that an axially symmetric system can be aligned and also oriented.

\subsubsection{Systems with a plane of symmetry}

Let us next suppose that our system is invariant under reflections through a plane that, for reasons that will become clear in next Chapters, will be identify with the $x z$ one.

The discussion of Eq. (2.45) and the analysis of systems with axial symmetry have been presented by assuming that it was the frame of reference who displayed the consequences of the symmetry transformation while the physical system remained unchanged. However, this is not the only possibility and it could have been studied from other point of view: the transformation is undergone by the physical system while the frame of reference does not change. To choose one or the other is just a matter of convention as both of them take to identical results (although, once that a convention is adopted, it is necessary to be consequent with the election).

The effect that a plane of symmetry has on the polarisation moments can be determined by using the fact that the system properties must be the same in frames of reference related through a reflection about that plane. However, this way of working is not convenient now because reflections of the frame invert its chirality (take a right handed frame into a left one and vice versa), complicating the analysis. This difficulty does not arise if the second of the points of view formerly exposed is considered, that is, the reflection is assumed to transform the physical system and not the frame of reference. Therefore, this is the convention that will be employed now.

In this context, the existence of a plane of symmetry involves that the physical properties of the system must not be altered by its reflection through that plane. This means that, in particular, the polarisation moments will remain unchanged as neither the PDF (classical mechanics) nor the observables (quantum mechanics) change under reflection.

Combination of this invariance with the expression that relates the moments for 
the original and the reflected system will allow for establishing how the values of the moments are conditioned by the reflection symmetry. To work out that expression it is convenient to remember that the effect of a reflection through the $x z$ plane is equivalent to the effect of the parity (coordinate inversion) followed by a $\pi$ radians rotation about the $y$ axis [46]. Let us examine separately the effect of the two operations.

The parity has no effect on the polarisation moments. Classically, this can be understood by considering the axial nature of the angular momentum vector [47]. This means that its components do not change under parity (the position vectors and the linear momentums invert simultaneously their sense, leaving the angular momentum unchanged) and, in consequence, the classical moments are not altered. Quantum mechanically, the effect of the parity operator on the $|j, m\rangle$ basis vectors consist of multiplying them by an $(-1)^{j}$ phase factor [34] and, as long as the system under consideration is characterised by an integer value of $j$ (this will always be the case in the present work), this phase will not modify the $\left\langle j m|\hat{\rho}| j m^{\prime}\right\rangle$ matrix elements. As these elements are related to the polarisation moments through Eq. (2.42), these will be unchanged too.

Therefore, the relation between the moments of the initial physical system and the reflected one is exactly the same that exists between the moments of the initial system and a second one obtained by rotating it $\pi$ radians about the $y$ axis. This relation can be obtained from Eq. (2.45) with the proviso that its elements should be interpreted in a way consistent with the adopted convention (effects of the transformation undergone by the physical system). The proper interpretation in this case is the following: if the $a_{q}^{(k)}$ set represents the moments of a system that undergoes a rotation described by the $(-\gamma,-\beta,-\alpha)$ Euler angles ${ }^{3}$, the new moments of the system will be given by the $A_{Q}^{(k)}$ quantities in Eq. (2.45) (watch out with the change of sign and order of the angles), where both sets of moments are referred to the same $x y z$ frame.

As a $\pi$ radians rotation of the system about the $y$ axis corresponds to the $(0, \pi, 0)$ Euler angles, the moments of the system before and after the reflection are related by

$$
a_{q}^{(k)}=\sum_{Q=-k}^{k} A_{Q}^{(k)} \underbrace{D_{q Q}^{(k) *}(0,-\pi, 0)}_{(-1)^{k+q} \delta_{q-Q}}=(-1)^{k+q} A_{-q}^{(k)} \quad \forall k, q
$$

Beside, as the system is invariant under this reflection, the polarisation moments must

\footnotetext{
${ }^{3} \mathrm{~A}$ rotation of the frame of reference given by the $(\alpha, \beta, \gamma)$ set of Euler angles is equivalent to a rotation of the physical system given by $(-\gamma,-\beta,-\alpha)$. See reference 48 for more details
} 
fulfill simultaneously the condition (2.47). Both equations can be combined to give

$$
a_{q}^{(k)}=(-1)^{k+q} a_{-q}^{(k)} \quad \forall k, q
$$

for the moments of a system with reflection symmetry about the $x z$ plane.

It was proved in Secs. 2.1 and 2.2 that the polarisation moments must always satisfy the condition

$$
a_{q}^{(k)}=(-1)^{q} a_{-q}^{(k) *} \quad \forall k, q
$$

that, when merged with Eq. (2.50), provides with the following expression

$$
a_{q}^{(k)}=(-1)^{k+q} a_{-q}^{(k)}=(-1)^{k} a_{q}^{(k) *} \quad \forall k, q
$$

Two conclusions can be inferred from this constrain:

- $k$ odd (even) moments must be pure imaginary (real).

- The moments corresponding to odd values of $k$ and $q=0$ will vanish.

\subsubsection{Unpolarised systems}

A physical system is said to be unpolarised if its angular momentum is isotropically distributed.

Classically, this means that the probability of finding the $\mathbf{j}$ vector pointing along a defined direction in the space is the same for all the directions. Therefore, the classical PDF function does not depend on the polar angles and has an spherical shape. The only spherical harmonics that can contribute to the PDF in Eq. (2.2) is the $C_{00}\left(\theta_{j}, \phi_{j}\right)$, as any other contribution would distort that sphere, introducing a preference for a certain direction. In consequence, all the moments but that with $k=0$ must be null.

Quantum mechanically the conclusion is the same. The density operator corresponding to an unpolarised system (of population equal to one) is 33

$$
\hat{\rho}=\frac{1}{2 j+1} \sum_{m}|j m\rangle\langle j m|=\frac{1}{2 j+1} \hat{1}
$$

which reflects the non preference for any spatial direction (the Eq. (2.53) indicates that all the angular momentum projections over the $z$ axis are equally probable. As that density operator is unchanged under rotations ${ }^{4}$, this holds for any spatial direction

\footnotetext{
${ }^{4}$ The unitary character of the rotation operator $\hat{R}$ determines that, in this case, the rotated density operator $\hat{R} \hat{\rho} \hat{R}^{\dagger}$ is equal to $\hat{\rho}$
} 
and not only for the $z$ axis. So, the angular momentum is not oriented or aligned along any direction). The insertion of this density matrix (proportional to the unit matrix) elements into Eq. (2.43) and the usage of the Clebsch-Gordan coefficients properties allow for proving that, as in the classical case, only the $k=0$ moment can be different from 0 .

\subsection{Directional interpretation of the polarisation moments}

So far we have presented the polarisation moments as a set of complex numbers that represent an alternative description of the classical PDF or the density matrix, depending on whether classical or quantum mechanics is being used. In this Section it will be shown that their importance does not only lies on this fact but also on their role in the analysis of the system angular momentum $(\mathbf{j})$ spatial arrangement.

All the directional information about the angular momentum of a physical system is contained in the $a_{q}^{(k)}$ complex polarisation moments (each $k \neq 0$ moment represents a particular way of distorting (that is, polarising) an spherical (unpolarised) spatial distribution of the angular momentum (see 2.3.3)). However, to make this obvious they have to be combined in a suitable way such that the resulting moments describe qualitative and quantitatively the alignment or orientation of $\mathbf{j}$ along certain directions in the space. Those combinations, their properties and their interpretation will be the goal of this Section.

The complex polarisation moments can not be directly related to the Cartesian directions and, in order to have quantities with a defined directional meaning, they have to be mixed so that the resulting quantities are real: the real polarisation moments $\left(a_{q}^{\{k\}}\right)$. The sign of these new moments answers to questions like "is $\mathbf{j}$ alignment parallel or antiparallel to the $z$ axis?" or "what is the sense of the angular momentum orientation along the $x$ axis?".

Real moments are extremely useful when tackling the angular momentum polarisation study. However, they do not completely solve the problem because, on top of knowing the directional preferences of the angular momentum, it is essential to set how significative are those preferences. This information will be obtained by means of the renormalised polarisation moments. They answer questions like "what is the importance of the alignment or orientation related to each moment when compared to the unpolarised distribution?", "is that alignment/orientation influence on the $\mathbf{j}$ distribution important or is it insignificant?" or "which of two systems is more po- 
larised?".

The results included in this Section hold regardless the classical or quantum mechanical nature of the moments. A detailed mathematical discussion about their origin and justification can be found in App. B.

\subsubsection{Real polarisation moments}

The $a_{q}^{(k)}$ moments defined in Sec. 2.1 and 2.2 are complex coefficients which can not be associated to Cartesian directions [37. In order to have quantities directly related to the frame of coordinates, the complex moments have to be combined so that they form real moments $\left(a_{q}^{\{k\}}\right)$ tied to well-defined directions in the space. The relation between these sets of moments is given by $[49,50$.

$$
\begin{aligned}
a_{q+}^{\{k\}} & =\frac{1}{\sqrt{2}}\left[(-1)^{q} a_{q}^{(k)}+a_{-q}^{(k)}\right] \quad 1 \leq q \leq k \\
a_{0}^{\{k\}} & =a_{0}^{(k)} \\
a_{q-}^{\{k\}} & =\frac{1}{i \sqrt{2}}\left[(-1)^{q} a_{q}^{(k)}-a_{-q}^{(k)}\right] \quad 1 \leq q \leq k
\end{aligned}
$$

Incidentally, these are also the equations that relate real hydrogenic electronic orbitals such as $p_{x}$ and $p_{y}$ to the complex spherical harmonics $Y_{11 \pm}(\theta, \phi)$ or the $d_{x^{2}-y^{2}}$ and $d_{x y}$ to $Y_{22 \pm}(\theta, \phi)$. These expressions can be simplified by using Eq. (2.5) (or (2.44))

$$
\begin{aligned}
& a_{q+}^{\{k\}}=\sqrt{2}(-1)^{q} \operatorname{Re}\left[a_{q}^{(k)}\right] \quad 1 \leq q \leq k \\
& a_{0}^{\{k\}}=a_{0}^{(k)} \\
& a_{q-}^{\{k\}}=\sqrt{2}(-1)^{q} \operatorname{Im}\left[a_{q}^{(k)}\right] \quad 1 \leq q \leq k
\end{aligned}
$$

where Re and Im represent the real and the imaginary part of the corresponding complex moment.

The symmetry properties of the system can impose severe restrictions on the real moments that can be different from zero. For instance,

- All the real moments except those with $q=0$ will necessarily vanish for axially symmetric systems (Sec. 2.3.1).

- All the real moments except

$$
\begin{array}{ll}
a_{q+}^{\{k\}} & 1 \leq q \leq k \quad k \text { even } \\
a_{0}^{\{k\}} & k \text { even } \\
a_{q-}^{\{k\}} & 1 \leq q \leq k \quad k \text { odd }
\end{array}
$$




\begin{tabular}{|c|c|c|c|}
\hline \multirow[b]{2}{*}{$\mathrm{k}$} & \multirow[b]{2}{*}{$\mathrm{q}$} & \multicolumn{2}{|c|}{ Directional meaning } \\
\hline & & When positive & When negative \\
\hline 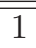 & 1 & orientation parallel to $y$ & בorientation antiparallel to $\hat{y}$ \\
\hline 1 & 0 & orientation parallel to $z$ & orientation antiparallel to $\hat{z}$ \\
\hline 1 & $1+$ & orientation parallel to $x$ & orientation antiparallel to $\hat{x}$ \\
\hline 2 & $2-$ & alignment along $x+y$ & alignment along $x-y$ \\
\hline 2 & $1-$ & alignment along $y+z$ & alignment along $y-z$ \\
\hline 2 & 0 & alignment along $z$ & alignment perpendicular to $z$ \\
\hline 2 & $1+$ & alignment along $x+z$ & alignment along $x-z$ \\
\hline 2 & $2+$ & alignment along $x$ & alignment along $y$ \\
\hline
\end{tabular}

Table 2.1.: Directional meaning of the real polarisation moments for $k=1$ and 2 .

will necessarily vanish for systems whose $x z$ plane is a plane of symmetry (Sec. 2.3.2).

\subsubsection{Meaning of the real polarisation moments}

Classically, the zero rank $(k=0)$ moment corresponds to the PDF average over all the directions in the space (See Eq. (2.4))

$$
a_{0}^{\{0\}}=a_{0}^{(0)}=\int_{-1}^{1} \int_{0}^{2 \pi} P\left(\theta_{j}, \phi_{j}\right) d \cos \theta_{j} d \phi_{j}
$$

and quantum mechanically it is the trace of the density matrix (Eq. (2.43))

$$
a_{0}^{\{0\}}=a_{0}^{(0)}=(2 j+1)^{1 / 2}\left\langle\hat{T}^{\dagger}(j)_{00}\right\rangle=\operatorname{tr}(\rho)
$$

In both cases, it can be considered as a normalization constant whose value coincides with the system population. As it will be explained in next Section, its value is related to the maximum and the minimum values than the $k \neq 0$ moments can take.

The $k \neq 0$ real moments with $\mathrm{k}$ odd (even) rank enlighten us about the sense of the orientation (alignment) of $\mathbf{j}$ with respect to certain directions [25, 33]. Their directional meaning is summarised in the Table 2.1 for $k=1$ and 2. For instance, if the $a_{1-}^{\{1\}}$ moment had a positive value that would mean that $\mathbf{j}$ is preferably oriented along the positive $y$ axis while if the value of the moment were negative the preferred orientation would be along the negative $y$ axis. In the same way, a positive (negative) value of the $a_{0}^{\{2\}}$ moment would mean that $\mathbf{j}$ is preferably aligned parallel (perpendicular) to the $z$ axis. The $a_{1+}^{\{2\}}$ moment contains information on the $\mathbf{j}$ alignment along the $x+z$ direction (positive values) or along the $x-z$ direction (negative values). 
The zero value corresponds to $\mathbf{j}$ contained in the $x y$ or $y z$ planes. The $a_{2+}^{\{2\}}$ moment gauges the relative preference for $\mathbf{j}$ alignment along the $x$ axis (positive values) with respect to the $y$ axis (negative values). The real moments corresponding to $k$ values larger than 2 are more difficult to visualise and, in consequence, the information they contain is harder to interpret.

It is now evident why for the unpolarised systems only the $k=0$ moment can be different from zero (see Sec. 2.3.3). As the angular momentum does not display alignment or orientation along any direction (because $\mathbf{j}$ does not show preferences for any direction), all the $k \neq 0$ real (and complex) moments have to be necessarily null.

It is important to point out that the complex and real moments are not, in essence, different as they contain the same information although presented in a different way (there is, however, a subtle difference between them when considered in the quantum mechanical context. In this case, real (complex) moments correspond to the expectation value of Hermitean (non Hermitean) operators and are (not) observable quantities (see App. B ) ). In practise, while the real moments are more appropriate for discussing directional preferences the complex moments are better for mathematical manipulations [36]. This is the reason why mathematical derivations will be performed with complex moments and numerical results and figures will be presented in terms of their real counterparts.

\subsubsection{Renormalised polarisation moments}

Employing Table 2.1 or others for higher rank values [50] it is possible to interpret the $(k \neq 0)$ real polarisation moments in terms of $\mathbf{j}$ directional preferences. Useful as this achievement is, it does not suffice to provide with a complete picture of the angular momentum polarisation because we still do not know how intense are those preferences.

Both the real and the complex moments contain information not only about the directions of $\mathbf{j}$ but also on the system population, that is, on the $a_{0}^{\{0\}}$ magnitude (see 25, 36] and the discussion about the expectation values in Sec. 2.2.1). This means that the same value of the $a_{q}^{\{k\}}$ (or $a_{q}^{(k)}$ ) moments for two systems with different population corresponds to a $\mathbf{j}$ alignment or orientation of different intensity in each case or, equivalently, that the range of accessible values for the $k \neq 0$ moments depends on the population (See App. B for an example). As a consequence, unless the value of the population is the same, the moments defined so far can not be directly used to compare the polarisations of two physical systems.

It is the same problem that would appear if we try to measure distances employing 
maps with different scales. The best way of overcoming this difficulty is to choose and scale, to draw all the maps to that scale and then to compare them. Equally, the best way of comparing polarisations of systems with different population is to adopt one value of the population and the corresponding scale and to refer all the systems polarizations to them. We will choose the scale corresponding to systems with population equal to one (it can be found in App. B for the quantum mechanical case). In practise, this means that whenever it will be necessary to set the intensity of a polarisation or to compare two different polarisations of the system we will employ the renormalised polarisation moments 25 .

$$
\frac{a_{q}^{\{k\}}}{a_{0}^{\{0\}}} \quad \forall k, q
$$

(where the equations holds the same for complex moments). These moments, together with the corresponding scale, make possible to answer all the questions posed in the introduction of the Section.

From the physical point of view, our choice is equivalent to use the unpolarised system as "polarisation intensity unit of measure" because when we divide every moment by the population we are measuring the importance of the polarisation with respect to the unpolarised system (which is completely defined by means of the population).

\subsection{Classical and quantum-mechanical moments comparison. Quantum PDF.}

Frequently, classical and quantum mechanical methods are simultaneously used to study the physical properties of a system (the reaction dynamics is an example of this duality). If this study includes the angular momentum polarisation, it will provide with two different sets of polarisation moments: a classical and a quantum mechanical one. On top of analysing them separately, it is convenient to compare their characteristics, as this comparison makes possible to distinguish the genuine quantum effects and it is useful to interpret the meaning of those quantum features which have a classical counterpart.

By defining the "quantum population distribution function" (quantum PDF) and by comparing it with the classical PDF it will be showed that the classical and the quantum mechanical moments can be directly compared. On top of enlightening about that comparison, the quantum PDF will allow for the rigorous and simple visualisation of the $\mathbf{j}$ distribution corresponding to each set of polarisation moments. 


\subsubsection{Quantum population distribution function}

The quantum PDF is given by [39, 40,

$$
Q\left(\theta_{j}, \phi_{j}\right)=\sum_{k=0}^{2 j} \sum_{q=-k}^{k} \frac{2 k+1}{4 \pi} a_{q}^{(k)}\langle j j k 0 \mid j j\rangle C_{k q}^{*}\left(\theta_{j}, \phi_{j}\right)
$$

where $a_{q}^{(k)}$ represent the quantum complex polarisation moments, $\langle j j k 0 \mid j j\rangle$ is a Clebsch-Gordan coefficient [37] and $C_{k q}^{*}\left(\theta_{j}, \phi_{j}\right)$ is the complex conjugate of a modified spherical harmonic. It describes the angular momentum polarisation in terms of the continuous spherical angles $\theta_{j}$ and $\phi_{j}$, constituting an exact quantum mechanical representation of the angular momentum distribution (that is, obtained with no recourse to the vector model or any other approximation, classical or otherwise). In contrast with the classical PDF (Eq. (2.2)), the values of this distribution do not represent the probability of finding $\mathbf{j}$ pointing along the direction specified by $\theta_{j}$ and $\phi_{j}$ but the population of the minimum uncertainty state (giving a certain direction, it is the state in which the angular momentum is as localised as possible around it. It coincides with the $|j j\rangle$ state, that is, the state in which the projection of $\mathbf{j}$ takes its maximum value along the considered direction) lying along the direction given by $\theta_{j}$ and $\phi_{j}$.

The only difference between the expressions for the quantum and the classical PDF is the appearance of a Clebsch-Gordan coefficient in the quantum mechanical formula (the difference in the upper limit of the sum over $k$ is just a consequence of its presence because $\langle j j k 0 \mid j j\rangle$ is null whenever $k>2 j$ ). As it was thoroughly discussed in reference [39, this coefficient (a real number whose absolute value is lower or equal than one) expresses the fact that the uncertainty principle places a limit on how polarised the angular momentum can be by changing the expansion functions from $C_{k q}^{*}\left(\theta_{j}, \phi_{j}\right)$ into $\langle j j, k 0 \mid j j\rangle C_{k q}^{*}\left(\theta_{j}, \phi_{j}\right)$. In the correspondence principle limit 34, where the uncertainty principle ceases to be significant, the quantum and the classical PDF become identical since [37, 39]

$$
\langle j j k 0 \mid j j\rangle \stackrel{j \rightarrow \infty}{\longrightarrow} 1
$$

The fact that the uncertainty principle limitations are taken into account by means of the Clebsch-Gordan coefficient makes clear the meaning of the polarisation moments: they contain all the angular momentum distribution symmetries and, dy- 
namica ${ }^{\sqrt{5}}$ effects apart, these symmetries do not depend on whether the uncertainty principle is being considered for their analysis. Therefore, classical and quantum mechanical moments can be directly compared without any "quantum correction" for the former [39, 40]. The dissimilarities between both sets of moments will be related to differences between the classical and the quantum dynamics of the system.

Although physically correct, comparing individually every classical and quantum mechanical moment can be an exhausting task and, beside, it does not use to provide with a global view of the importance and meaning of the symmetries contained in each set of moments.

The way of visualising those symmetries in a simple and direct way and of meaningfully performing the comparison is through the corresponding angular momentum distributions, that is, by comparing and analysing the shapes of the classical and the quantum mechanical PDFs. However, this comparison must be carefully done, taking into account that the differences between both PDFs can be divided into two different groups: (i) those coming from the dynamics (differences in the polarisation moments) and (ii) those coming from the consideration or not of the uncertainty principle. To avoid that the differences steaming from the second group "interfere" with those due to the former (whose analysis uses to be more interesting) is why the procedure proposed to perform the comparison [39, 40] does not employ the classical PDF but a quasiclassical PDF obtained by substituting the classical moments on the expression for the quantum PDF (it is quasiclassical because classical mechanics is used to determine the symmetries of the angular momentum distribution (Eq. (2.4) serves to evaluate the polarisation moments) but the shape associated with those symmetries is generated using the quantum mechanical expression Eq. (2.60) $)$.

The shape of the quasiclassical PDF takes into account the uncertainty principle by including the Clebsch-Gordan coefficient, with the subsequent limitation of the classical moments that contribute to those fulfilling $k \leq 2 j$ (instead of the $\infty$ moments that are necessary to determine the classical PDF (Eq. (2.2))). In consequence, any difference between this PDF and the quantum one will be due to differences between the classical and the quantum dynamical properties of the system (and never to the uncertainty principle) and their agreement will be as good (or as bad) as the agreement between the two sets of polarisation moments.

Fig 2.1 illustrates the properties of the quantum and the quasiclassical PDFs with an example corresponding to a pure $|j=2 m=2\rangle$ state. Quantum mechanically, this

\footnotetext{
${ }^{5}$ The molecules whose polarisation will be considered are not static but are taking part of a chemical reaction. In consequence, the dynamics of this process can influence on the polarisation moments values
} 

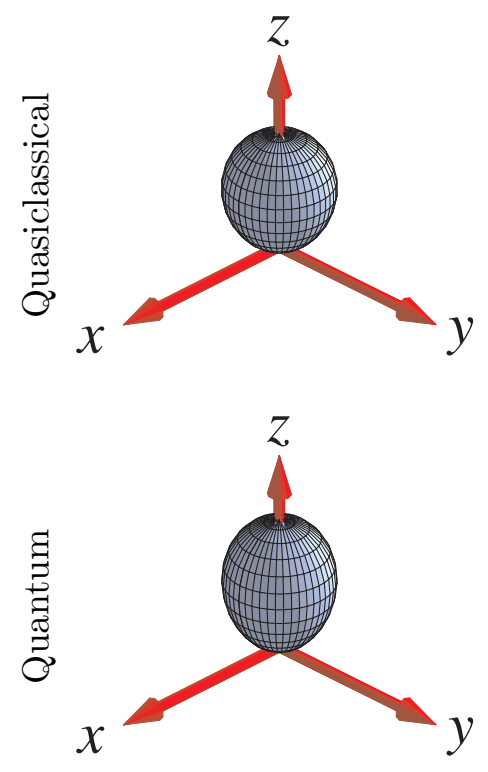

Fig. 2.1.: Quantum and quasiclassical PDFs associated with a $|j m\rangle$ angular momentum with $j=2$ and $m=2$.

means that the density operator describing the system will be given by

$$
\hat{\rho}=|22\rangle\langle 22|
$$

and that the polarisation moments are the following (obtained by inserting that density operator into Eq. (2.43))

$$
\begin{aligned}
& a_{0}^{(0)}=\langle 2200 \mid 22\rangle=1 \\
& a_{0}^{(1)}=\langle 2210 \mid 22\rangle=0.82 \\
& a_{0}^{(2)}=\langle 2220 \mid 22\rangle=0.53 \\
& a_{0}^{(3)}=\langle 2230 \mid 22\rangle=0.27 \\
& a_{0}^{(4)}=\langle 2240 \mid 22\rangle=0.09
\end{aligned}
$$

The classical PDF associated to a $|22\rangle$ state is

$$
P\left(\theta_{j}, \phi_{j}\right)=\frac{1}{2 \pi} \delta\left(\cos \theta_{j}-\frac{2}{\sqrt{2(2+1)}}\right)
$$

which, when substituted in Eq. (2.4), provides us with the following polarisation 
moments

$$
\begin{aligned}
& a_{0}^{(0)}=P_{0}\left(\frac{2}{\sqrt{2(2+1)}}\right)=1 \\
& a_{0}^{(1)}=P_{1}\left(\frac{2}{\sqrt{2(2+1)}}\right)=0.82 \\
& a_{0}^{(2)}=P_{2}\left(\frac{2}{\sqrt{2(2+1)}}\right)=0.50 \\
& a_{0}^{(3)}=P_{3}\left(\frac{2}{\sqrt{2(2+1)}}\right)=0.14 \\
& a_{0}^{(4)}=P_{4}\left(\frac{2}{\sqrt{2(2+1)}}\right)=-0.18
\end{aligned}
$$

with $P_{k}(\cos \theta)$ representing a Legendre polynomial (the classical list of moments do not finish with the $k=4$ one but continuous all the way to $k=\infty$. However, due to the Clebsch-Gordan coefficient presence, the classical moment with $k>4$ will not contribute to the quasiclassical PDF and, in consequence, they are not included in the list). In both cases it has been assumed that the system population is equal to one. Fig. 2.1 shows the result of inserting the quantum mechanical and the classical moments in Eq. (2.60). The quasiclassical PDF is in excellent agreement with its quantum counterpart, displaying only an small difference: the quasiclassical PDF is slightly broader than the quantum one. This feature is found in all cases and comes from the definition of the quantum PDF as a population distribution instead of as a probability distribution. 3D plots as Fig. 2.1 will be constantly used in the following Chapters as they sum up the characteristic of the angular momentum distribution in just a figure and, when quantum moments are used, they do it exactly.

To conclude the discussion about the quantum and quasiclassical PDFs it is important to point out two additional properties:

- They do not necessarily have to be prepared from moments corresponding to states with population one (as we did so far). In particular, if we were interested in using the PDFs to compare the angular momentum polarisation of systems or states with different population, it would be convenient to employ renormalised moments to generate them (see former Section). The role of the renormalised moments becomes transparent when considering that the PDFs volume coincides with the $k=0$ moment (the population). This involves (i) that the PDFs corresponding to different systems/states with different populations will differ in the volume and, eventually (if their $k \neq 0$ moments were different as well), also in the shape and (ii) that to employ renormalised moments removes the dif- 
ferences due to the population by resizing the PDFs (without being deformed) in such a way that their new volume is one.

- The correspondence principle limit $(j \gg k)$ of the Clebsch-Gordan coefficients involved in Eq. (2.60) is given by [39]

$$
\langle j j k 0 \mid j j\rangle \approx P_{k}\left(\frac{j}{\sqrt{j(j+1)}}\right) \approx P_{k}(1)=1
$$

It ensues from this expression that the quantum mechanical restriction represented by the Clebsch-Gordan coefficients vanishes in the classical limit and that, in consequence, the classical, the quasiclassical and the quantum PDFs become identical. 


\section{Atom-diatom collisions}

In any undergraduate book of Physical Chemistry there are several Chapters devoted to "Statistical Mechanics"; the area of the Physics which relates the Thermodynamical properties of a bulk system with the microscopic properties of its elementary constituents (atoms or molecules). Equally, elementary collisions are behind any chemical reaction and a detailed knowledge of their characteristics allows for the explanation of the macroscopic behaviour of the reaction and for the evaluation of any property related to it (in particular, those which will be used to study the reaction stereodynamics: the polarisation of the atoms and molecules participating in the reaction).

The aim of this Chapter is to introduce the concepts and methodology related to the study of (non-relativistic) gas phase collisions between atoms and diatomic molecules

$$
\mathrm{A}+\mathrm{BC} \rightarrow \mathrm{AB}+\mathrm{C}
$$

The idea is not to provide with a rigorous discussion of the scattering theory but to present the tools that will be necessary for the development and understanding of the theory contained in the following Chapters. It begins with the definition of the cross section (Sec. 3.1), an observable related to the probability of the reaction and that constitutes one of the most important pieces of information about it. The analysis of the elementary collisions and the subsequent evaluation of the cross section (or of any other reaction observable) can be performed by means of the quasiclassical trajectories method (Sec. 3.3) or by the quantum molecular scattering theory (Sec. 3.4) (although they are not the only procedures for theoretically studying chemical reactions, this work only will make use of them). These two methodologies will be presented from the adiabatic point of view (that is, considering that just a potential energy surface (Sec. $[3.2)$ ) is involved in the collisions). 


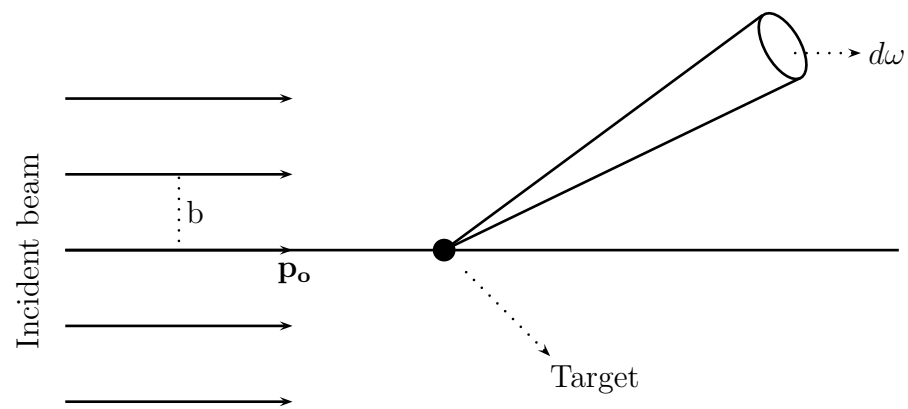

Fig. 3.1.: Collision of a beam of incident particles with a target at rest. The solid angle $d \omega$ represents the position of the detector, b represents the impact parameter and $\mathbf{p}_{\circ}$ the incident moment of the particles.

\subsection{Cross section}

Let us consider the arrangement shown in Fig. 3.1, where an incident beam of particles is directed towards and unit target and the scattered particles are measured by a detector placed along the direction defined by the $d \omega$ solid angle. Assuming that the incidents particles are projected with random impact parameters ${ }^{1}$ so that it is possible to speak of an uniform incident flux $I_{\text {inc }}$ (number of particles that, per unit time, cross an unit area perpendicular to the incident moment of the particles $\left.\left(\mathbf{p}_{\circ}\right)\right)$, the number of observed scattered particles into $d \omega$ per unit time will be given by

$$
d \dot{N}_{s c}=\frac{d \sigma}{d \omega} I_{\text {inc }} d \omega
$$

where $d \sigma / d \omega$ is called differential cross section (DCS). The DCS varies with the orientation of the detector to the incident beam and has dimensions of area per stereoradian. The detected particles can coincide with those forming the incident beam (elastic and inelastic scattering) or can be different (reactive scattering).

The integral cross section (ICS) is obtained by integrating the DCS over all the spatial directions

$$
\sigma=\int \frac{d \sigma}{d \omega} d \omega
$$

It has dimensions of area and represents the constant of proportionality between the

${ }^{1}$ The distance of closest approach of the colliding particles in the absence of interactions between them 


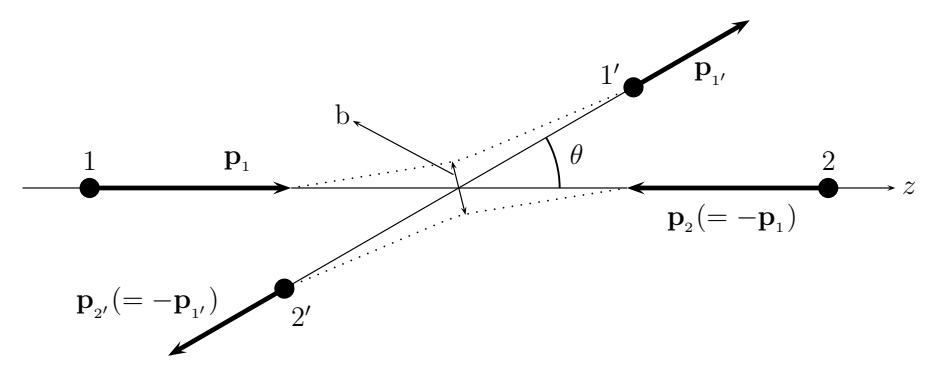

Fig. 3.2.: Collision between two particles $(1$ and 2$)$ in the centre-of-mass frame (the centre-of-mass is placed on the intersection of the continuous lines) that leads to formation of the particles $1^{\prime}$ and $2^{\prime}$ (depending on whether the process is reactive, inelastic or reactive the products will coincide with the reactants or not). The vectors $\mathbf{p}_{1}$ and $\mathbf{p}_{2}\left(\mathbf{p}_{1^{\prime}}\right.$ and $\left.\mathbf{p}_{2^{\prime}}\right)$ represent the linear momenta of the reactants (products), the $z$ axis is parallel to the relative momentum of the colliding particles, $\mathrm{b}$ is the impact parameter and $\theta$ is the scattering angle.

total number of scattered particles per unit time $\left(\dot{N}_{s c}\right)$ and the incident flux $\left(I_{\text {inc }}\right)$

$$
\dot{N}_{s c}=\sigma I_{\text {inc }}
$$

The former definitions hold regardless the choice of reference frame. For theoretical purposes, the most convenient one is that where the centre-of-mass is at rest (see Fig. 3.2); what is called the centre-of-mass (C.M.) frame. However, this is not the only possibility and one could consider a frame where the target particle is at rest (Fig. 3.1) or where both particles are moving. The integral cross section value does not depend on the frame of reference and the transformation of the differential cross section between different frames is a purely kinematic problem [51]. From now on and except otherwise stated, all the results will be referred to the centre-of-mass system.

There are several assumptions behind the cross sections definitions. One of them was previously indicated: the impact parameters are uniformly distributed in the plane perpendicular to $\mathbf{p}_{\circ}$. Fortunately, it is not necessary to send the particles over an infinite front but over a area that is large compared to the target size (the particles with sufficiently large impact parameters are not scattered ${ }^{2}$ ). Other underlying

\footnotetext{
${ }^{2}$ The scattering theory presented in this Chapter holds for potentials fulfilling certain conditions that can be found in refs. 51] 52, 53. One of those conditions focuses on the long-distance behaviour of the potential and, in particular, it sets that the potential must fall off quicker than $r^{-3}$ (where $r$ is the distance between the center of mass of the colliding particles). This statement is fulfilled by the intermolecular forces controlling chemical reactions between neutral species and, in consequence, it is meaningful to neglect the mutual influence of the collision participants when the distances are large and to say that collisions with very large impact parameters do not give
} 
assumptions are the following

- The forward elastic scattering ${ }^{3}$ is an undefined quantity because one can not distinguish a particle that is elastically scattered in the forward direction from a particle that is not scattered at all.

- Multiple scattering is negligible. This means that, for each incident particle, only two events can happen with a probability significantly different from zero: the particle can cross the target region without colliding or, alternatively, it can be scattered off by a single scattering center.

For a fixed energy, the differential cross section is a function of two angles: the scattering ( $\theta$ in Fig. 3.2. It is the angle formed by the relative velocity of colliding and recoiling particles) and the azimuthal ( $\phi$, measured with respect to any plane containing the relative velocity of the colliding particles (the $z$ axis in Fig. 3.2 ) ) angles. If the interaction potential only depends on the relative position of the particles (and not on any external parameter or direction) and if the colliding particles are initially unpolarised, the differential cross section will be function of $\theta$ but not of $\phi[1$. This is the case of most of the physical problems and, in particular, of the reactions studied in this work.

Regarding atom-diatom reactions, the state-to-state cross sections correspond to processes like

$$
\mathrm{A}+\mathrm{BC}(v, j) \rightarrow \mathrm{AB}\left(v^{\prime}, j^{\prime}\right)+\mathrm{C}
$$

that is, collisions where the reactants are prepared in well defined vibrational and rotational states (represented by the $v$ and $j$ quantum numbers) and the products are detected only when formed with well defined vibrational and rotational states (represented by the $v^{\prime}$ and $j^{\prime}$ quantum numbers). If, instead, the detection does not discriminate between different final states, the corresponding cross sections will be termed as total cross sections.

Although the cross section is not an intuitive concept and it could be thought to be very far from the "test tube" chemistry, the fact is that it allows for the theoretical evaluation of more "chemical" quantities as the thermal rate coefficients, providing with the desired connection between elementary collisions and bulk properties of the reaction. For instance, the state-to-state thermal rate coefficients for reaction 3.5 can

rise to scattered particles

${ }^{3}$ Scattering is said to be forward (backward) when the final relative velocity of the scattered particles is parallel (antiparallel) to the initial relative velocity of the colliding particles 
be expressed as [1, 54.

$$
\begin{aligned}
k_{v^{\prime} j^{\prime}, v j}(T) & =\int_{0}^{\infty} v_{r} \sigma_{v^{\prime} j^{\prime}, v j}\left(v_{r}\right) f\left(v_{r}\right) d v_{r} \\
& =\left(\frac{\mu}{2 \pi k_{B} T}\right)^{3 / 2} \int_{0}^{\infty} v_{r} \sigma_{v^{\prime} j^{\prime}, v j}\left(v_{r}\right) \exp ^{-\mu v_{r}^{2} / 2 k_{B} T} 4 \pi v_{r}^{2} d v_{r}
\end{aligned}
$$

where $v_{r}$ is the relative velocity of the reactants particles, $\sigma_{v^{\prime} j^{\prime}, v j}\left(v_{r}\right)$ is the integral cross section for reaction 3.5 (indicating explicitly its dependence on the collision energy (through the relative velocity)) and $f\left(v_{r}\right)$ represents the Maxwell-Boltzman distribution of relative speeds (its usage is consistent with the thermal equilibrium of the molecules at each temperature $T$ ). From $k_{v^{\prime} j^{\prime}, v j}(T)$ it is possible to evaluate state-averaged thermal rate coefficients [1, 54 that can be compared to experimental measurements.

\subsection{Born-Oppenheimer approximation}

The Schrödinger equation for a system formed by $N$ electrons and $M$ nuclei is given by 55 .

$$
(\hat{H}-E) \Psi(\mathbf{r}, \mathbf{R})=0
$$

where $E$ is the total energy, $\Psi(\mathbf{r}, \mathbf{R})$ is the system wavefunction ( $\mathbf{r}$ represents the coordinates of the $N$ electrons $\left(\mathbf{r}_{1}, \ldots, \mathbf{r}_{N}\right)$ and $\mathbf{R}$ represents the coordinates of the $M$ nuclei $\left.\left(\mathbf{R}_{1}, \ldots, \mathbf{R}_{M}\right)\right)$ and $\hat{H}$ is the Hamiltonian operator

$$
\hat{H}=-\frac{\hbar^{2}}{2 m_{e}} \sum_{i}^{N} \nabla_{r_{i}}^{2}-\frac{\hbar^{2}}{2} \sum_{A}^{M} \frac{1}{m_{A}} \nabla_{R_{A}}^{2}+\hat{V}(\mathbf{r}, \mathbf{R})
$$

whose different terms correspond respectively to the electronic kinetic energy $\left(m_{e}\right.$ is the electron mass), to the nuclear kinetic energy ( $m_{A}$ is the mass of the $A$ nucleus) and to the coulombic interaction between the different particles. The concrete expression for this potential is the following

$$
\hat{V}=\frac{e^{2}}{4 \pi \epsilon_{\circ}}\left[-\sum_{A}^{M} \sum_{i}^{N} \frac{Z_{A}}{r_{A i}}+\sum_{i, j>i}^{N} \frac{1}{r_{i j}}+\sum_{A, B>A}^{M} \frac{Z_{A} Z_{B}}{R_{A B}}\right]
$$

where $r_{A i}=\left|\mathbf{R}_{A}-\mathbf{r}_{i}\right|, r_{i j}=\left|\mathbf{r}_{i}-\mathbf{r}_{j}\right|, R_{A B}=\left|\mathbf{R}_{A}-\mathbf{R}_{B}\right|$ and the fist double sum represents the electron-nucleus attraction, the second the electron-electron repulsion and the third the nucleus-nucleus repulsion. 
All the information regarding the system (including that concerning the scattering states) is contained in Eq. (3.7). However, the solution of that equation is a complicated task that needs the employment of different approximations, being the most important one the Born-Oppenheimer (B.O.) approximation [56, 57]. It stems from the fact that $m_{A} \gg m_{e}$ and that, in consequence, the electrons change their position much faster than the nuclei. Based on this difference, the B.O. approximation provides with a method for solving Eq. (3.7) by assuming that the electrons can be considered as moving in the field caused by the fixed nuclei and, vice versa, that the nuclei do not "feel" the instantaneous positions of the electrons but their average. This involves that the electronic and nuclear movements can be separated and that the system wavefunction can be written, in its simplest formulation, as

$$
\Psi(\mathbf{r}, \mathbf{R})=\psi_{\mathrm{el}}(\mathbf{r} ; \mathbf{R}) \theta_{\text {nuc }}(\mathbf{R})
$$

where $\psi_{\mathrm{el}}(\mathbf{r} ; \mathbf{R})$ is the electronic wavefunction (that notation means that it depends explicitly on the electronic coordinates $\mathbf{r}$ and parametrically on the nuclear coordinates $\mathbf{R}$ ) and $\theta_{\text {nuc }}(\mathbf{R})$ is the nuclear wavefunction. The first describes the electronic state and it is determined by separately solving the electronic Schrödinger equation for each nuclear geometry. The eigenvalue of this equation, together with the nuclear repulsion, is termed as potential energy surface (PES). The PES plays the role of the potential energy for the nuclear movement, which is described by the wavefunction $\theta_{\text {nuc }}(\mathbf{R})$ obtained from the nuclear Schrödinger equation. Explicit expressions for this equation and its electronic counterpart are included in the following paragraphs.

The electronic Schrödinger equation describing the behaviour of the electrons in the framework of the B.O. approximation is given by

$$
\left(\hat{H}_{\mathrm{el}}-E_{\mathrm{el}}(\mathbf{R})\right) \psi_{\mathrm{el}}(\mathbf{r} ; \mathbf{R})=0
$$

where $\hat{H}_{\mathrm{el}}$ is the electronic Hamiltonian

$$
\hat{H}_{\mathrm{el}}=-\frac{\hbar^{2}}{2 m_{e}} \sum_{i}^{N} \nabla_{r_{i}}^{2}-\frac{e^{2}}{4 \pi \epsilon_{\circ}} \sum_{A}^{M} \sum_{i}^{N} \frac{Z_{A}}{r_{A i}}+\frac{e^{2}}{4 \pi \epsilon_{\circ}} \sum_{i, j>i}^{N} \frac{1}{r_{i j}}
$$

It differs from $\hat{H}$ (Eq. (3.8)) on two terms: the nuclear kinetic energy (second term in Eq. (3.8)) and the coulombic interaction between nuclei (third term in Eq. (3.9)). It is clear that, under the approximation assumptions, both quantities can be neglected when considering the electrons movement as the first is zero for any fixed nuclear configuration and the second is just a constant. The electronic energy is represented 
by $E_{\mathrm{el}}(\mathbf{R})$ and, as the electronic wavefunction, depends parametrically on the nuclear coordinates.

In order to complete the solution of Eq. (3.7), it is still necessary to tackle the problem of the nuclear motion. The B.O. separation between electronic and nuclear motions implies that, when considering the nuclear dynamics, the electronic coordinates can be substituted by their averaged values, defining this way the nuclear Hamiltonian

$$
\begin{aligned}
\hat{H}_{\mathrm{nuc}} & =-\frac{\hbar^{2}}{2} \sum_{A}^{M} \frac{1}{m_{A}} \nabla_{R_{A}}^{2}+\underbrace{\frac{1}{4 \pi \epsilon_{0}}\left\langle-\frac{\hbar^{2}}{2 m_{e}} \sum_{i}^{N} \nabla_{r_{i}}^{2}-\sum_{A}^{M} \sum_{i}^{N} \frac{Z_{A}}{r_{A i}}+\sum_{i, j>i}^{N} \frac{1}{r_{i j}}\right.}_{E_{\mathrm{el}}(\mathbf{R})}\rangle \\
\frac{1}{4 \pi \epsilon_{0}} \sum_{A, B>A}^{M} \frac{Z_{A} Z_{B}}{R_{A B}} & =-\frac{\hbar^{2}}{2} \sum_{A}^{M} \frac{1}{m_{A}} \nabla_{R_{A}}^{2}+\underbrace{E_{\mathrm{el}}(\mathbf{R})+\frac{1}{4 \pi \epsilon_{0}} \sum_{A, B>A}^{M} \frac{Z_{A} Z_{B}}{R_{A B}}}_{U(\mathbf{R})} \\
& =-\frac{\hbar^{2}}{2} \sum_{A}^{M} \frac{1}{m_{A}} \nabla_{R_{A}}^{2}+U(\mathbf{R})
\end{aligned}
$$

where the potential energy role is played by $U(\mathbf{R})$ (the total energy for fixed nuclei) and the terms consisting of integrals like

$$
\left\langle\psi_{\mathrm{el}}(\mathbf{r} ; \mathbf{R})\left|\nabla_{R_{A}}\right| \psi_{\mathrm{el}}(\mathbf{r} ; \mathbf{R})\right\rangle=\int \psi_{\mathrm{el}}^{*}(\mathbf{r} ; \mathbf{R}) \nabla_{R_{A}} \psi_{\mathrm{el}}(\mathbf{r} ; \mathbf{R}) d \mathbf{r}
$$

and

$$
\left\langle\psi_{\mathrm{el}}(\mathbf{r} ; \mathbf{R})\left|\nabla_{R_{A}}^{2}\right| \psi_{\mathrm{el}}(\mathbf{r} ; \mathbf{R})\right\rangle=\int \psi_{\mathrm{el}}^{*}(\mathbf{r} ; \mathbf{R}) \nabla_{R_{A}}^{2} \psi_{\mathrm{el}}(\mathbf{r} ; \mathbf{R}) d \mathbf{r}
$$

have been discarded (when working in the context of the B.O. approximation, the electronic function is expected to change slowly with the nuclear coordinates).

The nuclear Schrödinger equation

$$
\left(\hat{H}_{\mathrm{nuc}}-E\right) \theta_{\mathrm{nuc}}(\mathbf{R})=0
$$

allows for determining the B. O. approach to the total energy $E$ (Eq. (3.7)) and the nuclear wavefunction $\theta_{\text {nuc }}(\mathbf{R})$ which describes the behaviour of the nuclei and can be used to study: (i) the rotations, vibrations and displacements of the system and (ii) the scattering properties (if the system is not a bounded one).

If only the relative motion of the $M$ nuclei is considered (by taking away the displacements and the rotations of the system as a whole), their geometry can be 
specified by means of $3 M-6$ independent variables. Therefore, $U(\mathbf{R})$ is a function which depend on the same variables and whose graphical representation consists of a $3 M-6$ dimensional surface; the potential energy surface (PES).

Phenomena and processes such as internal rotational barriers, dissociation, molecular dynamics, molecular scattering and infrared and microwave spectroscopy have a simple, intuitive and reliable interpretation based on the B.O. approximation and, in particular, on the PES concept [58], which is absolutely crucial for the Physical Chemistry.

However, it is necessary to point out that, in spite of this ubiquitous and successful usage, the B.O. treatment is not always acceptable [59, 60] as there are physical and chemical phenomena where the separation between the electronic and the nuclear movements can not be performed because all the particles forming the system are strongly correlated. Examples of these phenomena are electronically excited states (Rydberg states), muonic molecules and all those nuclear configurations where two different PES approach and, eventually, cross (photochemistry is almost exclusively based on these crossings).

To cope with these situations it is necessary to use alternative techniques. The adiabatic representation consists of expanding the molecular wave function as

$$
\Psi(\mathbf{r}, \mathbf{R})=\sum_{n} \theta_{\mathrm{nuc}, n}(\mathbf{R}) \psi_{\mathrm{el}, n}(\mathbf{r} ; \mathbf{R})
$$

where $\psi_{\mathrm{el}, n}$ represents an electronic eigenfunction and the expansion coefficients $\theta_{\mathrm{nuc}, n}$ depend on the nuclear coordinates. Alternatively, the diabatic representation employs as expansion basis the electronic eigenfunctions evaluated at a single nuclear geometry $\left(\mathbf{R}_{0}\right)$

$$
\Psi(\mathbf{r}, \mathbf{R})=\sum_{n} \theta_{\text {nuc }, n}^{\prime}(\mathbf{R}) \psi_{\mathrm{el}, n}\left(\mathbf{r} ; \mathbf{R}_{0}\right)
$$

The employment of these series allow for transforming the molecular Schrödinger equation (3.7) into a set of coupled differential equations for the nuclear functions $\theta_{\text {nuc }, n}(\mathbf{R})$ and $\theta_{\text {nuc }, n}^{\prime}(\mathbf{R})$. The properties of such set depend on the explicit form of the expansion and are related to the advantages and disadvantages of each representation:

- The set of equations derived from the diabatic expansion include the coupling terms in the potential energy, in contrast with the adiabatic case, where the coupling appears in the kinetic energy term. This is a point in favour of the diabatic representation, as it makes calculations easier.

- The electronic functions employed in the diabatic representation are not eigen- 
functions of the electronic hamiltonian for all the nuclear geometries $\mathbf{R}$ but only for $\mathbf{R}_{0}$. This turns out to be an inconvenience that gives advantage to the adiabatic representation.

In the daily practise of reaction dynamics, non-adiabatic time independent calculations use to employ the adiabatic representation and non-adiabatic time dependent calculations the diabatic one ${ }^{4}$. To neglect the couplings terms in the system of equations for the nuclear functions is equivalent to take a single term in the series (3.17) and (3.18) and leads to the Born-Oppenheime ${ }^{5}$ and the diabatic approaches respectively. This last approximation holds for situations where the nuclear kinetic energy is larger than the electrons-nuclei interaction, in such a way that the electrons can not "follow" the nuclear motion (it is interesting to point out that these conditions are opposite to those suitable for the Born-Oppenheimer approximation).

The present work will consider the stereodynamics of chemical reactions from the point of view of the adiabatic approximation. The first step, prior to any scattering calculation, consists of determining the PES. Its values are worked out for a set of nuclear geometries that covers all the possible nuclear configurations (although treating with more detail those regions on the surface which will be crucial for the process, that is, the entrance and output valleys and the transition state region) and the resulting energies are fitted to an analytic function. There are two different approaches to the solution of the electronic equation at each nuclear geometry: ab initio and semiempirical methods. The first solves the electronic Schrödinger equation just by making an assumption for the electronic wavefunction (that will determine the accuracy of the result) and by using the values of the fundamental constants and the nuclear masses. Although more accurate, ab initio calculations are too computationally expensive for large molecular systems and, in practise, they are substituted by semiempirical calculations. This set of methods reduce the computational requirements by employing simplifications of the Hamiltonian together with some experimental data.

When the PES is known, it is possible to tackle the dynamical problem itself. The rest of the Chapter will concentrate on this task.

\footnotetext{
${ }^{4}$ Switching between both representations is always possible through an unitary transformation, provided that the basis sets are complete

${ }^{5}$ Strictly speaking, considering of only one term in Eq. (3.17) leads to the adiabatic approach, which differs from the Born-Oppenheimer approximation. However, this difference can generally be disregarded so that "adiabatic approximation" and "Born-Oppenheimer approximation" are usually employed interchangeably
} 


\subsection{Quasiclassical trajectory method}

Theories for the study of reactive molecular collisions can be divided into two different groups: dynamical and statistical methods. The former require to solve (exactly or approximately) the nuclear Schrödinger equation (quantum mechanical dynamics) or the Hamilton equations (classical dynamics). The second avoids the direct analysis of the collision dynamics by invoking suitable statistical considerations (the transition state theory $61,62,63$ is a paradigmatic example of these methods).

Dynamical calculations are necessary for the purposes of this work and, therefore, our attention will be exclusively focused on them: the general features of the Quasiclassical Trajectory (QCT) method 64, 65, 66, 67 are now presented, postponing the description of quantum mechanical calculations till next Section.

In the $\mathrm{QCT}$ context, a trajectory is the set of phase-space points representing the classical movement of the nuclei (with the potential energy given by the PES introduced in the former Section) taking part in a collision. The calculation of each trajectory starts by fixing the initial conditions of the system (this is equivalent to select a defined point in the phase space), continues by integrating the Hamilton equations which define the temporal trajectory of that point and concludes with the analysis of the products and the assignation of a quantized internal state to the products fragments. When this procedure is performed for a number of trajectories large enough to provide convergence, it is possible to obtain the state-to-state magnitudes which characterise the reaction (for instance, the cross sections defined in Sec. 3.1 or the polarisation moments introduced in next Chapter).

Given a $M$ particles system, its classical dynamics is given by the Hamilton's equations, which consist of a system of $6 M$ coupled equations 68

$$
\begin{aligned}
\dot{q}_{i}=\frac{\partial H}{\partial p_{i}} & i=1, \ldots, 3 M \\
\dot{p}_{i}=-\frac{\partial H}{\partial q_{i}} & i=1, \ldots, 3 M
\end{aligned}
$$

where $q(p)$ are the generalised coordinates (generalised moments), the dot indicates the first derivative with respect to time and $H$ is the Hamiltonian function. For a conservative system, $H$ is independent on time and its value is equivalent to the energy $(E)$ of the system

$$
H(q, p)=T(q, p)+V(q, p)=E
$$

being $T$ the kinetic and $V$ the potential energy (the PES). 


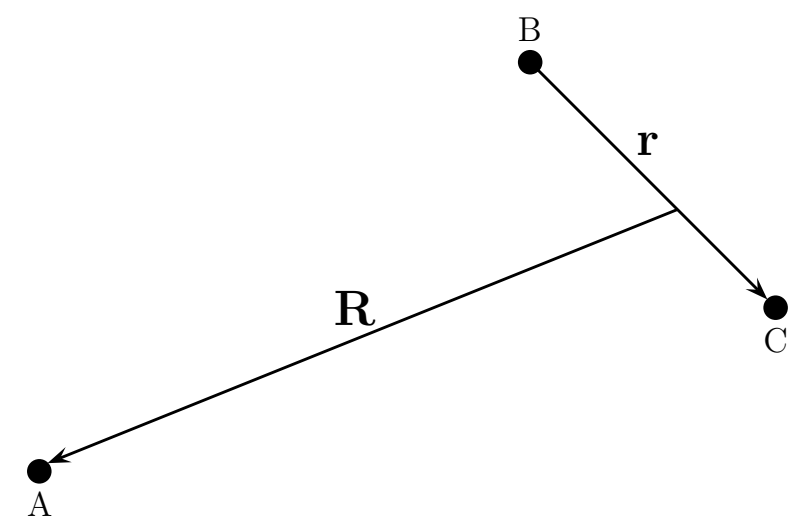

Fig. 3.3.: Reactants Jacobi coordinates. The $\mathbf{r}$ vector represents the $\mathrm{BC}$ internuclear axis and $\mathbf{R}$ is the vector connecting the $\mathrm{BC}$ centre-of-mass to $\mathrm{A}$.

The QCT calculations included in this work (see [66, 67] and references therein for a more exhaustive description regarding all aspects of the theory) concentrate on state-to-state atom-diatom reactions like (3.5) and employ the following coordinates and reference frame

- For a three particles system there are eighteen Hamilton's equations. However, this number can be reduced to twelve if the translation of the system as a whole is ignored. We implement such reduction by using Jacobi coordinates (Fig. 3.3), so that the components of $\mathbf{R}, \mathbf{r}$ and of the corresponding momenta will play the role of generalised coordinates and momenta in the resulting twelve equations.

- We adopt a frame of reference such that the origin of coordinates coincides with the centre-of-mass of the reactants diatomic molecule (BC), the incident atom is placed on the $y z$ plane and the $z$ value for the incident atom is negative, indicating that the relative velocity between the colliding particles is parallel to the positive $z$ axis.

Eqs. (3.19) and (3.20) are first order differential equations and, as such, they need as many initial conditions as equations in order to be integrated. The first step in the calculation of a trajectory consists of setting the initial values of the (twelve) coordinates and momenta. The choice of the frame of reference together with the conservation of energy (consequence of the time independence of the Hamiltonian) allows for expressing the initial conditions in terms of five geometric parameters:

- The polar angles $\left(\theta_{j}\right.$ and $\left.\phi_{j}\right)$ which determine the position of the $\mathrm{BC}$ rotational angular momentum $(\mathbf{j})$ with respect to the reference frame. 
- An angle $\eta$ whose value sets the position of the $\mathrm{BC}$ internuclear axis on the plane perpendicular to $\mathbf{j}$.

- The impact parameter $(b)$.

- The BC vibrational phase $(\chi)$. In order to ensure random selection (see below) of $\chi$ for different trajectories, the normal procedure is to set the initial value of the $\mathrm{BC}$ distance $\left(r_{0}\right)$ equal to one of the classical turning points and to add a random quantity to $R_{0}$ (the initial modulus of $\mathbf{R}$; its value is such that the atom-diatom interaction can be neglected). In this way, when the equation integration begins and the distance between $\mathrm{A}$ and the $\mathrm{BC}$ centre-of-mass reaches $R_{0}$, the vibrational phase of $\mathrm{BC}$ will be random. The turning points employed to determine $r_{0}$ are those which correspond to the quantum mechanical energy of the $v-j$ state $^{6}$. The initial energy value consist of two different contributions: the collision energy (the relative kinetic energy between the colliding particles) and the internal energy of the $\mathrm{BC}$ molecule. There is no contribution from the interaction between $\mathrm{A}$ and $\mathrm{BC}$ because $R_{0}$ is large enough to neglect it. While the value of the collision energy is initially fixed for each trajectory, the internal energy is chosen as the quantum mechanical energy corresponding to the $v-j$ state. The turning points corresponding to this energy are identified and then used to set $r_{0}$.

When the energy and the five parameters are fixed, it is possible to evaluate the initial values of the variables and the Hamilton's equations can be integrated by means of any of the standard procedures developed for solving systems of coupled differential equations [66, 69]. Integration continues till one of the atom-atom distances exceeds a certain cut-off value and, when this happens, the trajectory is assumed to be completed and the products diatomic molecule is identified. At this point it is possible to determine whether the trajectory is reactive or inelastic/elastic. Differentiation between these last two types of trajectories will not be possible until completing the products analysis.

The following (and last) step consists of expressing the positions and momenta of the atoms in terms of the Jacobi products coordinates $\left(\mathbf{R}^{\prime}\right.$ and $\mathbf{r}^{\prime}$. Defined as their reactants counterparts: $\mathbf{r}^{\prime}$ is the $\mathrm{AB}$ internuclear axis and $\mathbf{R}^{\prime}$ the vector joining the $\mathrm{AB}$ centre-of-mass to $\mathrm{C}$ ) and of the subsequent analysis of the products, that is, the determination of (i) their relative translational, vibrational and rotational energies,

${ }^{6}$ Evaluated for each diatomic molecule participating in the reaction $(\mathrm{AB}, \mathrm{BC}, \mathrm{AC})$ by solving the Schrödinger equation in the corresponding asymptotes of the PES. These values are calculated prior to the trajectory analysis and fitted by means of a Dunham expansion 
(ii) the rotational angular momentum of $\mathrm{AB}$ and (iii) the scattering angle. With these data it is possible to check if the energy conservation is being fulfilled and to carry out the assignment of final quantum numbers $\left(v^{\prime}\right.$ and $j^{\prime}$, see reaction (3.5)) by means of the following equations

$$
\widetilde{j}^{\prime}=-\frac{1}{2}+\frac{1}{2} \sqrt{1+\frac{4 \mathbf{j}_{\mathrm{AB}} \mathbf{j}_{\mathrm{AB}}}{\hbar^{2}}}
$$

and

$$
\widetilde{v}^{\prime}=-\frac{1}{2}+\frac{1}{\pi \hbar} \int_{r_{-}}^{r_{+}} \sqrt{2 \mu_{\mathrm{AB}}\left(E_{\mathrm{int}}(\mathrm{AB})-V\left(r_{\mathrm{AB}}\right)-\frac{\mathbf{j}_{\mathrm{AB}} \mathbf{j}_{\mathrm{AB}}}{2 \mu_{\mathrm{AB}} r_{\mathrm{AB}}^{2}}\right)} d\left(r_{A B}\right)
$$

where $\mathbf{j}_{A B}$ is the rotational angular momentum of the products diatomic molecule $(\mathrm{AB}), E_{\text {int }}(\mathrm{AB})$ its internal energy, $\mu_{\mathrm{AB}}$ its reduced mass, $V\left(r_{\mathrm{AB}}\right)$ the potential energy corresponding to the $\mathrm{AB}$ asymptote of the PES and $r_{ \pm}$the classical turning points numerically evaluated for $E_{\text {int }}(\mathrm{AB})$. The values of $\widetilde{v}^{\prime}$ and $\widetilde{j}^{\prime}$ so obtained are real numbers that, when rounded to the nearest integer, provide with the values of $v^{\prime}$ and $j^{\prime}$.

The information derived from a single trajectory does not suitably represent the chemical reaction because it corresponds to a single set of initial conditions (a point in the phase space). On the contrary, typical experiments sample a wide range of initial conditions and, in order to properly simulate them, it will be necessary to integrate a large number of trajectories. The problem lies in that it is not possible to have an a priori knowledge of what are the suitable initial conditions that have to be used and, in consequence, the employment of an statistical sampling becomes necessary to ensure that the reaction attributes evaluated by means of the trajectories are representative.

Having calculated an statistically significant sample of trajectories, each one of them analysed in order to know the relevant quantities (scattering angle, translational and internal energy of the products, final quantum numbers, position of the rotational angular momenta of the diatomic molecules involved in the collision, etc), it is possible to derive the corresponding reaction properties which can be compared with experimental measurements or with results obtained through QM methods. In particular, among all the reaction properties that can be evaluated by means of the QCT method, our attention will be focused now on the state-to-state integral and differential reactive cross sections. At a well defined value of the energy, the former 
is given by

$$
\sigma_{v^{\prime} j^{\prime} \leftarrow v j}=\pi b_{\max }^{2} \frac{N_{v j v^{\prime} j^{\prime}}}{N}
$$

where $b_{\max }$ is the maximum reactive impact parameter (determined prior to the calculations by running an small number of trajectories), $N$ is the total number of trajectories calculated and $N_{v j v^{\prime} j^{\prime}}$ is the number of reactive trajectories taking to products in the $v^{\prime}, j^{\prime}$ state. The error of Eq. (3.24) can be estimated by means of the expression

$$
\Delta \sigma_{v^{\prime} j^{\prime} \leftarrow v j}=\pi b_{\max }^{2} \frac{N_{v j v^{\prime} j^{\prime}}}{N}\left(\frac{N-N_{v j v^{\prime} j^{\prime}}}{N N_{v j v^{\prime} j^{\prime}}}\right)^{1 / 2}
$$

Regarding the differential cross section, its value in the centre-of-mass frame can be calculated through an expansion in Legendre polynomials $\left(P_{k}(\cos \theta)\right)$

$$
\frac{d \sigma_{v^{\prime} j^{\prime} \leftarrow v j}}{d \omega}(\theta)=\frac{\sigma_{v^{\prime} j^{\prime} \leftarrow v j}}{2 \pi} \sum_{k=0}^{\infty} \frac{2 k+1}{2} s_{k} P_{k}(\cos \theta)
$$

with $\theta$ representing the centre-of-mass scattering angle. The coefficients $s_{k}$ are given by

$$
s_{k}=\frac{1}{N_{v j v^{\prime} j^{\prime}}} \sum_{i=1}^{N_{v j v^{\prime} j^{\prime}}} P_{k}\left(\cos \theta_{i}\right)
$$

where the sum runs over all the reactive trajectories leading to a certain final state $v^{\prime}, j^{\prime}$ and $\theta_{i}$ is the scattering angle corresponding to each trajectory.

\subsection{Quantum reactive scattering}

\section{Channels}

A channel is any set of stable (elementary or composite) particles that can enter or leave a collision 51, 52, 53.

To illustrate this concept, let us suppose a system formed by three particles $a, b$ and $c$ such that $b$ and $c$ have two bounded states (a ground state $(b c$ ) and an excited state $\left.\left(b c^{*}\right)\right)$ and $a$ and $c$ have an unique bounded state $a c$. The four possible channels 


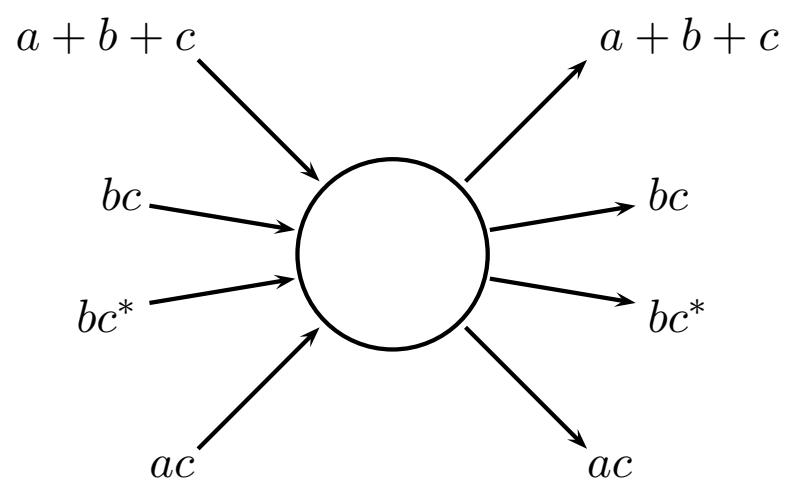

Fig. 3.4.: Schematic representation of a multichannel scattering process.

for any collision involving these particles are

$$
\begin{aligned}
& \text { Channel } 1 \Rightarrow a+b+c \\
& \text { Channel 2 } \Rightarrow a+b c \\
& \text { Channel 3 } \Rightarrow a+b c^{*} \\
& \text { Channel } 4 \Rightarrow b+a c
\end{aligned}
$$

where the first corresponds to the free movement of all particles and the rest of them to bounded states of the particles taking part in the collision. As it can be noticed, different channels differentiate not only in the fragments but also in the energy level of these fragments. It is not necessary to consider bounded states involving $a, b$ and $c$ simultaneously because these are eigenstates of the system Hamiltonian which remain bounded at all times.

A multichannel collision could be represented through the flux diagram 3.4. The colliding particles, whose state can correspond to a superposition of several channels or (more frequently) to a definite one, undergo the collision (the circle indicates the interaction region) and the products leave through several channels in definite proportions.

In principle, the number of channels can be either finite or infinite. However, not all of them are accessible at each value of the total energy $E^{7}$ : only those channels such that the internal energy of its constituents is below $E$ can represent states of the system prior or after the collision. These channels are termed as "opened channels"

\footnotetext{
${ }^{7}$ Whenever the potential describing the particles interaction is independent on time, the total energy has to be conserved during the collision
} 
and its number increases with the energy.

In this work, states differing in the angular momentum polarisation will be considered as belonging to the same channel. For instance, let us suppose that $b c$ represents a diatomic molecule. In this case, states corresponding to different polarisations of the rotational angular momentum and to the same internal state of the molecule will be included in the same channel.

\section{Scattering operator}

Let us suppose that out purpose is to study a $K$ particles system and, in particular, the scattering processes involving those particles and/or composite fragments formed with them. It can be shown [51, 52, 53] that the asymptotic ${ }^{8}$ state of the system prior and after any collision can be described through the coherent or incoherent superposition of state vectors belonging to a Hilbert space $\mathbb{H}$ that can be decomposed as the direct sum

$$
\mathbb{H}=\mathbb{H}_{1} \oplus \mathbb{H}_{2} \oplus \ldots \oplus \mathbb{H}_{\mathrm{N}}
$$

where $N$ is the number of channels (in practise only opened channels have to be considered) and each $\mathbb{H}_{i}$ represents the Hilbert space formed by the vectors suitable for describing the arrangement corresponding to channel $i$.

To clarify this, let us go back to the former example. If $a, b$ and $c$ were spinless particles, $\mathbb{H}_{1}$ (channel 1 is that corresponding to the free movement of all particles) turns out to be $\mathcal{L}^{2}\left(\mathbb{R}^{9}\right.$ ) (the space of square-integrable functions in $\mathbb{R}^{9}$ ) and the coordinate representation of an arbitrary vector $\left|\Psi_{1}\right\rangle$ of this space is

$$
\left\langle\mathbf{x}_{a}, \mathbf{x}_{b}, \mathbf{x}_{c} \mid \Psi_{1}\right\rangle=\Psi_{1}\left(\mathbf{x}_{a}, \mathbf{x}_{b}, \mathbf{x}_{c}\right)
$$

In contrast with this, $\mathbb{H}_{2}$ consist of the subspace of $\mathcal{L}^{2}\left(\mathbb{R}^{9}\right)$ formed by the vectors whose coordinate representation is given by

$$
\left\langle\mathbf{x}_{a}, \mathbf{x}_{b}, \mathbf{x}_{c} \mid \Psi_{2}\right\rangle=\chi\left(\mathbf{x}_{a}, \overline{\mathbf{x}}_{b c}\right) \phi_{b c}\left(\mathbf{x}_{b c}\right)
$$

where $\chi\left(\mathbf{x}_{a}, \overline{\mathbf{x}}_{b c}\right)$ is an arbitrary normalizable function describing the movement of $a\left(\mathbf{x}_{a}\right)$ and the $b c$ center of mass $\left(\overline{\mathbf{x}}_{b c}\right)$ and $\phi_{b c}\left(\mathbf{x}_{b c}\right)$ is the function representing the internal motion $\left(\mathbf{x}_{b c}\right)$ of the $b c$ bounded state. This could be extended to channels 3 and 4 in an straightforward way. The space $\mathbb{H}$ formed by the vectors describing

\footnotetext{
${ }^{8}$ Each collision considered in this work can be divided into three parts: in asymptote, interaction region and out asymptote. The asymptotes are characterised by the "free" movement of the system, where "free" indicates now that the interaction between the particles and fragments of each opened channel can be neglected
} 
the system state before or after the collision would be obtained from $\mathbb{H}_{i}(i=1, \ldots, 4)$ according to Eq. (3.28), with the direct sum indicating that a general state of the colliding (or recoiling) particles would include a certain contribution coming from all channels.

Having introduced $\mathbb{H}$, it is time now for presenting the most important element of the quantum mechanical scattering theory: the scattering operator $\hat{S}$. It is

- an unitary operator defined on $\mathbb{H}$, that is, a linear operator whose domain is $\mathbb{H}$, whose range is $\mathbb{H}$ and that conserves the norm (the first two conditions involve that $\hat{S}$ is a one-to-one map and, therefore, that it can be inverted)

- such that if the system state prior to collision is given by $\left|\Psi^{\mathrm{in}}\right\rangle$, the state which describes it when the collision has taken place is

$$
\left|\Psi^{\text {out }}\right\rangle=\hat{S}\left|\Psi^{\text {in }}\right\rangle
$$

where both $\left|\Psi^{\text {in }}\right\rangle$ and $\left|\Psi^{\text {out }}\right\rangle$ belong to $\mathbb{H}$.

Most commonly, the in-state lies in one definite channel $i$ and, hence,

$$
\left|\Psi^{\mathrm{in}}\right\rangle=\left|\Psi_{i}\right\rangle
$$

However, the out-state will in general include contributions from all the opened channels even although the in-channel is unique. If this were not so, the inelastic and the reactive scattering would not be possible and all the collisions would consist of elastic processes without internal energy transference or particle rearrangement.

\section{Interpretation of the elements of the S matrix. Scattering matrix}

The probability amplitude for a process consisting of a collision where the system enters with state $|\Psi\rangle$ and leaves with state $\left|\Psi^{\prime}\right\rangle$ is given by

$$
\left\langle\Psi^{\prime}|\hat{S}| \Psi\right\rangle
$$

that is, by the overlapping between $\left|\Psi^{\prime}\right\rangle$ and $\hat{S}|\Psi\rangle$. The probability for that process is nothing but the square of the amplitude

$$
P\left(\Psi^{\prime} \leftarrow \Psi\right)=\left|\left\langle\Psi^{\prime}|\hat{S}| \Psi\right\rangle\right|^{2}
$$

Complex numbers like (3.33) are the constituents of the scattering matrix ${ }^{9}$ (S ma-

\footnotetext{
${ }^{9}$ Strictly speaking, the scattering matrix would be formed by the $\left\langle n|\hat{S}| n^{\prime}\right\rangle$ elements, where $|n\rangle$ and
} 
trix): an unitary matrix (because the scattering operator is unitary) whose elements are determined by the dynamics of the collision and that completely characterizes the scattering process. As it will be showed in the rest of this Section and in the following Chapter, its knowledge allows for evaluating any collision observable.

\section{Representations of the scattering matrix. Helicity representation}

The former formalism can be applied to the theoretical study of atom-diatom reactions and, in particular, to state-to-state processes like

$$
\mathrm{A}+\mathrm{BC}(v, j) \rightarrow \mathrm{AB}\left(v^{\prime}, j^{\prime}\right)+\mathrm{C}
$$

The potential controlling the nuclear movement in these reactions is given by a PES (only adiabatic processes are considered) that accounts for the electronic energy and the nuclear repulsion and whose nature and properties were presented in Sec. 3.2 , It ensues from those properties that the PES values are independent of time and invariant under rotations and coordinates inversion (because they only depend on the relative geometry of the nuclei). In consequence [70,

- the total energy $(E)$

- the total angular momentum quantum number $(J)$ and its projection along any space fixed axis $(M)$ and

- the parity $(P)$

are conserved during a collision and the elements of the $\mathrm{S}$ matrix can be represented as

$$
S_{a^{\prime}, a}^{E J M P}
$$

where $a$ and $a^{\prime}$ label the asymptotic states, that is, the system state prior and after the collision.

In principle, one could perform this labelling in infinite different manners (one for each possible basis of $\mathbb{H}$ ) that determine infinite representations for the scattering matrix. Among them, the "orbital angular momentum" and the "helicity" representations $[71,72,73$, are widely employed, being the second most suitable for the study of reaction stereodynamics. The elements of the $\mathrm{S}$ matrix in the orbital angular momentum representation read

$$
S_{\alpha^{\prime} v^{\prime} j^{\prime} l^{\prime}, \alpha v j l}^{E J M P}
$$

$\left|n^{\prime}\right\rangle$ are vectors belonging to a basis of $\mathbb{H}$. If those elements are known it is possible to evaluate any other one corresponding to arbitrary ket and bra in $\mathbb{H}$ 
while in the helicity representation they are written as

$$
S_{\alpha^{\prime} v^{\prime} j^{\prime} \Omega^{\prime}, \alpha v j \Omega}^{E J M P}
$$

where unprimed and primed quantities refer to entrance and exit channel respectively, $\alpha$ and $\alpha^{\prime}$ represent the initial and final atomic arrangements, $v$ and $j\left(v^{\prime}\right.$ and $\left.j^{\prime}\right)$ are the vibrational and rotational quantum numbers of the diatomic molecule $\mathrm{BC}(\mathrm{AB})$, $l\left(l^{\prime}\right)$ is the orbital angular momentum quantum number for reactants (products) and $\Omega\left(\Omega^{\prime}\right)$ is the projection of $\mathbf{J}$ or $\mathbf{j}\left(\mathbf{j}^{\prime}\right)$ on $\mathbf{R}\left(\mathbf{R}^{\prime}\right)$ ( $\mathbf{J}$ is the total angular momentum, $\mathbf{j}$ $\left(\mathbf{j}^{\prime}\right)$ is the rotational angular momentum of $\mathrm{BC}(\mathrm{AB})$ and $\mathbf{R}\left(\mathbf{R}^{\prime}\right)$ is the Jacobi vector pointing from the diatom centre-of-mass onto the atom (Fig. 3.3) for the reactants (products) arrangement).

Conversion of the $\mathrm{S}$ matrix between different representations can be performed through unitary transformations [71, 72]. The explicit expression for the transformation between orbital angular momentum and helicity representations is

$$
S_{\alpha^{\prime} v^{\prime} j^{\prime} \Omega^{\prime}, \alpha v j \Omega}^{E J M P}=\sum_{l l^{\prime}} G_{l \Omega}^{J j} S_{\alpha^{\prime} v^{\prime} j^{\prime} l^{\prime}, \alpha v j l}^{E J M P} G_{l^{\prime} \Omega^{\prime}}^{J j^{\prime}}
$$

where the sum runs over the allowed values of $l$ and $l^{\prime}$ for each parity value ${ }^{10}$ and the coefficients of the transformation are given by

$$
G_{l \Omega}^{J j}=(-1)^{j+\Omega}\langle j \Omega J-\Omega \mid l 0\rangle\left[\frac{2}{1+\delta_{\Omega 0}}\right]^{1 / 2}
$$

It is important to point out that different authors employ different phase conventions when define the elements of the S matrix. Correspondingly, it is possible to find several expressions for the transformation coefficients $G_{l \Omega}^{J j}$ (see for example [74]) that differ in a phase. This is not problem as long as a coherent criteria for the phase is kept all along the calculations. In future Sections, only two features of the transformation, which hold regardless the concrete expression for the coefficients, will be invoked: its unitary character (that ensures probability conservation) and the presence of the Clebsch-Gordan coefficients.

In practise, it is possible to do without some of the quantum numbers that label the S matrix elements. Firstly, they do not depend on $\mathrm{M}$ and it is not necessary to consider the value of this projection. Secondly, the elements of the parity adapted S

\footnotetext{
${ }^{10}$ The only possible eigenkets for the parity operator are \pm 1
} 
matrix in the helicity representation should be combined according to ${ }^{11}$

$$
\begin{aligned}
& S_{n^{\prime} \Omega^{\prime}, n \Omega}^{E J}=S_{n^{\prime}-\Omega^{\prime}, n-\Omega}^{E J}=\frac{\sqrt{\left(1+\delta_{\Omega^{\prime} 0}\right)\left(1+\delta_{\Omega 0}\right)}}{2}\left[S_{n^{\prime} \Omega^{\prime}, n \Omega}^{E J P=+1}+S_{n^{\prime} \Omega^{\prime}, n \Omega}^{E J P=-1}\right] \\
& S_{n^{\prime} \Omega^{\prime}, n-\Omega}^{E J}=S_{n^{\prime}-\Omega^{\prime}, n \Omega}^{E J}=\frac{(-1)^{J} \sqrt{\left(1+\delta_{\Omega^{\prime} 0}\right)\left(1+\delta_{\Omega 0}\right)}}{2}\left[S_{n^{\prime} \Omega^{\prime}, n \Omega}^{E J P=+1}-S_{n^{\prime} \Omega^{\prime}, n \Omega}^{E J P=-1}\right]
\end{aligned}
$$

before being used for computing reaction observables. In these last equations, $n$ and $n^{\prime}$ represent the quantum numbers not involved in the transformation (that is, $\alpha, v$, $j$ and their products counterparts) and it has been employed the following symmetry of the new $\mathrm{S}$ matrix elements

$$
S_{\alpha^{\prime} v^{\prime} j^{\prime} \Omega^{\prime}, \alpha v j \Omega}^{E J}=S_{\alpha^{\prime} v^{\prime} j^{\prime}-\Omega^{\prime}, \alpha v j-\Omega}^{E J} \quad \text { and } \quad S_{\alpha^{\prime} v^{\prime} j^{\prime}-\Omega^{\prime}, \alpha v j \Omega}^{E J}=S_{\alpha^{\prime} v^{\prime} j^{\prime} \Omega^{\prime}, \alpha v j-\Omega}^{E J}
$$

that stems from the non chirality of reaction (3.35) and, consequently, of the invariance under reflection through the scattering plane 73 . Turning around and merging Eqs. (3.41) and (3.42), we obtain

$$
S_{n^{\prime} \Omega^{\prime}, n \Omega}^{E J P}=\frac{1}{\left[\left(1+\delta_{\Omega 0}\right)\left(1+\delta_{\Omega^{\prime} 0}\right)\right]^{1 / 2}}\left[S_{n^{\prime} \Omega^{\prime}, n \Omega}^{E J}+P(-1)^{J} S_{n^{\prime} \Omega^{\prime}, n-\Omega}^{E J}\right]
$$

Notice that in the above equations $\Omega$ and $\Omega^{\prime}$ values are always defined as positive or zero values.

Next, it will be showed how the cross sections and the reaction probability can be evaluated from the $S_{n^{\prime} \Omega^{\prime}, n \Omega}^{E J}$ matrix elements. We will concentrate on this representation as it will be that employed for the quantum mechanical stereodynamical calculations.

\section{Differential and integral cross sections}

The state-to-state integral cross section for reaction (3.35) can be worked out from the S matrix elements 74

$$
\sigma_{v^{\prime} j^{\prime} \leftarrow v j}=\frac{\pi}{k_{v j}^{2}}(2 j+1)^{-1} \sum_{J}(2 J+1) \sum_{\Omega \Omega^{\prime}}\left|S_{\alpha^{\prime} v^{\prime} j^{\prime} \Omega^{\prime}, \alpha v j \Omega}^{E J}\right|^{2}
$$

\footnotetext{
${ }^{11}$ Again, different phase conventions can give rise to the appearance of different phase factors. The equations presented here are consistent with the convention adopted in the $\mathrm{ABC}$ code 75 , used for calculating some of the scattering matrices that will be lately employed
} 
where $k_{v j}$ is the reactants wavenumber

$$
k_{v j}^{2}=\frac{2 \mu}{\hbar^{2}} E_{\text {coll }}=\frac{2 \mu}{\hbar^{2}}(E-\varepsilon(v, j))
$$

with $E$ representing the total energy, $\varepsilon(v, j)$ the internal energy of the reactants (the energy corresponding to the $(v, j)$ level of the $\mathrm{BC}$ molecule), $E_{\text {coll }}$ the collision energy and $\mu$ the reduced mass of $\mathrm{A}$ and $\mathrm{BC}$.

The differential cross section for the same reaction (in the centre-of-mass system) can be evaluated through the following expression 74 .

$$
\frac{d \sigma_{v^{\prime} j^{\prime} \leftarrow v j}}{d \omega}(\theta)=\frac{1}{4 k_{v j}^{2}}(2 j+1)^{-1} \sum_{\Omega \Omega^{\prime}}\left|\sum_{J}(2 J+1) d_{\Omega^{\prime} \Omega}^{J}(\theta) S_{\alpha^{\prime} v^{\prime} j^{\prime} \Omega^{\prime}, \alpha v j \Omega}^{E J}\right|^{2}
$$

where $d_{\Omega^{\prime} \Omega}^{J}(\theta)$ is a reduced rotation matrix element and $\theta$ is the scattering angle.

Total (summed over all final states) or vibrationally resolved (summed over all the $j^{\prime}$ rotational levels for a certain $v^{\prime}$ state) cross sections can be obtained by direct summation of their state-to-state counterparts defined in Eqs. (3.45) and (3.47). They are often employed in reaction dynamics studies because provide with a general description of the reactivity that allows for making out its main features and because it is not always possible to experimentally resolve the reaction products into different rotational or vibrational levels.

\section{Reaction probability}

The reaction probability as a function of the total angular momentum quantum number $(J)$ is represented as $P(J)$ and given by

$$
P_{v^{\prime} j^{\prime} \leftarrow v j}(J)=\frac{1}{2 \min (J, j)+1} \sum_{\Omega \Omega^{\prime}}\left|S_{\alpha^{\prime} v^{\prime} j^{\prime} \Omega^{\prime}, \alpha v j \Omega}^{E J}\right|^{2}
$$

for state-to-state reactions.

If the products were considered regardless its internal state, the total reaction probability could be worked out

$$
P_{v j}(J)=\sum_{v^{\prime} j^{\prime}} P_{v^{\prime} j^{\prime} \leftarrow v j}(J)=\frac{1}{2 \min (J, j)+1} \sum_{\Omega} \sum_{v^{\prime} j^{\prime} \Omega^{\prime}}\left|S_{\alpha^{\prime} v^{\prime} j^{\prime} \Omega^{\prime}, \alpha v j \Omega}^{E J}\right|^{2}
$$

The importance of $P(J)$ lies in the relation between $J$ and the impact parameter value $b$. On one side, the impact parameter can be evaluated from $l$ (the reactants 
orbital angular momentum quantum number)

$$
b=\frac{\hbar}{\sqrt{2 \mu E_{\mathrm{coll}}}} \sqrt{l(l+1)}
$$

indicating that small (large) values of $l$ correspond to small (large) values of the impact parameter. On the other side, and assuming that the atom A is spinless, the total angular momentum comes from the coupling between the orbital angular momentum and the BC molecule rotational angular momentum. This implies that the accessible values for $J$ are only those contained in the interval

$$
|l-j| \leq J \leq l+j
$$

According to this, the values of $J$ and $l$ will not be very different for reactions where the $\mathrm{BC}$ rotational excitation is small (when $j=0$ they are equal and $P(J)$ coincides with $P(l)$ or, in the classical limit, with the opacity function $P(b)$, the probability of reaction as a function of the impact parameter). This being the case, small (large) values of $J$ can be related as well to small (large) values of the impact parameter and $P(J)$ will approximately (the better the smaller is $j$ ) represent the reactivity dependence on $b$.

\section{Calculation of the scattering matrix elements}

Procedures for carrying out quantum mechanical dynamical calculations for triatomic reactions can be divided into time dependent (TD, see [76, 77, 78] and references therein) and time independent (TI, see 77, 79, 80, and references therein) methods depending on whether they are based on the solution of the time dependent or the time independent nuclear Schödinger equation (Eq. (3.16)). Regardless of the chosen procedure, the aim is to work out the $\mathrm{S}$ matrix elements by studying the scattering properties of the nuclear Hamiltonian $\hat{H}$ (to be consistent with most of the existing literature in scattering theory, it will be represented as $\hat{H}$ instead of $\left.\hat{H}_{\text {nuc }}\right)$ which consists of two terms: one for the relative kinetic energy of the nuclei and another one, given by a PES (adiabatic calculations), for their potential energy.

An introduction to TD and TI methods is presented in the following pages. Only the main features of each technique will be summarized and, in order to simplify the notation, the quantum numbers related to the total angular momentum $(J)$ and the parity $(\mathrm{P})$ will be suppressed. This does not mean that real three dimensional scattering calculations do not consider the symmetry properties of the problem and the corresponding conservation laws but that our schematic presentation of the different 
procedures does not need to consider them.

Time dependent calculations are based on the wavepackets usage and form the simplest group of methods employed to tackle the quantum mechanical study of chemical reactions. They begin by locating a wavepacket $\Phi(\mathbf{x}, t=0)$, that represents the reactants in the state and energies of interest, on the reactants valley. This wavepacket is propagated by solving the time dependent nuclear Schödinger equation

$$
\Phi(\mathbf{x}, t)=\hat{U}(t) \Phi(\mathbf{x}, t=0)=e^{-i \hat{H} t / \hbar} \Phi(\mathbf{x}, t=0)
$$

for a time large enough to describe the studied collisions and, finally, the scattering properties are determined. In practise, the propagation is performed by setting an spatial grid (employing, for instance, reactants mass scaled Jacobi coordinates [79]), representing the wavepacket on the grid and reproducing the effect of the time evolution operator through different standard techniques [76, 81, 82, 83, 84, for wavepackets propagation. Due to the wavepacket spreading with time and to the impossibility of using an infinite grid, the wavepacket has to be absorbed at the grid limits because, otherwise, it would give rise to nonphysical reflections.

Depending on the calculation goal, it may be convenient to switch between different coordinates sets as the wavepacket evolves in time. If the aim of the study is to evaluate total (summed over final states) reaction probabilities, only the flux at the entrance of the products valley is necessary [85] and no coordinates change should be performed. On the contrary, determination of state-to-state quantities (including the elements of the $\mathrm{S}$ matrix) requires to stop the propagation process for changing from reactants to products coordinates in order to avoid the huge computational cost derived from keeping the reactants coordinates during the whole calculation. This coordinates switching represents a crucial problem as it has to be performed in the strong interaction region (where the three atoms are close) and, quite frequently, it can not be efficiently carried out as the wavepacket enters into the products valley without having left the reactants valley. A successful way of overcoming this difficulty is given by the RPD (reactant-product decoupling) method [86, 87], that decouples the reactants and products dynamics. It propagates the initial wavepacket employing reactants coordinates till a plane located at the entrance of the products valley, where it is absorbed through a complex potential. This same potential acts later as a timedependent source that re-emits the wavepacket into the products valley in such a way that the propagation is continued and concluded in terms of products coordinates.

A recent formulation of the quantum mechanical TD methodology, the plane wave packet (PWP) approach developed by Althorpe (see [78, and references therein), 
allows for obtaining the DCS as a function of time, making possible to determine what is the atoms motion during the collision. Calculation of state-to-state reactive differential cross sections can be performed through the PWP approach and also through the real wavepacket approach of Gray and Balint-Kurti [88, 89].

The $\mathrm{S}$ matrix elements as a function of the energy can be extracted from TD stateto-state calculations through the expression [79, 90, 91]

$$
S_{\alpha^{\prime} c^{\prime}, \alpha c}^{E}=-i \hbar \frac{<\Phi^{\prime}\left|G^{+}(E)\right| \Phi>}{<\Phi^{\prime}\left|\phi_{E \alpha^{\prime} c^{\prime}}^{+}><\phi_{E \alpha c}^{-}\right| \Phi>}
$$

that holds for all the energy values for which the denominator is different from zero and where

$$
G^{+}(E)=\lim _{\epsilon \rightarrow 0} \frac{1}{i \hbar} \int_{0}^{\infty} e^{i(E+i \epsilon-\hat{H}) t / \hbar} d t
$$

with $\alpha$ and $\alpha^{\prime}$ representing the atomic rearrangements, $c$ and $c^{\prime}$ the rest of quantum numbers necessary to specify the channel, $\Phi$ and $\Phi^{\prime}$ the initial and final wavepackets (that is, the wavepackets describing the reactants (products) before (after) the beginning (end) of the collision) and $\phi_{E \alpha^{\prime} c^{\prime}}^{+}$and $\phi_{E \alpha c}^{-}$outgoing and incoming asymptotic wavefunctions (formed by the product between a plane wave representing the approaching (incoming) or recoiling (outgoing) movement of the particles involved in the corresponding channel and a function describing their internal state).

TI calculations focus on the time independent nuclear Schödinger equation

$$
\hat{H} \psi_{E}(\mathbf{x})=E \psi_{E}(\mathbf{x})
$$

where $\hat{H}$ represents the nuclear Hamiltonian operator, $E$ the total energy for which the collision is studied and $\psi_{E}(\mathbf{x})$ the corresponding stationary scattering state. There is a variety of methods which employ this equation and, among them, the coupled channel hyperspherical approach and the variational methods have been the most widely employed for reactive scattering calculations and will be now sketched.

The coordinates problem (coordinates suitable for describing the reactants are not useful for products and vice versa) is also present in TI calculations. However, the employment of hyperspherical coordinates (they were initially applied to nuclear physics problems [92, 93, 94] and then applied to chemical reactions (see [79, 80, and references therein)) makes unnecessary to halt the calculations for switching between sets of coordinates suitable for the different atomic arrangements involved in a reaction. These coordinates display three interesting features:

- They naturally transform into well behaved coordinates for reactants and prod- 
ucts in the corresponding asymptotic regions.

- Unbounded movement is related to only one degree of freedom, the hyperradius $(\rho)$, which represents a measure of the distance between the particles involved in the collision. The rest of hyperspherical coordinates will be termed as "bounded" because correspond to bounded motions.

- They provide with simple (compared to other coordinates) expressions for the kinetic energy operator and the volume element.

which make them very convenient for reactive scattering studies and justify their employment ${ }^{12}$. The coupled channel hyperspherical approach (see [79, 80] for more details and references) makes use of these coordinates for calculating the $\mathrm{S}$ matrix elements and can be outlined as

- The wavefunction $\psi_{E}$ is represented through a series

$$
\psi_{E}=\rho^{-1 / 2} \sum_{n} d_{n}(\rho) \phi_{n}(\nu)
$$

where the expansion coefficients $d_{n}$ depend on the hyperradius and the functions $\phi_{n}$ are solutions of Eq. (3.55) at a fixed value of the hyperradius (they depend on the bounded hyperspherical coordinates (represented generically as $\nu$ ).

- Substitution of Eq. (3.56) into the time independent Schödinger equation (Eq. (3.55)) provides with a system of coupled ordinary differential equations for the $d_{n}$ coefficients that, when solved, completely determines $\psi_{E}$. The integration of the system is performed from the repulsive region of the PES (small values of $\rho$ ), where the $d_{n}$ coefficients vanish, to a large value of $\rho$ corresponding to points on the reactants and products valleys where the interaction between the atom and the diatomic molecule can be neglected.

- The elements of the S matrix can be worked out by applying an asymptotic analysis to the scattering wavefunction $\psi_{E}$. Assuming that (i) the PES controlling the nuclear dynamics fits into the conditions regarding short and long-distance behaviour presented in Sec. 3.1 and (ii) that the reactants state corresponds to the channel labelled by the quantum numbers $\alpha$ (atomic arrangement) and $c$ (as before, it includes any other quantum number necessary for the complete

\footnotetext{
${ }^{12}$ In view of their properties, the reader could inquire why these coordinates were not mentioned during the former presentation of TD methods. The reason is that, at the moment, there is no efficient procedures to incorporate them into wavepacket calculations and, at the same time, convenient alternatives exist
} 
specification of the channel and, in particular, those corresponding to the internal state of the diatomic molecule), the asymptotic behaviour of $\psi_{E}$ will be given by the following condition

$$
\psi_{E} \sim \phi_{E \alpha c}^{-}-\sum_{c^{\prime}} \phi_{E \alpha c^{\prime}}^{+} S_{\alpha c^{\prime}, \alpha c}^{E}
$$

in the reactants valley and by

$$
\psi_{E} \sim-\sum_{c^{\prime \prime}} \phi_{E \alpha^{\prime} c^{\prime \prime}}^{+} S_{\alpha^{\prime} c^{\prime \prime}, \alpha c}^{E}
$$

in the products valley. The sums run for all the possible energetically accessible reactants $\left(c^{\prime}\right)$ and products $\left(c^{\prime \prime}\right)$ states and $S_{\alpha c^{\prime}, \alpha c}^{E}\left(S_{\alpha^{\prime} c^{\prime \prime}, \alpha c}^{E}\right)$ holds for the inelastic and elastic (reactive) elements of the $\mathrm{S}$ matrix. The interpretation of the asymptotic conditions is simple; in the reactants valley $\psi_{E}$ must coincide with the sum of an incoming wavefunction $\phi_{E \alpha c}^{-}$that represents the approaching reactants and outgoing wavefunctions $\phi_{E \alpha c^{\prime}}^{+}$representing the inelastically and elastically scattered reactants. Equally, in the products valley, $\psi_{E}$ can be decomposed as the sum of different outgoing wavefunctions $\phi_{E \alpha^{\prime} c^{\prime \prime}}^{+}$that correspond for the reaction products in all the opened channels. According to this, the $\mathrm{S}$ matrix elements will be given by the projection of $\psi_{E}$ over the different asymptotic wavefunctions involved in Eqs. (3.57) and (3.58).

- In practise, the calculation is slightly more complicated. Considering slices of the PES at fixed values of $\rho$, their profiles turn out to be completely different depending on whether $\rho$ is large or small. In consequence, it is not possible to use a single set of functions $\phi_{n}$ for the whole range of $\rho$ values but, instead, these functions will have to depend parametrically on the hyperradius. This determines that the integration of the equations system obtained by substituting (3.56) into (3.55) has to be performed through a sector-by-sector procedure where the potential is divided in many sectors along $\rho$, the functions $\phi_{n}$ and the coefficients $d_{n}$ are determined at the center of each sector and the global continuity of $\psi_{E}$ is ensured by imposing boundary conditions at the sector limits.

The two S matrix evaluation procedures presented so far involve a propagation with respect to time (TD wavepackets) or to the hyperradius (TI coupled channel hyperspherical) and, in both cases, the efficiency of the calculation was directly related to the coordinates employed for the propagation. The last group of methods that will be presented, the variational methods [95], do not include any propagation 
and, therefore, it is not necessary to rack one's brain looking for the right coordinates. Variational methods are TI and reduce the scattering calculation to a linear algebra problem (that involves basis, matrices elements and matrices operations) by expressing the $\mathrm{S}$ matrix elements as a functiona ${ }^{13} S_{\alpha^{\prime} c^{\prime}, \alpha c}\left[\widetilde{\psi}_{E}\right]$ with the following characteristics

- The trial functions $\widetilde{\psi}_{E}$ must fulfill asymptotic conditions similar to Eqs. (3.57) and (3.58).

- When $\widetilde{\psi}_{E}=\psi_{E}$ (the solution of the time independent Schödinger equation), the value of the functional coincides with the $\mathrm{S}$ matrix element $S_{\alpha^{\prime} c^{\prime}, \alpha c}$.

- The functional is stationary with respect to variations $\delta \psi_{E}$ around $\psi_{E}$ or, in other words

$$
S_{\alpha^{\prime} c^{\prime}, \alpha c}\left[\psi_{E}+\delta \psi_{E}\right]=S_{\alpha^{\prime} c^{\prime}, \alpha c}+O\left(\left(\delta \psi_{E}\right)^{2}\right)
$$

These two features determine that $S_{\alpha^{\prime} c^{\prime}, \alpha c}$ can be worked out as if we were solving an elementary variational problem: (i) the trial function $\widetilde{\psi}_{E}$ is taken as a linear combination of the functions of a certain basis (they use to be vibrational-rotational states of reactants and products) with arbitrary coefficients $c_{i}$, (ii) $\widetilde{\psi}_{E}$ is substituted in the functional and (iii) the coefficients of the expansion are now fixed by imposing that

$$
\frac{\partial S_{\alpha^{\prime} c^{\prime}, \alpha c}\left[\widetilde{\psi}_{E}\right]}{\partial c_{i}}=0 \quad \forall i
$$

The trial function for these coefficients is the best possible one for the chosen basis and, when substituted in the functional, it leads to an approximated value for $S_{\alpha^{\prime} c^{\prime}, \alpha c}$.

Both TD and TI calculations present advantages and disadvantages that can be summarized as follows

- Implementation of TD methods is closer to their theoretical formulation than for their TI counterparts. This makes TD calculations easiest to use and understand.

- TD methods are not feasible at very low collisions energies due to (i) the necessity of large propagation times and to (ii) inaccuracies related to the employment of absorbing potentials at the grid limits.

- While TD methods provide with one column (that corresponding to the reactants channel) of the scattering matrix for all the energies included in the

\footnotetext{
${ }^{13}$ Roughly speaking, a functional is a function of functions, that is, a function whose values depend on other functions and not on real or complex independent variables. For instance, the classical action is a functional of the Lagrangian 68
} 
wavepacket, TI calculations allow for evaluating the whole S matrix for only one energy.

- The computational effort necessary for carrying out the calculations increases faster for TI than for TD methods. This makes unfeasible the employment of TI calculations for high dimensionality problems (for instance, collisions involving four atoms).

- TD calculations make possible the generation of "films" describing the collision evolution as time elapses [96]. This films represent a powerful interpretation tool.

The selection of one method or the other depends on the studied reaction, on the features of the experiment that is going to be simulated and on the available computational resources. 


\section{Reaction control and mechanism analysis through the reactants polarisation}

In the two preceding Chapters it has been discussed how to mathematically describe the angular momentum polarisation and to perform classical and quantum mechanical dynamical calculations for atom-diatom collisions. Using them as starting point, this Chapter will present a set of theoretical methods to analise the influence of the reactants rotational angular momentum $(\mathbf{j})$ polarisation on atom-diatom collisions. In particular, it will be showed that the $\mathbf{j}$ polarisation is a suitable tool for (i) probing the mechanism of the process and for (ii) controlling the reaction probability and products state distribution. All what is needed to perform this kind of study is the outcome of the dynamical calculation (the quasiclassical trajectories or the scattering matrix) and, although the exposition will concentrate on reactive collisions, the methods are also suitable for application on inelastic or elastic processes.

The properties of chemical reactions can be divided into two categories: intrinsic and extrinsic properties (Sec. 4.1). The former represent the inherent properties of the reaction (they can not be modified) while the second describe the experimental setup (they can be externally controlled). In particular, it can be distinguished between the intrinsic and the extrinsic reactants polarisations (Sec. 4.2). The intrinsic one is the $\mathbf{j}$ polarisation that gives rise to reaction and it will be the basis for our analysis of the mechanism. In turn, the extrinsic polarisation represents the actual $\mathbf{j}$ polarisation when an experiment is carried out. The consideration of different extrinsic polarisation schemes will allow for controlling the reaction output.

Both polarisations can be described by means of the corresponding polarisation moments (Chapter 2) whose values contain information about the alignment or orientation of $\mathbf{j}$ along defined directions in the space. A presentation of the different kinds of intrinsic moments, their characteristics and the details of their calculation is included in Sec. 4.3 .

The reaction control can be studied by considering that the output of an experiment 
depends both on the intrinsic properties of the reaction and on the characteristics of the experiment (the extrinsic properties) that is being performed. Sec. 4.4 shows that the reaction cross sections can be expressed as a function of the intrinsic and the extrinsic polarisations and provides with explicit expressions for that dependence. These expressions allow for a quantitative and qualitative analysis of the control reaction that can be achieved by means of the $\mathbf{j}$ polarisation.

If what one wants is insight (that is, a rationalisation of reaction mechanism), then looking at the spatial distributions of molecular axis and rotational angular momentum is likely more useful than analysing a long list of polarisation parameters. This can be achieved by means of the stereodynamical portraits (Sec. 4.5) that are three dimensional plots representing the $\mathbf{j}$ and $\mathbf{r}$ (molecular axis of the reactive diatomic molecule) distributions related to any intrinsic or extrinsic polarisation.

Sec. 4.6 describes a possible experiment consisting on the reactant diatomic alignment along a chosen direction followed by the monitoring of the reaction probability (cross sections) for that extrinsic preparation. This experiment will fulfill a double role: it represents a feasible procedure to check the validity of the methods presented in this Chapter and, due to its simple interpretation, it provides with a detailed and intuitive panorama of the steric requirements of the reaction.

To end with, Sec. 4.7 shows results that illustrate (i) how the theory can be used and (ii) the kind of information that can be obtained from the proposed experiment. The examples employ the $\mathrm{H}+\mathrm{D}_{2}$ benchmark reaction and focus on three aspects of the reaction: the mechanism analysis, the control possibilities and the evaluation of the control limits.

The electronic and nuclear spins will be ignored, which means that the contain of the Chapter will be valid only when the BC reactant is a closed shell molecule not susceptible to fast hyperfine depolarisation [29, 31, 38.

Unless otherwise stated, all the equations, concepts and ideas presented will hold regardless of the usage of classical or quantum mechanics. Any difference between both treatments will be explicitly indicated and discussed.

\subsection{Intrinsic and extrinsic properties of chemical reactions}

The methods and results included in this Chapter are introduced on the basis of the distinction between intrinsic and extrinsic properties of chemical reactions. That differentiation stems from the recognition that, in a sense, chemical reactions exist 
independently from the actual and specific preparation of reacting molecules.

The intrinsic properties are those which describe the reactive process itself, do not depend on any external circumstance and cannot be controlled. In contrast, the extrinsic properties are always associated with the specific circumstances under which the reaction takes place. They represent the experimental setup, depend on external factors and, at least in principle, can be controlled just by changing the experimental arrangement. Summarizing, we could say that the intrinsic properties represent "what the reaction wants" while the extrinsic properties represent "what the reaction gets from the experiment".

For instance, the scattering matrix obtained in quantum mechanical reactive scattering calculations is an intrinsic property. As it was discussed in the former Chapter, its elements represent transition probability amplitudes that tie reactants to products states. Once that its elements have been worked out they can be employed to work out reaction observables such as the integral and the differential cross sections or the polarisation moments that will be defined in Sec. 4.3. However, before of evaluating these quantities it is necessary to fix the value of certain extrinsic properties such as the reactants and products internal states, the scattering angle or the reactants polarisation.

\subsection{Intrinsic and extrinsic reactants polarisations}

Within the different reaction properties, we will concentrate on the intrinsic and the extrinsic polarisations of the reactants. The former is the $\mathbf{j}$ (rotational angular momentum of the diatomic reactant molecule) polarisation that leads to formation of the products. It contains the directional preferences of the reaction and, in consequence, it will be used for the analysis of the reaction mechanism. In turn, the extrinsic polarisation corresponds to the actual preparation of the reactants polarisation when an experiment takes place and (in contrast with its intrinsic counterpart) it can be externally modified and used to control the output of the reaction. As long as the reactants polarisation is not extrinsically prepared, the target molecule is completely unpolarised (see Sec. 2.3.3). This means that the rotational angular momentum and the internuclear axis of the reactant molecule $(\mathbf{r})$ are isotropically distributed and that the results obtained from the experiment represent an average over all the possible polarisations of the reactants. Of course, the experiments could be carried out with different extrinsic polarisations and it would be found that they influence the outcome of the experiment (favouring or hindering it). As it will be discussed in Sec. 4.4.4, the outcome of the reaction always increases when the extrinsic polarisation matches 
the intrinsic one. In some cases, this increment can be the maximum possible and, in other, be only an improvement with respect to the unpolarised reactants collisions. In any case, the intrinsic polarisation reflects the reaction directional preferences ("what the reaction wants") and, in consequence, it indicates what to do for increasing (or decreasing) the reaction yield.

The intrinsic moments can be determined either theoretically or experimentally. Next Section will include the procedure to work out these moments from QCT or quantum mechanical calculations. To experimentally determine the intrinsic polarisation of the reactants [97] it would be necessary to use a variety of extrinsic polarisations which can be obtained by changing the experimental "geometries" with respect to an space fixed frame (LAB frame); that is, the direction of the electric field, or the polarisation vectors corresponding to the lasers used for the preparation of the reagents, etc. From the analysis of the experimental results obtained under different extrinsic preparations one can derive the intrinsic polarisation.

\subsection{Intrinsic polarisation moments}

This Section introduces the different intrinsic polarisation moments, their properties and the procedure to evaluate them. The calculations always begin by working out the intrinsic polarisation moments for state-to-state reactions resolved with regard to scattering angle. These polarisation moments are termed as state-to-state polarisation dependent differential cross sections (state-to-state PDDCSs) and allow for a very detailed stereodynamical analysis of the reaction. The state-to-state PDDCSs serves as well as starting point for the calculation of the intrinsic moments corresponding to less detailed processes:

- the state-to-state polarisation parameters (state-to-state PPs) will be obtained by integration over the scattering angle and

- differential and integral polarisation moments averaged over product states (total PDDCSs and PPs) can be worked out from their state-to-state counterparts.

The structure of this Section reflects the order in which calculations are done. It begins with the definition of state-to-state PDDCSs, goes through the definitions of the other moments and finish with a subsection including the explicit procedure for evaluating the PDDCSs. The reason for presenting the PDDCSs calculation at the end instead of immediately after their introduction is to show the relation between the different intrinsic moments in the clearest way, that is, without interferences in the sequence that goes from more detailed to less detailed moments. 


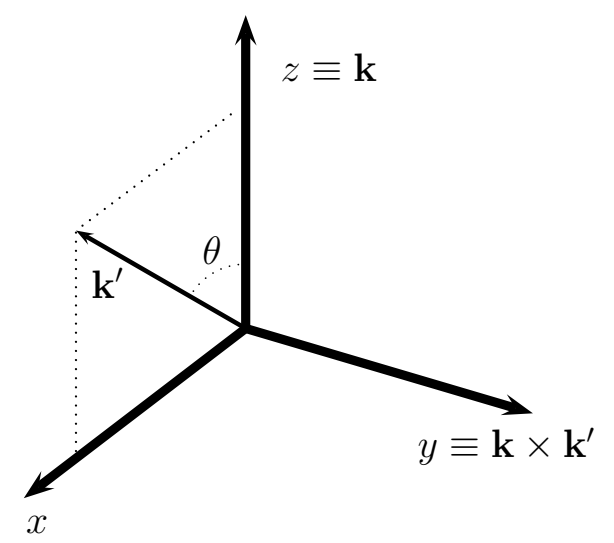

Fig. 4.1.: Centre-of-mass reference frame. The reactants approach direction (k) is parallel to the $z$ axis, $\mathbf{k}^{\prime}$ is the products recoil direction and $\theta$ represents the scattering angle.

Except for (i) the calculation procedure, (ii) the limitation of the number of moments in the quantum mechanical case and (iii) the upper and lower limits for their values (see App. B), the PDDCSs (PPs) have the same characteristics and meaning regardless of their classical or quantum mechanical origin.

The directional information contained in the intrinsic moments will be referred to the $x y z$ centre-of-mass reference frame ${ }^{11}$ (sketched in Fig. 4.1) whose $z$ axis coincides with $\mathbf{k}$ (the reactants relative velocity), and whose $y$ axis coincides with $\mathbf{k} \times \mathbf{k}^{\prime}\left(\mathbf{k}^{\prime}\right.$ is the products relative velocity). This implies that the scattering plane is $x z$ and that $\mathbf{k}^{\prime}$ lies on the $x \geq 0$ half of the scattering plane. Operators, moments and vectors defined in this system will be denoted with low case letters: $q$ will be used to label the components of the polarisation moments and $\theta_{j}$ and $\phi_{j}\left(\theta_{r}\right.$ and $\left.\phi_{r}\right)$ to represent the polar and azimuthal angles that describe the direction of $\mathbf{j}(\mathbf{r})$ (remember that the former is the rotational angular momentum of the reactant diatomic molecule while the second is its internuclear axis).

\subsubsection{State-to-state PDDCSs}

So far, an state-to-state atom-diatom reaction has been written as

$$
\mathrm{A}+\mathrm{BC}(v, j) \stackrel{E_{\mathrm{coll}}, \theta}{\longrightarrow} \mathrm{AB}\left(v^{\prime}, j^{\prime}\right)+\mathrm{C}
$$

\footnotetext{
${ }^{1}$ The $x y z$ frame will be named as well "scattering frame"
} 
where $v$ and $j\left(v^{\prime}\right.$ and $\left.j^{\prime}\right)$ are the vibrational and rotational quantum numbers which specify the internal state of the reactant (product) diatomic molecule, $E_{\text {coll }}$ is the collision energy and $\theta$ is the scattering angle. This expression only takes into consideration the internal states of the molecules but not their polarisation. In order to incorporate such polarisation into the study, it is convenient to think about an atom-diatom chemical reaction as being described by the process [24, 98 ,

$$
\mathrm{A}+\mathrm{BC}(v, j, k, q) \stackrel{E_{\mathrm{coll}}, \theta}{\longrightarrow} \mathrm{AB}\left(v^{\prime}, j^{\prime}, k^{\prime}, q^{\prime}\right)+\mathrm{C}
$$

where the new labels ( $k, q$ and their primed counterparts) are associated with the moments (see Chapter 2 and references therein) that characterise the $\mathrm{BC}$ and $\mathrm{AB}$ rotational angular momentum $\left(\mathbf{j}\right.$ and $\left.\mathbf{j}^{\prime}\right)$ polarisations.

In this and the two following Chapters our interest will lie in reactants rather than products polarisation and, therefore, the products-polarisation labels will be fixed at $k^{\prime}=q^{\prime}=0$ (this means that the products are considered regardless of their polarisation state) and not explicitly mentioned. The state-to-state polarisation dependent differential cross sections (state-to-state PDDCSs) 25] are the moments which characterize the intrinsic $\mathbf{j}$ polarisation for the resulting process

$$
\mathrm{A}+\mathrm{BC}(v, j, k, q) \stackrel{E_{\mathrm{coll}}, \theta}{\longrightarrow} \mathrm{AB}\left(v^{\prime}, j^{\prime}\right)+\mathrm{C}
$$

They represent the $\mathbf{j}$ alignment ( $k$ even) or orientation ( $k$ odd) along defined directions in the space when the reaction (4.3) takes place (see Sec. 2.4.2 for details), describe the interplay between molecular polarisation and reactivity at any given scattering angle (the $\mathbf{j}-\mathbf{k}-\mathbf{k}^{\prime}$ three-vector correlations) and quantify

- the probability that the $\mathrm{AB}\left(v^{\prime}, j^{\prime}\right)$ product is found to recoil along the final relative velocity direction $\mathbf{k}^{\prime} \ldots$

- ...if the $\mathrm{A}$ atom approaches the $\mathrm{BC}(v, j)$ molecule along the relative velocity direction $\mathbf{k} \ldots$

- ...with $\mathrm{BC}(v, j)$ having the particular kind of polarisation associated with the PDDCS labels $k$ (the PDDCS rank) and $q$ (the PDDCS component).

To gain an insight into the PDDCSs definition, it is convenient to remember that the polarisation moments were introduced as the coefficients of an expansion (Chapter 2). In this case, the expanded functions are [25, 32]

- Classical mechanics: the probability density function $P_{r}\left(\theta, \theta_{j}, \phi_{j}\right)$, whose values give the probability of observing reactive scattering from a given reactants rovi- 
brational state into a given products rovibrational state and at scattering angle $\theta$ when the initial direction of the $\mathrm{BC}$ reactant rotational angular momentum is the one associated with the spherical angles $\theta_{j}$ and $\phi_{j}$ (all the angles referred to the centre-of-mass reference frame).

- Quantum mechanics: the matrix elements $\left\langle j \Omega_{1}|\hat{\rho}(\cos (\theta))| j \Omega_{2}\right\rangle$, where $\hat{\rho}(\cos (\theta))$ is the rotational-space density operator of the reactants in the process (4.3). This operator represents the state of the reacting BC molecules in the space expanded by the $|j \Omega\rangle$ basis, where $j$ is the rotational angular momentum quantum number and $\Omega$ is the helicity (the projection of $\mathbf{j}$ over $\mathbf{k}$ ). The density matrix elements take into account the coherences between the different values of the helicity.

and the explicit form of the expansions (in terms of the complex PDDCSs represented by $\left.S_{q}^{(k)}(\theta)\right)$ is given by 25,32 .

$$
P_{r}\left(\theta, \theta_{j}, \phi_{j}\right)=\sum_{k=0}^{\infty} \sum_{q=-k}^{k} \frac{2 k+1}{4 \pi} S_{q}^{(k)}(\theta) C_{k q}^{*}\left(\theta_{j}, \phi_{j}\right)
$$

(compare with Eq. (2.2)) and

$$
\left\langle j \Omega_{1}|\hat{\rho}(\cos (\theta))| j \Omega_{2}\right\rangle=\sum_{k=0}^{2 j} \sum_{q=-k}^{k} \frac{2 k+1}{2 j+1} S_{q}^{(k)}(\theta)\left\langle j \Omega_{1} k q \mid j \Omega_{2}\right\rangle
$$

(compare with Eq. (2.42)).

Following Sec. 2.4, the PDDCSs can be represented as complex or real moments. While the first representation is convenient for calculations (because the complex PDDCSs transform under rotation as the angular momentum eigenstates (see App. A)), the second is convenient for interpretation purposes because it allows one to consider molecular polarisations in terms of Cartesian directions. Complex PDDCSs are represented by $S_{ \pm q}^{(k)}(\theta)$ or $\operatorname{PDDCS}(k, \pm q)$ and real PDDCSs by $S_{q \pm}^{\{k\}}(\theta)$ or $\operatorname{PDDCS}\{k, q \pm\}$. The transformation between both sets of moments is given by Eq. (2.54) or (2.55).

All the reactions that will be studied are non-chiral reactions, that is, reactions which are symmetric with respect to the scattering $(x z)$ plane [25]. In other words, this means that the atomic ensemble which gives rise to reaction can not distinguish between "up" and "down" with respect to the scattering plane [33. As it was discussed in Chapter 2, this symmetry will restrict the values that the PDDCSs can take 
because, on top of the condition

$$
S_{q}^{(k)}(\theta)=(-1)^{q} S_{-q}^{(k) *}(\theta) \quad \forall k, q
$$

(general for all the polarisation moments. See Eq. (2.5) (classical mechanics) and (2.44) (quantum mechanics)) the complex PDDCSs must fulfill Eq. (2.50)

$$
S_{q}^{(k)}(\theta)=(-1)^{k+q} S_{-q}^{(k)}(\theta) \quad \forall k, q
$$

derived from the invariance through $x z$ plane reflections. It follows from these two conditions that the complex PDDCSs with $\mathrm{k}$ even (odd) are real (pure imaginary) and that those with $k$ odd and $q=0$ vanish. As for the real PDDCSs, it implies that the only nonvanishing real PDDCSs are

$$
\begin{aligned}
k \text { even: } & S_{0}^{\{k\}}(\theta) \text { and } S_{q+}^{\{k\}}(\theta), \quad 1 \leq q \leq k \\
k \text { odd: } & S_{q-}^{\{k\}}(\theta), \quad 1 \leq q \leq k .
\end{aligned}
$$

As it will be shown in the next Section, the $S_{0}^{(0)}(\theta)$ moment (whose values represent the population of the reacting $\mathrm{BC}$ molecules state) coincides with the angular distribution $^{2}$ when the reactants are initially unpolarised. The angular distribution is, in general, a non constant function whose values depend on the scattering angle. This involves that (see Sec. 2.4.3): (i) the intrinsic polarisation corresponding to different scattering angles can not be directly compared by means of the PDDCSs because a certain moment can take the same value at two different angles but representing different polarisation intensities and, (ii) in order to perform that comparison or to set in absolute terms the intensity of a polarisation it is necessary to introduce the real renormalised PDDCSs

$$
\frac{S_{q}^{\{k\}}}{S_{0}^{\{0\}}} \quad \forall k, q
$$

(the equation is equally valid for the complex PDDCSs) that refer the moments to states of population one (and adopts the corresponding scale).

\subsubsection{State-to-state PPs}

By correlating reactant polarisation to scattering angle in a state-to-state fashion, state-to-state PDDCSs provide a very detailed description of polarisation effects in reaction dynamics.

\footnotetext{
${ }^{2}$ The probability of reaction as a function of the scattering angle 1
} 
But, is that level of detail always desired? The answer is no. In fact, state-to-state PDDCSs rarely offer a convenient starting point for stereodynamical analyses. Good starting points are offered by less detailed quantities, which allow for a more global view of the problem of interest and for an easier identification of the dominant effects.

There are two natural ways of reducing the level of detail of state-to-state PDDCSs: integration over the scattering angle and averaging over product states. This Section deals with the first.

The polarisation parameters (PPs) are the counterparts of the PDDCSs when the integration over the scatering angle is performed and represent the intrinsic polarisation of the process

$$
\mathrm{A}+\mathrm{BC}(v, j, k, q) \stackrel{E_{\text {coll }}}{\longrightarrow} \mathrm{AB}\left(v^{\prime}, j^{\prime}\right)+\mathrm{C}
$$

They inform about the $\mathbf{j}$ polarisation when this reaction takes place, describe the interplay between molecular polarisation and reactivity regardless of the scattering angle (the $\mathbf{j}$-k two-vector correlation) and quantify:

- the probability that the $\mathrm{AB}\left(v^{\prime}, j^{\prime}\right)$ product is formed...

- ...when the $\mathrm{BC}(v, j)$ reactant has the particular kind of polarisation associated with the PP labels $k$ (the PP rank) and $q$ (the PP component).

The nature of the functions whose expansion coefficients are the PPs and the explicit expression for the corresponding expansions could be easily deduced [25] from their PDDCSs counterparts (Eqs. (4.4) and (4.5)) just by considering (i) the integration over the scattering angle and (ii) the subsequent substitution of the PDDCSs by the PPs.

Complex PPs are represented by $s_{ \pm q}^{(k)}$ or $\operatorname{PP}(k, \pm q)$, and their values can be worked out from complex PDDCSs by use of the formula [25]

$$
s_{q}^{(k)}=\int_{-1}^{1} S_{q}^{(k)}(\theta) d(\cos \theta) .
$$

In particular, the value of the $s_{0}^{(0)}$ moment is calculated integrating the angular distribution $\left(S_{0}^{(0)}(\theta)\right)$ and, therefore, it always will be equal to one. In consequence, it is not necessary to renormalise the PPs before to compare the intrinsic polarisations for different reactants and products states in Eq. (4.10). The application of the combination scheme given by Eq. (2.54) or $(2.55)$ to the $s_{ \pm q}^{(k)}$ moments allows for the definition of the real PPs. They are represented as $s_{q \pm}^{\{k\}}$ or $\operatorname{PP}\{k, q \pm\}$ and have the same directional meaning as the real PDDCSs. 


\subsubsection{Total PDDCSs}

The second way of reducing the level of detail of state-to-state PDDCSs consists of averaging over product states, a procedure that leads to the total PDDCSs.

The average over final states uses the state-to-state integral cross sections as statistical weights in order to account for the different probabilities of the various stateto-state processes:

$$
S_{q, \mathrm{tot}}^{(k)}(\theta)=\frac{\sum_{v^{\prime}, j^{\prime}} \sigma\left(v^{\prime}, j^{\prime}\right) S_{q}^{(k)}(\theta)}{\sum_{v^{\prime}, j^{\prime}} \sigma\left(v^{\prime}, j^{\prime}\right)}
$$

where the $\sigma\left(v^{\prime}, j^{\prime}\right)$ are the state-to-state integral cross sections, and $S_{q \text {,tot }}^{(k)}(\theta)$ the total PDDCSs. Note that, although our notation does not make it explicit, the state-tostate PDDCSs, $S_{q}^{(k)}(\theta)$, do depend on $v^{\prime}$ and $j^{\prime}$. If the averaging is only over the $j^{\prime}$ states corresponding to a given vibrational manifold, then the resulting PDCCS are $S_{q, v^{\prime}}^{(k)}(\theta)$.

Note also that, just like their state-to-state counterparts, total PDDCSs may or may not require renormalisation [that is, division by $S_{0, \text { tot }}^{(0)}(\theta)$ ].

\subsubsection{Total PPs}

When combined, the integration and averaging procedures described in the last two pages lead to total PPs. These quantities, related to the state-to-state PPs by

$$
s_{q, \text { tot }}^{(k)}=\frac{\sum_{v^{\prime}, j^{\prime}} \sigma\left(v^{\prime}, j^{\prime}\right) s_{q}^{(k)}}{\sum_{v^{\prime}, j^{\prime}} \sigma\left(v^{\prime}, j^{\prime}\right)}
$$

provide the best starting points for stereodynamical analyses as they make possible to obtain the most general perspective of the reaction stereodynamics.

\subsubsection{Calculation of the state-to-state PDDCSs}

The state-to-state PDDCSs can be worked out from quasiclassical trajectories and from quantum-mechanical methods.

\section{Quasiclassical trajectories evaluation of the PDDCSs}

The quasiclassical trajectories (QCT) calculations (see Sec. 3.3) are carried out using an uniform distribution of the reactants rotational angular momentum or internuclear axis, without any polarization bias. Of course, each individual trajectory is associated with well defined initial and final polarization states, but the ensemble of 
trajectories spans an uniform, isotropic distribution of directions. It is the analysis of the subset of trajectories tying each initial rovibrational state to each final rovibrational state that allows for the PDDCSs calculation, that is, for the analysis of the $\mathbf{j}$ polarisation when the reaction is giving rise to a particular final state at a given scattering angle.

The procedure for evaluating the PDDCSs can be summarized as follows [25, 41]. Each particular combination of reactant and product rovibrational states is considered in turn, along with the corresponding subset of trajectories. This subset contains $N_{v j v^{\prime} j^{\prime}}$ trajectories, labelled by the index $i$. The values of the scattering angle $\theta^{(i)}$ and the polar and azimuthal angles that define the direction of $\mathbf{j}, \theta_{j}^{(i)}$ and $\phi_{j}^{(i)}$, are determined for each of the $N_{v j v^{\prime} j^{\prime}}$ trajectories. This information is used to calculate the values of all the modified spherical harmonics of interest for each trajectory (classically, the polarisation moments were expressed as the expectation value of modified spherical harmonics (Eq. (2.4))), and then the trajectory-specific values are averaged over the $N_{v j v^{\prime} v j^{\prime}}$ reactive trajectories.

The PDDCSs can be written as a series of modified spherical harmonics [25, 41]

$$
S_{q}^{(k)}(\theta)=\frac{1}{2} \sum_{k_{1} \geq|q|}^{\infty}\left(2 k_{1}+1\right) s_{k q}^{k_{1}} C_{k_{1}-q}(\theta, 0)
$$

where the coefficients $s_{k q}^{k_{1}}$ are given by

$$
\begin{aligned}
s_{k q}^{k_{1}} & =\left\langle C_{k_{1}-q}(\theta, 0) C_{k q}\left(\theta_{j}, \phi_{j}\right)\right\rangle \\
& =\frac{1}{N_{v j v^{\prime} j^{\prime}}} \sum_{i=1}^{N_{v j v^{\prime} j^{\prime}}} C_{k_{1}-q}\left(\theta^{(i)}, 0\right) C_{k q}\left(\theta_{j}^{(i)}, \phi_{j}^{(i)}\right)
\end{aligned}
$$

and the brackets indicate the averaging over the whole set of trajectories associated with the chosen rovibrational states of reactants and products.

The PPs can be evaluated by integrating over the scattering angle (Eq. (4.11) ) or, equivalently, as [25, 41]

$$
s_{q}^{(k)}=\left\langle C_{k q}\left(\theta_{j}, \phi_{j}\right)\right\rangle=\frac{1}{N_{v j v^{\prime} j^{\prime}}} \sum_{i=1}^{N_{v j v^{\prime} j^{\prime}}} C_{k q}\left(\theta_{j}^{(i)}, \phi_{j}^{(i)}\right)
$$

\section{Quantum mechanical evaluation of the PDDCSs}

The starting point of the derivation of the quantum mechanical expression for the reactants intrinsic PDDCSs is the scattering matrix in the helicity representation 
(Sec. 3.4 and ref. 73 ), the one most naturally adapted for the description of the $\mathbf{j}-\mathbf{k}-\mathbf{k}^{\prime}$ vectors correlation 24,98 .

Complete specification of a particular element of this matrix requires a notation such as $S_{a^{\prime} v^{\prime} j^{\prime} \Omega^{\prime}, a v j \Omega}^{J}$, with the various indices indicating the total energy $(E)$, total angular momentum $(J)$ and arrangement, vibrational, rotational and helicity quantum numbers for reactants $\left(a, v, j\right.$ and $\Omega$ ) and products $\left(a^{\prime}, v^{\prime}, j^{\prime}\right.$ and $\left.\Omega^{\prime}\right)$. As the following equations only require implicit use of the energy, arrangement and vibrational labels, the notation will be simplified to $S_{j^{\prime} \Omega^{\prime}, j \Omega}^{J}$.

Once that the S matrix elements are known, the following expression allows for evaluating the PDDCSs [25]:

$$
\left[S_{q}^{(k)}(\theta)\right]^{*}=\sum_{\Omega^{\prime} \Omega_{1} \Omega_{2}} \bar{f}_{\Omega^{\prime} \Omega_{1}}(\theta) \bar{f}_{\Omega^{\prime} \Omega_{2}}^{*}(\theta)\left\langle j \Omega_{1}, k q \mid j \Omega_{2}\right\rangle
$$

where the $\bar{f}$ functions denote "scaled" scattering amplitudes [74] given by

$$
\bar{f}_{\Omega^{\prime} \Omega}(\theta)=\frac{\sum_{J}(2 J+1) d_{\Omega^{\prime} \Omega}^{J}(\theta) S_{j^{\prime} \Omega^{\prime}, j \Omega}^{J}}{\left[2 \sum_{J \Omega^{\prime} \Omega}(2 J+1)\left|S_{j^{\prime} \Omega^{\prime}, j \Omega}^{J}\right|^{2}\right]^{1 / 2}} .
$$

(the number $d_{\Omega^{\prime} \Omega}^{J}(\theta)$ represents a reduced rotation matrix element [37]). The formulae above justify the choice of notation for the intrinsic reactants PDDCSs $\left(S_{q}^{(k)}(\theta)\right)$. These are obtained by nothing more than a transformation of the scattering matrix and, therefore, they can be interpreted as visualizable versions of the S matrix that make the stereodynamical information explicit.

\subsection{Reaction observables dependence on the $\mathrm{j}$ polarisation}

The previous Section introduced the intrinsic moments and, in particular, it pointed out their two key properties:

- the intrinsic moments represent the $\mathrm{BC}$ polarisation when the reaction takes place and

- quantify the reaction probability for any extrinsic polarisation of the reactants.

While the former characteristic allows for studying the collisions mechanism, the second makes possible to analyse the feasibility of controlling the reaction output through the reactants polarisation. 
This Section will be focused on developing the theoretical tools necessary to judge the magnitude and the limits of that control. These tools will consist of expressions for the reaction cross sections as a function of the extrinsic BC polarisation.

\subsubsection{Observable state-to-state differential cross section}

When dealing with reaction observables and, in consequence, with possible experimental situations, it will be necessary to consider a second frame of reference: the laboratory (or space fixed) frame $(X Y Z)$, whose origin coincides with the $x y z$ centerof-mass frame. Upper case letters will be used to represent vectors and moments defined with respect to the $X Y Z$ frame: $Q$ will label the polarisation moment components and upper case $\Theta_{j}$ and $\Phi_{j}$ will represent the polar and azimuthal angles that describe the direction of the rotational angular momentum vector $\mathbf{j}$.

Let us suppose that (as it is done in this work) one wants to study the reaction dynamics in the scattering frame $x y z$, and that all intrinsic properties are referred to $x y z$. If reactants are produced in the laboratory with complex polarization moments $A_{Q}^{(k)}$ referred to the space fixed frame $X Y Z$, then a frame transformation is required: one must use the values of the laboratory polarization moments $A_{Q}^{(k)}$ to determine the values of the center-of-mass frame polarization moments $a_{q}^{(k)}$. This transformation is given by (App. A)

$$
a_{q}^{(k)}=\sum_{Q=-k}^{k} D_{q Q}^{k *}(\alpha, \beta, \gamma) A_{Q}^{(k)}
$$

where $D^{k}(\alpha, \beta, \gamma)$ is a Wigner rotation matrix [37] and $\alpha, \beta$ and $\gamma$ are the Euler angles associated with the rotations that take the $x y z$ frame into the $X Y Z$ one ( $\beta$ and $\alpha$ are the polar and azimuthal angles that describe the orientation of $Z$ in the center-of-mass frame (see Fig. 4.2)).

The formula required for evaluating the state-to-state differential cross section (DCS) when the reactants are extrinsically polarised is (a derivation can be found in App. (C)

$$
\frac{d \sigma}{d \omega}=\frac{\sigma_{\text {iso }}}{2 \pi} \sum_{k q}(2 k+1)\left[S_{q}^{(k)}(\theta)\right]^{*} a_{q}^{(k)}
$$

where $d \sigma / d \omega$ is the DCS, $\sigma_{\text {iso }}$ is the integral cross section of the reaction involving unpolarized reactants (in other words, the reaction in which the experimental spatial distributions of the rotational angular momentum and internuclear axis of the BC molecule are isotropic), $S_{q}^{(k)}(\theta)$ are the state-to-state PDDCSs and $a_{q}^{(k)}$ are the 


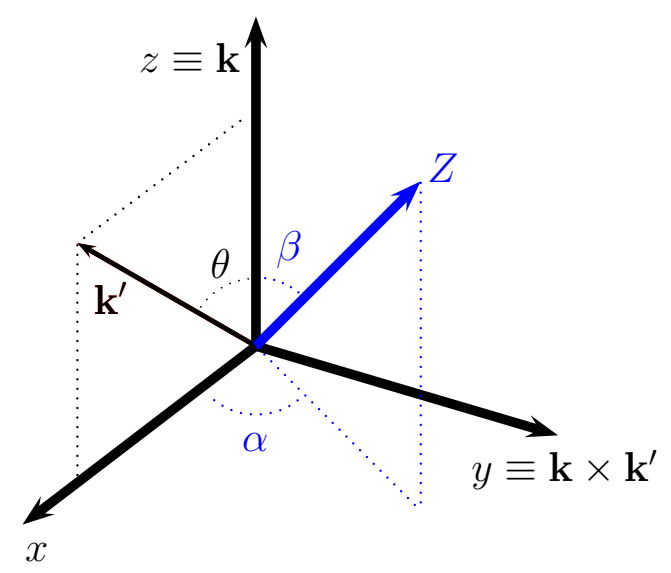

Fig. 4.2.: Relation between the laboratory frame $Z$ axis and the $x y z$ center-of-mass frame. Both frames are related through the $(\alpha, \beta, \gamma)$ Euler angles.

extrinsic moments ${ }^{3}$ (that is, the polarisation moments that describe the $\mathrm{BC}$ experimental polarisation in the center-of-mass frame of reference). This expression holds the same for classical or quantum mechanics with just a minimal difference: the upper limit of the sum is $\infty$ in the classical case and $2 j$ in the quantum mechanical case.

In general, the DCS given by Eq. (4.20) is not axially symmetric with respect to the $z(\mathbf{k})$ axis because it depends on the $\alpha, \beta$ and $\gamma$ angles (through the $a_{q}^{(k)}$ moments), that is, on the relative position of the laboratory frame with respect to the center-ofmass frame.

Eq. (4.20) makes possible to gain additional insight into the PDDCSs meaning:

- It sets explicitly how they quantify the probability of forming the $A B$ molecule with scattering angle $\theta$ when the $B C$ molecule extrinsic polarisation is given by the $k, q$ moment.

- In most of the experiments the reactants are unpolarised (they are not extrinsically prepared prior to reaction). This implies that the extrinsic polarisation moments must fulfill the condition (see subsection 2.3.3)

$$
a_{q}^{(k)}=\delta_{k 0} \delta_{q 0}
$$

and, in consequence, that the only nonzero moment is the $a_{0}^{(0)}$ one (its value represents the population of the state describing the experimental preparation

${ }^{3}$ In Chapter 2 the $a_{q}^{(k)}$ symbol was employed to represent a generic polarisation moment. From now on, it will represent the extrinsic moments 
of the reactants. In general, it will be assumed to be equal to one). The substitution of these moments into Eq. (4.20) provides with the following expression for the $S_{0}^{(0)}(\theta)$ PDDCS

$$
S_{0}^{(0)}(\theta)=\frac{2 \pi}{\sigma_{\text {iso }}} \frac{d \sigma_{\text {iso }}}{d \omega}(\theta)
$$

where $\frac{d \sigma_{i s o}}{d \omega}$ is the isotropic DCS, that only depends on the scattering angle (it is axially symmetric (see below)). This expression coincides [1] with that for the angular distribution (the reaction probability as a function of the scattering angle) when the reactants are unpolarised. By integrating over the scattering angle, it is trivial to confirm that (as anticipated) the $s_{0}^{(0)}$ polarisation parameter will always be equal to one.

\subsubsection{Observable state-to-state integral cross section}

The evaluation of the integral cross section (ICS) in the center-of-mass frame involves the sum of the DCS along all the directions in the space, that is, integration over the scattering angle $\theta$ and the azimuthal angle $\phi$ (Chapter 3). When the reactants are initially unpolarised or their extrinsic polarisation is such that the initial preparation is axially symmetric with respect to the $\mathbf{k}$ direction, the DCS is independent on $\phi$ and the corresponding integration is equivalent to multiply by $2 \pi$. Whenever this is not truth it is firstly necessary to answer the question "who is exactly $\phi$ ?" and then to perform the integration. Fortunately the response for the question is simple: $\phi$ is the angle between the scattering and the $z Z$ plane, that is, $\alpha$ (see Fig. 4.2). In consequence, the integral cross section for an arbitrary reactants polarisation can be directly determined from the expression 4.20 by integrating over $\theta$ and $\alpha$, obtaining the formula

$$
\sigma=\sigma_{\text {iso }} \sum_{k}(2 k+1) s_{0}^{(k)} a_{0}^{(k)}
$$

where the complex conjugate symbol has been dropped because the PPs with $q=0$ $\left(s_{0}^{(k)}\right)$ are real, the ICS dependence on the angles $\beta$ and $\gamma$ is not explicitly indicated and the upper limit of the sum is again $2 j(\infty)$ in the quantum mechanical (classical) case. According to this expression, the polarisation moments with $q \neq 0$ do not contribute to the ICS. This can be mathematically justified by having a look at the explicit dependence on the Euler angles of the rotation matrix involved in the LAB to center-of-mass moments transformation (Eq. (4.19) ) 37.

$$
D_{q Q}^{k *}(\alpha, \beta, \gamma)=e^{i q \alpha} d_{q Q}^{k}(\beta) e^{i Q \gamma}
$$


It is evident that integration of the first complex exponential over the $\alpha$ range of values ( $[0,2 \pi])$ cancels unless $q=0$. Physically, the no contribution of the $q \neq 0$ moments to the ICS can be rationalised by thinking about their relation to the cartesian axis (Sec. 2.4 and references therein): the $q=0$ moments do not depend on the location of the scattering plane (for instance, the $k=2, q=0$ moment determines the angular momentum alignment with respect to the $z$ axis) while those corresponding to $q \neq 0$ assume known of the location of that plane. The determination of the ICS involves integration over the scattering and azimuthal angles or, in other words, it includes to sum over all the possible positions of the scattering plane. Therefore, after performing such integration it becomes completely meaningless to talk about the scattering plane and the ICS cannot depend on moments which are referred to it.

Of course, the range of observable quantities is not completely made up by the DCS and the ICS. In particular, it is possible to conceive an "special" cross section $(\tilde{\sigma})$ obtained by integration only over the scattering angle and whose value would be given by

$$
\tilde{\sigma}=\sigma_{\text {iso }} \sum_{k q}(2 k+1)\left[s_{q}^{(k)}\right]^{*} a_{q}^{(k)}
$$

In contrast with $\sigma$, the special ICS depends on all the polarisation moments. The reason for this difference lies in that $\tilde{\sigma}$ determination does not involve integration over $\alpha$ but, on the contrary, it requires specification of the location of the scattering plane. Therefore, its values will depend on the angles $\beta$ and $\gamma$ (as $\sigma$ ) but also on the azimuthal angle $\alpha$. Experimentally, the special ICS can only be indirectly measured, by integration of a (directly measurable) differential cross section. It cannot be determined when product detection does not discriminate between different recoil directions $\mathbf{k}^{\prime}$.

\subsubsection{Total observables}

The state-to-state control expressions formerly introduced can be generalised to deal with observables corresponding to a well defined set of final states. In particular, the combination of Eq. (4.20), (4.23) and (4.25) with the definitions of the total PDDCSs $S_{q, \text { tot }}^{(k)}(\theta)$ (Eq. (4.12)) and the total PPs $s_{q \text {,tot }}^{(k)}($ Eq. (4.13) $)$ provides with the following formulas for the total DCS, ICS and special ICS respectively

$$
\frac{d \sigma_{\mathrm{tot}}}{d \omega}=\frac{\sigma_{\mathrm{iso}, \mathrm{tot}}}{2 \pi} \sum_{k q}(2 k+1)\left[S_{q, \mathrm{tot}}^{(k)}(\theta)\right]^{*} a_{q}^{(k)}
$$




$$
\begin{gathered}
\sigma_{\text {tot }}=\sigma_{\text {iso,tot }} \sum_{k}(2 k+1) s_{0, \text { tot }}^{(k)} a_{0}^{(k)} \\
\tilde{\sigma}_{\text {tot }}=\sigma_{\text {iso,tot }} \sum_{k q}(2 k+1)\left[s_{q, \text { tot }}^{(k)}\right]^{*} a_{q}^{(k)},
\end{gathered}
$$

where

$$
\sigma_{\text {iso }, \text { tot }}=\sum_{v^{\prime}, j^{\prime}} \sigma_{\text {iso }}\left(v^{\prime}, j^{\prime}\right)
$$

(for the sake of clarity, the dependence of $\sigma_{\text {iso }}$ on the final state has been explicitly indicated). The analysis of the total observables dependence on the extrinsic $\mathbf{j}$ polarisation gives the most general overview of the reaction control possibilities and it is a suitable starting point for this kind of studies.

\subsubsection{Enhancement numbers}

Equations introduced so far allow for quantifying the extent to which reactivity is affected by reactants extrinsic polarisation. The definition of the reactivity enhancement number (or suppression number if the extrinsic polarisation diminishes the reactivity)

$$
n=\ln \left(A_{\mathrm{pol}} / A_{\text {iso }}\right) .
$$

(where $A$ represents any of the considered reaction observables (DCS, ICS or special ICS) when the reactants are initially polarised $\left(A_{\text {pol }}\right)$ or unpolarised $\left.A_{\text {iso }}\right)$ facilitates the analysis of the results as it leads to a single scale for the reactivity change.

The reason for using $\ln$ in the enhancement number definition is to ensure that positive (negative) values of $\mathrm{n}$ will correspond to increases (decreases) of the reaction observable magnitude and that changes of the same relative importance will always take to $n$ values with the same absolute value. This can be illustrated through an example based on supposing two different experimental polarisations such that one of them leads to a 5 -fold increment in the reaction DCS and the other to 5 -fold decrease. In the fist case the $n$ number will be $\ln (5)$ and in the second $-\ln (5)$, that is, the same absolute value but different sign. On the contrary, if ln were not used, the $n$ values related to both polarisations would be 5 and 0.2 .

\section{Theoretical quantum mechanical limits for the enhancement number}

When the $\mathrm{A}+\mathrm{BC}$ reaction is studied from the quantum mechanical point of view it is possible to determine the theoretical limits of the $n$ values before performing any calculation. 
The largest and the smallest accessible values of the enhancement number must correspond to pure states of the reactants, that is, to extrinsic polarisations of the reactants which correspond ${ }^{4}$ to states that are fully described by a single ket:

$$
\left|\phi_{\max }\right\rangle=\sum_{\Omega=-j}^{j} c_{\Omega}|j \Omega\rangle
$$

for the pure state taken to the largest value of the observable and

$$
\left|\phi_{\min }\right\rangle=\sum_{\Omega=-j}^{j} c_{\Omega}^{\prime}|j \Omega\rangle
$$

for the pure state taken to the smallest value of the observable. For the purpose of proving this statement, let us analyse the situation if the largest/smallest cross section value were associated to the reactants mixed state represented by the density operator

$$
\hat{\rho}=\frac{1}{N}\left(\left|\phi_{1}\right\rangle\left\langle\phi_{1}|+| \phi_{2}\right\rangle\left\langle\phi_{2}|+\ldots+| \phi_{N}\right\rangle\left\langle\phi_{N}\right|\right)
$$

instead of corresponding to a pure state. In this case, two possibilities open up:

- The reactants extrinsic preparation corresponding to each pure state $\left(\left|\phi_{r}\right\rangle\right)$ which takes part in the mixture does not lead to the same cross section. This situation can never occur because it is not compatible with the assumption that the extreme values of the reaction observable correspond to mixed reactants states. Labelling the pure state of the mixture which takes to the largest (smallest) cross section as $\left|\phi_{\max }\right\rangle\left(\left|\phi_{\min }\right\rangle\right)$, it is evident that a preparation of the reactants according exclusively to $\left|\phi_{\max }\right\rangle\left(\left|\phi_{\min }\right\rangle\right)$ will take to a larger (smaller) reaction output than the preparation according to the mixture.

- Although extremely improbable, it could be found that the cross section corresponding to the extrinsic polarisation related to each of the pure states taking part in the mixture $\left(\left|\phi_{r}\right\rangle\right)$ is the same. If this were the case, the preparation of the reactants according to one of those pure states instead of according to the mixture also takes to the extreme value of the reaction observable. In consequence, in order to quantify the extreme observable value it is enough to determine one of such pure states and to evaluate the corresponding cross section.

As the first possibility can not happen because it leads to a contradiction and the

\footnotetext{
${ }^{4}$ Eq. (2.43) provides with the connection between the state and the polarisation moments
} 
second is equivalent to consider a pure state, it is concluded that the limit values of the cross section only can be reached by extrinsically preparing the reactants in a pure state (which will necessarily evolve into a pure products state (see discussion in Sec. (2.2)).

Therefore, in order to evaluate the largest possible enhancement and suppression of the cross section by reactants polarisation, the generation of a pure, completely coherent products state is considered. This situation is realised, for instance, by the

$$
\mathrm{A}+\mathrm{BC}(v, j) \rightarrow \mathrm{AB}\left(v^{\prime}, j^{\prime}=0\right)+\mathrm{C}
$$

reaction when product detection specifies a particular scattering angle. The values of the $v, j$ and $v^{\prime}$ quantum numbers, although left unspecified, are assumed to be fixed; the only controllable parameters are those specifying the polarisation of the reactants rotational angular momentum, $\mathbf{j}$. The $\mathrm{AB}+\mathrm{C}$ state in reaction (4.34) is pure and it can be represented by the ket $\left|j^{\prime}=0 \Omega^{\prime}=0\right\rangle$. As a pure state only can evolve to or being evolved from a pure state, the $\mathrm{A}+\mathrm{BC}$ reactants should be in a pure state as well. This pure state is a linear combination of the $|j \Omega\rangle$ kets and it will be represented by $|1\rangle$. While $|1\rangle$ is an eigenstate of $\hat{\jmath}^{2}$, this is not necessarily true for $\hat{\jmath}_{z}$. The other elements of an orthonormal basis formed by $\hat{j}^{2}$ eigenstates and containing $|1\rangle$ will be denoted as $|p\rangle, p=2,3,4, \ldots, 2 j+1$.

The importance of the $|p\rangle, p=1,2,3, \ldots, 2 j+1$ basis lies in that:

- The reactants polarisation that maximizes the DCS of reaction (4.34) at a particular $\theta$ is the one corresponding to $|1\rangle$ (the reaction must proceed necessarily through this pure state and the closer to it is the reactants preparation the higher will be the reactivity). This maximal DCS value will be indicated as $d \sigma_{1} / d \omega$.

- The minimal DCS is the one associated with reactants in any linear combination of the $\hat{\jmath}^{2}$ eigenstates orthogonal to $|1\rangle$, and its value is zero. This is because, by definition, the overlap between $|1\rangle$ and each of the $|p\rangle, p=2,3,4, \ldots, 2 j+1$ states is zero.

The "isotropic" DCS is obtained with unpolarised reactants. In other terms, with a completely incoherent superposition of the $2 j+1$ polarisation states $(|p\rangle, p=$ $1,2,3, \ldots, 2 j+1)$. As the DCS associated with the first of those is $d \sigma_{1} / d \omega$ and the 
others are all zero, the isotropic DCS is given by

$$
\frac{d \sigma_{\text {iso }}}{d \omega}=\frac{1}{2 j+1} \sum_{p=1}^{2 j+1} \frac{d \sigma_{p}}{d \omega}=\frac{1}{2 j+1} \frac{d \sigma_{1}}{d \omega}
$$

which establishes the minimum and maximum values of the reactivity enhancement number for pure state reactions (those connecting a defined pure state of the reactants with a pure state of the products):

$$
\begin{array}{lll}
\frac{d \sigma_{\max } / d \omega}{d \sigma_{\text {iso }} / d \omega}=2 j+1 & \Rightarrow & n_{\max }=\ln (2 j+1), \\
\frac{d \sigma_{\min } / d \omega}{d \sigma_{\text {iso }} / d \omega}=0 & \Rightarrow & n_{\min }=\ln 0=-\infty
\end{array}
$$

It will be proved as follows that these limits hold indeed for $\mathrm{A}+\mathrm{BC}$ pure reactions where $j^{\prime}$ is different from zero and also for reactions where the reactants and products are in mixed states. The differences with the $j^{\prime}=0$ case will not lie in the value of the theoretical limits but in the almost null probability of approaching them (while for $j^{\prime}=0$ "all" what has to be done to reach the largest or smaller value of $\mathrm{n}$ is to determine the $\mathbf{j}$ polarisation corresponding to the $|p\rangle$ states and to prepare the reactants according to them, in the rest of the cases it will be necessary to determine the suitable reactants polarisation and also to wait for the fulfilling of several additional conditions that make it extremely improbable).

If $j^{\prime} \neq 0$ in reaction (4.34), the isotropic DCS can be expressed as a sum of $2 \min \left(j, j^{\prime}\right)+1$ DCSs (Eq. (4.35) is an special case of this decomposition)

$$
\frac{d \sigma_{\text {iso }}}{d \omega}=\frac{1}{2 j+1} \sum_{p=1}^{2 \min \left(j, j^{\prime}\right)+1} \frac{d \sigma_{p}}{d \omega}
$$

where each one of them corresponds to a pure state reaction and, in contrast with the $j^{\prime}=0$ case, it is in general different from 0 . The largest one represents the maximum accessible DCS for the reaction and, at most, it can be equal to $2 j+1$ times the isotropic DCS. However, for this value to be reached it is not only necessary to prepare the reactants in the pure state taking to that maximum DCS but to find that all the other pure state reaction DCSs in Eq. (4.37) cancels simultaneously. As this is quite improbable, this limit will be very rarely approached. Regarding the minimum value of the DCS, it is necessary to differentiate between two possible situations

- $j \leq j^{\prime} \rightsquigarrow$ The minimum reaction DCS is that corresponding to the smallest 
term in the sum (4.37). In general it will be different from zero.

- $j>j^{\prime} \rightsquigarrow$ The minimum reaction DCS is necessarily zero. The $2 j^{\prime}+1$ reactants pure states corresponding to the $2 j^{\prime}+1$ pure state reactions participating in decomposition (4.37) form a subspace of the reactants states space. Any state contained in the orthogonal complement to that subspace represents a possible reactants state (and therefore a reactants polarisation) which does not leads to products formation.

It is necessary as well to consider products mixed states originated by incoherent superpositions. The possible sources of incoherence are:

- Integration over the scattering angle (this leads to incoherent superposition of the different partial waves associated with different values of the total angular momentum).

- Neglect of other coherences between product rotational or vibrational states (this arises when products are detected regardless of their rotational or vibrational state and the results therefore correspond to a simple sum over such states).

All of these are likely (as a matter of fact, practically guaranteed) to reduce the extent to which reactant polarisation can enhance or suppress (except when $j>j^{\prime}$, see above) reactivity. The reason is that the reactant polarisation that maximises or minimises reactivity into a given pure state is unlikely to have the same effect on all of the other pure states taking part in the incoherent superposition.

It therefore holds that, in general,

$$
-\infty \leq n \leq \ln (2 j+1)
$$

and that is it practically impossible to obtain the equalities in measurements involving mixed states.

To end with, it is convenient to analyse what is the relation between the intrinsic quantum mechanical moments (PPs and PDDCSs) and the upper limits of the enhancement numbers. As was pointed out in Sec. 4.3, the intrinsic moments represent the reactants polarisation when the reaction takes place. However, this does not mean that by extrinsically preparing the reactants according to the intrinsic polarisation the reaction output will be automatically maximised. In fact, two different cases can be found: 
- The reaction must be necessarily a pure state reaction (what only happens when the products are detected at a defined scattering angle and $j^{\prime}=0$ (Eq. (4.34)) or when, by pure chance, only one of the pure states that can participate in the process turns out to be reactive). In this case the intrinsic polarisation (given by the corresponding intrinsic moments) must coincide with the polarisation of the pure state which gives rise to reaction $(|1\rangle$ in the notation previously used) and it will maximise the reaction observable.

- The reaction can take place through more than one pure state. In this case, the intrinsic moments (state-to-state PDDCSs for $j^{\prime} \neq 0$, total PDDCSs or PPs) do not coincide with the reactants polarisation corresponding to the pure state leading to the highest value of the reaction observable but they represent an average of the reactants polarisations related to each one of the pure state reactions participating in the process. In consequence, the intrinsic polarisation will not maximise the reaction observable. The reason for the non correspondence between the intrinsic moments and the "best" pure state polarisation comes from the fact that, intrinsically, the reaction can not have a bias towards any of the reactive pure states. However, it is possible to extrinsically force the reaction to happens through a certain pure state just by suitably preparing the reactants. Eventually, this could take to a larger value of the reaction observable than the preparation according to the intrinsic polarisation.

Regardless of whether it takes to a maximum value or not, the extrinsic preparation of the reactants according to the intrinsic polarisation will always take to values of the observables larger than the isotropic ones, that for the state-to-state reactions are:

$$
\begin{gathered}
\frac{d \sigma}{d \omega}=\frac{\sigma_{\text {iso }}}{2 \pi} \sum_{k q}(2 k+1)\left|S_{q}^{(k)}(\theta)\right|^{2} \\
\sigma=\sigma_{\text {iso }} \sum_{k}(2 k+1)\left|s_{0}^{(k)}\right|^{2}
\end{gathered}
$$

and

$$
\tilde{\sigma}=\sigma_{\text {iso }} \sum_{k q}(2 k+1)\left|s_{q}^{(k)}\right|^{2}
$$

Quantum mechanical determination of the largest and smallest accessible values of the enhancement number

Last pages discussion has proved than the values of the enhancement quantum number are comprised into the $[-\infty, \ln (2 j+1)]$ interval. In practise, the extremes of 
the interval are reached in only a few special cases. Whenever this does not happens, it is necessary to have a procedure to establish how close to the limits it is possible to get by controlling the extrinsic reactants polarisation. To expose such a procedure is the goal of this Section.

The calculation will be facilitated by the fact that, regardless of wether they reach the interval limits or not, the maximum and the minimum values of the cross section must always correspond to pure states of the reactants. In other words, the reactants extrinsic preparation when the extreme values of the cross section are reached will be that corresponding to a single $|\psi\rangle$ ket or, equivalently, to the density operator

$$
\hat{\rho}=|\psi\rangle\langle\psi|
$$

By using the $|\psi\rangle$ expansion in terms of the $|j \Omega\rangle$ basis

$$
|\psi\rangle=\sum_{\Omega_{1}=-j}^{j} c_{\Omega_{1}}\left|j \Omega_{1}\right\rangle \quad\left(\langle\psi|=\sum_{\Omega_{2}=-j}^{j} c_{\Omega_{2}}^{*}\left\langle j \Omega_{2}\right|\right)
$$

(remember that $\Omega$ represents the reactants helicity) the density operator can be rewritten as

$$
\hat{\rho}=\sum_{\Omega_{1}=-j}^{j} \sum_{\Omega_{2}=-j}^{j} c_{\Omega_{1}} c_{\Omega_{2}}^{*}\left|j \Omega_{1}\right\rangle\left\langle j \Omega_{2}\right|
$$

The extrinsic reactants preparation given by Eq. (4.44) corresponds to the polarisation moments

$$
a_{q}^{(k)}=\sum_{\Omega_{1}, \Omega_{2}=-j}^{j} c_{\Omega_{1}} c_{\Omega_{2}}^{*}\left\langle j \Omega_{1} k q \mid j \Omega_{2}\right\rangle
$$

(see Eq. (2.43)). The substitution of these moments in Eq. (4.20), Eq. (4.23) and Eq. (4.25) (or in their "total" counterparts) provides with expressions for the reactions observables in terms of the $c_{\Omega}$ expansion coefficients (more specifically, in terms of their real $\left(R_{\Omega}\right)$ and imaginary $\left(I_{\Omega}\right)$ parts). For instance, the state-to-state integral cross section can be written as

$$
\sigma=\sigma_{i s o} \sum_{\Omega_{1}=-j}^{j}\left[\sum_{k=0}^{2 j}\left((2 k+1) s_{0}^{\{k\}}<j \Omega_{1} k 0 \mid j \Omega_{1}>\right)\right]\left(R_{\Omega_{1}}^{2}+I_{\Omega_{1}}^{2}\right)
$$

where the $s_{0}^{\{k\}}$ coefficients represent the real state-to-state polarisation parameters.

Thanks to equations like that, the problem of evaluating the limit values of the cross section has been transformed into an extreme calculation problem in $2(2 j+1)$ 
dimensions (the space of the $R_{\Omega}$ and $I_{\Omega}$ coefficients) which can be tackled by means of the "Multidimensional Downhill Simplex Method" algorithm 69. The algorithm is useful for the quest of minima and works by moving the vertices of a "simplex" (a geometrical figure consisting, in $N$ dimensions, of $N+1$ vertices) over the hypersurface corresponding to the function whose minimum is being searched until that extreme is enclosed into the simplex. The calculation ends when the differences between the values of the cross section in the vertices is smaller than a certain parameter whose value can be externally controlled. If the target function is multiplied by minus one, its maxima transform in minima and vice versa. This way, the same algorithm can be applied as well to the search of maxima.

The method application needs to be slightly modified because the variables are not independent but related through the equation

$$
\sum_{\Omega=-j}^{j}\left(R_{\Omega}^{2}+I_{\Omega}^{2}\right)=1
$$

that represents the $|\psi\rangle$ normalisation (and it corresponds to a reactants population equal to one). The fulfilling of this condition can be ensured by normalizing the variables according to it prior to each call of the function (the function whose extremes are being calculated) by the algorithm subroutine.

\subsection{Stereodynamical portraits}

The former Section has been focused on reaction control through the polarisation of the reactants and on how to quantify that control by using the intrinsic moments (PPs and PDDCSs). However, the utility of these moments goes beyond the control analysis as they represent the reactants polarisation when the reaction takes place (Section 4.3 ) and, therefore, they express the directional preferences of the reaction. This means that the study of the information contained in the intrinsic moments (in particular in the real renormalised PDDCSs and the real PPs) can provide with a description of the reaction mechanism. The problem is how to extract such a description. One could consider separately each polarisation moment (symmetry and intensity of the polarisation). However, this procedure is not convenient as it requires a considerable mental gymnastic (the distortions of the unpolarised (spherical) distribution associated with the various polarisation moments are not trivial) to visualise the information contained in the moments. Instead of considering separately the meaning of every single moment it would be more convenient to obtain a global 
picture that combine all their contributions. This can be achieved by means of the stereodynamical portraits, which condense the information contained in all the moments (making unnecessary to analyse individually every polarisation moment) and provide with "pictures" of the mechanism; that is, with visual representations of the reaction dynamics dependence on the directions in the space.

The present Section will be devoted to the presentation of the stereodynamical portraits. Although they will be introduced employing quantum mechanical (Sec. 4.5.1) and classical (Sec. 4.5.2) intrinsic polarisation moments (because, in this work, the portraits will be generally used in conjunction with them), it is necessary to point out that, given any set of polarisation moments (not necessarily intrinsic), it is possible to use the portraits to visualise the directional effects of the angular momentum polarisation. For instance, if extrinsic moments were employed, the corresponding portraits would represent the $\mathrm{BC}$ molecule experimental preparation.

\subsubsection{Quantum mechanical portraits}

Assuming that the whole set of quantum mechanical intrinsic polarisation moments is known, two different kinds of portraits can be defined. One of them (the molecular axis portraits) represents the molecular axis distribution leading to products formation in well resolved states, partially or totally summed over final states and/or integrated over scattering angles depending on the particular set of moments employed. Complementary, it also can be defined portraits referring to the distribution of the $\mathrm{BC}$ molecule rotational angular momentum (rotational portraits) giving rise to reaction.

The molecular axis portraits related to a set of PPs are given by the probability density function (see App. $\mathrm{D}$ for a derivation)

$$
P_{r}\left(\theta_{r}, \phi_{r}\right)=\sum_{k=0}^{2 j} \sum_{q=-k}^{k} \frac{2 k+1}{4 \pi} s_{q}^{(k)}\langle j 0, k 0 \mid j 0\rangle C_{k q}^{*}\left(\theta_{r}, \phi_{r}\right)
$$

where $\theta_{r}$ and $\phi_{r}$ are the polar and azimuthal angles that describe the position of $\mathbf{r}$ in the centre-of-mass reference frame, $\langle j 0, k 0 \mid j 0\rangle$ is a Clebsch-Gordan coefficient, $C_{k q}^{*}\left(\theta_{r}, \phi_{r}\right)$ are the complex conjugates modified spherical harmonics and $s_{q}^{(k)}$ represent the set of PPs (state-to-state or total). The portrait gives us the reaction probability when $\mathbf{r}$ lyes along the direction given by $\theta_{r}, \phi_{r}$ or, in other words, the internuclear axis distribution when the reaction (whose intrinsic moments are the $s_{q}^{(k)}$ set) takes place. 
Similarly, the molecular axis portraits corresponding to reaction into a given scattering angle can be defined

$$
P_{r}\left(\theta_{r}, \phi_{r} \mid \cos \theta\right)=\sum_{k=0}^{2 j} \sum_{q=-k}^{k} \frac{2 k+1}{4 \pi} \frac{S_{q}^{(k)}(\theta)}{S_{0}^{(0)}(\theta)}\langle j 0, k 0 \mid j 0\rangle C_{k q}^{*}\left(\theta_{r}, \phi_{r}\right)
$$

where in this case the renormalised PDDCSs have been used. Eq. (4.49) represents the conditional reaction probability at a given scattering angle $\theta$ when the internuclear axis points along $\theta_{r}, \phi_{r}$. If, instead, the joint reaction probability as a function of $\theta_{r}$, $\phi_{r}$ and $\theta$ would be considered, the $S_{q}^{(k)}(\theta)$ PDDCSs had to be used

$$
P_{r}\left(\theta_{r}, \phi_{r}, \cos \theta\right)=\sum_{k=0}^{2 j} \sum_{q=-k}^{k} \frac{2 k+1}{4 \pi} S_{q}^{(k)}(\theta)\langle j 0, k 0 \mid j 0\rangle C_{k q}^{*}\left(\theta_{r}, \phi_{r}\right)
$$

To clarify the difference between using or not renormalised PDDCSs to generate the portraits it is convenient to remember the discussion about the necessity of renormalising the moments before comparing the polarisation of physical systems with different population (Sec. 2.4.3). Through the renormalisation the information regarding the population is eliminated from the moments and all of them are referred to the same scale (the scale corresponding to systems with population equal to one), allowing for their direct comparison. In the present case, the population is given by the $S_{0}^{(0)}(\theta)$ moment and the orthogonality of the spherical harmonics allows for proving that the volume of the portraits 5 coincides with the $k=0$ moment or, in other words, that it is equal to 1 when renormalised PDDCSs are used (Eq. 4.49) and equal to $S_{0}^{(0)}(\theta)$ otherwise (Eq. 4.50). So, to renormalise the PDDCSs is equivalent to transform the portraits in figures with volume equal to one. This transformation does not distort the shape of the figures but refers their features to the same volume, in such a way that the portraits for different values of $\theta$ can be meaningfully compared. This is the reason why, in the future, renormalised PDDCSs will always be employed to generate the portraits corresponding to defined values of the scattering angle.

In an analogous way, the rotational angular momentum portraits are given by the quantum PDFs defined in Sec. 2.5

$$
Q_{r}\left(\theta_{j}, \phi_{j}\right)=\sum_{k=0}^{2 j} \sum_{q=-k}^{k} \frac{2 k+1}{4 \pi} s_{q}^{(k)}\langle j j, k 0 \mid j j\rangle C_{k q}^{*}\left(\theta_{j}, \phi_{j}\right)
$$

\footnotetext{
${ }^{5}$ The volume of the portraits is defined as their integral over all the values of $\theta_{r}$ and $\phi_{r}$. Remember that when a function $f(x, y)$ is integrated over a $(x, y)$ region, the result corresponds to the volume contained under the function in that region
} 
As it was pointed out in Chapter $2, Q\left(\theta_{j}, \phi_{j}\right)$ is not a probability density function but a population distribution. Strictly speaking, the values of the rotational portraits are not the reaction probabilities when the vector $\mathbf{j}$ is pointing along the direction specified by $\theta_{j}$ and $\phi_{j}$ (defined in the centre-of-mass frame) but they give the population of the minimum uncertainty state (the state in which the projection of $\mathbf{j}$ takes the maximum value, that is, $j$ ) lying along the direction given by $\theta_{j}$ and $\phi_{j}$ when the reaction happens. In the correspondence limit of high $j$ values the $Q_{r}$ can be considered as probability density functions. As in the case of the molecular axis portraits, if the rotational portraits are meant to represent the polarisation taken to reaction at a given scattering angle, the renormalised PDDCSs must be used instead of the PPs.

Whereas the molecular axis portraits give a more intuitive description of the collision geometry (they describe it in terms of the relative position of the atoms), rotational portraits give a description that is more complete. This is so because orientation moments (polarisation moments with odd $k$ ) do not contribute to molecular axis distributions. Mathematically this ensues from the fact that whereas $\langle j 0, k 0 \mid j 0\rangle$ (the Clebsch-Gordan coefficient in the molecular axis portraits) vanishes whenever $k$ is odd, $\langle j j, k 0 \mid j j\rangle$ (the Clebsch-Gordan coefficient in the rotational portraits) does not. The physical explanation is that angular momentum orientation does not induce molecular axis orientation. Thinking in classical terms, the $\mathbf{r}$ rotation around $\mathbf{j}$ averages out any orientation of the axis even when the angular momentum is oriented.

It is necessary to point out that the former paragraph discussion does not mean that the internuclear axis can not be oriented. In fact, it can be oriented by employing pendular states ${ }^{6}$ generated by means of external electric fields [99, 100, 101]. However, the preparation of pendular states requires coherence between different $j$ states and, in (rovibrational) state-to-state reactions (whose stereodynamical study is the goal of this work), that is not possible. (Rovibrational) state-to-state reactions only allow for coherence between helicity eigenstates. This can lead to angular momentum orientation and alignment, and also to molecular axis alignment, but not to molecular axis orientation.

\subsubsection{Quasiclassical portraits}

When one does not have a set of quantum mechanical moments but a classical one it is also possible to generate the corresponding stereodynamical portraits. Following the discussion presented in Sec. 2.5, the right procedure to visualise the symmetries contained in the classical moments consist of substituting them in the quantum

\footnotetext{
${ }^{6}$ Directional hybrids of the field-free rotational states, in which the molecular axis librates about the electric field vector
} 
mechanical expressions for the portraits, instead of employing purely classic infinite series like Eq. (2.2) (and its counterpart for the internuclear axis). This provides with quasiclassical portraits, where the classical results have been turned into quasiclassical by forcing onto them a quantum mechanical restriction whose effect is to restrict the extent to which the exact direction of the angular momentum can be specified [39, 40]. This way, the non-dynamical information related to the consideration or not of the uncertainty principle is removed (the quasiclassical portraits can be compared with their quantum mechanical counterparts and the differences will be exclusively due to dynamical differences and not to the consideration or not of the uncertainty principle).

\subsection{Experimental feasibility of the proposed measurements}

This section describes a currently feasible crossed-beam experiment that could be used to test the theoretical predictions obtained through the tools introduced in the previous Sections. The experiment presentation will be particularized for the

$$
\mathrm{H}+\mathrm{D}_{2}(v=0, j=2) \rightarrow \mathrm{D}+\mathrm{HD}
$$

reaction. There are several reasons for selecting this particular example:

- Calculations for the $\mathrm{H}+\mathrm{D}_{2}$ system are feasible and reliable.

- The parameters necessary for planning the experiment (susceptibility to stimulated Raman excitation, depolarisation constants, etc) are known.

- In Sec. 4.7 it will be shown that theory predicts $\mathrm{D}_{2}$ alignment to have a dramatic effect on the collision outcome, and that this effect will be clearly visible in differential cross sections, integral cross sections and product state distributions. As the proposed experiment can lead to the measurement of all of these quantities, it will be capable of unambiguously demonstrating those theoretical predictions.

However, it has to be pointed out that the $\mathrm{H}_{3}$ system is by no means the only system that can be examined with the proposed experiment. On the contrary, it allows for the study of many reactions. The only essential requirement will be that the molecule to be polarised must be amenable to stimulated Raman excitation. Its collision partner 

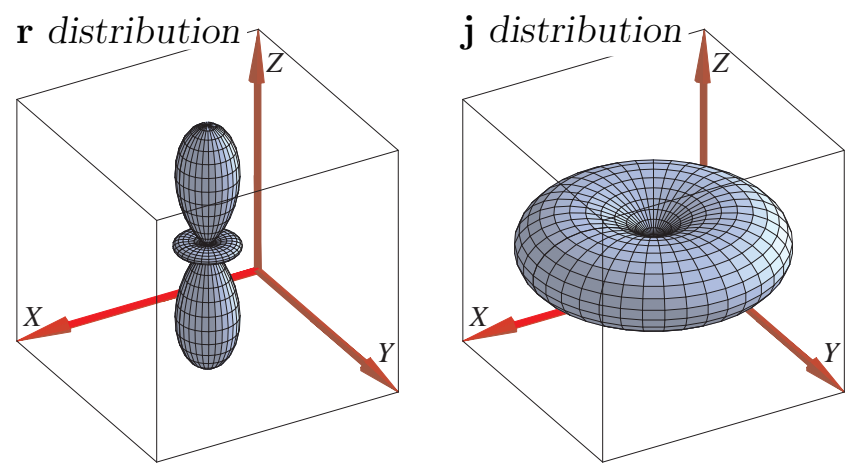

Fig. 4.3.: Spatial (extrinsic) distributions of the interatomic axis, $\mathbf{r}$, and the rotational angular momentum, $\mathbf{j}$, of a diatomic molecule in a $|j=2, m=0\rangle$ state referred to the laboratory frame, $X Y Z$. The centre of the figure coincides with the origin of the frame. However, the last has been displaced in order to facilitate the figure visualisation.

does not even need to be an atom, although if comparisons to theory are to be made, atom-diatom reactions are the systems of choice.

As it will be made clear below, the experiment utility goes beyond checking the validity of the theoretical predictions. Its conceptual simplicity and the intuitive interpretation of the extrinsic preparations in terms of different kinds of collisions allow as well for its employment as a modelling tool.

In simple terms, the experiment consists in the following: to place the molecules in the $\mathrm{D}_{2}$ beam in the $|v=0, j=2, m=0\rangle$ state, where the magnetic quantum number is determined with regard to a laboratory-fixed quantization axis $Z$ whose direction can be chosen. This extrinsic preparation provides with $\mathrm{D}_{2}(v=0, j=2)$ molecules whose interatomic axis is aligned parallel/antiparallel to $Z$ (positive axial alignment) and whose rotational angular momentum is aligned perpendicular to $Z$ (negative rotational alignment), see Fig. 4.3 (these figures are the quantum mechanical portraits corresponding to the $|v=0, j=2, m=0\rangle$ state and referred to the laboratory frame). By varying the direction of the laboratory axis $Z$, one varies its direction with regard to the scattering-frame vector $\mathbf{k}$ and, if the experiment involves angle-resolved product detection, also with regard to $\mathbf{k}^{\prime}$ and therefore with regard to the scattering plane (the plane containing $\mathbf{k}$ and $\mathbf{k}^{\prime}$ ). Given that the laboratory axis $Z$ is also the axis with regard to which the $\mathrm{D}_{2}$ molecules are aligned, changing the direction of $Z$ with regard to $\mathbf{k}$ and possibly $\mathbf{k}^{\prime}$ amounts to changing the $\mathrm{D}_{2}$ alignment in the centre-ofmass frame ( $x y z$, see Fig. 4.2), and this is the basic idea of the experiment. Some of the technicalities are discussed further below, but first it will briefly reconsidered 
the problem of how to express the laboratory $\mathbf{r}$ and $\mathbf{j}$ distributions in the scattering frame.

The axial and rotational $\mathrm{D}_{2}$ polarizations are both completely described by the extrinsic molecular polarization moments. Following the notation used in Sec. 4.4.1, these moments will be represented by $A_{Q}^{(k)}$ when referred to the $X Y Z$ laboratory frame. In the case considered here the only nonvanishing moments are those with $k=0,2$ or 4 and $Q=0$ (the distributions are axially symmetric with respect to the $Z$ axis), and they take the values ${ }^{7}$

$$
A_{0}^{(0)}=1, \quad A_{0}^{(2)}=-\sqrt{\frac{2}{7}}, \quad A_{0}^{(4)}=+\sqrt{\frac{2}{7}}
$$

(As appropriate for an experiment, the values above are quantum mechanical. The corresponding classical values are $A_{0}^{(0)}=1, A_{0}^{(2)}=-1 / 2$ and $A_{0}^{(4)}=3 / 8$.)

In order to obtain the extrinsic reactants polarization moments in the $x y z$ scattering frame, all one has to do is to use Eq. (4.19). It leads to

$$
a_{q}^{(k)}=D_{q 0}^{k *}(\alpha, \beta, \gamma) A_{0}^{(k)}=C_{k q}(\beta, \alpha) A_{0}^{(k)} .
$$

Where, as usual, $\alpha, \beta$ and $\gamma$ are the Euler angles corresponding to the rotation which transform the $x y z$ frame into the $X Y Z$ one. Note that, as the considered distributions have cylindrical symmetry around $Z$ and therefore all nonvanishing $A_{Q}^{(k)}$ moments have $Q=0$, the Euler angle $\gamma$ does not play any role in the transformation and can be arbitrarily chosen. The only Euler angles required are $\alpha$ and $\beta$, the azimuthal and polar angles that specify the direction of the laboratory axis $Z$ in the scattering frame (Figs. 4.1 and 4.2 present a graphical illustration of how these angles are defined).

In order to make easier the visualisation of the axis preparations that can be achieved by means of this procedure, some examples for significant values of $\beta$ and $\alpha$ are included in Fig. 4.4. The distributions are worked out by combining the portraits expressions (Eqs. 4.48 and 4.51) with the $a_{q}^{(k)}$ moments introduced in Eq. 4.54 (except for the couple of figures labeled as "isotropic" (unpolarised reactants), which correspond to $\left.a_{q}^{(k)}=\delta_{k 0} \delta_{q 0}\right)$ and, therefore, all the distributions will be referred to the $x y z$ scattering frame. It is useful to remember that the $x y z$ frame is such that the reactants approach direction is along $z$ and that the products recoil direction lies on the $x z, x \geq 0$ half-plane (that is, $x z$ is the scattering plane). This involves that

\footnotetext{
${ }^{7}$ Obtained by substituting the density operator which describes the extrinsic preparation $\hat{\rho}=|j=2, m=0\rangle\langle j=2, m=0|$
}

into Eq. (2.43) 

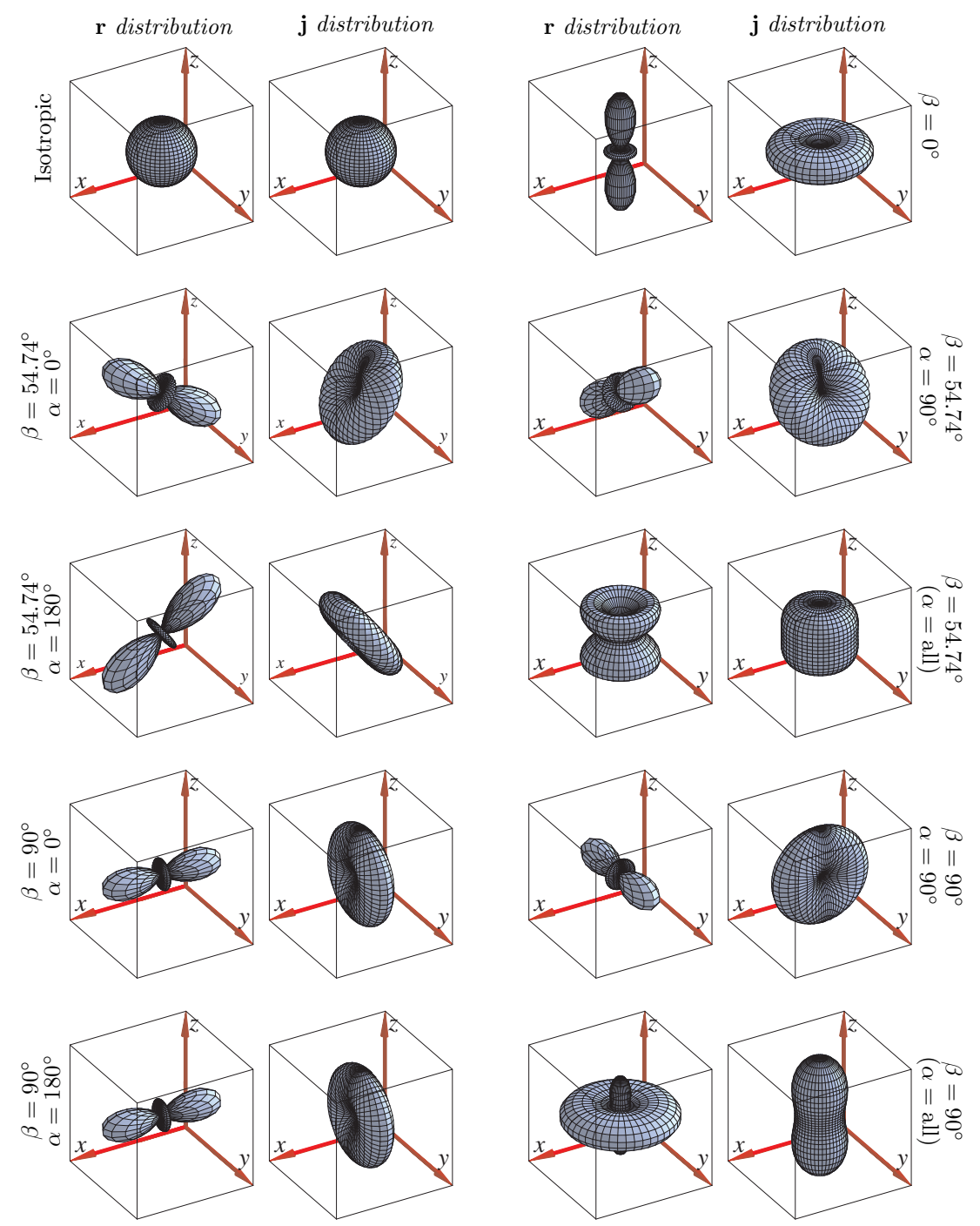

Fig. 4.4.: Spatial (extrinsic) distributions of the $\mathbf{r}$ and $\mathbf{j}$ axis corresponding to unpolarised (isotropic) and aligned (including the $\beta$ and $\alpha$ values used to generate molecular alignment) reactants. All the figures are referred to the centre-ofmass frame (xyz) and the axis have been displaced from the center of each figure as in Fig. 4.3 
- $\beta=0^{\circ}$ amounts to head-on collisions (it is equivalent to prepare the reactants in the $|j=2, \Omega=0\rangle$ state).

- $\beta=90^{\circ}$ amounts to side-on collisions.

- $\beta=54.74^{\circ}$ (this is the value of the "magic angle", that is, the angle which makes zero the second Legendre polynomial) represents an intermediate situation between the two previous cases.

and that

- $\alpha=0$ and $180^{\circ}$ amounts to collisions where the internuclear is initially on the scattering plane.

- $\alpha=90^{\circ}$ corresponds to collisions where the axis was prepared perpendicular to the scattering plane.

The distributions for $\beta=0^{\circ}, \beta=54.74^{\circ}(\alpha=$ all $)$ and $\beta=90^{\circ}(\alpha=$ all $)$ display axial symmetry with respect to the $z$ axis (only the $a_{q=0}^{(k)}$ moments contribute to their shape). They are examples of extrinsic preparations when the integral cross section is measured and integration over all the spatial directions is performed. The symmetry stems from the fact that, as a consequence of the integration over the azimuthal angle values, the scattering plane is completely undefined (Sec.4.4.2) and, therefore, the axis preparations can not depend on $\alpha$. Mathematically, the moments that characterise the distributions in this case are obtained by averaging the $a_{q}^{(k)}$ set over the $\alpha$ values

- $\beta \neq 0^{\circ} \rightarrow$ the moments averaged over $\alpha$ are obtained through the integral

$$
\frac{1}{2 \pi} \int_{0}^{2 \pi} a_{q}^{(k)} d \alpha=\frac{C_{k q}(\beta, 0) A_{0}^{(k)}}{2 \pi} \underbrace{\int_{0}^{2 \pi} e^{i q \alpha} d \alpha}_{2 \pi \delta_{q 0}}
$$

and it is found that only the $a_{q=0}^{(k)}$ moments "survive" to the average process.

- $\beta=0^{\circ} \rightarrow$ the average would not be necessary in this case because $C_{k q}(0, \alpha) \propto$ $\delta_{q 0}$ and only the $a_{q=0}^{(k)}$ moments can be different from zero.

The preparation of the $|j=2, m=0\rangle$ state can be achieved by pure rotational Raman scattering by selecting the right pump and Stokes laser frequencies for stimulated Raman scattering in a cell of $\mathrm{D}_{2}$. By excitation via the $\mathrm{S}(0)$ transition from $\mathrm{D}_{2}(v=0, j=0)$, a considerable population of $\mathrm{D}_{2}(v=0, j=2, m=0)$ can be produced quite effectively by setting the polarizations of the stimulated Raman pump 
and Stokes lasers parallel to each other. The procedure proposed here is very similar to that used by Kandel et al. 97] in their study of the $\mathrm{Cl}+\mathrm{HD}(v=1, j=2)$ reaction. Sitz and coworkers have used a similar procedure to produce aligned $\mathrm{N}_{2}$ in the $v=1$ state 102 .

Associated with the $j=2$ rotational state of $\mathrm{D}_{2}$ are nuclear states with total spin $T=0$ and $T=2$, which can in principle lead to very strong hyperfine depolarization [29, 31, 38]. This effect, however, is not expected to be significant under the experimental conditions considered here. The reason is that collisions will occur within nanoseconds of reactants preparation but hyperfine depolarization will only occur in a microseconds time scale. Justification of this claim requires consideration of the largest separation between $\mathrm{D}_{2}(j=2)$ hyperfine energy levels $(185 \mathrm{kHz}$, see ref [103) and of the fact that the time scale for hyperfine depolarization [31, 104, 105] can not be shorter than the reciprocal of that value, that is, no shorter than $5 \mu \mathrm{s}$. This result is confirmed by detailed calculations [106, 107.

In order to minimize the presence of unpolarized $\mathrm{D}_{2}(v=0, j=2)$ in the beam, one can expand pure $o-\mathrm{D}_{2}$ through a nozzle cooled to liquid nitrogen temperature. This is known to produce more than $95 \%$ of $\mathrm{D}_{2}$ molecules in the $v=0, j=0$ state. Although, at best, only $50 \%$ of the $\mathrm{D}_{2}$ molecules can be excited into $|j=2, m=0\rangle$, the data acquisition can be done on a shot-to-shot basis with the excitation laser on/off or varying the polarizations of the pump and Stokes lasers to be either perpendicular or parallel.

Ideally, the experiment will be carried out in a high resolution crossed molecular beam apparatus, similar to those used by Welge and coworkers [108] and by Yang and coworkers [109], with a well defined scattering plane. By varying the direction of the polarization vector $\mathbf{E}$ (which plays the role of the $Z$ axis) with respect to the relative velocity vector in $\beta$ and the scattering plane in $\alpha$, different reactant polarizations in the $x y z$ frame can be achieved. Results from time-of-flight detection of the products at different laboratory scattering angles can be transformed into the center-of-mass system to obtain state resolved differential cross sections and angle-recoil velocity polar maps. Moreover, by integrating the triple DCS (polar maps) in scattering angle and velocity, the special integral cross section could be determined for each geometry with different $\beta$ and $\alpha$ angles.

This section is concluded by noting that the formulae necessary for the theoretical calculation of the state-to-state cross sections that can be measured in this experiment are

$$
\frac{d \sigma_{\alpha}^{\beta}}{d \omega}=\frac{\sigma_{\text {iso }}}{2 \pi} \sum_{k q}(2 k+1)\left[S_{q}^{(k)}(\theta)\right]^{*} C_{k q}(\beta, \alpha) A_{0}^{(k)},
$$




$$
\begin{gathered}
\sigma^{\beta}=\sigma_{\text {iso }} \sum_{k}(2 k+1) s_{0}^{(k)} C_{k 0}(\beta, 0) A_{0}^{(k)} . \\
\tilde{\sigma}_{\alpha}^{\beta}=\sigma_{\text {iso }} \sum_{k q}(2 k+1)\left[s_{q}^{(k)}\right]^{*} C_{k q}(\beta, \alpha) A_{0}^{(k)},
\end{gathered}
$$

These formulae are obtained by insertion of Eq. (4.54) in Eqs. (4.20), (4.23) and (4.25), respectively. Note that, contrary to the special ICS $\tilde{\sigma}_{\alpha}^{\beta}$, the ICS $\sigma^{\beta}$ is independent of $\alpha$. The mathematical reason for this is that the value of $C_{k 0}(\beta, \alpha)$ is independent of $\alpha$; the physical reason was outlined in connection with the Fig. 4.4 distributions and thoroughly discussed in Sec. 4.4.2. Note also that, as this experiment involves $\mathrm{D}_{2}$ alignment but not orientation, one can restrict the $\beta$ and $\alpha$ ranges to

$$
\begin{aligned}
& 0^{\circ} \leq \beta \leq 180^{\circ}, \\
& 0^{\circ} \leq \alpha \leq 180^{\circ},
\end{aligned}
$$

and that the $\beta$ range can be further reduced to

$$
0^{\circ} \leq \beta \leq 90^{\circ}
$$

when the $\alpha$-dependence is averaged out.

\subsection{Illustrative examples}

There are three ways in which one can use the theory described in the previous Sections. For reasons explained below, they will be called as "intrinsic," "practical" and "min-max" approaches. Each one of them has its own particular advantages, and the stereodynamical analyses will be easiest when all three are combined.

The $\mathrm{H}+\mathrm{D}_{2}$ reaction will be now used to illustrate the potential of each of those approaches. As "the simplest of chemical reactions", the $\mathrm{H}_{3}$ system has been regarded as the benchmark system in chemical reactions dynamics and as the first choice for tests of new approaches to the problem. This has led to an impressive number of accurate theoretical and experimental results allowing for thorough descriptions of many aspects of its dynamics (described in a recent review [110]). However, very few of these works [25, 98, have considered the reaction stereodynamics. The examples presented in this Section, together with the contents of next Chapter, are expected to shed light on that reaction facet.

In particular, both quantum and quasiclassical numerical results have been obtained 
for the

$$
\mathrm{H}+\mathrm{D}_{2}(v=0, j=2) \rightarrow \mathrm{HD}\left(v^{\prime}, j^{\prime}\right)+\mathrm{D}
$$

process at collision energies up to $E_{\text {coll }}=1.7 \mathrm{eV}$. The dynamical calculations - timeindependent quantum reactive scattering and quasiclassical trajectories (the general characteristics of both methodologies can be found in Chapter 3) - were ran on the BKMP2 potential energy surface [111]. The scattering matrices employed on the quantum mechanical analysis were worked out by Dr. Brian Kendrick, from "Los Alamos National Laboratory" (New Mexico, USA) [112.

Besides choosing a particular reaction, the examples have been selected so that they highlight what can be achieved with the possible experiment described in the former Section.

It is necessary to stress, however, that the main purpose of this presentation is not to allow for a detailed analysis of the experiment or even of the role of $\mathrm{D}_{2}$ polarization in reaction (4.61). Instead, the main purpose here is to provide illustrative examples of the kind of chemical information one can obtain by using the reactants polarization theory that has been described.

\subsubsection{The intrinsic approach: insight and understanding}

When one wants to focus on the reaction stereodynamics itself rather than on a particular reactive process, the most natural way to tackle the problem is to use the intrinsic approach described here. This approach ignores completely the extrinsic factor (the actual polarization of reactants) and concentrates exclusively on the detailed inspection of the intrinsic reactants polarisation. In the terms of the metaphor used in Sec. 4.2, the intrinsic approach analyses what the reaction wants (intrinsic polarisation) rather than what it gets (extrinsic polarisation). By means of this analysis of the reaction preferences one can answer the following questions

- what is the sensitivity of the reaction to reactants polarization?

- what polarizations give rise to reaction?

- how anisotropic is the reaction dynamics?

- what is the correlation between reactants polarization and reactivity?.

The key idea which sustains this approach has already been discussed: intrinsic moments (PDDCSs and PPs) represent the reactants polarisation when the reaction happens or, in other words, they are the polarization moments of the reaction itself, 
rather than the polarization moments of actually existing reactants. If we know these intrinsic polarization moments, we can combine them with the "stereodynamical portraits" to plot the corresponding internuclear axis and rotational angular momentum distributions (this leads to clear pictures of the reaction mechanism itself, some of them of a rather intuitive nature) or to analyse them individually (to perform a more quantitative study).

Figs. 4.5 and 4.6 show some of these portraits, obtained with quantum polarisation moments. In each of them, the left column shows molecular axis portraits, while the right column shows rotational portraits ${ }^{8}$. Fig. 4.5 shows results integrated over the scattering angle for the

$$
\mathrm{H}+\mathrm{D}_{2}(v=0, j=2) \rightarrow \mathrm{HD}\left(v^{\prime}=0, j^{\prime}\right)+\mathrm{D}
$$

reaction with $j^{\prime}=5$ or $j^{\prime}=10$ and at $E_{\text {coll }}=1.306 \mathrm{eV}$, while Fig. 4.6 shows $\theta$ dependent results for the

$$
\mathrm{H}+\mathrm{D}_{2}(v=0, j=2) \stackrel{\theta}{\rightarrow} \mathrm{HD}\left(v^{\prime}=0, j^{\prime}=0\right)+\mathrm{D}
$$

reaction obtained with $\theta=4^{\circ}, 8^{\circ}$ or $11^{\circ}$ and at the same collision energy. In other words, Fig. 4.5 shows the $P\left(\theta_{r}, \phi_{r}\right)$ and $Q\left(\theta_{j}, \phi_{j}\right)$ functions obtained through use of the intrinsic PPs [the $s_{q}^{(k)}$ s] , while Fig. 4.6 shows the same functions obtained through use of the intrinsic renormalized PDDCSs [the $S_{q}^{(k)}(\theta) / S_{0}^{(0)}(\theta)$ 's].

Pictures such as those in Figs. 4.5 and 4.6 give the best possible representations of what back in 1990 Levine [113] called the "chemical shape" (as opposed to "physical shape") of a chemical reaction, and as such fulfill one of the goals of chemical reaction stereodynamics: to determine the "shapes" of atoms and molecules as perceived by each other when they take part in a reactive collision.

Figs. 4.5 and 4.6 also justify the use of the words "insight" and "understanding" in the heading of this section. A brief consideration of each of them does give significant insight of the reaction stereochemistry, and also facilitates understanding of its mechanism. For instance, Fig. 4.5 leads very naturally to the hypothesis that the amount of rotational energy of the HD product in reaction (4.62) is related to the collision geometry, with head-on reactive collisions leading to little product rotational excitation ( $c f$. the $j^{\prime}=5$, top row of Fig. 4.5) and side-on reactive collisions leading to larger product rotational excitation ( $c f$. the $j^{\prime}=10$, bottom row of Fig. 4.5). A detailed discussion of this reaction feature will be included in next Chapter (devoted

${ }^{8}$ The portraits, as the corresponding intrinsic moments, are referred to the center of mass frame 


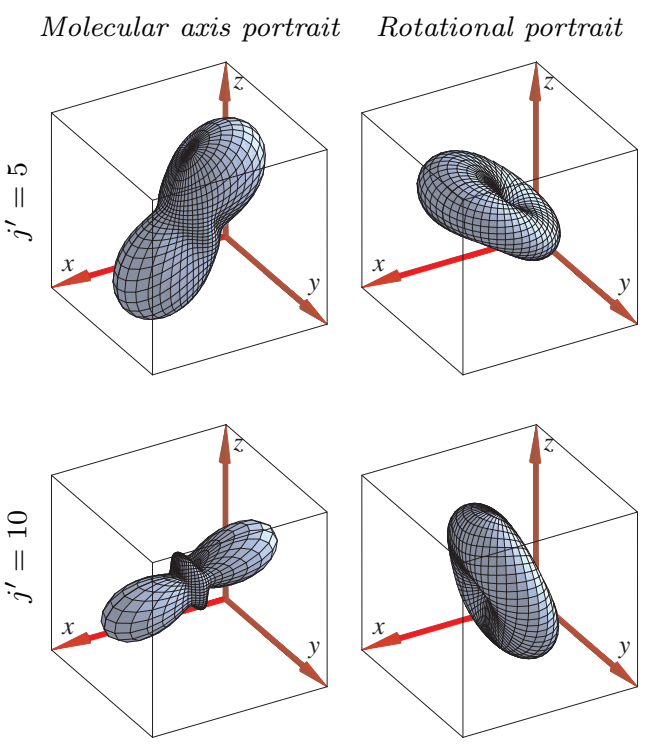

Fig. 4.5.: Stereodynamical portraits revealing the "chemical shape" of reaction (4.62) at $E_{\text {coll }}=1.306 \mathrm{eV}$ with $j^{\prime}=5$ (top) or $j^{\prime}=10$ (bottom). The portraits on the left (right) column show the distributions of the $\mathrm{D}_{2}$ interatomic axis (rotational angular momentum) when reaction (4.62) happens. For the sake of clarity, the axes have been displaced from the center of each of the figures.

to the analysis of the $\mathrm{H}+\mathrm{D}_{2}$ mechanism through this approach). Anticipating some results, it will be found that this effect has to do with the amount of bending energy of the collision complex in the transition state region.

Ideal as they are in terms of qualitative understanding, the stereodynamical portraits presented above are not suitable for quantitative analyses. In quantitative terms, the key ingredients of the intrinsic approach to reaction stereodynamics are the intrinsic polarization-dependent differential cross sections (PDDCSs) and polarization parameters (PPs) introduced in Sec. 4.3, which are the numerical parameters behind the stereodynamical portraits presented above.

Figs. 4.7 and 4.8 show PPs and renormalized PDDCSs calculated again at the $E_{\text {coll }}=1.306 \mathrm{eV}$ collision energy. Each of these pictures includes both quantum and quasiclassical data, which allows for a quantitative assessment of the level of agreement between the two calculations.

Fig. 4.7 show real PPs of rank $k=1$ and 2 (these are the real moments whose directional information is easier to visualise (see Sec. 2.4) and, in consequence, the most suitable for direct analysis) obtained for reaction (4.62) as a function of the 

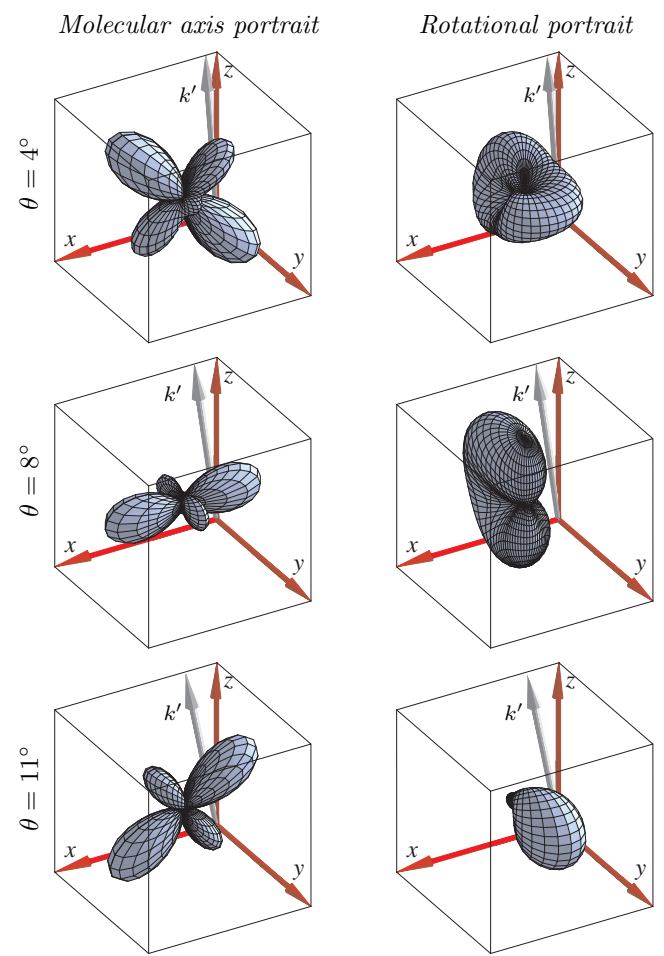

Fig. 4.6.: Stereodynamical portraits revealing the "chemical shape" of reaction (4.63) at $E_{\text {coll }}=1.306 \mathrm{eV}$, and with $\theta=4^{\circ}$ (top), $\theta=8^{\circ}$ (middle), or $\theta=11^{\circ}$ (bottom). The portraits on the left (right) column show the distributions of the $\mathrm{D}_{2}$ interatomic axis (rotational angular momentum) when reaction (4.63) happens. The scattering angle $\theta$ between the reactant-approach and product-recoil directions is the angle between the vectors $\mathbf{k} \equiv z$ and $\mathbf{k}^{\prime}$. For the sake of clarity, the axes have been displaced from the center of each of the figures.

HD product rotational state. As can be seen, the agreement between the quantum and quasiclassical PPs is impressive. Furthermore, these figures show that the polarization parameter $s_{0}^{\{2\}}$ is the most relevant one for the differences found between the stereodynamical portraits of reactions leading to $\operatorname{HD}\left(j^{\prime}=5\right)$ or $\operatorname{HD}\left(j^{\prime}=10\right)$ (see Fig. 4.5). The $s_{0}^{\{2\}}$ parameter is the one whose value changes the most between $j^{\prime}=5$ and $j^{\prime}=10$, going from rather negative at $j^{\prime}=5$ (its quantum value is -0.18 , while the negative limit for $j=2$ is -0.53 (see App. B) ) to rather positive at $j^{\prime}=10$ (its quantum value is 0.16 , while the positive limit for $j=2$ is +0.53 (see App. B)). As moments with $k=2$ and $q=0$ are indicative of alignment of the rotational angular momentum vector with respect to the quantization axis $z$, the observation that 

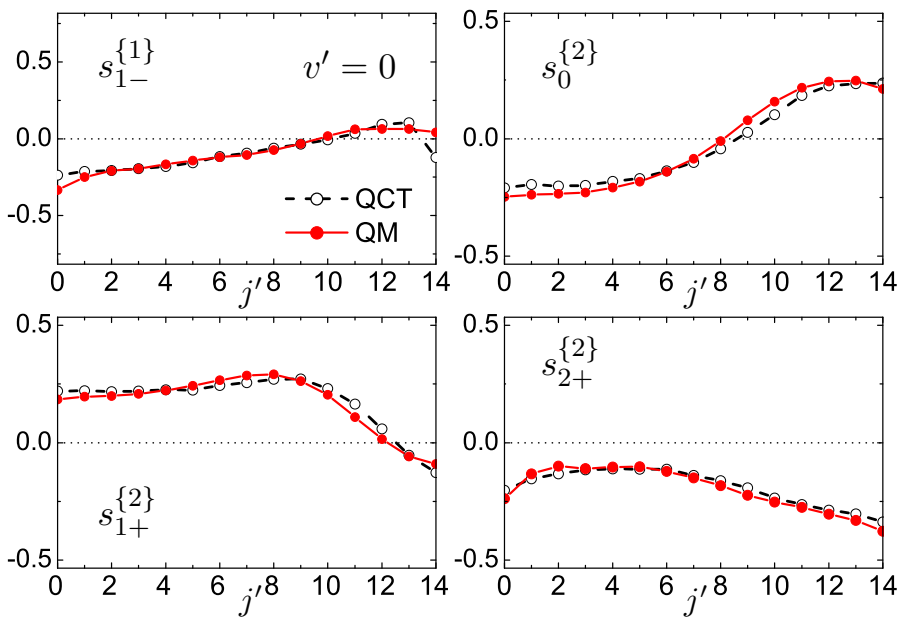

Fig. 4.7.: QM (solid line, solid circles) and QCT (dashed line, open circles) intrinsic polarization parameters of reaction (4.62) with $k=1,2$ at $E_{\text {coll }}=1.306 \mathrm{eV}$ as a function of the HD product rotational state. The ranges of the vertical axes coincide with the allowed quantum ranges of these parameters for $j=2$.

the $s_{0}^{\{2\}}$ parameter is so important for the reaction dynamics strongly suggests that experiments involving reactant rotational alignment are likely to shed considerable light on the reaction dynamics. The possible experiment we described in Sec. 4.6 is precisely of this kind, and we will further explore the impact of reactant rotational alignment on measurable quantities.

Fig. 4.8 shows intrinsic renormalized PDDCSs of rank $k=2$ and component $q=0$ as a function of the scattering angle for selected $\operatorname{HD}\left(v^{\prime}=0, j^{\prime}\right)$ product states. Before commenting on these results one must remember that these renormalized PDDCCs are not indicative of reaction probability, but rather of the preferred reactant polarization at each scattering angle. As the reaction probability itself does change with scattering angle, one must take the product angular distributions into account when analyzing the renormalized PDDCSs.

Formation of $\operatorname{HD}\left(v^{\prime}=0, j^{\prime}=1\right)$ is dominated by backward scattering, but there is also significant forward scattering. Consideration of Fig. 4.8 shows that the agreement between quantum and quasiclassical data, while quite good in the backward scattering region, is not so good in the forward scattering region, where the QCT calculations fail to reproduce the pronounced oscillations of the quantum PDDCS. This information is interesting, and suggests a purely quantum origin for the observed oscillation, which is also seen in the stereodynamical portraits of Fig. 4.6 (the shape 

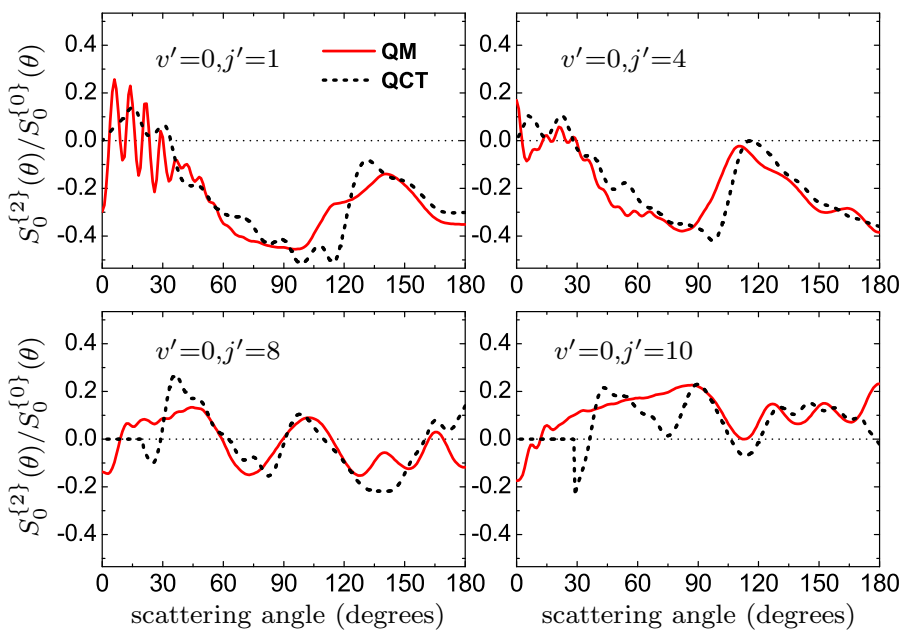

Fig. 4.8.: Intrinsic renormalized PDDCSs of rank $k=2$ and component $q=0$ of reaction (4.63) into $v^{\prime}=0, j^{\prime}=1,4,8$ or 10 at $E_{\text {coll }}=1.306 \mathrm{eV}$ as a function of scattering angle.

of the portraits changes very broadly for values of the angle which differ in just a few degrees). An exhaustive analysis of the oscillations will be postponed until next Chapter, where they will be attributed to an interference effect, as that found by Althorpe and coworkers [96, 114] in their plane wavepacket analysis of this reaction; the interference might be between near-side and far-side reaction mechanisms [115, 116].

In the case of the other product states included in Fig. 4.8, the dynamics is dominated by backward and/or sideways scattering. In these regions the agreement between quantum and quasiclassical data is generally good, although not as quantitative as the one found for the polarization parameters. As one might expect, the more detailed reaction properties (the PDDCSs) constitute a more stringent test of the accuracy of the calculations than the less detailed ones (the PPs).

\subsubsection{The practical approach: experimental control possibilities}

Sec. 4.7.1 focused on the analysis of intrinsic stereodynamical properties, obtained without consideration of actual reactants polarizations. While that method can give understanding and insight into the reaction stereodynamics, it cannot predict the outcome of practical situations and actual experiments. In order to deal with these, one needs the practical approach which is now described.

The "practical approach" is largely a trial-and-error procedure, in which reaction 
outcomes are determined with different reactants polarizations, and the corresponding results compared. The idea is to examine the extent to which the reaction outcome (say, its cross section or the product state distribution) can be influenced by actual reactants polarization schemes, and the extent to which one can use such polarization to (passively) control the reaction.

As described in Sec. 4.6 the proposed experiment involves $\mathrm{D}_{2}$ alignment with regard to the reactants approach direction $\mathbf{k}$ and possibly the products recoil direction as well. With this in mind, the examples have been restricted to alignment effects. It should be noted, however, that this is not a necessary restriction and orientation effects could be easily included in the theoretical examples.

In the experiment that has been proposed, the alignment direction is determined by $\beta$ and $\alpha$, the polar and azimuthal angles specifying the direction of the electric field vector in $x y z$ (the scattering frame of reference, see Secs. 4.3 and 4.6). Two different possibilities will be considered here

- cases in which both of these angles are specified (this is appropriate for experiments involving angularly-resolved product detection, and therefore for measurements of DCSs and special ICSs) and

- cases in which only $\beta$ is determined, while $\alpha$ is averaged out (this is appropriate for measurements of ordinary ICSs).

The values considered for the polar and the azimuthal angle are

$$
\beta=0^{\circ}, 54.74^{\circ} \text { (magic angle) } 0 \text { } 90^{\circ} \text {. }
$$

and

$$
\alpha=0^{\circ}, 45^{\circ}, 90^{\circ} \text { or } 180^{\circ} .
$$

When analyzing the data presented below, it will be useful to remember that the $D_{2}$ interatomic axis $\mathbf{r}$ and rotational angular momentum $\mathbf{j}$ are respectively aligned along or perpendicular to the direction specified by $\beta$ and $\alpha$ (see Fig. 4.4).

Fig. 4.9 shows quantum data illustrating the effect of the polar angle $\beta$ on the excitation function (the integral cross section $\sigma^{\beta}$, summed over all product states, as a function of the collision energy). The solid line corresponds to the usual excitation function (isotropic case, no $\mathrm{D}_{2}$ polarization), while the others include the effect of $\mathrm{D}_{2}$ alignment. The dependence of the reaction cross section on reactant alignment is clear: reactivity is enhanced by head-on, collinear collisions $\left(\beta=0^{\circ}, \mathbf{r}\right.$ and $\mathbf{j}$ respectively parallel and perpendicular to $\mathbf{k}$ ), diminished by side-on collisions ( $\beta=$ 


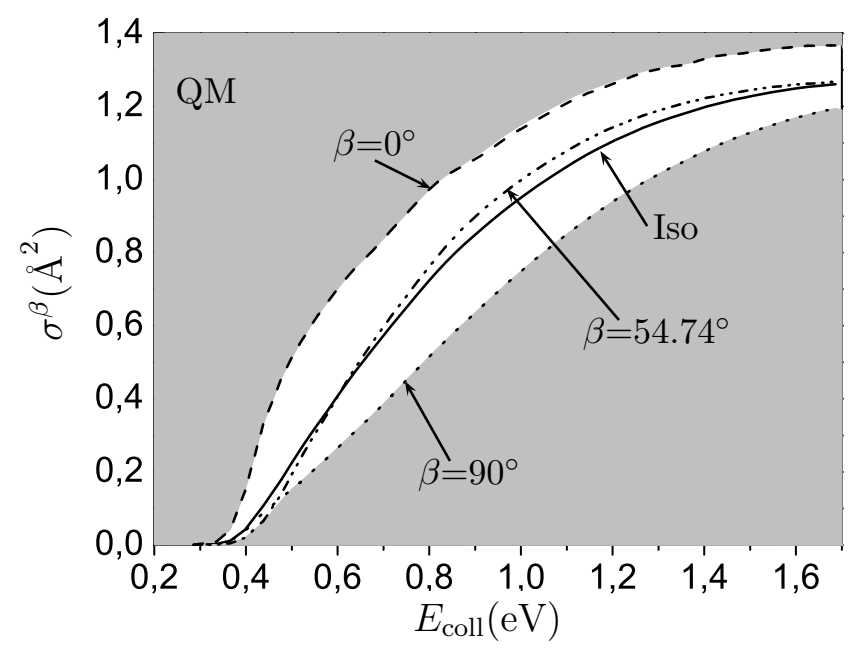

Fig. 4.9.: Excitation function of the $\mathrm{H}+\mathrm{D}_{2}(v=0, j=2)$ reaction for different $\mathrm{D}_{2}$ alignment directions. The curve labelled as "iso" corresponds to the situation in which $\mathrm{D}_{2}$ is unpolarized and the initial $\mathbf{j}$ vector is therefore random. The borders between the white and gray areas represent the maximum and minimum possible values of $\sigma^{\beta}$, and were obtained with the minmaximization procedure that will be described in Sec. 4.7.3.

$90^{\circ}, \mathbf{r}$ and $\mathbf{j}$ respectively perpendicular and parallel to $\mathbf{k}$ ), and largely unaffected by alignment along the magic angle. While this is not exactly an unexpected result for the reaction we are considering here (the $\mathrm{H}+\mathrm{D}_{2}$ reaction has long been known to be collinearly-constrained), one should note that Fig. 4.9, besides revealing a preference for collinear collisions, also quantifies it, showing the extent to which the reactivity can be controlled by selective reactant polarization.

The effect of $\mathrm{D}_{2}$ alignment on product rotational state distributions at $E_{\text {coll }}=$ $1.306 \mathrm{eV}$ (the HD vibrational state is $v^{\prime}=0$ ) is clearly seen in Fig. 4.10, which shows quasiclassical (top) and quantum (bottom) data, obtained with the same $\beta$ values considered above. The two data sets are in very good agreement, indicating a clear effect: collinear collisions $\left(\beta=0^{\circ}\right)$ lead to a colder product rotational state distribution, while side-on collisions $\left(\beta=90^{\circ}\right)$ make it hotter, and magic-angle alignment again leads to results similar to those obtained without reactant polarization. Consideration of these results, along with the stereodynamical portraits of Fig. 4.5, suggests that low/high $j^{\prime}$ values are associated with collisions with low/high impact parameters, and thus with transition states with lower/higher bending vibrational energy (more solids arguments on favour of this connection will be presented in next Chap- 

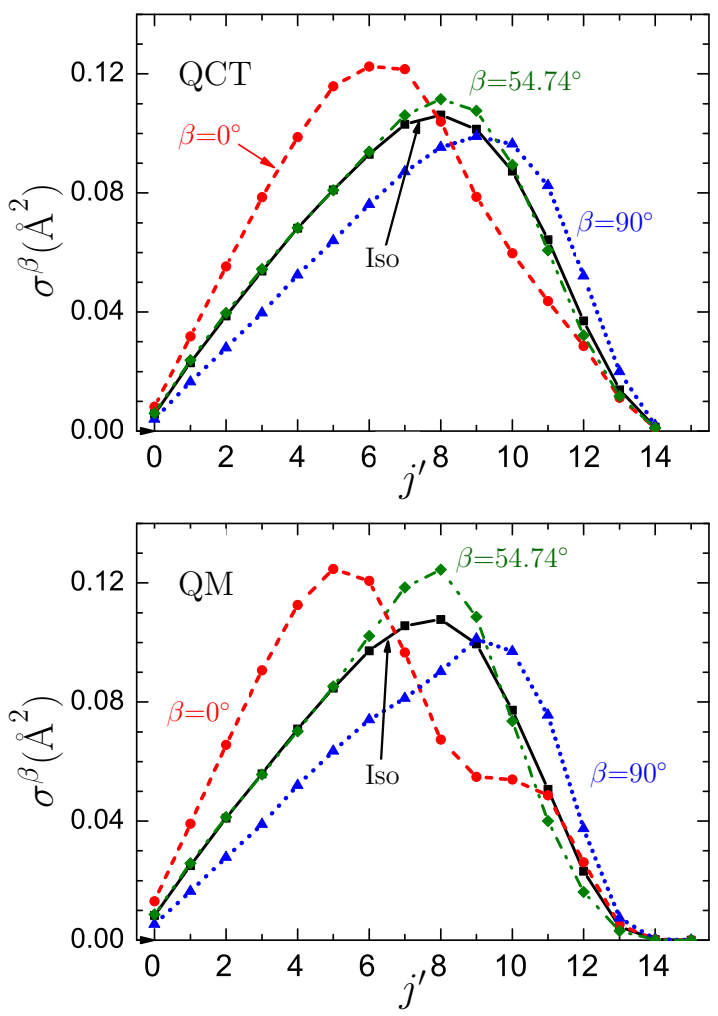

Fig. 4.10.: Integral cross section of reaction (4.62) at $E_{\text {coll }}=1.306 \mathrm{eV}$ as a function of the product rotational state for different $\mathrm{D}_{2}$ alignment directions. Top panel shows quasiclassical results, bottom panel quantum mechanical ones.

ter). Note that the qualitative behaviour of the cross sections corresponding to $j^{\prime}=5$ and 10 could be predicted without any calculation, just by comparing the intrinsic portraits of Fig. 4.5 with their extrinsic counterparts in Fig. 4.4. The reason is that the extrinsic preparations which bear more resemblance to the intrinsic portraits will be more reactive (because what the reaction "receives" is closer to what the reaction "wants") and vice versa. In this case, the experimental distributions for $\beta=0^{\circ}$ $\left(\beta=90^{\circ}\right)$ are more similar to the intrinsic portraits for $j^{\prime}=5\left(j^{\prime}=10\right)$ than to those for $j^{\prime}=10\left(j^{\prime}=5\right)$.

Comparison of the rotational distributions of Fig. 4.10 with the polarization parameters of Figs. 4.7 is also illustrative. The first thing to note is that, as the product recoil direction is not specified, we have cylindrical symmetry around the reactant-approach direction k: as shown by Eqs. (4.23) and (4.57), the only polarization parameters that contribute to the integral cross section are $s_{0}^{\{0\}}, s_{0}^{\{2\}}$ and $s_{0}^{\{4\}}$; of these, $s_{0}^{\{2\}}$ is the 


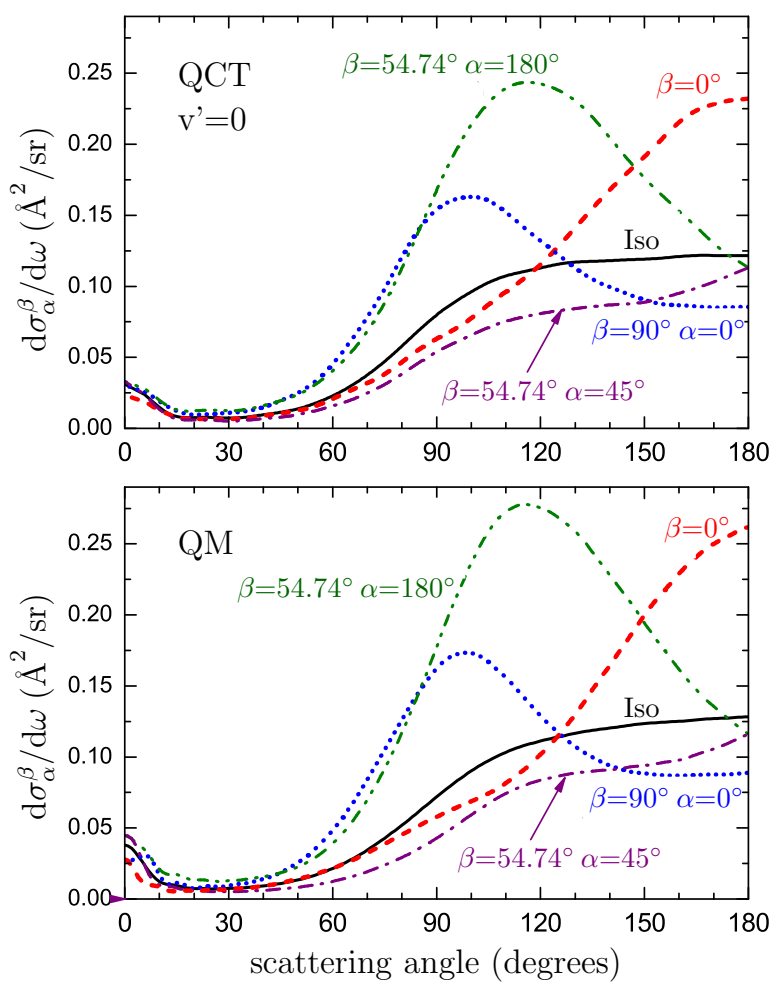

Fig. 4.11.: Differential cross section, summed over product rotational states, of reaction (4.62) at $E_{\text {coll }}=1.306 \mathrm{eV}$ and for different $\mathrm{D}_{2}$ alignment directions. Top panel shows quasiclassical results, bottom panel quantum mechanical ones.

one that is largely responsible for the polarization effects (the $s_{0}^{\{4\}}$, whose values were not presented before, is only slightly different from zero for most of the $j^{\prime}$ levels). As shown in Fig. 4.7, this PP is quite negative for $j^{\prime} \leq 5$ and quite positive for $j^{\prime} \geq 10$, changing sign around $j^{\prime}=8$. No surprise, then, that preparation of reactants with $a_{0}^{\{2\}}=-0.535,0$ or 0.267 (these are the quantum values of the extrinsic polarization moments corresponding to reactant alignment along $\beta=0^{\circ}, 54.74^{\circ}$ or $90^{\circ}$, respectively) lead to increasingly hot product rotational state distributions.

The effect of reactant polarization on differential cross sections, which requires to also consider specific values for the azimuthal angle $\alpha$, is now analysed. This further increases the stereospecificity of the experiment and can have a dramatic effect on the ability to control the system reactivity.

Fig. 4.11 shows quasiclassical (top) and quantum (bottom) differential cross sections 
for reactions leading to $\operatorname{HD}\left(v^{\prime}=0, j^{\prime}=\right.$ all $)$ at $E_{\text {coll }}=1.306 \mathrm{eV}$, considering selected combinations of values for $\beta$ and $\alpha$ as well as reactions without reactant polarization (labelled as "iso" in the picture). Given the level of detail of the property being considered, the agreement between quantum and QCT data is indeed remarkable, as is the variety of shapes one can obtain for the angular distributions by varying the direction along which the reactant molecule is aligned (i.e., by selecting specific values for $\beta$ and $\alpha$ ).

Inspection of Fig. 4.11 shows that head-on collisions (those favored by $\beta=0^{\circ}$ ) lead to an angular distribution that, compared to the one obtained in the isotropic case (that is, when the collision involves unpolarized reactants), is more focused on the backward scattering region. On the other hand, side-on collisions (those favored by $\beta=90^{\circ}$ ) enhance sideways scattering, while alignment along the magic angle $\left(\beta=54.74^{\circ}\right)$ leads to an intermediate result.

Also quite evident on Fig. 4.11 is the importance of the azimuthal angle $\alpha$, whose value can have a dramatic effect on the observed results. This is illustrated by the curves obtained with $\beta=54.74^{\circ}, \alpha=45^{\circ}$ or with $\beta=54.74^{\circ}, \alpha=180^{\circ}$ : while the former polarization direction leads to a DCS that has a similar shape to but is less intense than the DCS obtained with isotropic reactants, the latter leads to a very significant enhancement of the DCS, which is particularly pronounced near $\theta=115^{\circ}$. Note that $\alpha=0^{\circ}$ and $\alpha=180^{\circ}$ both correspond to situations in which the internuclear axis is on or near the scattering plane containing $\mathbf{k}$ and $\mathbf{k}^{\prime}$, but that the respective collision geometries differ, with the $\mathrm{D}_{2}$ interatomic axis being tilted along the quadrants of the scattering frame where the $r_{x} r_{z}$ product is either positive or negative (see Fig. 4.4). Values of $\alpha$ in the $45^{\circ} \leq \alpha \leq 135^{\circ}$ range, on the other hand, indicate predominance of collisions in which the $\mathrm{D}_{2}$ interatomic axis is close to perpendicular to the scattering plane. The fact that a large increase in the DCS is observed for $\alpha=180^{\circ}$ indicates that the reaction may be predominantly coplanar; i.e., the scattering plane and that containing the three atoms remain coincident in the course of the reaction (again, this will be more exhaustively discussed in next Chapter).

We present further illustration of the importance of the azimuthal angle $\alpha$ for the observed differential cross section on Fig. 4.12. It contains quantum state-to-state results for reactions leading to $\operatorname{HD}\left(v^{\prime}=0, j^{\prime}=0\right)$ scattering in the backward (top) and forward (bottom) scattering regions (the DCS of such reactions in the $30^{\circ} \leq \theta \leq 120^{\circ}$ region is invariably very small); the combinations of $\beta$ and $\alpha$ values are the same ones used in Fig. 4.11. Besides confirming the sensitivity of the product angular distribution to the value of the azimuthal angle $\alpha$, Fig. 4.12 shows that the variation 

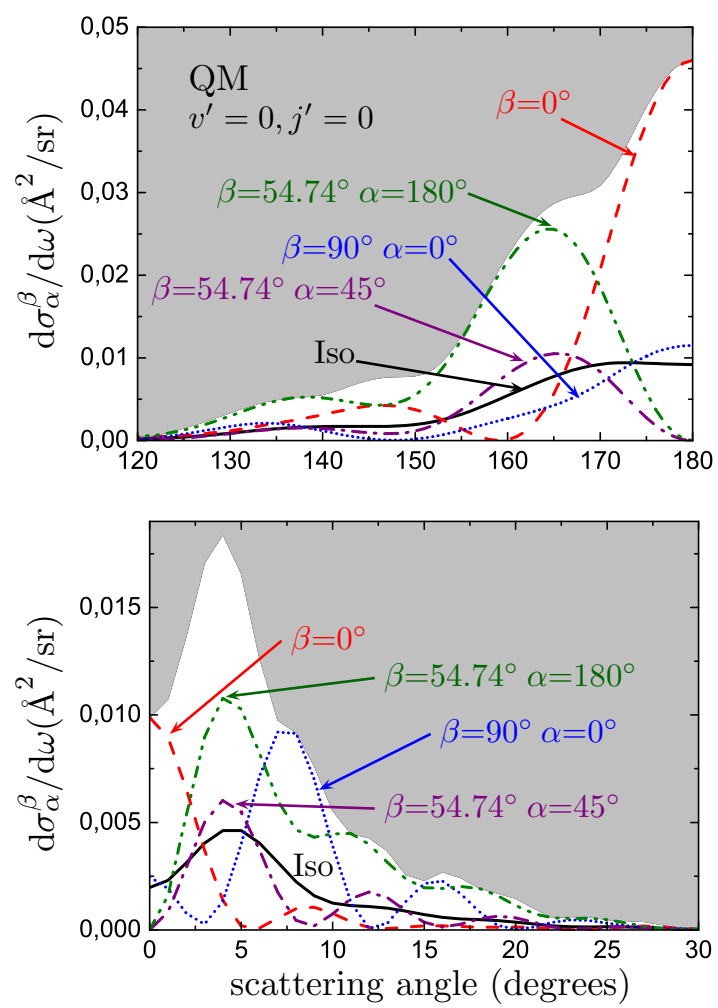

Fig. 4.12.: Quantum differential cross section of reaction (4.63) at $E_{\text {coll }}=1.306 \mathrm{eV}$ and for different $\mathrm{D}_{2}$ alignment directions. Top and bottom panel show backward and forward scattering region, respectively. The border between the white and gray areas represents the maximum possible values of $d \sigma_{\alpha}^{\beta} / d \omega$. The minimum possible values lie at the horizontal, $d \sigma_{\alpha}^{\beta} / d \omega=0$ axis. Minimum and maximum values were obtained with the min-maximization procedure described in Sec. 4.7.3.

can be significant even within strikingly small scattering angle intervals (something that, from the point of view of intrinsic properties, was illustrated by the strongly contrasting stereodynamical portraits of Fig. 4.6). Let us compare, for instance, $\beta=0^{\circ}$ to $\beta=54.74^{\circ}, \alpha=180^{\circ}$ : the first polarization direction leads to DCS maxima at $\theta=0^{\circ}$ and $\theta=180^{\circ}$ and to scattering nodes near $\theta=6^{\circ}$ and $\theta=160^{\circ}$, while the second polarization direction leads a DCS with scattering nodes where the first had maxima and with local maxima where the first had nodes. It is truly remarkable that such contrasts can be observed within such small scattering angle intervals.

Another way of visualizing the effect of the $\mathrm{D}_{2}$ alignment direction on the reaction we are considering here is by using scattering angle-recoil velocity polar maps such 


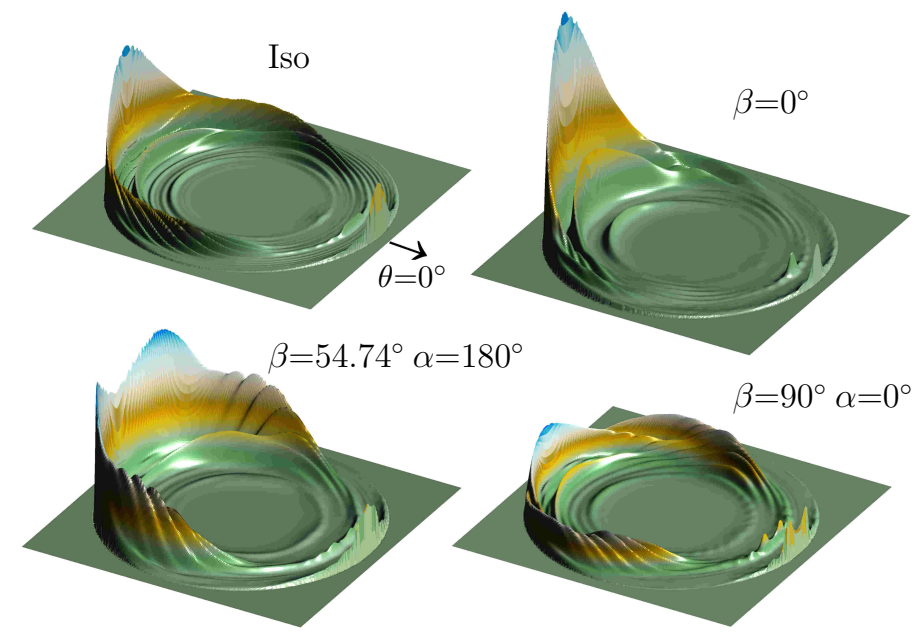

Fig. 4.13.: Quantum triple (angle-velocity) differential cross section of the $\mathrm{H}+\mathrm{D}_{2}(v=$ $0, j=2)$ reaction at $E_{\text {coll }}=1.306 \mathrm{eV}$ and for different $\mathrm{D}_{2}$ alignment directions.

as the ones on Fig. 4.13, which were plotted using quantum data obtained with $E_{\text {coll }}=1.306 \mathrm{eV}$. These polar maps show the value of the DCS over a plane in which the polar angle represents the scattering angle $\theta$, while the radial distance to the center is a measure of the product recoil energy (the larger the distance the larger the recoil energy and the smaller the internal energy of the products. The outer ring corresponds to $v^{\prime}=0, j^{\prime}=0$, and $v^{\prime}$ and $j^{\prime}$ increase towards the center). Previous observations are also visible in these plots: collinear collisions $\left(\mathrm{D}_{2}\right.$ alignment along $\beta=0^{\circ}$ ) lead to a pronounced enhancement of backward scattering with regard to the isotropic case, while side-on collisions $\left(45^{\circ} \leq \beta \leq 135^{\circ}\right)$ lead to an enhancement of sideways scattering, with the shapes and magnitudes of the polar maps also depending on whether the side-on collisions are coplanar or not. It becomes also clear from these representations that side-on collisions give rise to an appreciable rotational excitation especially manifest in the sideways scattering.

A further example of the effect of the $\mathrm{D}_{2}$ alignment on the reactivity can be obtained as follows: suppose that each of the previous polar maps is integrated over the scattering angle and the recoil velocity (this last integration is equivalent to sum over all the products states). The resulting quantity, the total special ICS, obviously depends on both $\beta$ and $\alpha$. Note that in this case the position of the scattering plane is well defined with respect to the direction of the polarization vector of the excitation laser in spite of the integration over the scattering angle $\theta$. The results obtained as a 


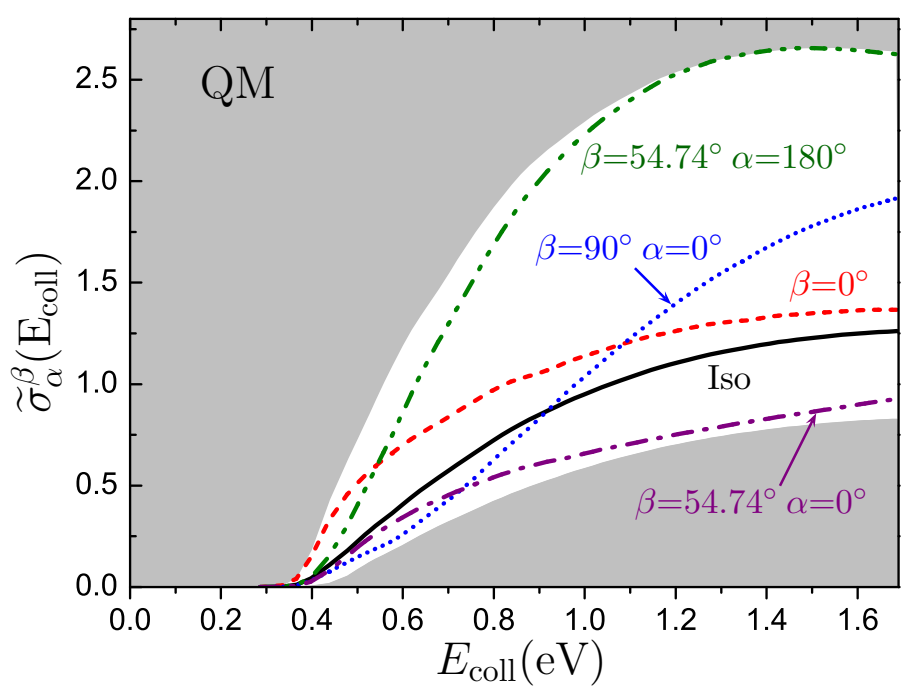

Fig. 4.14.: Quantum special ICS as a function of the $\mathrm{H}+\mathrm{D}_{2}(v=0, j=2)$ collision energy for different $\mathrm{D}_{2}$ alignment directions specified by the values of $\beta$ and $\alpha$. The borders between the white and gray areas represent the maximum and minimum possible values of $\tilde{\sigma}_{\alpha}^{\beta}$, and were obtained with the minmaximization procedure described in Sec. 4.7.3.

function of the collision energy are represented in Fig. 4.14 for different combinations of $\beta$ and $\alpha$. As will be explained in Sec. 4.7.3, the magnitude of the special ICS is bounded within the range indicated by the white area of the figure for a laboratory preparation of $|j=2, m=0\rangle$. At low collision energies the highest and lowest values of $\tilde{\sigma}_{\alpha}^{\beta}$ are obtained with $\beta=0^{\circ}$ and with $\beta=90^{\circ}, \alpha=0^{\circ}$, respectively. However, with increasing $E_{\text {coll }}$, the alignments that maximize and minimize the special ICS tend to be those with $\beta$ equal to the magic angle and $\alpha=180^{\circ}$ and $\alpha=0^{\circ}$, respectively. This indicates that side-on attack with the internuclear axis in the scattering plane can lead to maximal as well as minimal values for the special ICS, depending on the value of $\alpha$. This azimuthal angle is thus shown to be a very relevant stereodynamical parameter.

\subsubsection{The min-max approach: theoretical control limits}

The results just presented show that $\mathrm{D}_{2}$ polarization, and $\mathrm{D}_{2}$ alignment in particular, can have a dramatic effect on the outcome of the $\mathrm{H}+\mathrm{D}_{2}$ collision. By selecting specific directions for the $\mathrm{D}_{2}$ alignment, starkly contrasting reactive cross sections as well as starkly contrasting product state distributions have been obtained. 
The question that follows is this: is it possible to improve those results? Can one make the contrasts even starker? If one wants to increase or decrease the reactive cross sections, how far can one go? This is where the min-max approach steps in.

In other words, the question asked in the previous paragraph was, "can one determine the (extrinsic) reactant polarization moments that lead to minimal and maximal reactive cross sections?" The answer is that this is not only possible, but (theoretically at least) rather straightforward. All one has to do is, having determined intrinsic PPs and PDDCSs, to apply standard computational minimization/maximization techniques [69] using the cross section formulae (In the most general case, these are Eqs. (4.20), (4.23) and (4.25) and the details of the calculation were described in Section 4.4.4. If the goal is to determine what is achievable with the experimental setup described above, the required formulae are those of Eqs. (4.56-4.58)).

Results from two "min-max" calculations applied to the cross sections corresponding to the proposed experiment were shown in Figs. 4.9 and 4.14. The goal of these two examples was the determination of the direction of $\mathrm{D}_{2}(v=0, j=2)$ alignment that would lead to the largest or smallest possible values for the total ICS (Fig. 4.9) and for the total special ICS (Fig. 4.14).

In the case of the total ICSs of Fig. 4.9 the only adjustable parameter is the angle $\beta$, see Eq. (4.57). Its determination has lead to the minimal and maximal ICSs depicted in Fig. 4.9 as the borders between the experimentally accessible (white) and experimentally forbidden (gray) regions for $\sigma^{\beta}$. Fig. 4.15 further illustrates the results obtained, showing on its left panel the $\beta$ values leading to minimal and maximal ICSs at the collision energies considered; note that as the azimuthal angle $\alpha$ plays no role here (it is averaged out, as discussed in Sec. 4.4.2), one does not need to consider $\beta$ values outside the $0 \leq \beta \leq 90^{\circ}$ range.

Fig. 4.15 shows that the $\beta$ values that maximize $\sigma^{\beta}$ are invariably close to zero. This explains why the $\beta=0^{\circ}$ curve is invariably at or very close to the upper limit of the allowed $\sigma^{\beta}$ region in Fig. 4.9, and is further evidence that collinear $\mathrm{H}+\mathrm{D}_{2}$ collisions lead to an increased reactivity. As for the $\beta$ values that minimize $\sigma^{\beta}$, they are invariably close to $\beta=90^{\circ}$. This explains why the $\beta=90^{\circ}$ curve is invariably at or very close to the lower limit of the allowed $\sigma^{\beta}$ region in Fig. 4.9, and complements the maximization information, showing that side-on $\mathrm{H}+\mathrm{D}_{2}$ collisions lead to a decreased reactivity.

Min-maximization of the special ICS of Fig.4.14 involves two adjustable parameters ( $\beta$ and $\alpha$ ) rather than only one, see Eq. (4.58). Their determination has lead to the minimal and maximal special ICSs depicted in Fig. 4.14 as the borders between the experimentally allowed (white) and experimentally forbidden (gray) regions for $\tilde{\sigma}_{\alpha}^{\beta}$. 


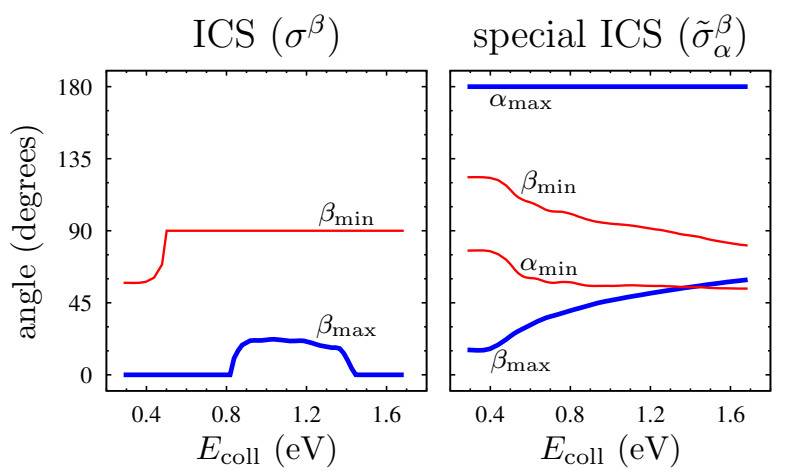

Fig. 4.15.: Values of the angles specifying the $\mathrm{D}_{2}(v=0, j=2)$ alignment directions leading to maximal or minimal total ICSs and total special ICSs according to QM. The maximal and minimal ICSs are shown on Fig. 4.9, and the maximal and minimal special ICSs on Fig. 4.14. In either case, they appear in those figures as the borders between the theoretically allowed (white) and theoretically forbidden (gray) regions.

Fig. 4.15 again further illustrates the results obtained, showing on its right panel the $\beta$ and $\alpha$ values leading to minimal and maximal special ICSs at the collision energies considered.

Fig. 4.15 shows that the $\beta$ and $\alpha$ values that maximize $\tilde{\sigma}_{\alpha}^{\beta}$ satisfy $\beta<90^{\circ}, \alpha=180^{\circ}$ : reactivity is enhanced by coplanar collisions in which the $\mathrm{D}_{2}$ interatomic axis is tilted towards the incoming $\mathrm{H}$ atom rather than away from it. It also shows that, when a more detailed analysis is carried out, one finds that the best collision geometry is actually not collinear (as far as reactivity is concerned). Indeed, none of the $\tilde{\sigma}_{\alpha}^{\beta}$ maximizations has lead to zero as the optimum $\beta$ value. Instead, it has been found that this optimum value steadily increases from $\beta \approx 15^{\circ}$ near the reaction threshold to $\beta \approx 60^{\circ}$ at the highest collision energies considered. At the high collision energies the optimum collision geometry is not even approximately collinear as suggested by the $\sigma^{\beta}$ values, but rather coplanar and approximately side-on. This explains why the $\beta=0^{\circ}$ curve is close to the upper limit of the allowed $\tilde{\sigma}_{\alpha}^{\beta}$ region in Fig. 4.14 only at very low collision energies, and also why at higher collision energies it is coplanar, magic-angle collisions that lead to special ICSs approaching their maximum possible values. As shown by Fig. 4.14, selection of the azimuthal angle allows one to double the system reactivity.

As for the $\beta$ and $\alpha$ values that minimize $\tilde{\sigma}_{\alpha}^{\beta}$, they invariably indicate that side-on collisions in which the $\mathrm{D}_{2}$ axis is perpendicular to the scattering plane are those that most reduce reactivity. Consideration of Fig. 4.14, however, leads to an interesting 
observation: coplanar, magic-angle collisions in which the $\mathrm{D}_{2}$ interatomic axis is tilted the "wrong" way (away from the incoming $\mathrm{H}$ atom rather than towards it) can lead to a similar reactivity reduction, in particular at the higher collision energies at which the "right" coplanar, magic-angle collisions lead to almost maximum $\tilde{\sigma}_{\alpha}^{\beta}$ values.

The Section will be closed with three remarks about application of the min-max approach. The first is that, although the reported min-max calculations have been constrained (the scattering-frame extrinsic reactant polarization moments were obtained by rotation of laboratory-frame moments, and the values of these were fixed), they do not have to be limited in this way: the values of the extrinsic reactants polarization moments, the $a_{q}^{(k)}$ 's, can be chosen freely. Unconstrained min-max calculations were presented in Section 4.4.4. They are not not significantly harder than the constrained ones, and when performed, they can eventually lead to significantly larger maximal cross sections and to virtually zero minimal cross sections.

Fig. 4.16 serves to illustrate the differences between both kinds of calculations. It presents the quantum mechanical enhancement and suppression numbers ${ }^{9}$ for the total integral cross section of reaction $\mathrm{H}+\mathrm{D}_{2}(v=0, j=2)$. Continuous lines correspond to unconstrained calculations and dashed lines to constrained calculations. While suppression numbers derived from unconstrained calculations (red continuous line) always "improve" their constrained counterparts (dashed red line), enhancement numbers for unconstrained (blue continuous line) and constrained (dashed blue line) calculations are almost identical in this case. The analysis of the Figure shows two additional features of the reaction: (i) the reactants polarisation is more effective for hindering than for spurring the reaction (the largest value of the enhancement number is 1.5, the smallest of the suppression number is around -3.5) and (ii) the achievable control dramatically decreases as the collision energy increases. Anticipating results included in the next Chapter, this last feature can be interpreted in terms of access to the transition state region of the PES. For the BKMP2 PES, such transition state has a linear geometry and turns out to be quite stiff, that is, small deviations from the linear geometry give rise to noticeable increases of energy. While at low collision energy the access to the TS region of the PES is limited to collisions whose geometry is close to collinear, an increasing number of geometries allow for reaching the PES region as the collision energy rises and, in consequence, a lower improvement is expected from suitably preparing the reactants prior to reaction.

The second remark is that, although only min-max results for integral cross sections have been discussed, the approach can also be applied to other reaction properties, e.g. differential cross sections and product state distributions. Indeed, Fig. 4.12 shows

\footnotetext{
${ }^{9}$ Remember that these numbers must be compressed in the interval $[-\infty, \ln (2 j+1)]$
} 


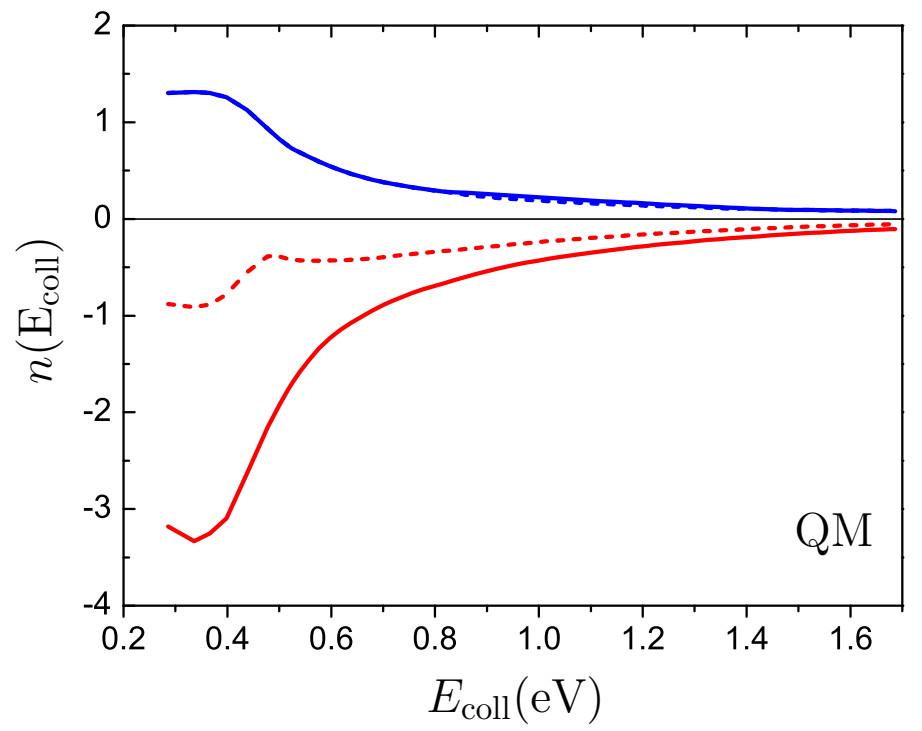

Fig. 4.16.: Quantum mechanical enhancement (positive quantities) and suppression (negative quantities) numbers corresponding to maximization and minimization of the total integral cross section for reaction $\mathrm{H}+\mathrm{D}_{2}(v=0, j=2)$. Continuous lines correspond to unconstrained calculations while dashed lines hold for constrained calculations (their values were obtained with the data presented in Fig. 4.9).

min-max results for a differential cross section: the maximization results are shown as the border between the theoretically allowed (white) and theoretically forbidden (gray) regions, while the minimization results (which have lead to a vanishing DCS for every scattering angle) lie along the horizontal, $d \sigma_{\alpha}^{\beta} / d \omega=0$ axis.

The final remark is that in general the results from min-max calculations will be better represented by extrinsic stereodynamical portraits (or, equivalently, by a complete set of extrinsic polarization moments) than by a single direction along which reactants are to be oriented or aligned. In the case of the constrained results presented above the distinction was unnecessary, as the calculations were done with the experimental setup described in Sec. 4.6 in mind, and the $\beta$ and $\alpha$ values uniquely determined the extrinsic reactants portraits and polarization moments, see Fig. 4.4 and Eq. (4.54). However, when unconstrained min-maximizations are performed this is certainly not the case and the set of polarisation moments can not be studied in terms of an smaller group of parameters. In general, it is comparison between the actual (extrinsic) reactants polarization portraits and their intrinsic counterparts that will allow 
one to obtain the full picture. Production of pure states can be used as an example. If the DCS of reaction (4.63) at $E_{\text {coll }}=1.306 \mathrm{eV}$ and $\theta=4^{\circ}$ is studied, the discussion of Sec. 4.4 .4 anticipates what the result of an unconstrained maximization must be: the extrinsic portraits corresponding to the reactants polarisation that maximize the reactivity must be identical to the intrinsic portraits shown of the top row of Fig. 4.6 (this is because in the case of production of pure states it is possible to create a pure reactant polarization state that, in terms of the metaphor introduced earlier, gives to the reaction exactly what it wants). Considering the stereodynamical portraits on Fig. 4.6 one can see that they are not defined simply by a particular direction in space, as their shapes can also change. In order to fully understand the stereochemistry of a reactive collision, one must consider the full picture (directions and shapes of the spatial distributions of molecular axes and rotational angular momenta) rather than a single spatial direction. 


\section{Mechanism of the $\mathbf{H}+\mathrm{D}_{2}$ reactive collisions}

Examples in Sec. 4.7 were divided into three different categories corresponding to different (and complementary) ways of analysing the reaction stereodynamics through the reactants polarisation: (i) "Intrinsic approach" (Sec. 4.7.1), (ii) "Practical approach" (Sec. 4.7.2) and (iii) "The min-max approach" (Sec. 4.7.3), where the former was specially suitable for the reaction mechanism analysis and the other two for the study of the control possibilities. Although those examples paid more attention to the control problem than to unravel the reaction mechanism, they allowed for extracting some information regarding the way in which $\mathrm{H}+\mathrm{D}_{2}$ reactive collisions happen:

- The internuclear axis of the $\mathrm{D}_{2}$ molecule is preferably placed on the scattering plane, suggesting that the reaction is coplanar.

- There is a relation between the collision geometry and the rotational excitation of the products (head-on (side-on) reactive collisions take to little (large) rotational excitation of the products).

- The intrinsic PDDCSs in the forward region display very fast oscillations that could be attributed to an interference effect.

Confirmation and explanation of these three points, together with the unveiling of new reaction features, is provided by this Chapter contents, devoted to rationalisation and understanding of $\mathrm{H}+\mathrm{D}_{2}$ reaction mechanism. The strategy chosen to tackle the problem follows that sketched in Sec. 4.7.1 the reaction mechanism is studied by means of the intrinsic polarisation moments (they contain the reaction directional preferences). Such moments were worked out from the scattering matrices in the helicity representation ${ }^{1}$ (only quantum mechanical results are presented) for

- the total energy range $0.50-1.75 \mathrm{eV}$ and

\footnotetext{
${ }^{1}$ The calculation of the $\mathrm{S}$ matrix was performed by Dr. Brian Kendrick with previously reported [112] time independent calculation on the BKMP2 surface [11]
} 
- the initial states $\mathrm{D}_{2}(v=0-1, j=1-2)$.

As no major distinctions were found between the four $\mathrm{D}_{2}$ states, the presentation is restricted to results for the

$$
\mathrm{H}+\mathrm{D}_{2}(v=0, j=2) \rightarrow \mathrm{HD}\left(v^{\prime}, j^{\prime}\right)+\mathrm{D}
$$

reaction, for which the range of collision energies is $0.29-1.54 \mathrm{eV}$. The reader should bear in mind, however, that the conclusions are valid for all four rovibrational states.

The Chapter begins with a brief summary (Sec. 5.1) of some significative findings about the reaction mechanism presented in earliest works. Next, the intrinsic moments are analysed going from less detailed to more detailed quantities: it starts from total PPs (Sec. 5.2), goes through state-to-state PPs (Sec. 5.3) and total PDDCSs (Sec. 5.4) and finishes with the consideration of the state-to-state PDDCSs (Sec. 5.5), which offer the more exhaustive description of the reaction mechanism.

The two notations introduced in Chapter 4 for representing the intrinsic moments will be combined: the real PDDCSs (PPs) will be represented either as $\operatorname{PDDCS}\{k, q \pm\}$ $(\mathrm{PP}\{k, q \pm\})$ or as $S_{q \pm}^{\{k\}}(\theta)\left(s_{q \pm}^{\{k\}}\right)$.

\subsection{Direct and delayed mechanisms}

At this range of energies, two basic types of reactive collisions have been invoked to explain the $\mathrm{H}+\mathrm{D}_{2}$ reaction mechanism [110]:

- The first, which is held to proceed without formation of a short-lived reaction complex, has been dubbed the "direct" mechanism. To a first approximation, this mechanism is due to low to medium impact parameter collisions that lead to backward or sideways product scattering [67, 96, 110, 114, 117].

- In contrast, the second mechanism proposed for the $\mathrm{H}+\mathrm{D}_{2}$ reaction does involve formation of a short-lived reaction complex, which in turn leads to a time delay in product formation. It has been dubbed the "delayed" mechanism. This mechanism is largely due to high impact parameter collisions that lead to forward scattering of HD products in low rotational states $67,96,110,114,117$.

As it will be shown below, the stereodynamical analysis of the problem allows for further characterisation of the direct and delayed mechanisms (although our study is time-independent, we still refer to the two mechanisms by their established names).

Characterisation of the direct mechanism is relatively easy. This is in part because the direct mechanism is usually dominant, and in part because its stereodynamics is 
not only distinctive but also relatively well-behaved, changing smoothly with energy and scattering angle.

Characterisation of the delayed mechanism, on the other hand, is more challenging. This is so because the delayed mechanism is rarely dominant, but also because its stereodynamics can oscillate strongly, in particular with scattering angle (an analysis of the causes of these oscillations will be presented).

The differences just mentioned should be kept in mind throughout this Chapter. As our presentation proceeds from general aspects (total PPs) to specific details (stateto-state PDDCSs) of the $\mathrm{H}+\mathrm{D}_{2}$ reaction stereodynamics, the results we will encounter first largely reflect the direct mechanism, with only small hints about the delayed one. Only when the state-to-state PDDCSs are considered, it will be feasible to analyse in depth the delayed mechanism.

\subsection{Total integral results}

In this section we consider total PPs and the associated stereodynamical portraits. The question with which this section is concerned is this: what reactant polarisation does lead to reaction?

In order to completely characterise (quantum-mechanically) the polarisation of a $j=2$ state, one needs to determine the values of twenty-four real polarisation moments: the ones whose rank satisfies $1 \leq k \leq 4$. Symmetry constraints (see Eqs. 4.8) imply that twelve of them must vanish, which leaves us with twelve real polarisation moments to consider.

Fig. 5.1 shows how the values of four of the twelve nonvanishing total PPs (those with $k=1-2$ ) change with collision energy.

What the Figure is telling about the results is that most total PPs show little variation with collision energy (notice that in Fig. 5.1 the ranges of the vertical axes match the allowed ranges of the PPs). The major exception is $\operatorname{PP}\{2,0\}$, whose value changes quite noticeably: it is close to the negative limit at low collision energies, and close to zero at high collision energies.

Polarisation moments with $k=2$ and $q=0$ quantify alignment with regard to the $z$ axis, which in our case corresponds to $\mathbf{k}$, the reactant-approach direction: negative values imply a preference for $\mathbf{j} \perp \mathbf{k}$ and $\mathbf{r} \| \mathbf{k}$. This information, along with the results in Fig. 5.1, leads to the following conclusion: as the collision energy increases, the major change in the intrinsic reactants polarisation must be a loss of preference for head-on, $\mathbf{r} \| \mathbf{k}$ collisions.

It is necessary to recall that, in order to figure out how exactly the intrinsic $\mathrm{D}_{2}(v=$ 

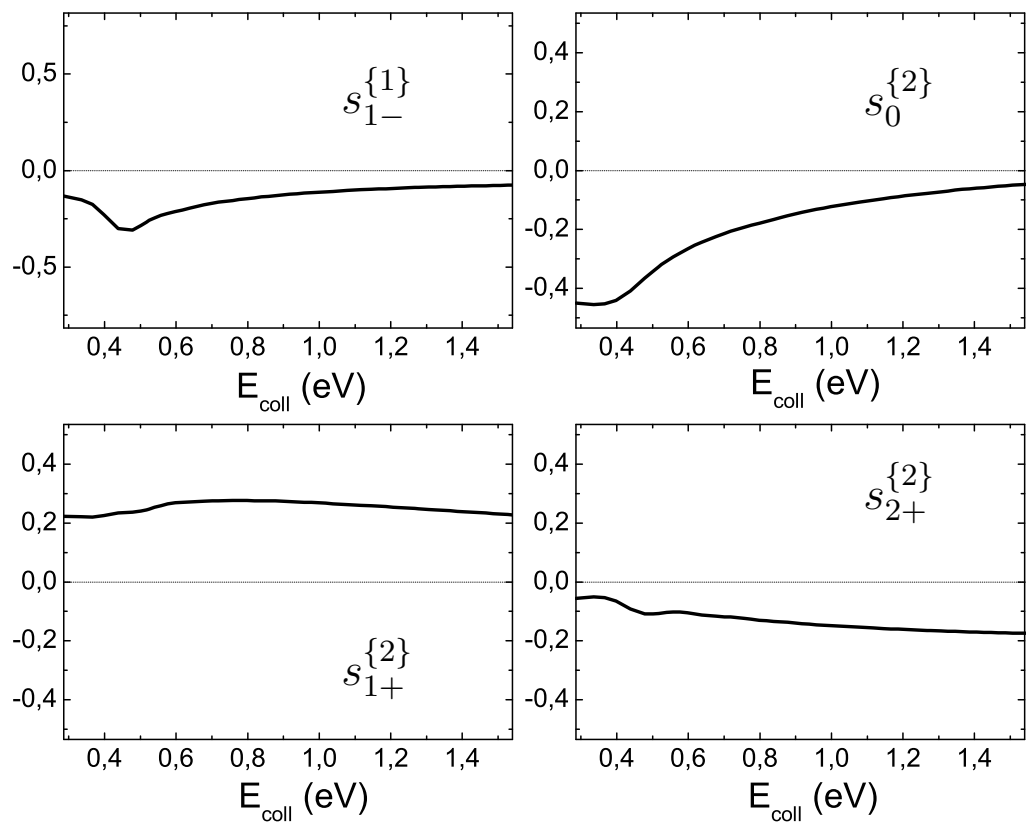

Fig. 5.1.: Dependence of nonvanishing total polarisation parameters (total, averaged over final states PPs) of rank $k=1-2$ on collision energy. The ranges of the vertical axes match the allowed ranges of these PPs for $j=2$.

$0, j=2$ ) polarisation changes with collision energy, one must simultaneously consider the directional meanings and value variations of all PPs: not only the four shown on Fig. 5.1, but also the eight others. This, as already mentioned, is a task which needs the employment of the stereodynamical portraits: direct visualisation of the intrinsic $\mathrm{D}_{2}$ polarisation is made possible by inspection of Fig. 5.2 , which shows $\mathrm{D}_{2}(v=0, j=2)$ molecular axis and rotational stereodynamical portraits ${ }^{2}$ at low, intermediate and high collision energy values (respectively, $0.501,0.998$ and $1.506 \mathrm{eV}$ ).

These portraits neatly summarise the information contained in figure 5.1 and give an straightforward answer to the question in the opening paragraph of this Section, thus allowing for immediate insight into the overall reaction mechanism. The rotational portrait corresponding to the lowest energy (right upper panel) displays a clear orientation along the $-y$ axis (that is, a preference for $\mathrm{D}_{2}$ rotation parallel to the scattering plane and in a clockwise sense when seen from the $y>0$ side of the scattering plane) and is approximately perpendicular to the $z$ axis. As the energy increases

\footnotetext{
${ }^{2}$ For the sake of clarity, the axis will be henceforth displaced from the center of the portraits as in Chapter 4
} 

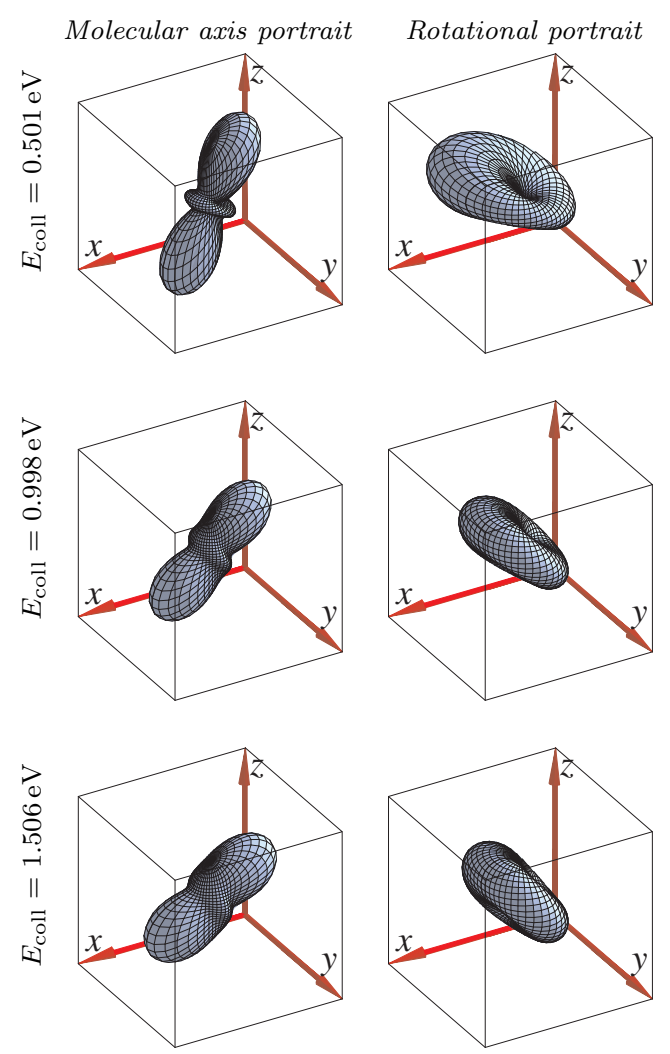

Fig. 5.2.: Molecular axis and rotational $\mathrm{D}_{2}(v=0, j=2)$ stereodynamical portraits prepared using total PPs at $E_{\text {coll }}=0.501,0.998$ and $1.506 \mathrm{eV}$. See Sec. 4.3 for a definition of the $x y z$ scattering frame.

that orientation and the alignment perpendicular to the $z$ axis decrease and, finally, almost disappears. Globally, the change in the values of the moments gives rise to a tilting with respect to the $z$ axis. This tilting is the most remarkable modification of the portraits and is related to the diminishing value of the $\operatorname{PP}\{2,0\}$ moment as the energy increases while the $\operatorname{PP}\{2,1+\}$ moment has a consistently positive value $(\mathbf{j}$ preferably aligned along $x+z$ instead of along $x-z$ ).

The molecular axis portraits (left column in figure 5.2), although based on the same polarisation moments as their rotational counterparts, provide us with an even more pictorial description, this time in terms of the internuclear axis distribution that yields the reaction. As mentioned above, these distributions are insensitive to the odd polarisation moments. For the lowest collision energy, the distribution giving rise to reaction clearly shows a preference for the $\mathrm{D}_{2}$ molecular axis to be approximately 


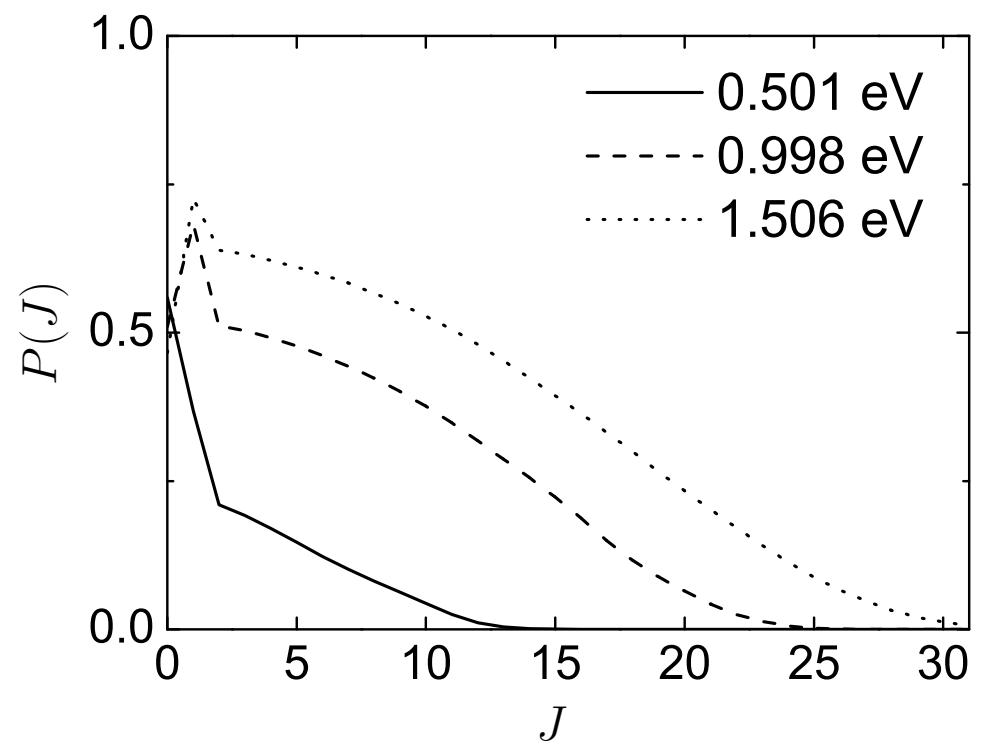

Fig. 5.3.: Reaction probability as a function of the total angular momentum quantum number $(J)$ for reaction $\mathrm{H}+\mathrm{D}_{2}(v=0, j=2) \rightarrow \mathrm{D}+\mathrm{HD}\left(v^{\prime}=\right.$ all, $j^{\prime}=$ all $)$ at $E_{\text {coll }}=0.501,0.998$ and $1.506 \mathrm{eV}$.

aligned along the relative velocity $\mathbf{k}$, with a light tilting so that the "first" D atom (the one closest to the $\mathrm{H}$ atom approaching the $\mathrm{D}_{2}$ molecule along the $z \equiv \mathbf{k}$ direction) lies towards the $x>0$ half of the $x z$ plane. As the collision energy increases it becomes apparent that the angle between the molecular axis portrait and $\mathbf{k}$ increases.

At this point it is already interesting to try to relate the shapes of the portraits to the global mechanism of the reaction into all final states at these collision energies. One of the main features revealed by these portraits is the fact that the internuclear axis tends to be aligned along a direction contained on the $x z$ plane, i.e., the scattering plane. The second aspect is that at low collisions energies the internuclear axis are much more aligned along the relative velocity than at high energies.

These results are consistent with a direct, rebound-type mechanism and can be rationalised by taking into account that the $\mathrm{H}+\mathrm{D}_{2}$ reaction is collinearly constrained and has a linear transition state [111. Let us consider what would happen when a collision takes place. The atom approaches the molecule from the region of negative $z$ values with a direction that is parallel to $z$ and a distance on the $x y$ plane from the center of the mass equal to the impact parameter. At low collision energies, when only small impact parameters contribute (see Fig. 5.3 ), the reaction is constrained to geometries close to that of the transition state. Small deviations from it (small tilts 
of the $\mathrm{D}_{2}$ interatomic axis, nonzero impact parameter), lead to product scattering in the plane containing the three atoms, and in the half of that plane flagged by the $\mathrm{H}$ and "first D" atoms. $\mathrm{D}_{2}$ rotation also plays a part in the selection of the half-plane containing $\mathbf{k}^{\prime}$; the direction of motion of the first $\mathrm{D}$ atom is roughly preserved upon transfer from the $\mathrm{D}_{2}$ reactant to the HD product.

As the collision energy increases, the $\mathrm{H}-\mathrm{D}-\mathrm{D}$ complex can take increasingly bent configurations. On one hand, this opens the door for contributions from higher impact parameters (see Fig. 5.3); on the other, this leads to the shift of preference (from $\mathbf{r}$ near $z$ to $\mathbf{r}$ near $x$ ) seen on Fig. 5.2 .

As for rotational orientation, at high collision energies its effect is seen to be minor compared to those associated with tilting of the $\mathrm{D}_{2}$ axis. While it may play a role in determining exactly what product state is scattered into what scattering angle (we will later show that it indeed does), it does not significantly alter the overall reaction probability.

\subsection{State-to-state integral results}

The state-to-state PPs and the associated stereodynamical portraits will be now considered. The question with which this Section is concerned is this: what reactant polarisation does lead to formation of products in a given state?

Analysis of the data has showed that, under the conditions considered in this Chapter, the stereodynamics of the $\mathrm{H}+\mathrm{D}_{2}$ reaction is largely insensitive to the HD product vibrational state, but quite sensitive to its rotational state. Furthermore, it was found that although the balance between the various stereodynamical effects does depend on the system's energy, their nature and relative contributions are best rationalised by consideration of results involving a large number of contributing product rotational states, that is, results obtained with a high collision energy and a low $v^{\prime}$ value.

Fig. 5.4 shows the integral cross section of the $\mathrm{H}+\mathrm{D}_{2}(v=0, j=2) \rightarrow \operatorname{HD}\left(v^{\prime}=\right.$ $\left.0, j^{\prime}\right)+\mathrm{D}$ reaction at $E_{\text {coll }}=1.506 \mathrm{eV}$ as a function of $j^{\prime}$, as well as molecular axis and rotational $\mathrm{D}_{2}$ stereodynamical portraits prepared using state-to-state PPs for selected $j^{\prime}$ values, namely $j^{\prime}=2,9$ and 13 . These rotational states have been chosen to clearly illustrate the effect of the rotational polarisation on the product's rotational excitation.

Exam of the $\mathrm{D}_{2}$ molecular axis portraits (those on the top of Fig. (5.4) shows (i) that all state-to-state reactions are favoured by placement of the $\mathrm{D}_{2}$ interatomic axis on the scattering plane, and (ii) that the extents of product rotational excitation and reactant axis tilt are correlated; reactions leading to low (high) $j^{\prime}$ values are favoured 


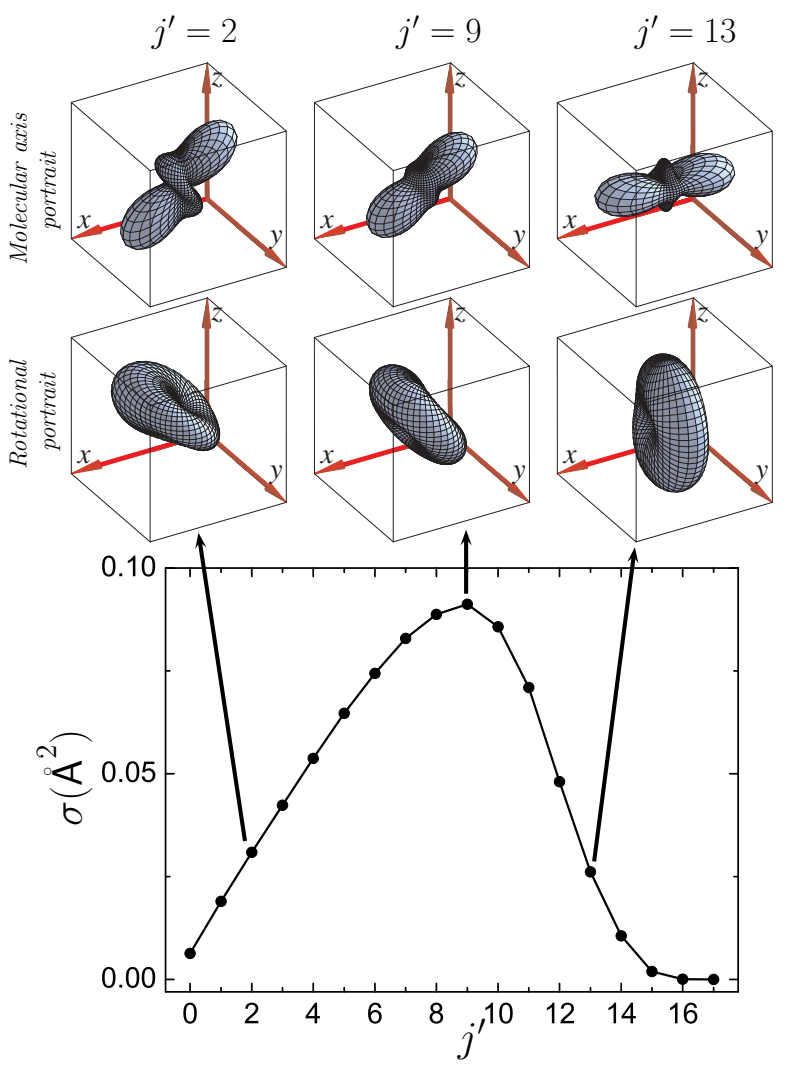

Fig. 5.4.: Integral cross section of the $\mathrm{H}+\mathrm{D}_{2}(v=0, j=2) \rightarrow \mathrm{HD}\left(v^{\prime}=0, j^{\prime}\right)+\mathrm{D}$ reaction at $E_{\text {coll }}=1.506 \mathrm{eV}$ as a function of $j^{\prime}$, and molecular axis and rotational $\mathrm{D}_{2}$ stereodynamical portraits prepared using state-to-state PPs for the same reaction and selected $j^{\prime}$ values.

by $\mathrm{D}_{2}$ polarisations in which the molecular axis is less (more) tilted.

These observations can be rationalised as those in the previous Section: by consideration of the extent of bending of the H-D-D complex around its linear minimumenergy geometry, and also of the impact parameters contributing to reaction. Less (more) $\mathrm{H}-\mathrm{D}-\mathrm{D}$ bending is associated with less (more) $\mathrm{D}_{2}$ axis tilting, smaller (larger) impact parameters ${ }^{3}$, and less (more) product rotational excitation. This latter, product rotational excitation effect, is associated with an HDD bending-to-HD rotation energy transfer that will be discussed in a moment.

Exam of the $\mathrm{D}_{2}$ rotational portraits (those on the middle of Fig. 5.4) reveals an

${ }^{3}$ The state-to-state probability functions $P(J)$ (not included here), show that less (more) rotationally excited products come from reactions characterised by smaller (larger) impact parameters 


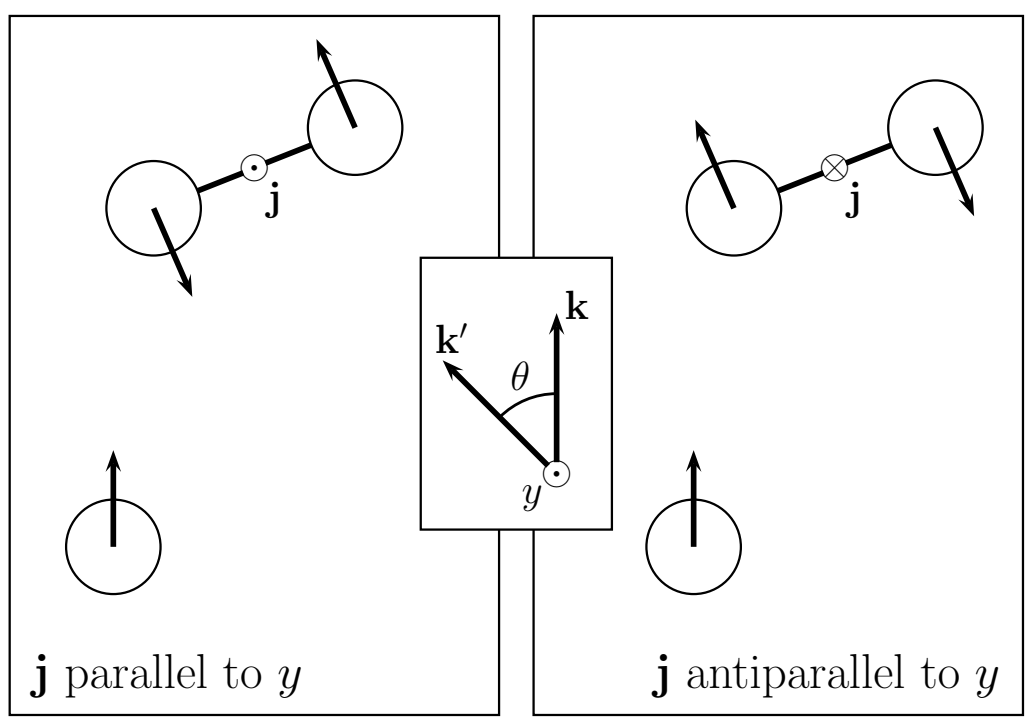

Fig. 5.5.: Cartoon illustrating the influence of $\mathrm{D}_{2}$ rotational orientation on HD rotational excitation. If $\mathbf{j}$ orientation is parallel (antiparallel) to $y \equiv \mathbf{k} \times \mathbf{k}^{\prime}$, then the $\mathrm{H}$ and $\mathrm{D}$ atoms collide while moving along roughly opposite (similar) directions. This leads to more (less) HDD bending vibration, which in turn leads to more (less) HD rotation.

additional feature: the extents of product rotational excitation and reactant rotational orientation are correlated; reactions leading to high (low) $j^{\prime}$ values are favoured by $\mathbf{j}$ orientation parallel (antiparallel) to the $y$ axis.

The cartoon shown on Fig. 5.5 provides a rationale for this effect: if $\mathbf{j}$ orientation is parallel (antiparallel) to $y \equiv \mathbf{k} \times \mathbf{k}^{\prime}$, then the $\mathrm{H}$ and $\mathrm{D}$ atoms collide while moving along roughly opposite (similar) directions. This leads to more (less) HDD bending vibration, which in turn leads to more (less) HD rotation.

Note also that our cartoon implicitly assumes that the reaction takes place with all molecular axes on the scattering plane. This assumption, is justified not only by our own results, but also by those from a previous, quantum and quasiclassical trajectory studies of the title reaction [118. It has been shown there a tendency for the orbital and rotational angular momenta to be either parallel or antiparallel to each other, a finding that corroborates the coplanarity assumption [118.

At this point, however, a note of caution is needed: Fig. 5.5 accounts for the dominant reaction mechanism, but not for all reaction mechanisms. As will be seen in Sec. 5.5, differential state-to-state results suggest that other, possibly quite different mechanisms, can also contribute to the reaction dynamics. In particular, a more 
detailed analysis reveals that the coplanarity condition is not always fulfilled.

A final remark is that Fig. 5.5 suggests that formation of HD in a high-lying rotational state should be associated with strong product rotational orientation: $\mathbf{j}^{\prime}$ should be strongly oriented along $-y$. Previous studies have found this to be indeed the case [25, 98 .

\subsection{Total differential results}

Thus far, only results integrated over scattering angles have been considered. That restriction is now lifted, and our attention turn to the investigation of the correlation between reactant polarisation and scattering angle. This will allow to distinguish between mechanisms associated with forward, sideways and backward scattering.

In this Section renormalised total PDDCSs and the associated stereodynamical portraits will be considered. The question with which this Section is concerned is this: what reactant polarisation does lead to scattering of products into a given scattering angle?

Fig. 5.6 shows the total DCS (top) and the renormalised total PDDCS $\{2,0\}$ (bottom) at low, intermediate and high values of the collision energy (respectively, 0.501, 0.998 and $1.506 \mathrm{eV})$.

The first observation concerns the range of scattering angles. At the lowest collision energy, the DCS is confined to the backward hemisphere; product scattering at $\theta<90^{\circ}$ is unlikely. As the collision energy increases, the situation changes somewhat, with scattering angles in the sideways and forward scattering regions becoming, if not dominant, at least not negligible. Note also the appearance of a clear peak in the forward scattering region of the DCS at $E_{\text {coll }}=1.506 \mathrm{eV}$.

As in the case of total integral results (Sec. 5.2 ), we have found that the polarisation moment giving most direct information about the major variations in the stereodynamics (here, with scattering angle as well as collision energy) is the one quantifying alignment with regard to the $z \equiv \mathbf{k}$ axis. In other words, the renormalised total $\operatorname{PDDCS}\{2,0\}$ in the bottom panel of Fig. 5.6.

At the lowest collision energy, the PDDCS $\{2,0\}$ is invariably negative, but the less so the further away from $180^{\circ}$ the scattering angle is. The negative values indicate a preference for $\mathbf{j} \perp \mathbf{k}$ and $\mathbf{r} \| \mathbf{k}$ that becomes less pronounced as one moves away from backward, $\theta=180^{\circ}$ scattering.

As the collision energy increases, in the backward $\left(120^{\circ} \leq \theta \leq 180^{\circ}\right)$ scattering region one finds a weakening of the preference for negative $\operatorname{PDDCS}\{2,0\}$ values. In the sideways and forward $\left(\theta \leq 120^{\circ}\right)$ scattering regions, the shift is from mildly 

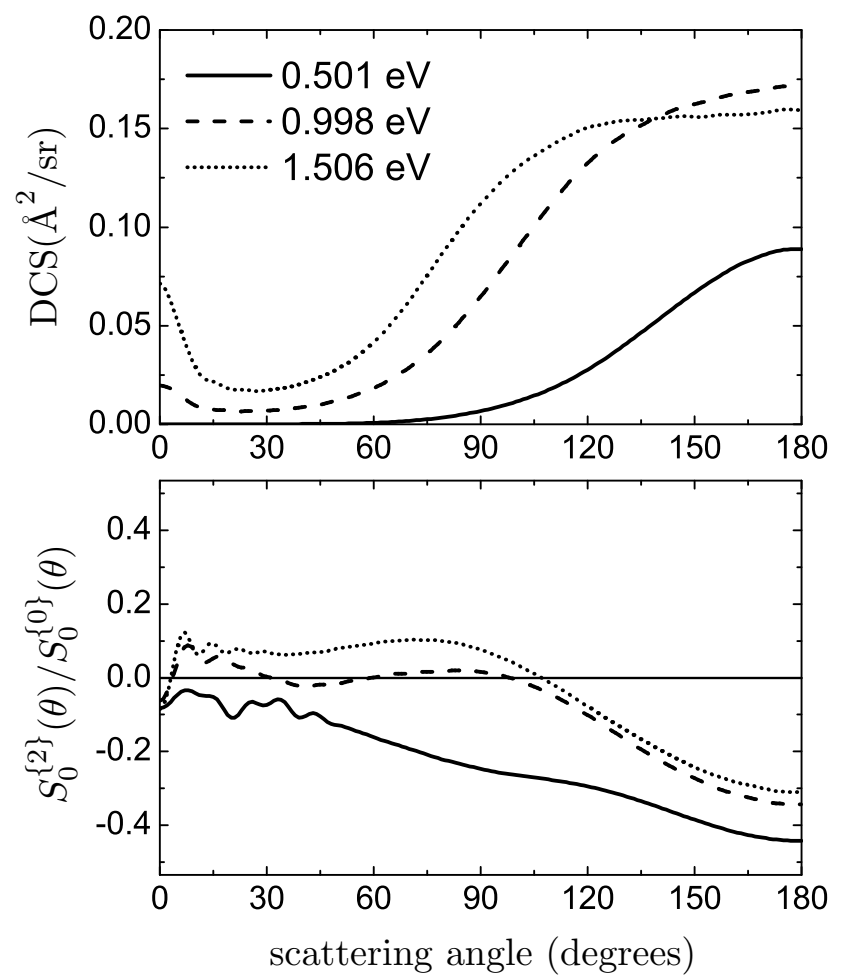

Fig. 5.6.: Total DCS (top) and renormalised total PDDCS $\{2,0\}$ (bottom) at $E_{\text {coll }}=$ $0.501,0.998$ and $1.506 \mathrm{eV}$. In the bottom panel, the range of the vertical axis matches the allowed range of the renormalised PDDCS $\{2,0\}$ for $j=2$.

negative to mildly positive $\operatorname{PDDCS}\{2,0\}$ values - a shift from mild preference for $\mathbf{j} \perp \mathbf{k}$ and $\mathbf{r} \| \mathbf{k}$ towards mild preference for $\mathbf{j} \| \mathbf{k}$ and $\mathbf{r} \perp \mathbf{k}$.

Although the discussion of PDDCS values could be taken further, it is now clear that stereodynamical portraits provide a much less cumbersome description of the problem. We therefore turn to those, selecting as before a case that allows for maximum clarity in the analysis of the various effects: the highest of the three energies in Fig. 55.6 $\left(E_{\text {coll }}=1.506 \mathrm{eV}\right)$, and scattering angles of 0,90 and $180^{\circ}$. The corresponding $\mathrm{D}_{2}$ molecular axis and rotational portraits are shown on Fig. 5.7.

Let us first examine the results for the two limiting values of the scattering angle, $\theta=0^{\circ}$ and $180^{\circ}$. At these angles, the shapes that stereodynamical portraits can possibly take are severely restricted. This is due to the initial and final relative velocity vectors being parallel or antiparallel: the scattering plane is undefined, and as a consequence all portraits must show cylindrical symmetry around the $\mathbf{k}$ axis (in 


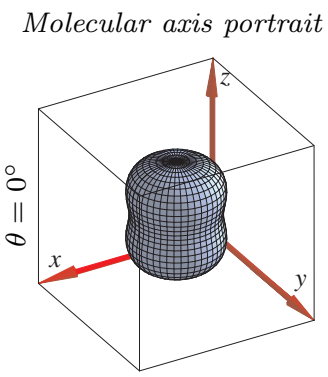

Rotational portrait
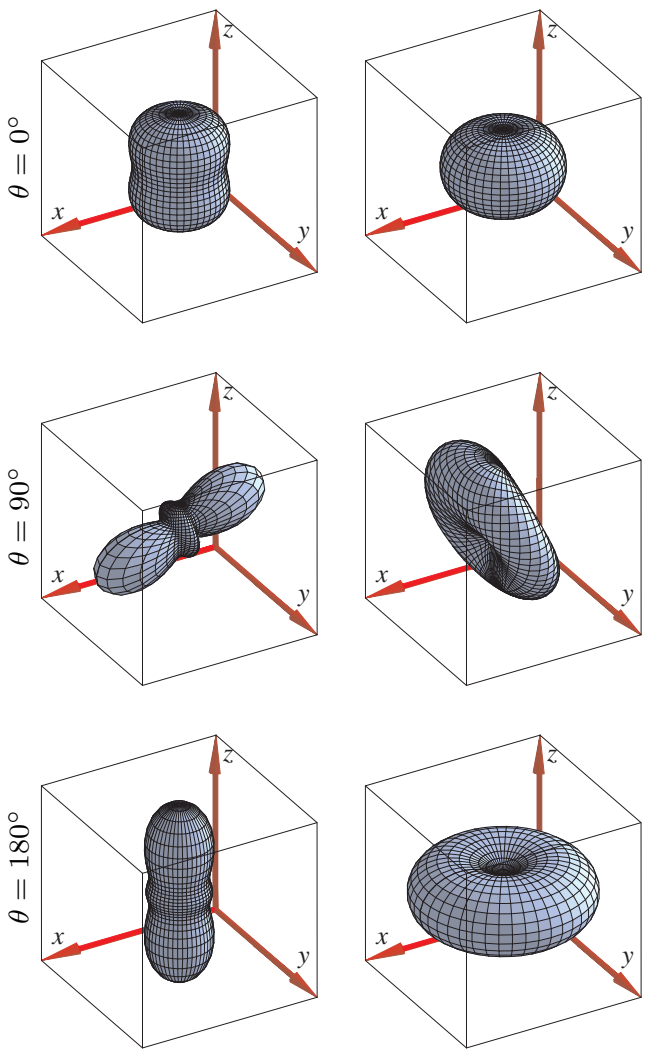

Fig. 5.7.: Molecular axis and rotational $\mathrm{D}_{2}(v=0, j=2)$ stereodynamical portraits prepared using renormalised total PDDCSs at $E_{\text {coll }}=1.506 \mathrm{eV}$ and for three different values of the scattering angle: $\theta=0,90$ and $180^{\circ}$.

other terms, the only PDDCSs whose value can change are those with $k=2$ or 4 and $q=0$; PDDCSs with $k$ odd or $q \neq 0$ must vanish. See Secs. 2.3 and 4.3 for more details).

And yet, despite the very restrictive conditions, the stereodynamical portraits at $\theta=0^{\circ}$ are strikingly different from those at $\theta=180^{\circ}$.

The portraits at $\theta=180^{\circ}$ are very similar to those corresponding to a pure $|j=2, \Omega=0\rangle$ state (see Sec. 4.6). Their strong anisotropy is indicative of a direct mechanism favoured by small impact parameter, collinear, head-on collisions. We also note that stereodynamical portraits corresponding to other backward-region scattering angles, $\theta=150-180^{\circ}$, are very similar to the ones presented here.

The situation at $\theta=0^{\circ}$ is rather different; the $\mathrm{D}_{2}$ stereodynamical portraits are 
close to isotropic. Interestingly, the $\mathrm{D}_{2}$ stereodynamical portraits at nearby angles, while also largely isotropic, clearly differ from those obtained at exactly $0^{\circ}$. This is indicative of either a lack of steric constraints, or else a mixture of mechanisms with different stereochemical requirements. Resolution of differential results with regard to product rovibrational states (the subject of the next Section) will show the latter to be the case.

The $\mathrm{D}_{2}$ stereodynamical portraits corresponding to sideways scattering (middle row of Fig. 5.7) are quite similar to others already discussed. Namely, those in the bottom row of Fig. 5.2 (total, integral results at high collision energy), and also those corresponding to $j^{\prime}=9$ in Fig. 5.4 (state-to-state, integral results at high collision energy). The conclusion here is the same reached there, except that here it concerns a shift that is observed with a change of scattering angle rather than collision energy or product state. In going from backward to sideways scattering, the $\mathrm{D}_{2}$ molecular axis, while remaining on the scattering plane, tilts with respect to the reactant-approach direction; reactive collisions change from head-on to side-on; the side-on collisions are largely insensitive to rotational orientation. This effect, which is prominent at high collision energies, can be rationalised by consideration of access to relatively bent collision complex configurations and contributions from large impact parameter collisions, see Secs. 5.2 and 5.3 . As in the case of integral results, the tilt of molecular axis takes place in order to make the linear transition state more accessible.

\subsection{State-to-state differential results}

We now turn to the deepest and most detailed results to be considered in this Chapter: those concerning state-to-state reactions resolved with regard to scattering angle. Here we address the following question: given a certain scattering angle, what reactant polarisation does lead to scattering of products in a particular state into it?

The chief limitation of the results in the previous Sections is that they largely reflect the main characteristics of the dominant, direct reaction mechanism. Exam of state-to-state results with scattering angle resolution allows for a more detailed characterisation of the dominant mechanism, but also for separate consideration of at least one minor mechanism, namely the time-delayed one.

For space and clarity reasons, we will restrict ourselves here to the highest collision energy $(1.506 \mathrm{eV})$ and, unless otherwise stated, to the lowest product vibrational state $\left(v^{\prime}=0\right)$. We would like to point out, however, that the results show little change with $v^{\prime}$, and that although the probabilities of the effects considered here change with collision energy, their nature does not. The conclusions drawn from the data below 


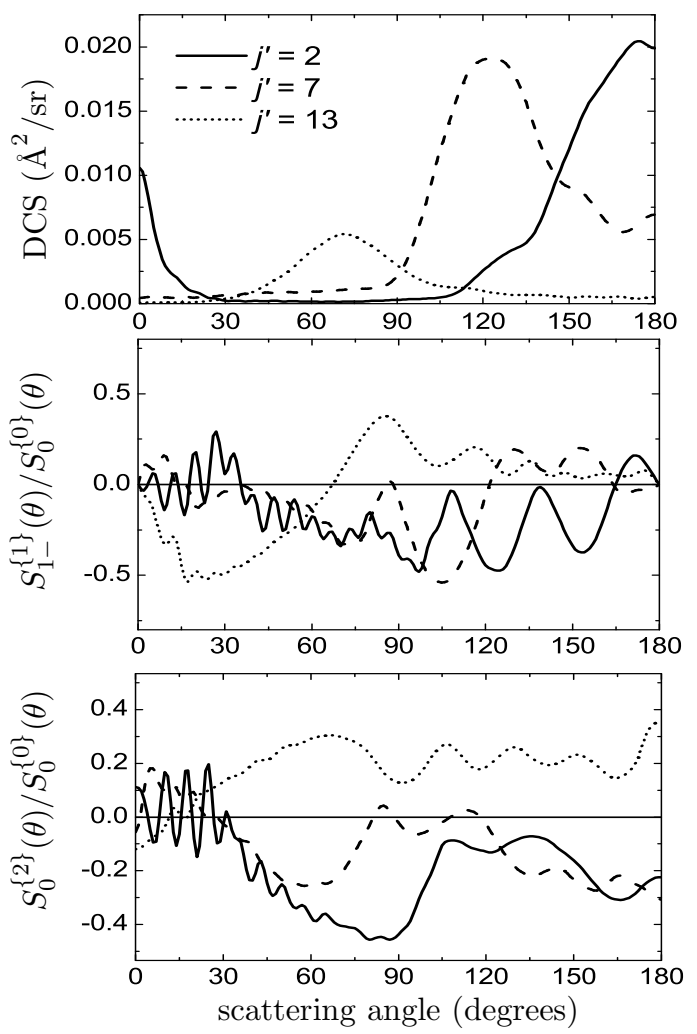

Fig. 5.8.: DCS (top), renormalised PDDCS $\{1,1-\} \quad$ (middle) and renormalised $\operatorname{PDDCS}\{2,0\}$ (bottom) for the $\mathrm{H}+\mathrm{D}_{2}(v=0, j=2) \rightarrow \mathrm{HD}\left(v^{\prime}=0, j^{\prime}\right)+\mathrm{D}$ reaction at $E_{\text {coll }}=1.506 \mathrm{eV}$. The solid, dashed and dotted curves correspond, respectively, to $j^{\prime}=2, j^{\prime}=7$ and $j^{\prime}=13$. In each of the PDDCS panels, the range of the vertical axis matches the allowed range of the PDDCS for $j=2$.

can also be drawn from results at other collision energies or $v^{\prime}$ values for this reaction.

Fig. 5.8 shows the DCS and the renormalised PDDCS $\{1,1-\}$ and PDDCS $\{2,0\}$, for reactions leading to $\mathrm{HD}$ in the $j^{\prime}=2,7$ or 13 rotational state.

Let us first consider the DCSs (top panel of Fig. 5.8). It is clear that most of the reaction probability is associated with reactions leading to backward and/or sideways scattering, and that the higher the $j^{\prime}$ value, the more the DCS is shifted away from strictly backward, $\theta=180^{\circ}$ scattering.

Note, however, that for $j^{\prime}=2$ forward scattering is not negligible: the corresponding DCS features a sharp peak in the $\theta<30^{\circ}$ region.

As for the PDDCSs, their most striking characteristic is something that cannot 
be seen in results summed over product states (e.g., those in Fig. [5.6): they exhibit numerous oscillations. We divide these oscillations in two classes.

The first class comprises the relatively broad oscillations observed for $j^{\prime}=13, j^{\prime}=7$ and backward-scattered $j^{\prime}=2$ products. These oscillations are general (they appear for virtually every product state and collision energy), and are fairly well accounted for by quasiclassical trajectory calculations (see Fig. 4.8 and reference [25]). They reflect attributes of the dominant, direct mechanism, and their rationalisation does not require invocation of quantum effects.

The second class comprises the fast oscillations observed for forward-scattered $j^{\prime}=$ 2 products. These oscillations, which are only found for very low $j^{\prime}$ states, are not accounted for by quasiclassical trajectory calculations (see Fig. 4.8 and reference [25]). They are associated with the minor, "delayed" mechanism, and their rationalisation does require invocation of quantum effects.

In order to provide some rationalisation of the PDDCS of Fig. 5.8, we focus first on the direct mechanism and, in particular, on the $\theta$ values where the dominant peaks of the DCSs have maxima ( $\theta=174,122$ and $72^{\circ}$ for $j^{\prime}=2,7$ and 13 , respectively).

The PDDCS $\{2,0\}$ value is negative for $j^{\prime}=2$ for backward and sideways scattering. As $j^{\prime}$ increases this situation varies: it first become smaller, almost vanishing for intermediate $j^{\prime}$, and becomes positive for $j^{\prime}=13$. In particular, at the angles corresponding to their DCS maxima and nearby angles, the PDDCS $\{2,0\}$ value shifts from negative $\left(j^{\prime}=2\right.$ around $\left.\theta=174^{\circ}\right)$ to nearly vanishing $\left(j^{\prime}=7\right.$ around $\left.\theta=122^{\circ}\right)$ to positive $\left(j^{\prime}=13\right.$ around $\left.\theta=72^{\circ}\right)$. As this value quantifies the $\mathrm{D}_{2}$ alignment with regard to the reactant-approach direction (PDDCS $\{2,0\}<0$ implies preference for $\mathbf{j} \perp \mathbf{k}$ and $\mathbf{r} \| \mathbf{k}$, PDDCS $\{2,0\}>0$ implies preference for $\mathbf{j} \| \mathbf{k}$ and $\mathbf{r} \perp \mathbf{k}$ ), what is seen here can be explained again in terms of the search of a linear transition state. The physical justification for this relationship between reactant alignment and product rotational excitation has been presented above (see discussion of the molecular axis portraits of Fig. 5.4 in Sec. 5.3). As sideways scattering comes from larger impact parameters than backward, the molecule tilts in order to facilitate the adoption of the linear geometry. Consequently, this moment is negative at all scattering angles for low $j^{\prime}$ and positive for the highest $j^{\prime}$.

The PDDCS $\{1,1-\}$ changes in a different way: it undergoes a sign inversion at the $\theta$ value where the DCS has a maximum. Consider, for example, the $j^{\prime}=13$ case, for which the DCS peaks at $\theta=72^{\circ}$. The $\operatorname{PDDCS}\{1,1-\}$ takes positive values at $\theta \gtrsim 72^{\circ}$, and negative values at $\theta \lesssim 72^{\circ}$. The same happens for $j^{\prime}=7$ around $\theta \approx 122^{\circ}$, and for $j^{\prime}=2$ around $\theta \approx 174^{\circ}$.

In order to rationalise the effects described in the previous paragraph, we take 

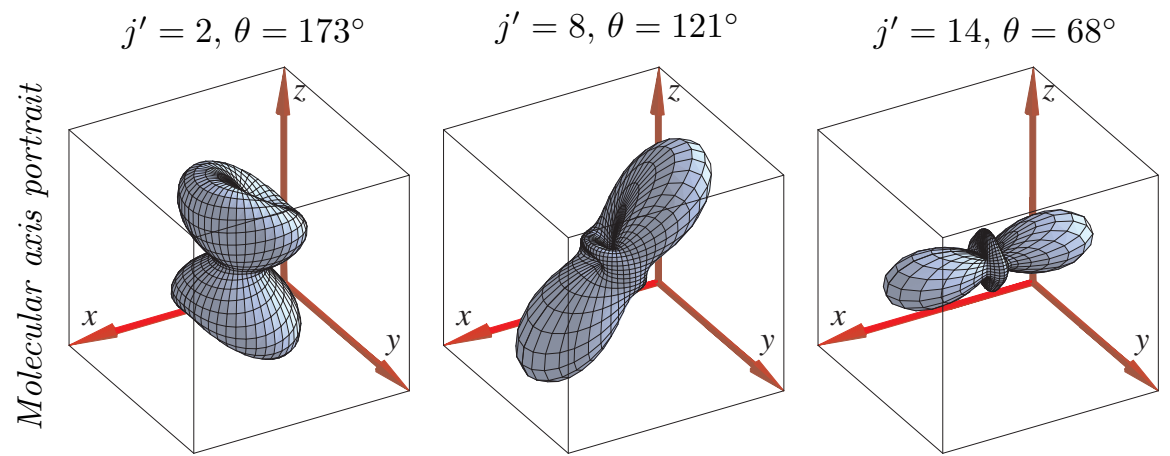

Fig. 5.9.: $\mathrm{D}_{2}(v=0, j=2)$ molecular axis portraits prepared using renormalised PDDCSs for $E_{\text {coll }}=1.506 \mathrm{eV}$ reactions into $v^{\prime}=0$ and $j^{\prime}=2,7$ or 13 . The scattering angles considered here are those associated with maxima of the DCSs on the top panel of Fig. 5.8 .

into account the fact that the $\operatorname{PDDCS}\{1,1-\}$ quantifies rotational orientation with regard to the $y \equiv \mathbf{k} \times \mathbf{k}^{\prime}$ axis $(\operatorname{PDDCS}\{1,1-\}>0$ implies preference for $\mathbf{j}$ along $+y$, $\operatorname{PDDCS}\{1,1-\}<0$ implies preference for $\mathbf{j}$ along $-y)$. We also take into account the cartoon on Fig. 5.5. Using $j^{\prime}=13$ as an example, the rationalisation is as follows. The DCS maximum appears at $\theta=72^{\circ}$, the $\operatorname{PDDCS}\{1,1-\}=0$. At $\theta \gtrsim 72^{\circ}$ the reaction probability shifts towards lower $j^{\prime}$ values, and as a consequence formation of $\operatorname{HD}\left(j^{\prime}=\right.$ 13) requires positive $\mathbf{j}$ orientation (that is, $\operatorname{PDDCS}\{1,1-\}>0$ ). At $\theta \lesssim 72^{\circ}$ the reaction probability shifts towards higher $j^{\prime}$ values, and as a consequence formation of $\operatorname{HD}\left(j^{\prime}=13\right)$ correlates with negative $\mathbf{j}$ orientation (that is, $\left.\operatorname{PDDCS}\{1,1-\}<0\right)$.

As always, the information content of the polarisation moments (all of them, not only the ones on Fig. 5.8) is best conveyed by stereodynamical portraits. Some of these, which have been selected so that details of the direct mechanism are depicted as clearly as possible, are shown on Figs. 5.9 and 5.10 . As above, the results presented are for reactions into $v^{\prime}=0$ at $E_{\text {coll }}=1.506 \mathrm{eV}$.

Fig. 5.9 presents $\mathrm{D}_{2}(v=0, j=2)$ molecular axis portraits associated with different combinations of product rotational state and scattering angle; the combinations of $\theta$ and $j^{\prime}$ values are those associated with maxima of the DCSs on the top panel of Fig. 5.8. What this figure most clearly illustrates is the correlation between the tilt of the $\mathrm{D}_{2}$ axis and the product rotational state and scattering angle. Larger tilts, associated as already discussed with larger impact parameters and more HDD bending around its linear equilibrium geometry, correlate with higher product rotational excitation and with smaller scattering angles. 


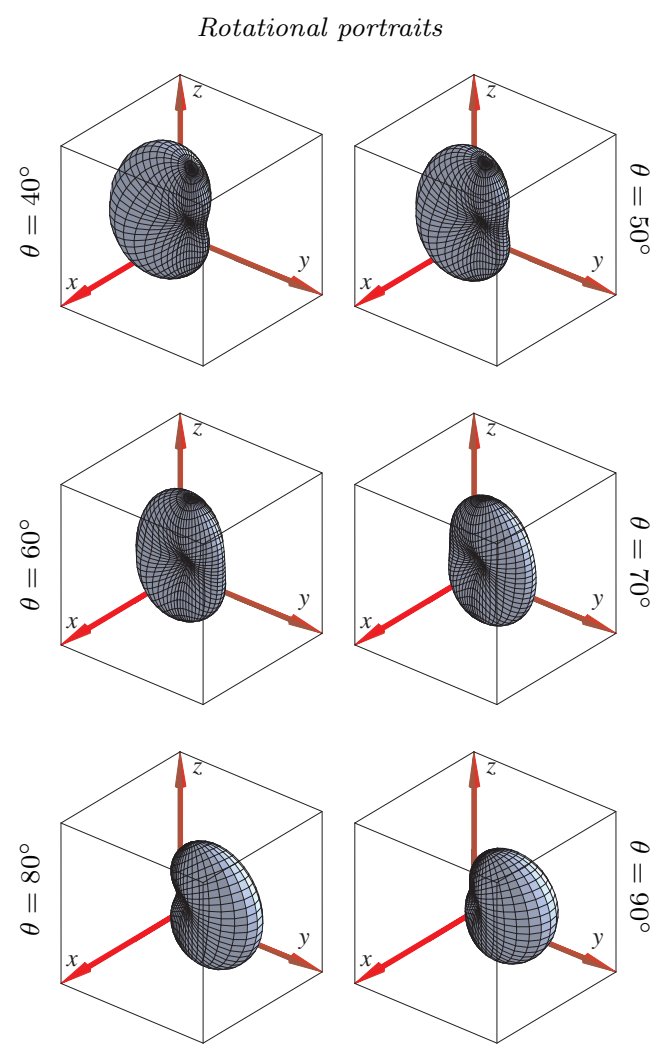

Fig. 5.10.: $\mathrm{D}_{2}(v=0, j=2)$ rotational portraits prepared using renormalised PDDCSs for $E_{\text {coll }}=1.506 \mathrm{eV}$ reactions leading to $\operatorname{HD}\left(v^{\prime}=0, j^{\prime}=13\right)$. The $\theta$ values used span the scattering angle region where the reaction DCS (dotted line on the top panel of Fig. (5.8) has its maximum.

As for Fig. 5.10, it shows $\mathrm{D}_{2}(v=0, j=2)$ rotational portraits meant to illustrate the correlation between $\mathrm{D}_{2}$ rotational polarisation and scattering angle. The portraits included are for reactions into $\operatorname{HD}\left(v^{\prime}=0, j^{\prime}=13\right)$ at scattering angles in the region where the DCS of this reaction has its maximum, see top panel of Fig. 5.8. In this case, it turns out that the most important effect (inversion of the $\mathrm{D}_{2}$ rotational orientation upon passage through the scattering angle where the DCS hits its maximum) is clearly described by one polarisation moment only, the one we have separately discussed: $\operatorname{PDDCS}\{1,1-\}$. The other noticeable effect (preference for $\mathbf{j}$ to lie in the $y z$ plane) results from the combined effect of all PDDCSs, and is represented in a more telling way by the preference for the $\mathrm{D}_{2}$ interatomic axis to lie near the $x$ direction, see right panel of Fig. 5.9. 


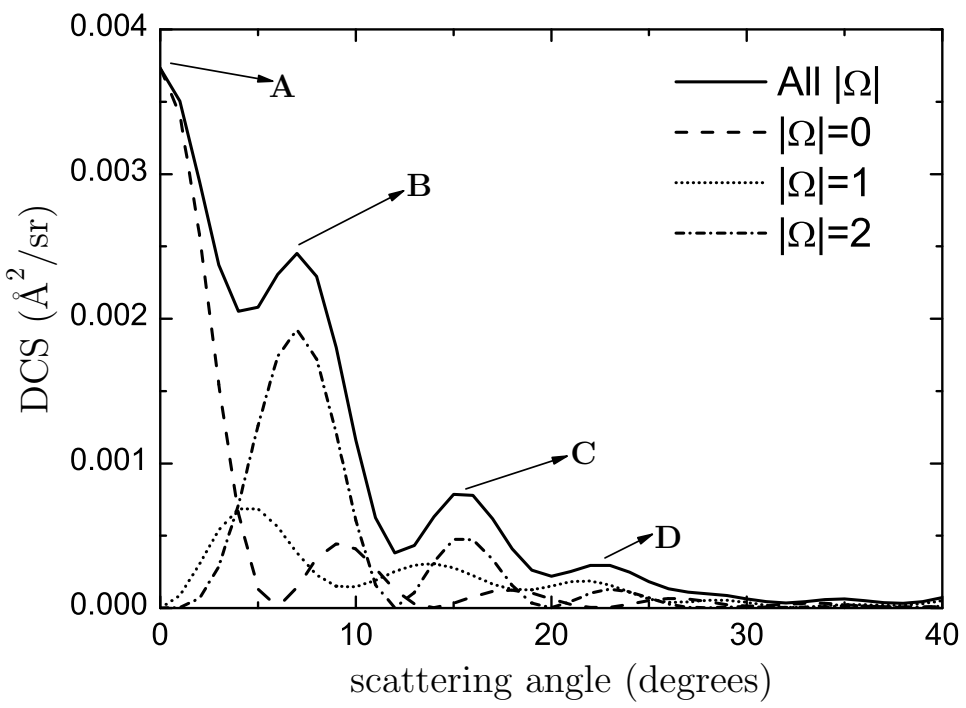

Fig. 5.11.: Differential cross section, in the forward scattering region, of the $\mathrm{H}+\mathrm{D}_{2}(v=$ $0, j=2) \rightarrow \operatorname{HD}\left(v^{\prime}=1, j^{\prime}=0\right)+\mathrm{D}$ reaction at $E_{\text {coll }}=1.506 \mathrm{eV}$. The solid line represents the full result; the other lines represent partial contributions from selected $\mathrm{D}_{2}$ helicities.

Having discussed the dominant, direct reaction mechanism, we now turn to the delayed mechanism responsible for forward scattering of products in low rotational states. As illustrated by the polarisation moments associated with $j^{\prime}=2$ in Fig. 5.8, delayed collisions are characterised by PDDCSs that oscillate very fast with scattering angle. These oscillations are found for all PDDCSs, not only those included in Fig. 5.8 .

Fig. 5.11 shows a close-up of the oscillations of the DCS in the forward scattering region. The results in the figure are for reaction into $\operatorname{HD}\left(v^{\prime}=1, j^{\prime}=0\right)$ at $E_{\text {coll }}=1.506 \mathrm{eV}$, and include not only the full DCS, but also partial contributions from selected absolute values of the $D_{2}$ helicity, namely $|\Omega|=0,|\Omega|=1$ and $|\Omega|=2$.

It is clear that the partial DCSs oscillate more markedly than the full DCS, and that the oscillations that survive in the full results can be attributed to particular $|\Omega|$ values. The peak labelled as $\mathrm{A}$ in Fig. 5.11 is associated with $|\Omega|=0$, while peaks $\mathrm{B}, \mathrm{C}$ and $\mathrm{D}$ are mostly due to $|\Omega|=2$ (in fact, summation of the contributions from $|\Omega|=0$ and $|\Omega|=1$ results in a partial DCS that, apart from the sharp peak at $\theta=0$, shows no prominent maxima or minima in the $\theta<40^{\circ}$ region; this "oscillation-free" partial DCS is not shown on the figure).

It already remains the problem of determining the origin of the oscillations for each 
$|\Omega|$ resolved DCS. A partial wave analysis ${ }^{4}$ of the forward scattering oscillations shows that the various DCS peaks are due to various groups of high- $J$ partial waves (note, however, that no peak can be attributed to a particular total angular momentum; angular features such as these come from interference between different partial waves).

Although the discussion in the last two paragraphs is based on consideration of the DCS, the conclusion is also valid for PDDCSs. Their oscillations are due to interference between (that is, coherent summation of) particular groups of large- $J$ partial waves. In the case of PDDCSs with $q=0$ (the only case when the PDDCSs are separable into different $|\Omega|$ contributions), the oscillations can furthermore be attributed to incoherent contributions from separate $|\Omega|$ values.

From the point of view of the mechanism, these interferences between different $J$ values and the corresponding moments oscillations can be explained in terms of the nearside-farside (NF) analysis of the DCS developed by Connor and co-workers [115, 116]. They related the forward oscillations of the DCS at small values of $j^{\prime}$ to the interferences between NF reactive collisions. Taking into account the previous discussion, it would be reasonable to attribute the PDDCSs oscillations to the same effect, that is, to the NF interferences. This fact, that has been demonstrated in several cases [115, 116, 119], also appears in QCT calculations. It is possible to show that for forward scattering there are two kind of collisions: those with a positive deflection angle (near $0^{\circ}$ ) corresponding to nearside collisions, and those with a negative deflection angle, and thus farside collisions. Of course, in classical mechanics there are not interferences between trajectories ending in the same scattering angle (absolute value of the deflection angle) and the oscillations of the PDDCS in the forward region do not show up.

In any case, the PDDCS oscillations dramatically affect the $\mathrm{D}_{2}$ rotational portraits, which illustrate very eloquently the extent to which the stereodynamics of the delayed reaction can change within a very narrow scattering angle range. Proof of this is given by Fig. 5.12, which shows molecular axis and rotational $\mathrm{D}_{2}$ stereodynamical portraits for reaction into $\operatorname{HD}\left(v^{\prime}=1, j^{\prime}=0\right)$ at $E_{\text {coll }}=1.506 \mathrm{eV}$. Although the scattering angles considered in the figure $\left(\theta=5,10\right.$ and $\left.13^{\circ}\right)$ are very close to each other, the intrinsic $\mathbf{r}$ and $\mathbf{j}$ distributions contrast strongly. What is more, they can take rather odd shapes (consider, for example, the results at $\theta=5^{\circ}$ ).

Comparison between the stereodynamical portraits shown on Fig. 5.12 and those presented earlier shows that the unusual chemistry of the delayed mechanism cannot be perceived in its full variety unless the reaction properties are considered at a stateto-state level and with scattering angle resolution. Summation over only a few product

\footnotetext{
${ }^{4}$ An analysis consisting of to consider separately the reactivity due to different intervals of $J$ values
} 


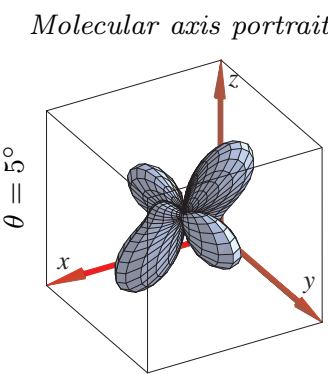

Rotational portrait
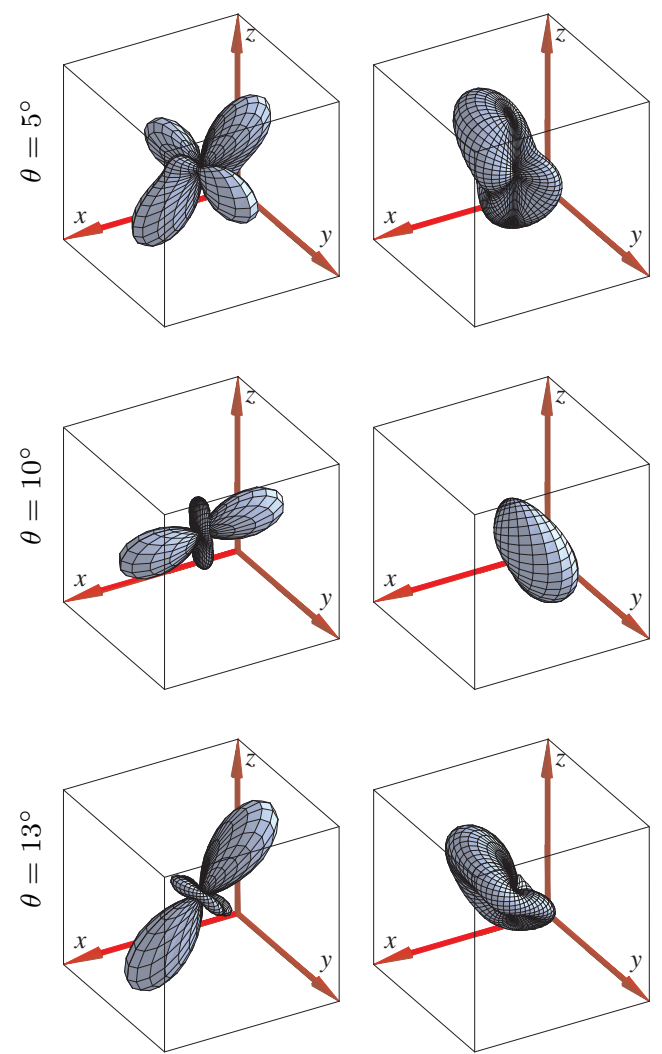

Fig. 5.12.: Molecular axis and rotational $\mathrm{D}_{2}(v=0, j=2)$ stereodynamical portraits prepared using renormalised state-to-state PDDCSs for forward-scattering reactions into $\mathrm{HD}\left(v^{\prime}=1, j^{\prime}=0\right)$ at $E_{\text {coll }}=1.506 \mathrm{eV}$.

states, or else integration over a small scattering angle range in the close vicinity of $\theta=0^{\circ}$, results in fairly isotropic stereodynamical portraits. 


\section{Control and mechanism of the $\mathbf{F}+\mathbf{H}_{2}$ reactive collisions}

If chemical reactions were classified according to the attention and effort historically devoted to each of them, the $\mathrm{F}+\mathrm{H}_{2}$ reaction and its isotopical variants would occupy the second position, just behind the $\mathrm{H}_{3}$ system. This statement suggests that the study of the $\mathrm{F}+\mathrm{H}_{2}$ process is the natural continuation of the work presented so far and, in fact, it will constitute the goal of this Chapter.

After presenting a brief overview of the reaction features that will be significant for our study (Sec.6.1) and of the quantum mechanical calculations performed (Sec.6.2), the effect of $\mathbf{j}$ (the $\mathrm{H}_{2}$ rotational angular momentum) polarisation on the reaction

$$
\mathrm{F}+\mathrm{H}_{2}(v=0, j=2) \rightarrow \mathrm{HF}+\mathrm{H}
$$

outcome at $E_{\text {coll }}=0.079,0.119$ and $0.148 \mathrm{eV}$ (the energies correspond to the crossed beams experiments performed by Lee and co-workers [120]) will be thoroughly analysed (Secs. 6.3, 6.4, 6.5 and 6.6) in order (i) to estimate the extension of the control that can be obtained and (ii) to shed light on the mechanism of the collisions.

The rationalisation of the results in terms of the mechanism will share some characteristics with the analysis performed for the $\mathrm{H}+\mathrm{D}_{2}$ collisions:

- Two different mechanisms have to be invoked in order to explain the results. A detailed consideration of their features will show that they are nothing but the counterparts of the direct and delayed mechanisms introduced in connection to the $\mathrm{H}+\mathrm{D}_{2}$ reactive collisions. We will continue, therefore, referring to them with the same names.

- The direct mechanism is responsible for products scattered in the backwardsideways region, mainly through low to medium impact parameter collisions. In practise, this includes most of the reaction probability.

- The delayed mechanism is related to high impact parameter collisions leading to forward scattering (concentrated on $v^{\prime}=2$ and 3 and low-medium $j^{\prime}$ values). 
The existence of this mechanism only becomes evident when angle resolved state-to-state quantities are considered and, because of this, its analysis will not be tackled till Sec. 6.6.

These similarities between the $\mathrm{F}+\mathrm{H}_{2}$ and the $\mathrm{H}+\mathrm{D}_{2}$ reactions do not involve, however, that neither the direct nor the delayed mechanisms are exactly identical for both reactions.

As in the former Chapter, the presentation of the results will proceed from general to detailed quantities. It is now convenient, however, to consider separately the reactive collisions leading to each vibrational level of the HF product molecule. Therefore, no total (averaged over all final states) but vibrationally resolved (averaged only over rotational levels) quantities will be considered.

\subsection{Reaction general features}

The $\mathrm{F}+\mathrm{H}_{2}$ reaction is the prototypical exothermic exchange reaction. Its study has been the subject of a large number of experimental and theoretical works (see [77, 121] and references therein) that furnish with a thorough description of the reaction.

Among the experimental works, those due to Neumark and co-workers (they studied the $\mathrm{FH}_{2}^{-}$photoelectron spectra $\left.122,123,124\right)$ and Lee and co-workers (a $\mathrm{F}+\mathrm{H}_{2}$ crossed beams experiment [120]) have to be pointed out. The former provided with a description of the $\mathrm{F}+\mathrm{H}_{2}$ reaction in the transition state (TS) region and the second proved two interesting characteristics of the same process:

- The most populated vibrational levels of the HF product molecules are $v^{\prime}=2$ and 3. In other words, the products vibrational population is inverted.

- The $\operatorname{HF}\left(v^{\prime}=0,1,2\right)$ molecules are predominantly formed in the backwardsideways directions. On the contrary, the DCS for $\operatorname{HF}\left(v^{\prime}=3\right)$ displays a noticeable forward peak that sticks up out of the backward-sideways DCS.

Several fruitless attempts to theoretically reproduce the experimental results culminated with the development of the Stark and Werner PES (SW) [125]. Calculations ran on the surface were able to reproduce (i) the $\mathrm{FH}_{2}^{-}$photoelectron spectra [126] and (ii) most of the features of the crossed beams experiments (including the two features formerly indicated ${ }^{1}$ ) [127, 128, 129, 130]. These good results have determined that,

\footnotetext{
${ }^{1}$ Not all the reaction observables were reproduced with the same exactitude. In particular, the amount of the forward scattering in $v^{\prime}=3$ is not properly reproduced neither by QCT nor quantum mechanical calculations. While the former underestimate the magnitude of the peak, the second overestimate it 121
} 


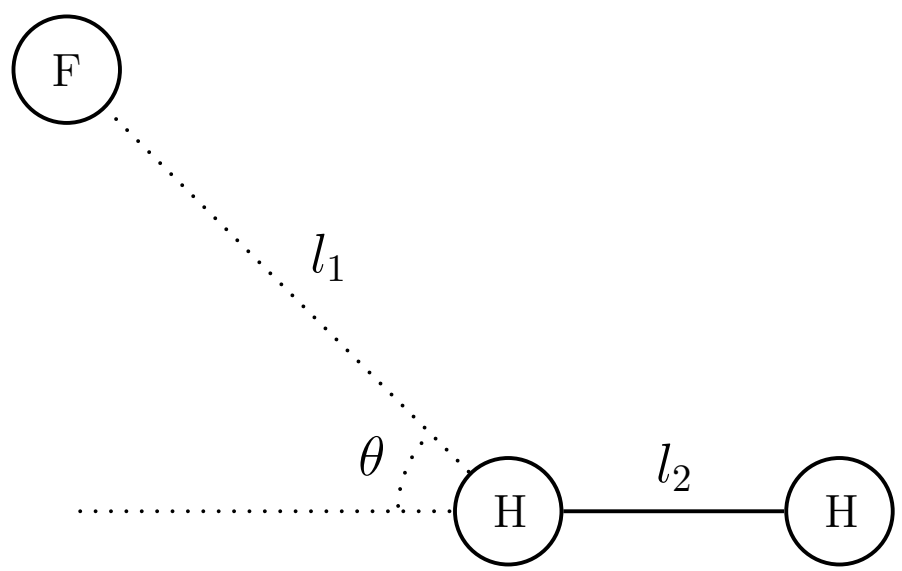

Fig. 6.1.: Transition state for the SW PES $\left(l_{1}=1.55 \AA, l_{2}=0.77 \AA\right.$ and $\left.\theta=61^{\circ}\right)$.

until now, the SW PES is the benchmark for the theoretical study of the reaction and, as such, it will be employed for our calculations.

The transition state of the SW PES [125] is bent (its geometry is presented in Fig. 6.1), "early" (located at the end of the reactants valley) and the bending potential is fairly flat. The surface possesses two Van-der-Walls wells, one on the reactants valley (T-shaped) and the other on the products valley (linear). The barrier height is $0.066 \mathrm{eV}(1.53 \mathrm{kcal} / \mathrm{mol})$ for the TS and $0.083 \mathrm{eV}(1.92 \mathrm{kcal} / \mathrm{mol})$ for the collinear barrier (the insertion is energetically disfavored as the corresponding barrier is remarkably higher).

The existence of reactive resonances for the $\mathrm{F}+\mathrm{H}_{2}$ reaction had been predicted during the 1970s [131] and no effort has been spared ever since in order to search evidences supporting those predictions. The forward $\operatorname{HF}\left(v^{\prime}=3\right)$ peak found in Lee and co-workers experiments [120] was initially attributed to one of such resonances, although later QCT [127, 128] and QM [129] calculations over the SW PES did not support this statement, indicating that the $\mathrm{HF}\left(v^{\prime}=3\right)$ forward scattering is probably not due to the existence of a resonance. However, Skodje and co-workers unequivocally characterised the effect of a reactive resonance [132] on the $\mathrm{F}+\mathrm{HD}(v=0, j=0)$ isotopic variant of the reaction at collision energies well below those employed in Lee's experiments (the resonance appears at $0.021 \mathrm{eV}(0.5 \mathrm{kcal} / \mathrm{mol}))$ and suggested 133 . that the same behaviour should be found for the $\mathrm{F}+\mathrm{H}_{2}$ variant in the same range of energies. Two very recent works $[134,135]$ have confirmed $^{2}$ this suggestion by proving

\footnotetext{
${ }^{2}$ Using a modified version of the SW PES
} 
that the $v^{\prime}=2$ forward scattering ${ }^{3}$ is related to the presence of reactive resonances (the resonances correspond to quasi-bound states over the vibrationally adiabatic potential correlating with $v^{\prime}=3$ which, however, decay into the $v^{\prime}=2$ channel).

This is not the fist study directly on indirectly focused on the $\mathrm{F}+\mathrm{H}_{2}$ reaction stereodynamics. Previous works furnish with some hints about the process mechanism:

- The influence of the T-shaped Van-der-Walls well on the reaction depends on the reactants rotational excitation [128]: while it has an orientational effect on the $\mathrm{F}+\mathrm{H}_{2}(v=0, j=0)$ reaction, its role becomes less important as the reactants get rotationally excited (it diminishes for $j=1$ and is expected to disappear for larger $j$ values).

- Reactive collisions leading to vibrationally excited products (specially $\mathrm{HF}\left(v^{\prime}=\right.$ 3)) tend to be coplanar [136. As $v^{\prime}$ decreases this tendency becomes less marked.

- The TS influence manifests in a preference (a higher reactivity) for those collision geometries that resembles the TS geometry [72].

that will be useful to interpret our results.

\subsection{Reactive scattering calculations}

The quantum mechanical intrinsic moments have been the starting point for our study. These moments were worked out from the scattering matrices in the helicity representation obtained through time independent quantum mechanical calculations carried out with the ABC code [75] on the Stark and Werner PES [125] at the collision energies considered in this Chapter: 0.079, 0.119 and $0.148 \mathrm{eV}$.

The ABC code employs a coupled channel hyperspherical coordinate method to solve the nuclear Schrödinger equation on a single potential energy surface. Prior to perform the calculations, the values of certain execution parameters have to be set in such a way that results convergence is ensured. In order to set the suitable values of these parameters, the total reaction probabilities and product rotational populations obtained with different parameters sets and for zero total angular momentum reaction were compared, concluding that: the optimum cutoff energy for the rovibrational basis set is $1.75 \mathrm{eV}$, the suitable maximum rotational angular momentum and helicity quantum numbers are 21 and 7 respectively and the integration has to be performed till a maximum hyperradius value of $15 a_{\circ}$ ( $a_{\circ}$ represents the Borh radius). The number of partial waves necessary to obtain convergence was 25 .

\footnotetext{
${ }^{3}$ Owing to experimental limitations, the forward scattering for $v^{\prime}=2$ was not detected by Lee's experiments
} 


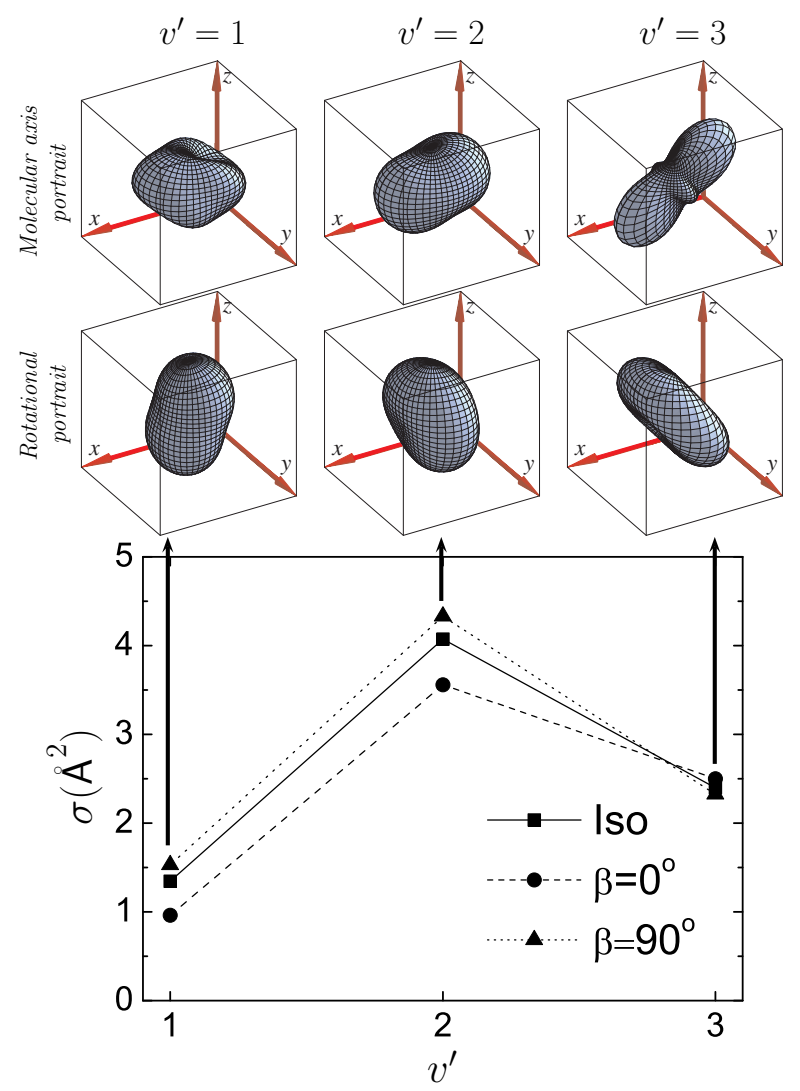

Fig. 6.2.: Integral cross section of the $\mathrm{F}+\mathrm{H}_{2}(v=0, j=2) \rightarrow \mathrm{HF}\left(v^{\prime}, j^{\prime}=\right.$ all $)+\mathrm{H}$ reaction at $E_{\text {coll }}=0.148 \mathrm{eV}$ as a function of $v^{\prime}$ for different $\mathrm{H}_{2}$ alignment directions, and molecular axis and rotational $\mathrm{H}_{2}$ stereodynamical portraits prepared using vibrationally resolved PPs for the same reaction.

\subsection{Vibrationally resolved integral results}

The aim of this Section is to study the mechanism and control of reactive collisions (6.1) leading to HF formation into a given vibrational state.

A detailed analysis of the reaction stereodynamics for the three considered energies $(0.079,0.119$ and $0.148 \mathrm{eV})$ did not show significant differences between the results corresponding to each of them. In order to present the results in the clearest way, we will concentrate on the analysis of the largest one because it possesses the largest number of open products channels and, therefore, it allows for the optimum characterisation of those mechanism features depending on the products internal state. However, it should be borne in mind that the conclusions hold for the three energies. 
The Section goal can be achieved by means of the information contained in Fig. 6.2 the vibrationally resolved $\left(v^{\prime}=1,2\right.$ and 3 ) integral cross section for unpolarised (Iso) and polarised ${ }^{4}\left(\beta=0\right.$ and $\left.90^{\circ}\right)$ reactants and the corresponding intrinsic portraits showing $\mathbf{j}$ and $\mathbf{r}$ ( $\mathrm{H}_{2}$ internuclear axis) distributions when the reaction happens.

Exam of the bottom panel of Fig. 6.2 shows that the vibrationally resolved integral cross section is relatively insensitive to the reactants polarisation:

- For $v^{\prime}=1$ and 2, head-on $\left(\beta=0^{\circ}\right)$ collisions reduce the cross section and side-on $\left(\beta=90^{\circ}\right)$ collisions slightly increase it.

- For $v^{\prime}=3$ the cross section is almost independent of the reactants preparation.

As it will be proved in next Section, this lack of control for the overall reaction does not correspond to absence of steric requirements but it is the consequence of a cancellation due to the the summation over $j^{\prime}$.

More information is provided by the molecular axis (top panel of Fig. 6.2) and rotational (middle panel of Fig. 6.2) portraits. The former show that collisions leading to $\operatorname{HF}\left(v^{\prime}=3\right)$ formation happen with $\mathbf{r}$ preferably placed on the scattering plane and tilted approximately $45^{\circ}$ with respect to the $z \equiv \mathbf{k}$ axis (this tilting is such that the "first" H atom ${ }^{5}$ lies towards the $x>0$ half of the scattering $x z$ plane). As the products vibrational excitation decreases, the portraits become progressively more isotropic, their tilting increases and the $\mathbf{r}$ propensity for being placed on the scattering plane vanishes. These findings are consistent with reference [136] and confirm that, at least for reactions leading to vibrationally excited products, the collisions are essentially coplanar.

Regarding the rotational portraits, their shape display two remarkable changes as $v^{\prime}$ increases: (i) the portraits tilt with respect to the $z$ axis and (ii) show an increasing (decreasing) preference for being aligned along the $y(x)$ axis. While the former change is due to the $\operatorname{PP}\{2,0\}$ moment passage from slightly positive $\left(v^{\prime}=1\right)$ to almost zero $\left(v^{\prime}=3\right)$ values, the second is related to $\operatorname{PP}\{2,2+\}$, whose values change from almost zerc $^{6}$ for $v^{\prime}=1$ to fairly negative ${ }^{7}$ for $v^{\prime}=3$.

\footnotetext{
${ }^{4}$ Results for the preparation corresponding to the magic angle $\left(\beta=54.74^{\circ}\right)$ are not displayed as this preparation leads to cross sections that are almost indistinguishable from their isotropic counterparts

${ }^{5}$ The one closest to the $\mathrm{F}$ atom approaching along the $\mathbf{k}$ direction

${ }^{6}$ Regarding alignment perpendicular to $\mathbf{k}$, $\mathbf{j}$ does not differentiate between the $x$ and $y$ directions

${ }^{7} \mathbf{j}$ preferably aligned along the $y$ axis instead of along $x$
} 


\subsection{State-to-state integral results}

The mechanism and control of $\mathrm{F}+\mathrm{H}_{2}(v=0, j=2)$ reactive collisions leading to formation of $\mathrm{HF}$ molecules in well defined rovibrational states is now considered.

Figs. 6.3 and 6.4 will be the starting point for the discussion. They illustrate the effect of $\mathrm{H}_{2}$ rotational angular momentum (j) polarisation on the

$$
\mathrm{F}+\mathrm{H}_{2}(v=0, j=2) \rightarrow \mathrm{HF}\left(v^{\prime}=2-3, j^{\prime}\right)+\mathrm{H}
$$

reactive state-to-state integral cross sections and provide with the intrinsic portraits for selected values of $j^{\prime}$. Results for reactive collisions whose product is $\operatorname{HF}\left(v^{\prime}=1\right)$ are not presented because they do not significatively differ from those for $v^{\prime}=2$ (the main divergences will be related to the diminishing coplanar character of the collisions as $v^{\prime}$ decreases. This fact determines that the $v^{\prime}=1$ state-to-state internuclear axis portraits are less concentrated on the scattering plane than their $v^{\prime}=2$ counterparts).

Collisions leading to $v^{\prime}=2$ (Fig. 6.3) and $v^{\prime}=3$ (Fig. 6.4) are distinctly affected by the reactants polarisation

- Formation of low rotationally excited $\operatorname{HF}\left(v^{\prime}=2\right)$ molecules is favoured by sideon $\left(\beta=90^{\circ}\right)$ collisions and disfavoured by head-on collisions $\left(\beta=0^{\circ}\right)$ (see Fig. 6.3). This behaviour gets inverted when the HF molecules are rotationally excited so that the cross section decreases (increases) for side-on (head-on) collisions. Reactants preparation corresponding to $\beta=54.74^{\circ}$ (magic angle) leave the cross section almost unchanged.

- When the reaction outcome consist of $\operatorname{HF}\left(v^{\prime}=3\right)$ molecules (see Fig. 6.4), the situation turns out to be slightly more complicated. In general, the cross section is less sensitive to reactants alignment than when $\operatorname{HF}\left(v^{\prime}=2\right)$ is formed. The most significant changes are related to head-on collisions, which augment the cross section for low and high $j^{\prime}$ values and reduce it for medium $j^{\prime}$ values. Sideon collisions effect is approximately the contrary (although its effectiveness on changing the reaction probability is lower): the cross section decreases when the products rotational excitation is low and increases when it has medium values. As for the magic angle preparation, it does not significantly modify the reaction probability (only for medium $j^{\prime}$ values the changes are noticeable).

As in the former Section, this information is complemented by the analysis of the internuclear axis and rotational portraits. Regarding the former (top row on Figs. 6.3 and 6.4 ), two features are important: their tilting with respect to the $z$ axis (k) and 

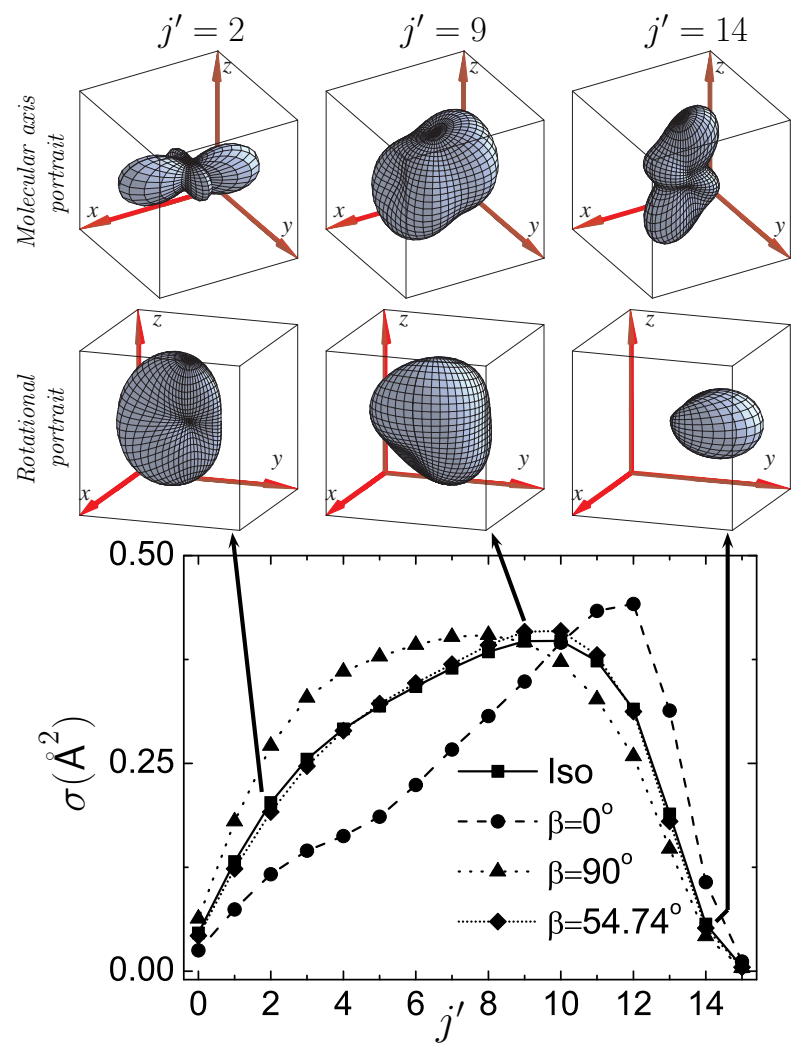

Fig. 6.3.: Integral cross section of the $\mathrm{F}+\mathrm{H}_{2}(v=0, j=2) \rightarrow \mathrm{HF}\left(v^{\prime}=2, j^{\prime}\right)+\mathrm{H}$ reaction at $E_{\text {coll }}=0.148 \mathrm{eV}$ as a function of $j^{\prime}$ for different $\mathrm{H}_{2}$ alignment directions, and molecular axis and rotational $\mathrm{H}_{2}$ stereodynamical portraits prepared using state-to-state PPs for the same reaction.

their propensity for being placed (or not) on the scattering plane. Both characteristics largely change with $v^{\prime}$ and $j^{\prime}$ :

- for $v^{\prime}=2$ collisions, the internuclear axis ( $\left.\mathbf{r}\right)$ tend to be perpendicular (parallel) to $\mathbf{k}$ when the products rotational excitation is low (high). Large and small values of $j^{\prime}$ correlate with collisions where $\mathbf{r}$ prefers to be located on the scattering plane while, on the contrary, collisions leading to medium $j^{\prime}$ values do not display such preference.

- for $v^{\prime}=3$ collisions, $\mathbf{r}$ tend to be $45^{\circ}$ tilted with respect to $\mathbf{k}$ when the products rotational excitation is low and perpendicular when the excitation is high. The internuclear axis portraits is highly concentrated on the scattering plane 


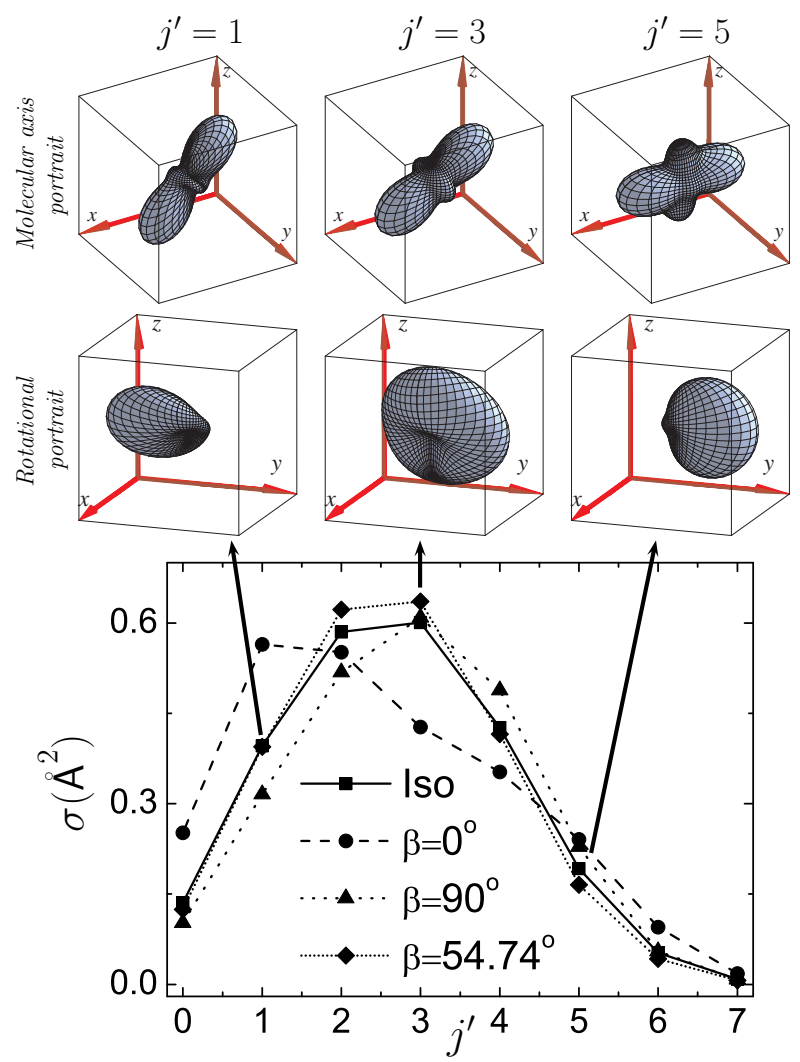

Fig. 6.4.: Integral cross section of the $\mathrm{F}+\mathrm{H}_{2}(v=0, j=2) \rightarrow \mathrm{HF}\left(v^{\prime}=3, j^{\prime}\right)+\mathrm{H}$ reaction at $E_{\text {coll }}=0.148 \mathrm{eV}$ as a function of $j^{\prime}$ for different $\mathrm{H}_{2}$ alignment directions, and molecular axis and rotational $\mathrm{H}_{2}$ stereodynamical portraits prepared using state-to-state PPs for the same reaction.

regardless $j^{\prime}$.

This results can be rationalised by considering that the reaction happens to a large extent through direct collisions ${ }^{8}$ where no formation of any short-lived complex happens and whose features are highly determined by the necessity of making easier the access to the TS region. This last statement, supported by (i) the former Chapter analysis, (ii) the early character of the TS for the $\mathrm{F}+\mathrm{H}_{2}$ reaction and (iii) the contains of reference [72, will be the basis of a simple model that accounts very well for the tilting changes displayed by the state-to-state portraits.

The model is based on the three cartoons included in Fig. 6.5. By assuming that

${ }^{8}$ We will see later (Sec.6.6) that a second mechanism is necessary to fully account for all the results 

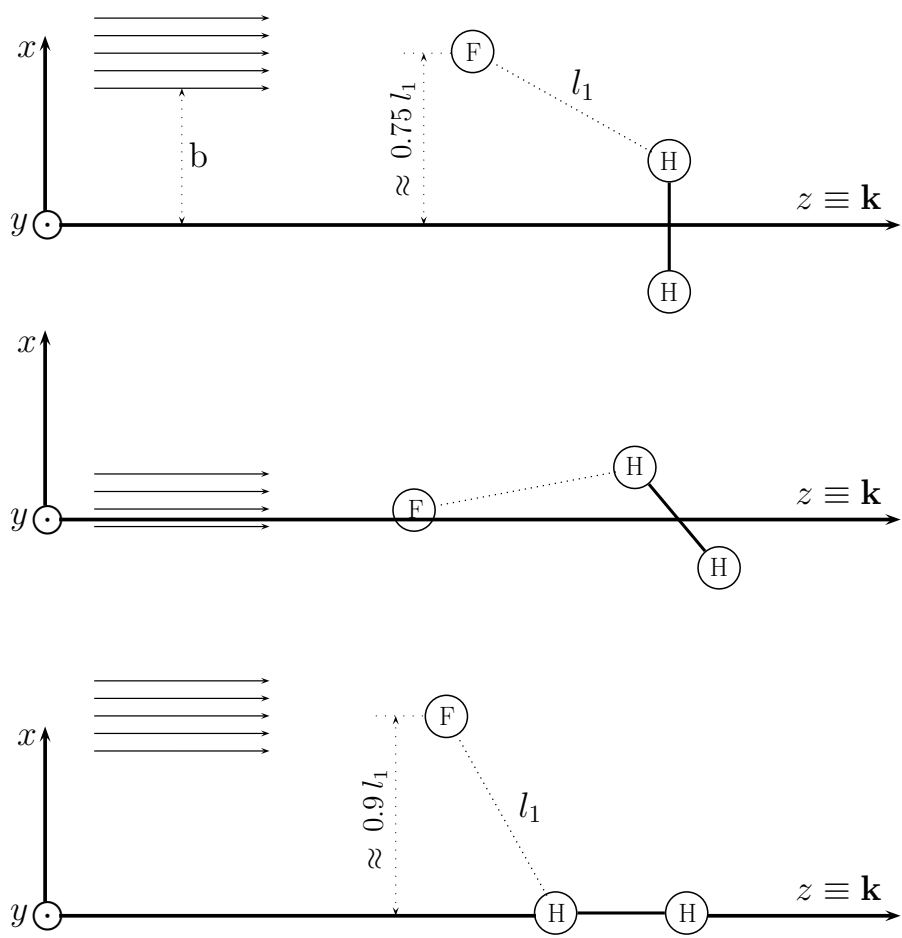

Fig. 6.5.: Cartoons showing the range of impact parameters (b) which, for coplanar reactions (where the paper surface represents the scattering plane $(x z)$ ), would be expected to favour the access to TS geometry when the $\mathrm{H}_{2}$ molecule is initially perpendicular (top), $45^{\circ}$ tilted (medium) and parallel (bottom) to the incoming direction $\mathbf{k}$. The "optimum" impact parameters for side-on $\left(\approx 0.75 l_{1}\right)$ and head-on $\left(\approx 0.9 l_{1}\right)$ collisions have been estimated from the TS geometry (Fig. 6.1) in terms of $l_{1}(1.55 \AA$, the shortest F-H distance in the TS).

reactive collisions are near-side ${ }^{9}$, they show the impact parameter range that facilitates ${ }^{10}$ the system access to the TS geometry for coplanar collisions where the $\mathrm{H}_{2}$ molecule is initially perpendicular (top panel), $45^{\circ}$ tilted (medium panel) and parallel (bottom panel) to the reactants approach direction $\mathbf{k}$. Working on the hypothesis that the direct mechanism highly reflects the steric requirements for the system to acquire the TS geometry, Fig. 6.5 shows the expected correlation between impact parameter and collision geometry for reactions dominated by that mechanism:

\footnotetext{
${ }^{9}$ Justification of this statement will be provided in Sec. 6.6

${ }^{10}$ The model completely neglects any orientational effect due to the PES (in particular, those due to the reactants Van-der-Walls well). As it was pointed out in Sec. 6.1 this assumption is expected to be valid for rotationally excited reactants like $\mathrm{H}_{2}(v=0, j=2)$
} 
- small impact parameters correlate with collisions where the internuclear axis is tilted with respect to $\mathbf{k}$,

- medium impact parameters with side-on collisions and

- large impact parameters with head-on collisions. The centrifugal barrier becomes important for these collisions, determining that they will be prone to look for the minimum energy to cross the TS.

These relations can be easily extended to reactions that are not fully coplanar just by considering how the the range of suitable impact parameters for each collision geometry in Fig. 6.5 is modified by (i) moderate rotations of the F-H-H complex around the $\mathrm{H}-\mathrm{H}$ axis (getting the $\mathrm{F}$ atom out of the scattering plane, that continues being represented by the paper surface) and (ii) moderate displacements of the $\mathrm{H}-\mathrm{H}$ axis out of the scattering plane. This leads to the following conclusions:

- Collisions where the internuclear axis is tilted with respect to $\mathbf{k}$ will correlate with small-medium impact parameters.

- Side-on collisions with medium-large impact parameters.

- Head-on collisions with large impact parameter.

It will be next proved that these relations reflect fairly good the connection between the reaction probability as a function of $J$ (total angular momentum quantum number) and the portraits tilting found for the state-to-state integer results. However, their employment requires to bear in mind that, in contrast to the $\mathrm{H}_{3}$ system (where the topology of the PES determines that, specially at low collision energies, the TS access is limited to few geometries around the linear minimum-energy geometry), the Stark and Werner PES is relatively flat in the TS region, involving that the $\mathrm{F}+\mathrm{H}_{2}$ reaction is less stereoselective with regard to the TS access and that our model can not be expected to work as properly as for the $\mathrm{H}+\mathrm{D}_{2}$ reaction.

Fig. 6.6 presents the reaction probability dependence on the total angular momentum quantum number for the $\mathrm{F}+\mathrm{H}_{2}(v=0, j=2)$ reaction at $E_{\text {coll }}=0.148 \mathrm{eV}$ and for different products states resolutions. The reaction probability functions $P(J)$ for state-to-state collisions are included in the middle $\left(v^{\prime}=2\right)$ and bottom $\left(v^{\prime}=3\right)$ panels of Fig. 6.6 and show that

- Reaction into low rotationally excited $\operatorname{HF}\left(v^{\prime}=2\right)$ molecules corresponds to a wide range of impact parameters where medium values predominate. As $j^{\prime}$ increases and the products get rotationally excited, the contribution from the 


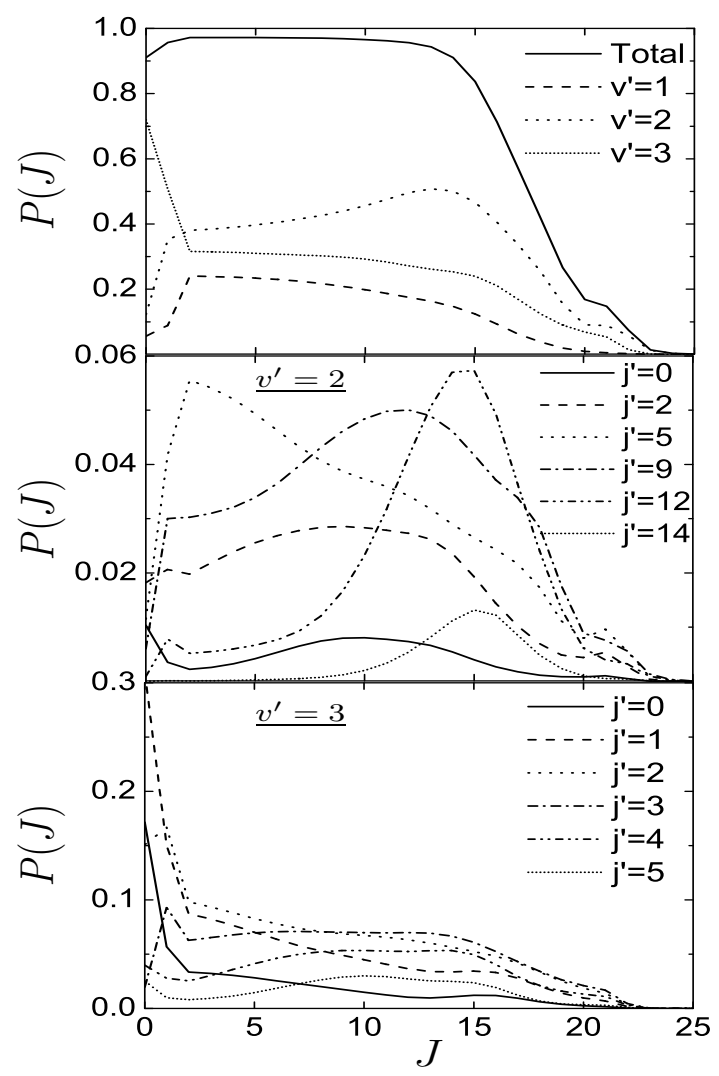

Fig. 6.6.: Total (top panel), vibrationally resolved (top panel) and state-to-state $\left(v^{\prime}=2\right.$ (medium panel) and $v^{\prime}=3$ (bottom panel) ) reaction probabilities as a function of the total angular momentum quantum number $(J)$ for $\mathrm{F}+\mathrm{H}_{2}(v=0, j=2)$ reactive collisions at $E_{\text {coll }}=0.148 \mathrm{eV}$. The $\mathrm{H}_{2}$ molecule is assumed to be initially unpolarised.

low impact parameters diminishes and the reaction goes mainly through large impact parameter collisions.

- The situation for $v^{\prime}=3$ collisions is slightly different. Low rotationally excited products are formed through collisions where the small-medium impact parameters contribution predominates. As $j^{\prime}$ increases this bias disappears and the reaction probability mainly concentrates at medium-large impact parameters.

According to this, the model predicts that collisions leading to $\operatorname{HF}\left(v^{\prime}=2\right)\left(\operatorname{HF}\left(v^{\prime}=\right.\right.$ 3)) will correlate with side-on (tilted) collisions when the products rotational excitation is low and for head-on (side-on) collisions when the rotational excitation of the 
products is high. This forecast is in good accordance with Figs. 6.3 and 6.4 internuclear axis portraits evolution, supporting the validity of the assumptions (access to TS geometry largely influences the collision geometry) underlying the model.

The change in the tendency for the internuclear axis to be located on the scattering plane is related to the reaction coplanarity: while $\mathrm{F}+\mathrm{H}_{2}$ reactive collisions leading to $v^{\prime}=3$ can be considered coplanar, this characteristic diminishes as $v^{\prime}$ decreases. Consistently, the state-to-state internuclear axis portraits always concentrate on the scattering plane when $\operatorname{HF}\left(v^{\prime}=3\right)$ is formed (Fig. 6.4) and display an increasing propensity toward out-of-plane geometries when the vibrational excitation of the products becomes lower (when $v^{\prime}=2$, this effect concentrates at medium values of $j^{\prime}$ (Fig. 6.3)).

To conclude this Section, the rotational portraits as a function of the products rotational excitation (middle of Figs. 6.3 and 6.4) will be considered. Their shape displays changes that can be classified into two groups: changes due to the $k$ even moments and already analysed by means of the internuclear axis portraits and changes related to the $k$ odd moments. In this last group, shape modifications resulting from $\mathrm{PP}\{1,1-\}$ sign inversion (indicating a switching of the $\mathbf{j}$ orientation along the $y$ axis) turns out to be particularly important: regardless HF vibrational excitation, low (high) values of $j^{\prime}$ correlate with fairly negative (positive) values of the moment, that is, with a noticeably rotational angular momentum orientation along the negative (positive) $y$ axis. This behaviour coincides with that found for the $\mathrm{H}+\mathrm{D}_{2}$ reaction and can be rationalised in the same way (see Fig. 5.5 and discussion therein): low (high) rotational excitation of the products correlates with collisions where the $\mathrm{F}$ and $\mathrm{H}$ atoms collide while moving along similar (opposite) direction, leading to less (more) vibrational excitation of the reaction complex which, in turn, leads to less (more) HF rotation.

\subsection{Vibrationally resolved differential results}

Explicit consideration of the scattering angle allows for determining how the mechanism changes with the products recoil direction and the extent of the DCS control that can be achieved through reactants polarisation. This Section copes with both issues for $\mathrm{F}+\mathrm{H}_{2}(v=0, j=2)$ reactive collisions where the products are detected without discriminating between different rotational states.

Figs. 6.7 and 6.8 consist of the differential cross section (summed over the final rotational levels) for $v^{\prime}=2$ and 3 together with internuclear axis portraits for selected values of the scattering angle in the backward and sideways regions (the analysis 


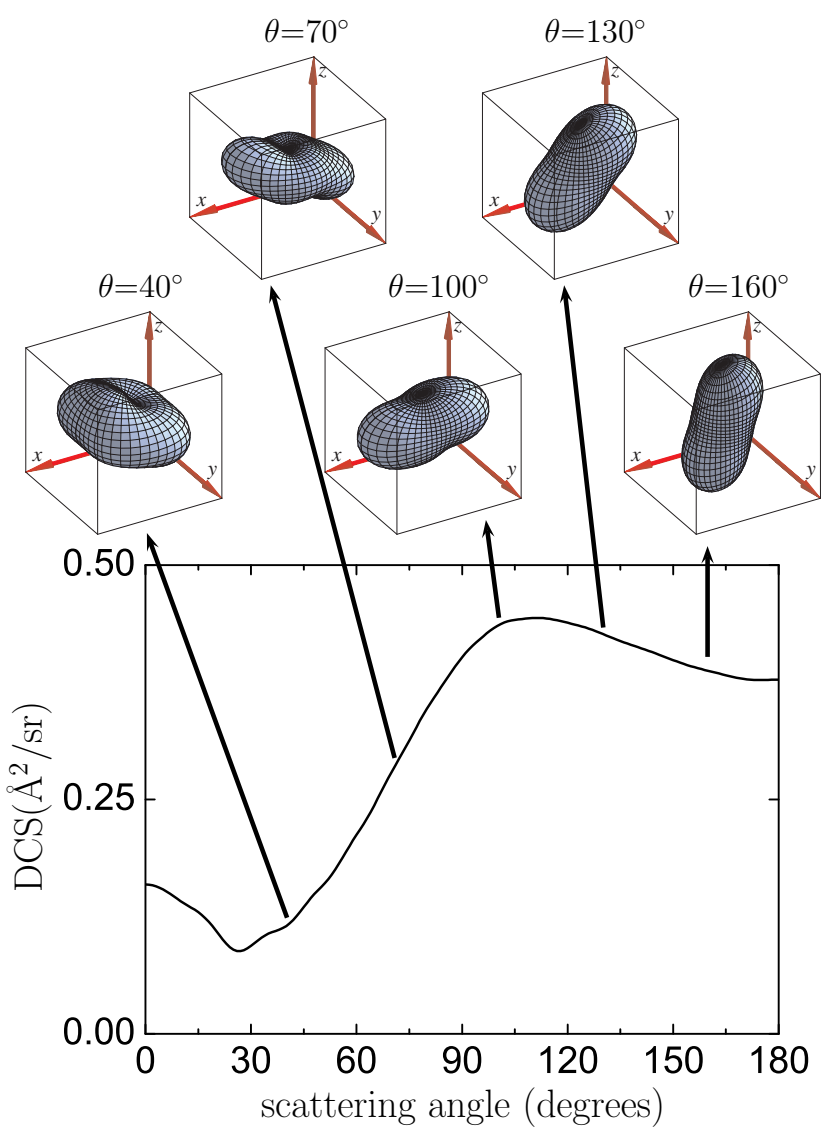

Fig. 6.7.: Differential cross section of the $\mathrm{F}+\mathrm{H}_{2}(v=0, j=2) \rightarrow \mathrm{HF}\left(v^{\prime}=2, j^{\prime}=\right.$ all $)+\mathrm{H}$ reaction at $E_{\text {coll }}=0.148 \mathrm{eV}$ and $\mathrm{H}_{2}$ internuclear axis stereodynamical portraits prepared using vibrationally resolved PDDCSs for the same reaction.

of the forward scattering will be separately performed). It is not worthy in this case to include rotational portraits as they do not provide with any information not contained in their internuclear axis counterparts, mainly because the vibrationally resolved renormalised PDDCS $\{1,1-\}$ cancels out.

It is interesting to point out that, in Fig. 7 of [121, the author presents $v^{\prime}$ resolved DCSs for the reaction of $\mathrm{F}$ with $\mathrm{n}-\mathrm{H}_{2}$ at the three Lee's energies. All the DCSs (but in particular those corresponding to $v^{\prime}=2$ and $E_{\text {coll }}=0.148 \mathrm{eV}$ ) present oscillations in the sideways region that are not reproduced by the DCSs in Figs. 6.7 and 6.8. This divergence can be attributed to the different maximum helicity quantum number used for the calculations (too small in [121]) and not to a physical effect. 


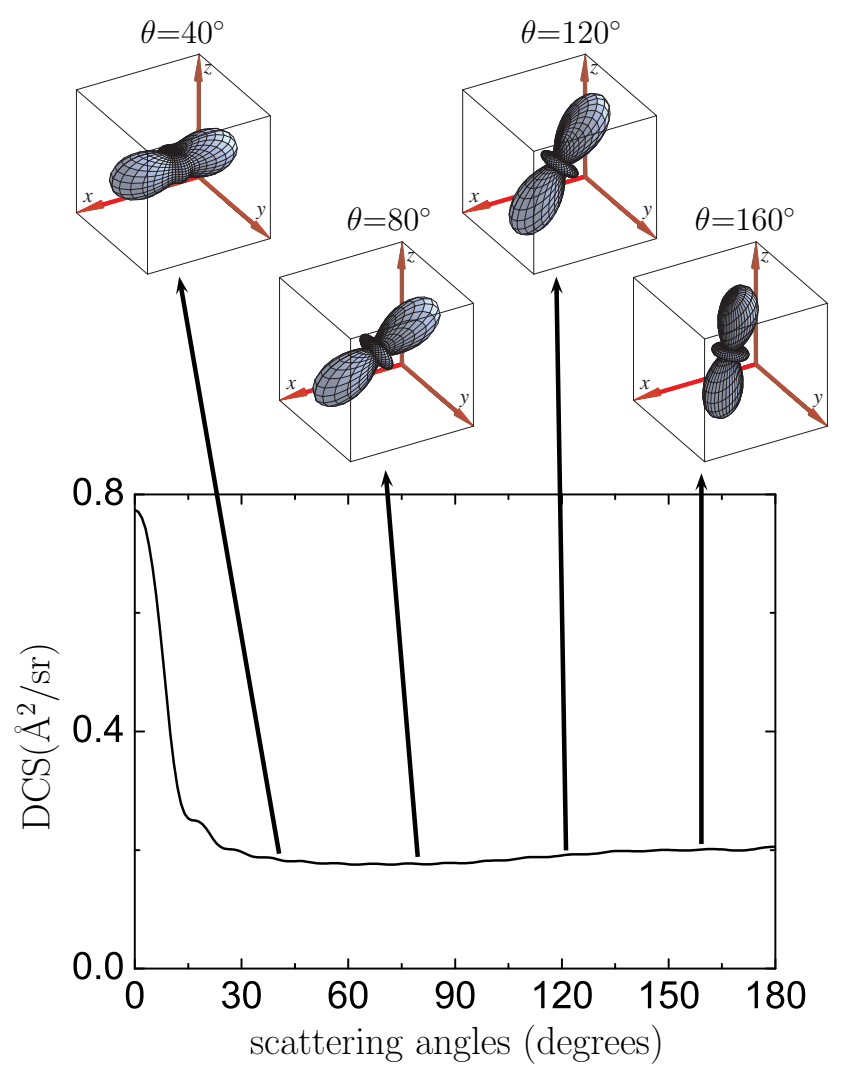

Fig. 6.8.: Differential cross section of the $\mathrm{F}+\mathrm{H}_{2}(v=0, j=2) \rightarrow \operatorname{HF}\left(v^{\prime}=3, j^{\prime}=\right.$ all $)+\mathrm{H}$ reaction at $E_{\text {coll }}=0.148 \mathrm{eV}$ and $\mathrm{H}_{2}$ internuclear axis stereodynamical portraits prepared using vibrationally resolved PDDCSs for the same reaction.

As expected, Fig. 6.7 shows that the DCS for $v^{\prime}=2$ concentrates on the backwardsideways region, displaying an small forward peak. It stems from the portraits analysis that:

- From $\theta \approx 70$ to $180^{\circ}$ the portraits tend to lie on the scattering plane, suggesting that the reaction happens through an approximately coplanar mechanism. For angles below $\approx 70^{\circ}$, the contribution from geometries where the axis is perpendicular to the scattering plane increases and the reaction can not be further described as coplanar.

- Backward scattering comes from head-on collisions. As $\theta$ decreases, the axis tilts in a progressive way, becoming perpendicular to $\mathbf{k}$ (preference for side-on 

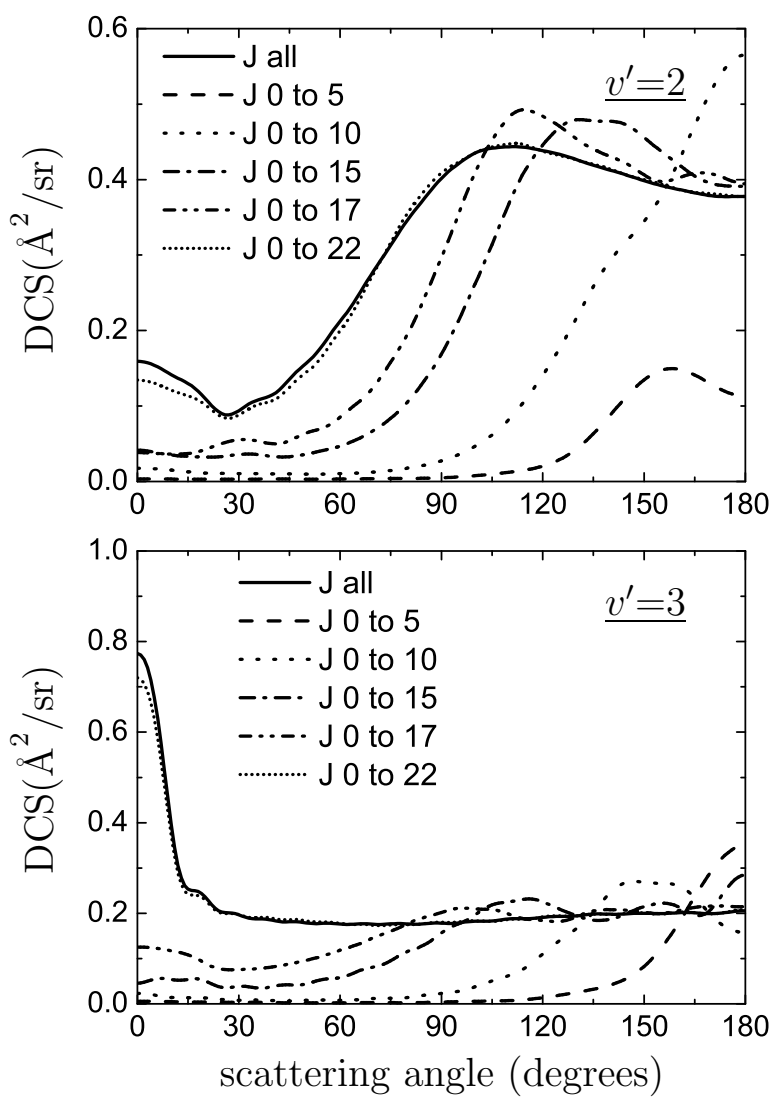

Fig. 6.9.: Building up of the $\mathrm{F}+\mathrm{H}_{2}(v=0, j=2) \rightarrow \mathrm{HF}\left(v^{\prime}=2,3, j^{\prime}=\right.$ all $)+\mathrm{H}$ reaction differential cross sections at $E_{\text {coll }}=0.148 \mathrm{eV}$. Top panel corresponds to $v^{\prime}=2$ and bottom panel to $v^{\prime}=3$.

colisions) at $\theta \approx 100^{\circ}$.

Fig. 6.8 displays the famous $v^{\prime}=3$ forward peak that sticks out over the approximately constant sideways-backward DCS. The analysis of the portraits shows that:

- The portraits are slimmer and more concentrated on the scattering plane than their $v^{\prime}=2$ counterparts, indicating a larger specificity of the directional requirements for reaction. Consistently with its coplanar nature, reactive collisions leading to $\operatorname{HF}\left(v^{\prime}=3\right)$ tend to happen with the internuclear axis located on the scattering plane for the whole $\theta$ interval considered (backward-sideways).

- Backward scattering comes again from head-on collisions. As $\theta$ decreases, the angle between $\mathbf{r}$ and $\mathbf{k}$ increases progressively (rotation of the portrait as the 
scattering angle changes is slower than for $v^{\prime}=2$ ), with $\mathbf{r}$ becoming perpendicular to $\mathbf{k}$ at $\theta \approx 40^{\circ}$.

It is necessary now to ascertain whether these findings can be rationalised through the model used for the integral state-to-state results or not. To this purpose, we performed a building-up analysis of the $v^{\prime}=2$ and 3 DCSs (Fig. 6.9) that furnished us with the following information

- The largest contribution to the backward DCS comes from the small-medium (small predominate when $v^{\prime}=3$ and medium when $v^{\prime}=2$ ) $\mathbf{J}$ values. However, to fully converge the backward scattering it is necessary to take into account most of the partial waves (20 at least).

- Sideways DCS comes from medium-large $\mathbf{J}$ values. As the scattering angle gets closer to the backward (forward) region, medium (large) impact parameters become predominant.

- Most of the forward scattering proceed from the largest values of $\mathbf{J}(17-25)$ (although not all the reaction probability corresponding to these $\mathbf{J}$ values ends up as forward scattering).

Considering the contribution of the different partial waves, the model predictions do not fully agree with the portraits behaviour in Figs. 6.7 and 6.8. According to the model, the most favourable collision geometry would change with the scattering angle as follows: $\mathbf{r}$ will display a preference for being $\approx 45^{\circ}$ tilted with respect to $\mathbf{k}$ for scattering angles close to $180^{\circ}$ (small impact parameters are important), changing into a preference for side-on collisions when the scattering angle starts decreasing (medium impact parameters predominate) and finishing with a tendency toward headon collisions for the sideways region closer to forward scattering (products formed at these scattering angles arise from large impact parameter collisions). It is evident that there is a noticeable disagreement between this forecast and the evolution displayed by the portraits in Figs. 6.7 and 6.8 .

Two reasons can be put forward in order to justify such difference:

- Scattering at 0 and $180^{\circ}$ displays axial symmetry around $\mathbf{k}$ and, in consequence, the stereodynamical portraits must display such symmetry as well. The direct (responsible for the backward-sideways scattering) mechanism is characterised by gradual changes of the intrinsic polarisation moments and of the corresponding portraits, involving that the shapes of the portraits for scattering angles in the backward region will be constrained by the "compulsory" axial symmetry at $180^{\circ}$. 


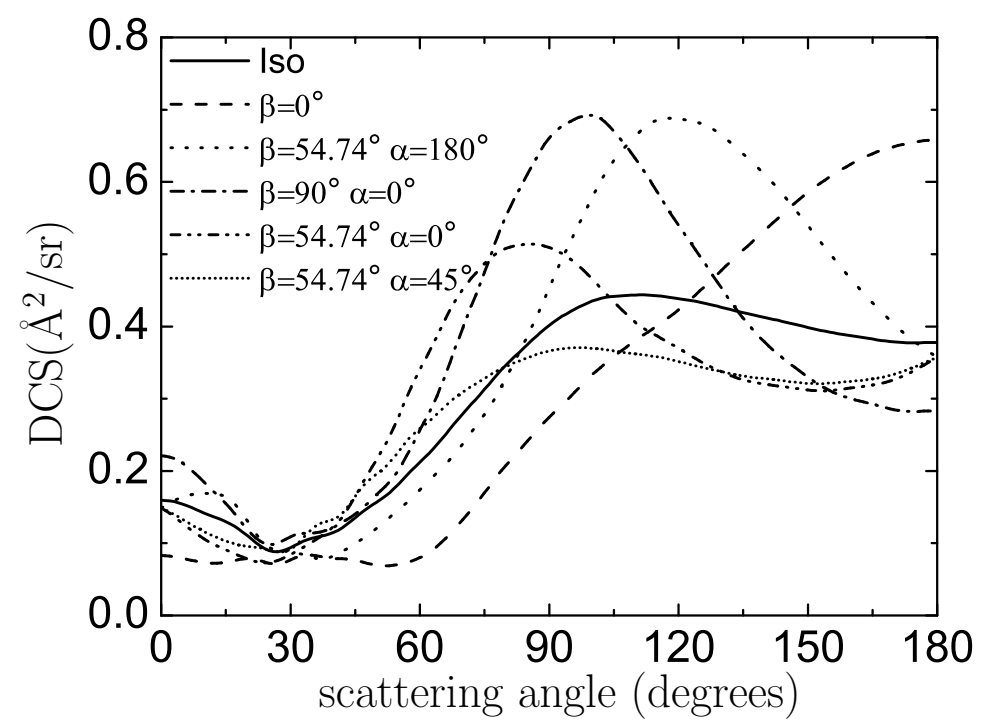

Fig. 6.10.: Differential cross section of the $\mathrm{F}+\mathrm{H}_{2}(v=0, j=2) \rightarrow \operatorname{HF}\left(v^{\prime}=2, j^{\prime}=\right.$ all) $+\mathrm{H}$ reaction at $E_{\text {coll }}=0.148 \mathrm{eV}$ for unpolarised reactants (Iso) and different $\mathrm{H}_{2}$ alignment directions.

- The TS region of the SW PES has a relatively flat character, giving rise to a lack of stereoselectivity for accessing that part of the surface. This fact remained concealed during the integral results discussion and, only through the explicit consideration of the scattering angle in the differential results, it has been unveiled.

The intrinsic internuclear axis portraits for $v^{\prime}=2$ and 3 in the backward region (Figs. 6.7 and 6.8) are quite similar to the extrinsic axis preparation corresponding to $\beta=0^{\circ}$ (see Fig. 4.4). As the scattering angle decreases, the intrinsic portraits tilt without noticeable shape distortions (except in the sideways $v^{\prime}=2$ region). This two facts suggest that one can control the backward and sideways DCS through the experiment proposed in Chapter 4 just by moving the alignment direction in the $\left(0^{\circ}, 120^{\circ}\right) \beta$-interval with $\alpha=180^{\circ}$. The control will manifest through the raising of a DCS peak whose center (corresponding to the scattering angle where the intrinsic and the extrinsic portraits are more similar) can be shifted at one's discretion by changing the tilting of the alignment direction with respect to $\mathbf{k}$.

Such effect can be illustrated through Figs. 6.10 and 6.11 , which present the $v^{\prime}=2$ and 3 DCSs for unpolarised and polarised reactants. The extrinsic preparations employed to generate those Figures can be classified into two different groups: 


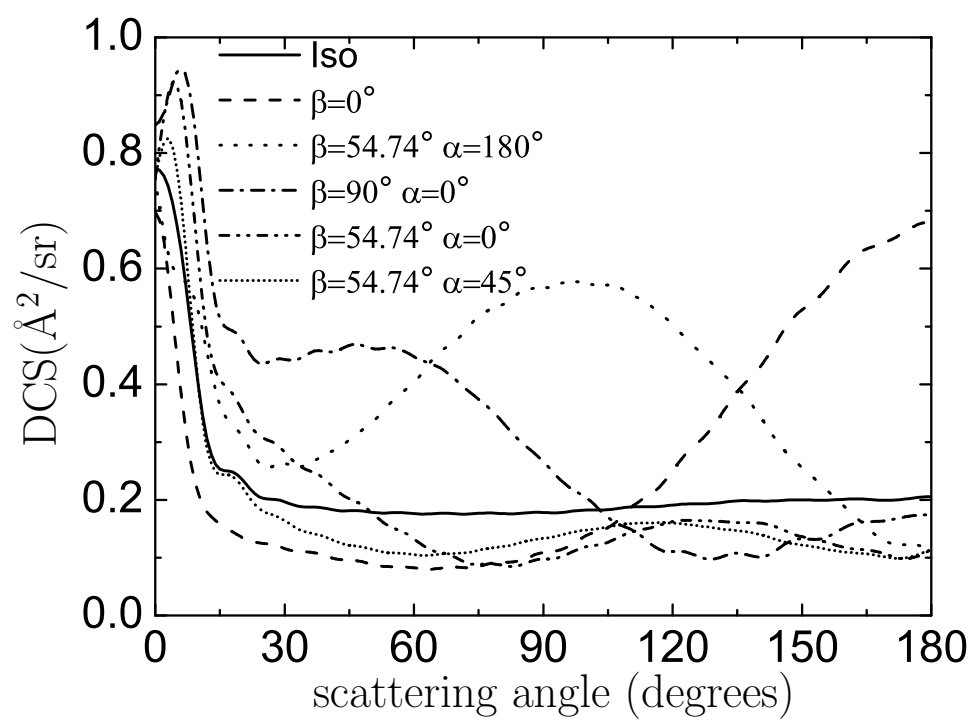

Fig. 6.11.: Differential cross section of the $\mathrm{F}+\mathrm{H}_{2}(v=0, j=2) \rightarrow \operatorname{HF}\left(v^{\prime}=3, j^{\prime}=\right.$ all) $+\mathrm{H}$ reaction at $E_{\text {coll }}=0.148 \mathrm{eV}$ for unpolarised reactants (Iso) and different $\mathrm{H}_{2}$ alignment directions.

- Preparations where the internuclear axis is on the scattering plane: $\beta=0^{\circ}$, $\beta=54.74^{\circ} \alpha=180^{\circ}, \beta=90^{\circ} \alpha=0^{\circ}$ and $\beta=54.74^{\circ} \alpha=0^{\circ}$ (the corresponding internuclear axis and rotational extrinsic portraits are included in Fig. 4.4).

- Preparation where the internuclear axis is out of the scattering plane: $\beta=54.74^{\circ}$ $\alpha=45^{\circ}$.

Regarding $v^{\prime}=3$ (Fig. 6.11), head-on $\left(\beta=0^{\circ}\right)$ collisions completely modify the DCS shape. This extrinsic preparation slightly reduces the forward DCS, reduces the sideways DCS and triples the backward DCS, giving rise to a noticeable peak at $180^{\circ}$. This peak shifts to the left as we tilt the axis preparation: for $\beta=54.74^{\circ}, \alpha=180^{\circ}$ the peak appear at $\theta=100^{\circ}$ and for $\beta=90^{\circ}, \alpha=0^{\circ}$ appears at $\theta \approx 50^{\circ}$. Preparations where the internuclear axis is out of the scattering plane (for instance $\beta=54.74^{\circ}$, $\alpha=45^{\circ}$ ) provoke a general reduction of the DCS. In contrast to the backward-sideways DCS, the forward peak turns out to be quite insensitive to this kind of preparations. Discussion of Fig. 6.10 can be (grosso modo) performed in the same terms: head-on collisions reduce the forward and the sideways DCSs and increases the backward DCS, giving rise to a $180^{\circ}$ peak which shifts to the left as its $v^{\prime}=3$ counterpart. There are, however, three differences between the results for both vibrational levels: 


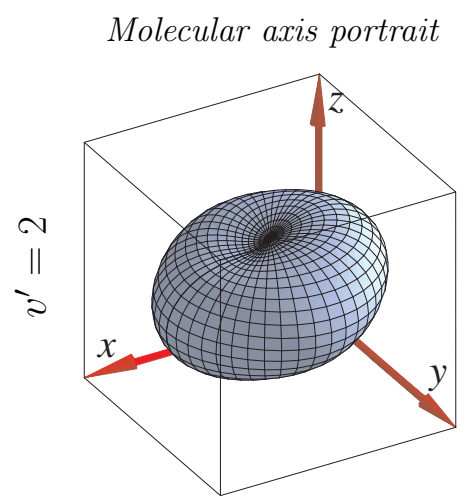

Rotational portrait

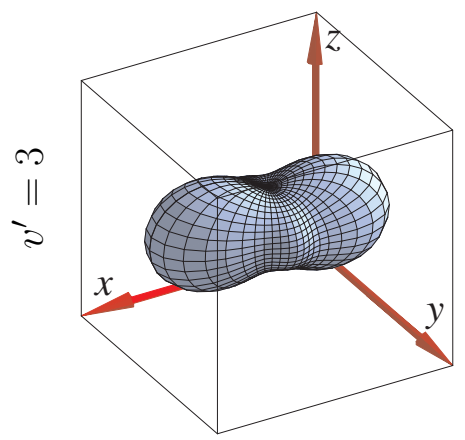

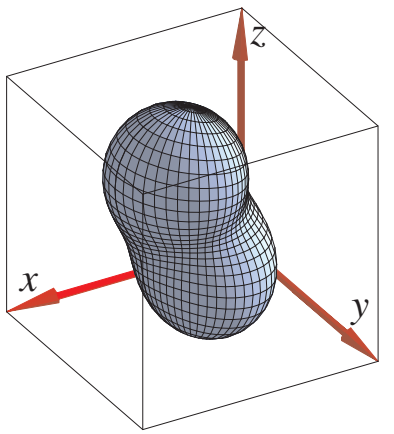

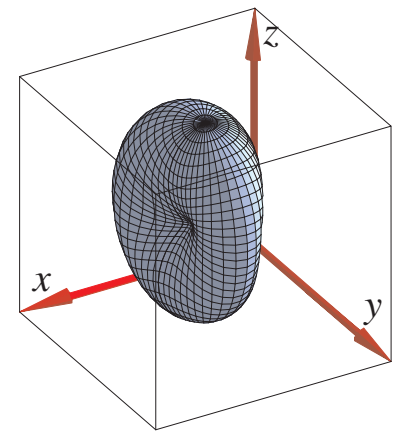

Fig. 6.12.: Intrinsic $\mathrm{H}_{2}$ stereodynamical portraits representing the $\mathbf{r}$ and $\mathbf{j}$ distributions giving rise to $v^{\prime}=2$ (top) and 3 (bottom) forward scattering at $E_{\text {coll }}=0.148 \mathrm{eV}$.

- Forward scattering is, in relative terms, more sensitive to the extrinsic preparation of the reactants when $v^{\prime}=2$ than when $v^{\prime}=3$.

- The intrinsic portraits for $v^{\prime}=3$ are more similar to the extrinsic distributions than their $v^{\prime}=2$ counterparts. This explains why the control of the DCS that can be achieved by extrinsically aligning $\mathbf{j}$ is larger for $v^{\prime}=3$ than for $v^{\prime}=2$.

- While preparations where the internuclear axis is not located on the scattering plane hinder the $v^{\prime}=3$ scattering all along the backward-sideways region, this is not the case for $v^{\prime}=2$ as such preparations slightly favour the reaction at scattering angles around $65^{\circ}$. The analysis of the intrinsic portraits in Fig. 6.7 justifies this feature because the portraits corresponding to those scattering angles do not concentrate on the scattering plane but stick out from it.

The nature of the mechanism leading to forward scattering, whose analysis has been skipped so far, will be discussed in the following Section, devoted to the state- 
to-state differential results. It is possible, however, to perform now a first approach to its study by considering the polarisation moments obtained by averaging ${ }^{11}$ the $v^{\prime}=2$ and 3 PDDCSs in the forward region (from 0 to $20^{\circ}$ ) and the corresponding portraits (Fig. 6.12), which show that

- There are noticeable differences between the mechanism leading to $v^{\prime}=2$ and 3 forward scattering.

- For $v^{\prime}=2$, both portraits almost display azimuthal symmetry around $\mathbf{k}$. The internuclear axis portrait shows that side-on collisions are preferred.

- For $v^{\prime}=3$, collisions leading to forward scattering are more stereoselective. The internuclear axis distribution is fairly flat (its plane coincides with the scattering plane) and perpendicular to $\mathbf{k}$ (preference for side-on collisions). The rotational angular momentum is strongly aligned along $\mathbf{k}$ and slightly oriented along $-\hat{y}$.

Attentive readers can put forward that the shapes of these portraits are not a surprise but the natural evolution of those included in Figs. 6.7 and 6.8 and, therefore, they do not support the fact that the mechanism giving rise to forward scattering differs from that responsible for sideways and backward reaction. This point will be next clarified.

\subsection{State-to-state differential results}

A comprehensive and very illustrative way of visualising the influence of the reactants polarisation on the DCS is provided by the polar maps included in Fig. 6.13 In particular, they make possible to ascertain (up to a limit given by the resolution of the figure and by the number and population of the opened products channels) how that influence manifests for the different HF internal states:

- The isotropic polar map (unpolarised reactants) displays four groups of rings, although the inner two $\left(v^{\prime}=2\right.$ and 3$)$ concentrate most of the reaction probability. As expected, the $v^{\prime}=2$ rings mainly correspond to sideways and backward scattering and the $v^{\prime}=3$ rings show the well known forward peak (together with an approximately constant sideways-backward DCS).

${ }^{11}$ Intrinsic polarisation moments corresponding to a well defined interval of scattering angles $\left[\theta_{\min }, \theta_{\max }\right]$ can be defined as follows

$$
s_{q}^{\{k\}}\left[\theta_{\min }, \theta_{\max }\right]=\frac{\int_{\theta_{\min }}^{\theta_{\max }} S_{q}^{\{k\}}(\theta) \sin \theta d \theta}{\int_{\theta_{\min }}^{\theta_{\max }} S_{0}^{\{0\}}(\theta) \sin \theta d \theta}
$$

The polarisation parameters are nothing but an special case of this expression: $s_{q}^{\{k\}}[0, \pi]$ 

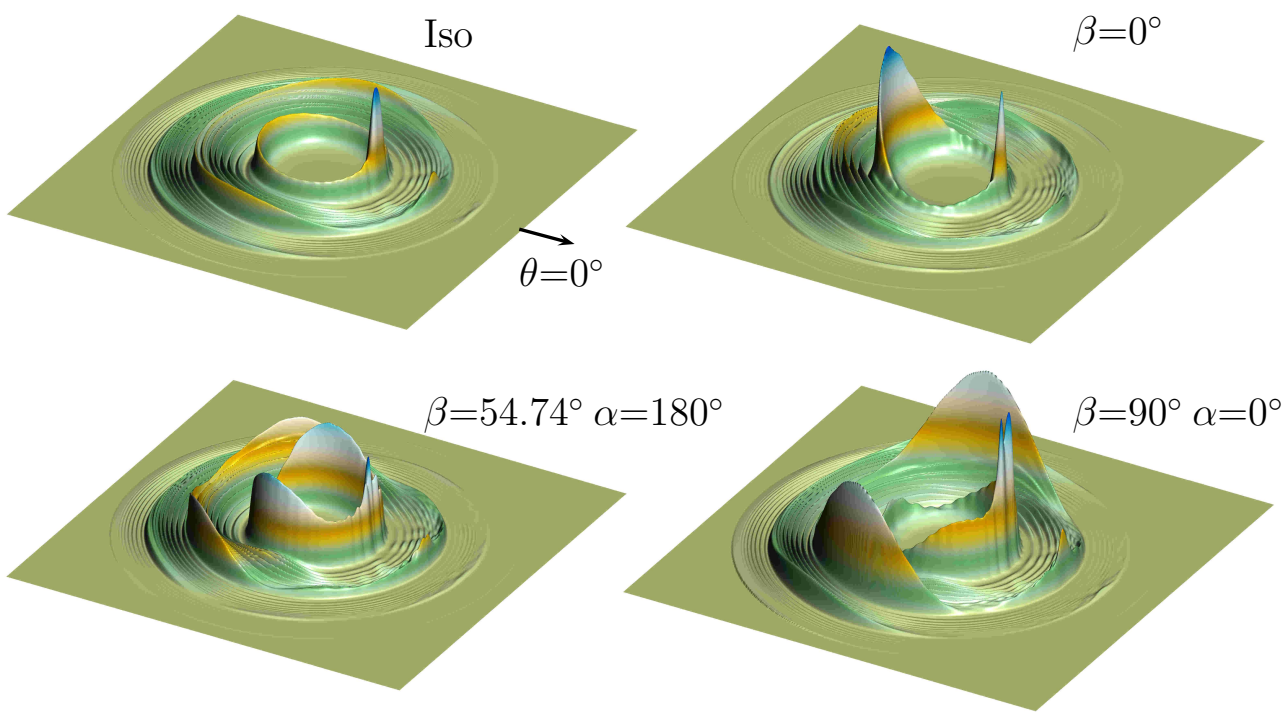

Fig. 6.13.: Quantum triple (angle-velocity) differential cross section (polar maps) of the $\mathrm{F}+\mathrm{H}_{2}(v=0, j=2)$ reaction at $E_{\text {coll }}=0.148 \mathrm{eV}$ and for different $\mathrm{H}_{2}$ alignment directions.

- Head-on collisions ( $\beta=0^{\circ}$ preparation) have been found to boost (reduce) backward (sideways) scattering. The analysis of the corresponding polar map furnishes with a deeper insight into this reaction feature: for collisions leading to $v^{\prime}=2$, such increment in the backward DCS is mainly due to formation of products characterised by medium-high values of $j^{\prime}$.

- Regarding $\beta=54.74^{\circ}, \alpha=0^{\circ}$ and $\beta=90^{\circ}, \alpha=180^{\circ}$ preparations, they originate a noticeable augment of sideways scattering (with the differences indicated in the former Section). When $\beta=54.74^{\circ}, \alpha=0^{\circ}$ and $v^{\prime}=2$, this increment distributes over all values of $j^{\prime}$, giving rise in each case to formation of a peak that shifts into smaller scattering angle regions as $j^{\prime}$ increases. On the contrary, when $\beta=90^{\circ}, \alpha=0^{\circ}$ and $v^{\prime}=2$, the increment concentrates on low rotationally excited products, which triple their isotropic DCS value.

To perform an exhaustive analysis of the state-to-state reaction stereodynamics in the backward-sideways region is extremely cumbersome and does not shed additional light on the corresponding reaction mechanism. On the contrary, its consideration on the forward region unveils the real nature of the collisions mechanism leading to forward scattering. 

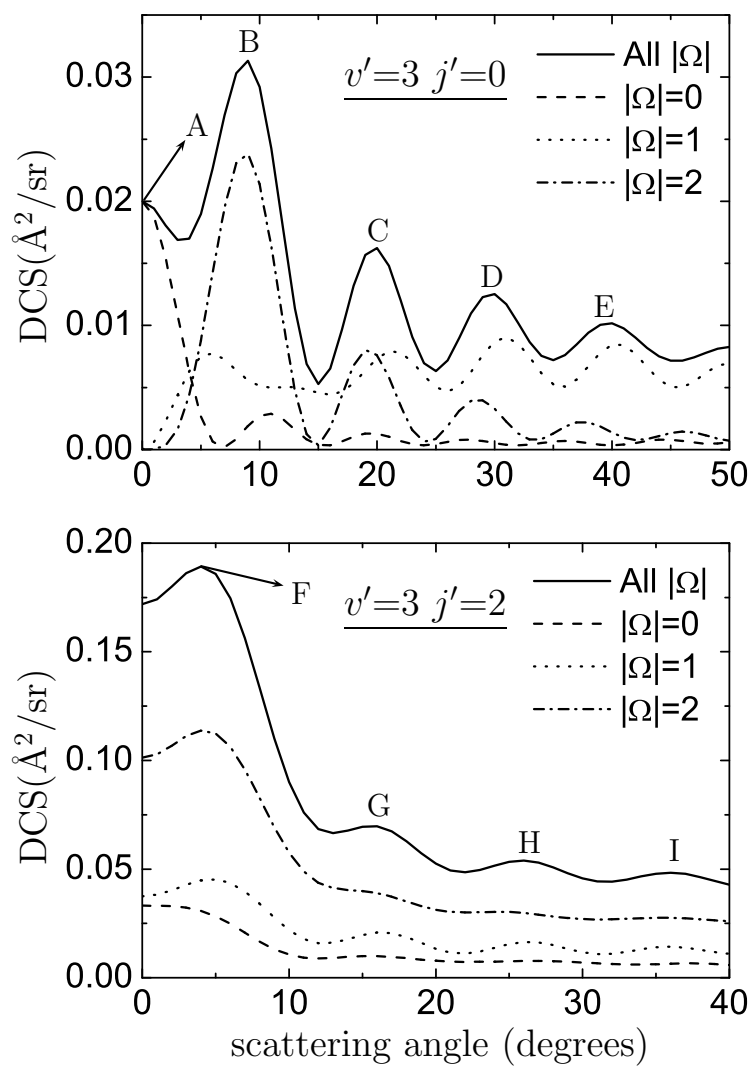

Fig. 6.14.: Differential cross sections, in the forward scattering region, of the $\mathrm{F}+\mathrm{H}_{2}(v=0, j=2) \rightarrow \mathrm{HF}\left(v^{\prime}=3, j^{\prime}=0,2\right)+\mathrm{H}$ reactions at $E_{\text {coll }}=0.148$ $\mathrm{eV}$. The solid line represents the full result; the other lines represent partial contributions from selected $\mathrm{H}_{2}$ helicities.

With this purpose, let us select the following two state-to-state processes

$$
\mathrm{F}+\mathrm{H}_{2}(v=0, j=2) \rightarrow \mathrm{HF}\left(v^{\prime}=3, j^{\prime}=0,2\right)+\mathrm{H}
$$

whose forward DCS, together with the decomposition into partial contributions from absolute values of the $\mathrm{H}_{2}$ helicity, are presented in Fig. 6.14 and whose $k=1$ and 2 renormalised PDDCSs are plotted in Fig. 6.15.

The nearside-farside analysis of $\mathrm{F}+\mathrm{H}_{2}(v=0, j=1)$ collisions performed by Connor and co-workers [115] showed that

- The main contribution to sideways and backward scattering comes from nearside collisions. 

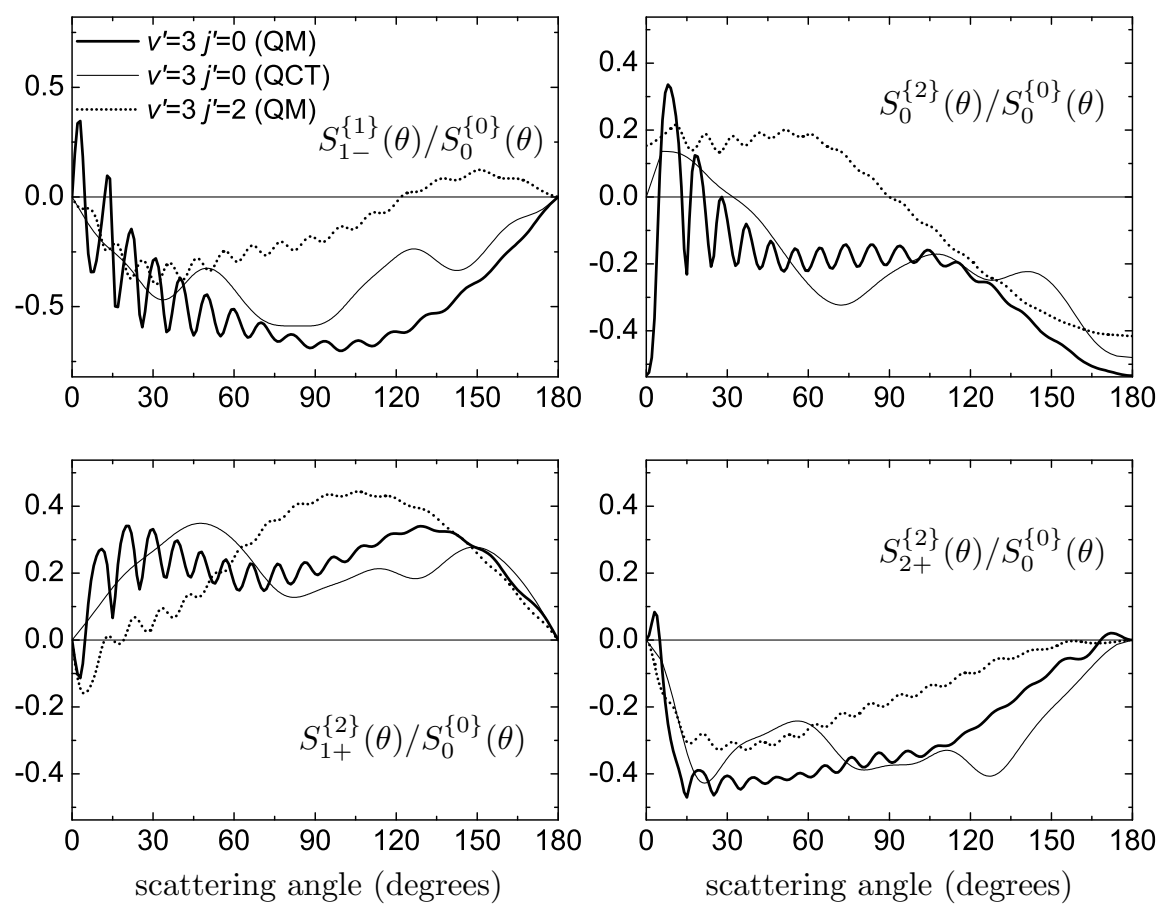

Fig. 6.15.: $k=1$ and 2 renormalised state-to-state PDDCSs for the $\mathrm{F}+\mathrm{H}_{2}(v=0, j=$ $2) \rightarrow \operatorname{HF}\left(v^{\prime}=3, j^{\prime}=0,2\right)+\mathrm{H}$ reactions at $E_{\text {coll }}=0.148 \mathrm{eV}$. Results for $v^{\prime}=3, j^{\prime}=0$ include moments evaluated using QM (thick continuous line) and QCT (thin continuous line), while for $v^{\prime}=3, j^{\prime}=2$ only QM (dotted line) calculations are presented.

- Forward $\left(\theta\right.$ between 0 and $\left.30^{\circ}\right)$ comes from the interference between nearside and farside scattering (the magnitude of farside is comparable to that of nearside only for the angles closer to $0^{\circ}$ ).

These findings suggest that the results included in Figs. 6.14 and 6.15 will not cualitatively differ from their $\mathrm{H}+\mathrm{D}_{2}$ counterparts (see Chapter $\underline{5}$ ), displaying oscillations in the forward region caused by nearside-farside interferences, and justify why the model proposed to rationalise the state-to-state results (Sec. 6.4) was devised in terms of nearside collisions.

The analysis of Fig. 6.14 is in accordance with Fig. 5.11 for the $H+\mathrm{D}_{2}$ reaction. As expected, we find the same oscillations in the $|\Omega|$ resolved DCSs. The existence of such oscillations is not concealed by summation over the helicity values and, in fact, they are responsible for the appearance of a succession of peaks in the "whole" DCS 
- $v^{\prime}=3 j^{\prime}=0 \rightarrow$ peak A comes from $|\Omega|=0$ (there is no other chance in this case), peak B from $|\Omega|=2$ and peaks $\mathrm{C}, \mathrm{D}$ and $\mathrm{E}$ from $|\Omega|=1$ and 2 (where the contribution of $|\Omega|=1$ (2) becomes predominant (vanishes) as $\theta$ moves away from $\left.0^{\circ}\right)$.

- $v^{\prime}=3 j^{\prime}=2 \rightarrow$ peak $\mathrm{F}$ can be attributed to $|\Omega|=2$ (and in some extent also to 1 ) and peaks $\mathrm{G}, \mathrm{H}$ and I to $|\Omega|=1$ oscillations.

The amplitude of the $|\Omega|$ resolved DCSs oscillations diminishes as $j^{\prime}$ increases and, if a partial wave decomposition is performed, they are found to arise from the interference between partial waves corresponding to large values of $J$ (it is not possible to set a one-to-one correspondence between peaks and partial waves but, on the contrary, all large $J$ values contribute to all forward peaks).

The renormalised PDDCSs (Fig. 6.15), with the familiar QM oscillations in the forward region, turn out to be more interesting than the DCS. A fleeting glance at the plots shows that

- The origin of the oscillations is unequivocally quantum mechanical, as it can be seen by comparing the QM PDDCSs for $\left(v^{\prime}=3, j^{\prime}=0\right)$ with its QCT counterpart. This is not a surprise, as there is no room for undulatory effects (like the nearside-farside interferences) in classical mechanics.

- The amplitude of the oscillations decreases as $j^{\prime}$ increases and, at a fixed value of $j^{\prime}$, such amplitude is always smaller than for the $\mathrm{H}+\mathrm{D}_{2}$ reaction (see Fig. 5.8). In fact, the plots of the QM PDDCSs reminds the kinematics studied during the high school and, in particular, the superposition of two perpendicular movements: an oscillatory movement of small amplitude and another "principal" (large amplitude) movement whose trajectory is perturbed by the first one in such a way that the resultant trajectory displays oscillations. In terms of the mechanism, the principal movement is tantamount to the nearside collisions and the secondary one to the farside collisions, which do not play any role at all for backward scattering and become more and more significant as the scattering angle goes through the sideways region and get closer to $0^{\circ}$. This could be interpreted by saying that the delayed mechanism, responsible for the forward scattering, is nothing but the nearside collisions mechanism (the direct mechanism) perturbed by the appearance of reactive farside collisions.

- This perturbation is not, however, as important as for the $\mathrm{H}+\mathrm{D}_{2}$ reaction. One consequence of the relatively small amplitude of the PDDCSs oscillations is that, if the $\mathrm{F}+\mathrm{H}_{2}$ PDDCSs are integrated over the scattering angle in the forward 

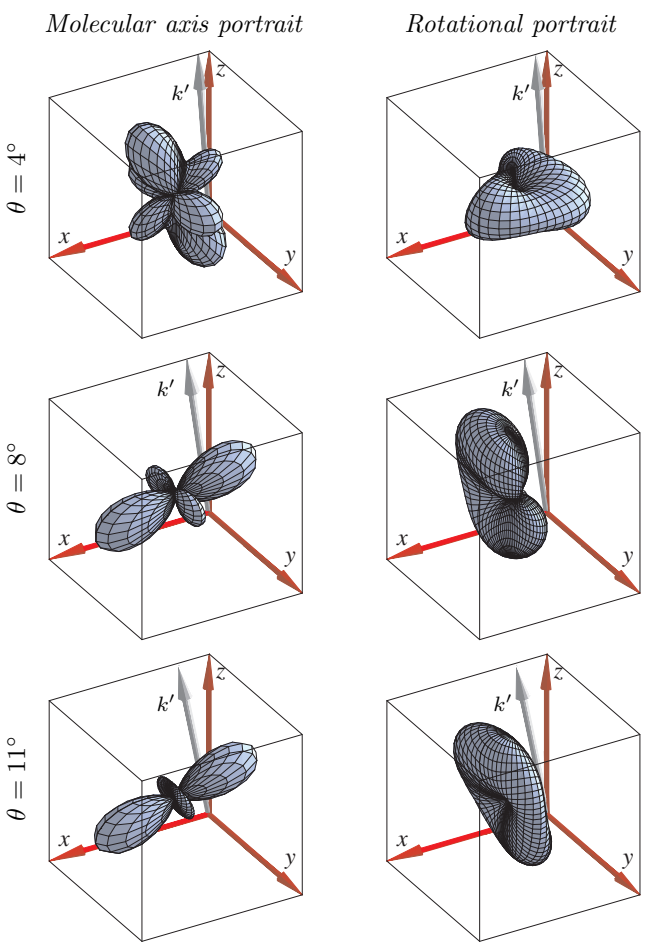

Fig. 6.16.: Stereodynamical portraits of reaction $\mathrm{F}+\mathrm{H}_{2}(v=0, j=2) \rightarrow \operatorname{HF}\left(v^{\prime}=\right.$ $\left.3, j^{\prime}=0\right)+\mathrm{H}$ at $E_{\text {coll }}=0.148 \mathrm{eV}$, and with $\theta=4^{\circ}$ (top), $\theta=8^{\circ}$ (middle), or $\theta=11^{\circ}$ (bottom). The portraits on the left (right) column show the distributions of the $\mathrm{H}_{2}$ interatomic axis (rotational angular momentum) when the reaction happens. The scattering angle $\theta$ between the reactantapproach and product-recoil directions is the angle between the vectors $\mathbf{k} \equiv z$ and $\mathbf{k}^{\prime}$.

region, the results would mainly represent the characteristics of the nearside collisions because, in a large extent, the farside contributions cancel out (see Fig. 6.12 and the comments therein).

- The interval of scattering angles where the oscillations can be distinguished is broader than for the $\mathrm{H}+\mathrm{D}_{2}$ reaction. This could indicate that the complex formed immediately after the collision survives for longer and has more time to rotate before breaking, taking to larger time delays in QCT or wavepacket studies (the results included in ref [137], although for the F+HD isotopic variant of the reaction, seem to support this statement). 
The effect of the PDDCSs oscillations on the reaction stereodynamics can be appreciated as well through consideration of the scattering angle resolved portraits presented in Fig. 6.16. We found again that, due to such oscillations, small variations in the scattering angle largely modifies the shape of the portraits. However, important as these changes are, their extent is smaller than that found for the $\mathrm{H}+\mathrm{D}_{2}$ reaction (compare with Fig. 4.6) and concentrates at angles closer to $0^{\circ}$, where the importance of farside collisions is larger and the PDDCSs oscillations are more intense. 


\section{Mechanism and control of atom-diatom reactions at low and ultralow collision energies}

The dependence on stereodynamical factors of the mechanism and reactivity of the atom-diatom reactions at low and ultralow collision energies is now studied. The results will be illustrated by means of examples corresponding to the benchmark $\mathrm{F}+\mathrm{H}_{2}$ reaction, where the impact of polarisation of the $\mathrm{H}_{2}$ rotational angular momentum on total and state-to-state integral and differential cross sections is analysed, leading to:

- Detailed pictures of the reaction mechanism in the cold and ultracold regimes, accounting in particular for distinctions associated with the various product states and scattering angles.

- Assessment of the extent to which selection of reactant polarisation allows for external control of the reactivity. This reveals that even the simplest of reactant polarisation schemes allows for fine, product state-selective control of differential and (for reactions involving more than a single, zero orbital angular momentum partial wave) integral cross sections.

The reasons for the interest in ultracold chemical reactions have been discussed in recent reviews [138, 139]. The usage of electric and magnetic fields open the door for interesting control possibilities [138, 140, 141, 142, 143, 144, 145, 146] that would not be possible at larger temperatures. The basic idea is that, at very low collision energies, the perturbation induced by the external field is larger than the collision energy and can be used to control the collision output. For instance, it is possible (i) to alter the effect upon the reaction of centrifugal barriers in the final reaction channels [140, (ii) to modify the intermolecular forces between polar molecules [141, 144, in such a way that the stability of complexes formed by these molecules can be controlled, (iii) to study collisions coupling electronic states which, if the spherical symmetry of 
the problem were not broken by the external field, would be uncoupled [143] and (iv) to open or to close reaction channels [146].

The Chapter begins by defining cold and ultracold reactions and by characterising their scattering properties in the zero collision energy limit (Sec. 7.1). It continues with a survey of the intrinsic moments behaviour in the ultracold regime, providing us with a general overview of the ultracold atom-diatom reactions control possibilities (Sec. 7.2). These results will be illustrated by studying the stereodynamics of the $\mathrm{F}+\mathrm{H}_{2}(v=0, j=2)$ reaction at low and ultralow collision energies (Sec. 7.3): after briefly describing how the scattering matrices for the selected energies were worked out (Sec. 7.3.1), we will analyse the reaction mechanism (Sec. 7.3.2) and the extent of the control achievable by suitably polarising the $\mathrm{H}_{2}$ molecule (Sec. 7.3.2).

\subsection{Cold and ultracold reactions}

Some authors (see [147]) define ultracold molecules as those whose translational energy is no larger than $1 \mathrm{~cm}^{-1} \approx 120 \mu \mathrm{eV}$. Others define ultracold molecules as those whose translational temperature is below $1 \mathrm{mK}$ (see [138), which implies $E_{\text {trans }} \leq$ $0.1 \mathrm{\mu eV}$. Therefore, whether the considered energies of interest qualify a collision as ultracold or not is, to some extent, a matter of opinion. In what follows, we shall restrict the "ultracold" qualifier to collision energies at which scattering cross sections are entirely due to $s$-wave scattering ${ }^{1}$. While generally accepted [138, 139], this definition suffers from the disadvantage that the ultracold threshold must be determined theoretically and changes from system to system. Cold collision energies will be those which, although small, involve contributions to reactivity from a few $l \neq 0$ partial waves.

The chemical reactions rate coefficient is independent of temperature and finite in the limit $E_{\text {coll }} \rightarrow 0$, where only reactive collisions with $l=0$ give rise to products formation. The justification for this assertion ensues from the behaviour of the reactive cross section in the zero collision energy limit which, according to Wigner [148], is given by

$$
\lim _{E_{\text {coll } \rightarrow 0}}(\sigma) \propto E_{\text {coll }}^{-1 / 2}
$$

When Eq. (3.6) (linking rate coefficients with reactive cross section values) is combined with this expression, it becomes simple to prove that the rate coefficient becomes

${ }^{1}$ Only the $l$ (orbital angular momentum quantum number) zero partial wave contributes to the process 
independent on the temperature

$$
\begin{array}{r}
k(T)=\left(\frac{\mu}{2 \pi k_{B} T}\right)^{3 / 2} \int_{0}^{\infty} v_{r} \sigma\left(v_{r}\right) \exp ^{-\mu v_{r}^{2} / 2 k_{B} T} 4 \pi v_{r}^{2} d v_{r} \\
E_{\text {coll } \rightarrow 0} \underbrace{\frac{1}{\left(k_{B} T\right)^{3 / 2}} \underbrace{\int_{0}^{\infty} E_{\text {coll }} \overbrace{\sigma\left(E_{\text {coll }}\right)}^{\operatorname{Eq}((7.1)} \exp ^{-E_{\text {coll }} / k_{B} T} d E_{\text {coll }}}_{\left(k_{B} T\right)^{3 / 2}}}_{T \text { independent }}
\end{array}
$$

where $E_{\text {coll }}=\mu v_{r}^{2} / 2$, the relationship between collision energy and reactants relative velocity, has been employed. The constant value of the ultracold rate coefficient can eventually take significatively large values (this happens, for instance, for $\mathrm{F}+\mathrm{H}_{2}$ [147, 149]).

\subsection{Control of $\ell=0$ reactions}

\subsubsection{Ultracold reactions and $S$ matrix}

The transformation between the orbital angular momentum $(l)$ and helicity $(\Omega)$ representations of the scattering matrix is given by Eqs. (3.39) and (3.40), which can be combined into the expression

$$
S_{j^{\prime} \Omega^{\prime}, j \Omega}^{J P}=\frac{2}{\left(1+\delta_{\Omega, 0}\right)^{1 / 2}\left(1+\delta_{\Omega^{\prime}, 0}\right)^{1 / 2}} \sum_{l^{\prime}, l} \frac{\left[l, l^{\prime}\right]}{[J]^{2}}\left\langle j^{\prime} \Omega^{\prime}, l^{\prime} 0 \mid J \Omega^{\prime}\right\rangle S_{j^{\prime} l^{\prime}, j l}^{J P}\langle j \Omega, l 0 \mid J \Omega\rangle
$$

where conservation of parity implies that $P=(-1)^{l+j}=(-1)^{l^{\prime}+j^{\prime}}$,

$$
[a, b, \ldots, z]=\sqrt{(2 a+1)(2 b+1) \cdots(2 z+1)}
$$

and some labels of the scattering matrix elements (representing quantities which are not affected by the transformation: total energy, vibrational quantum numbers, arrangement indices) have been omitted for simplicity.

In the ultracold limit, where only the $l=0$ collisions are reactive, the scattering matrix elements in the orbital angular momentum representation fulfill

$$
S_{j^{\prime} l^{\prime}, j l}^{J P}=0 \quad \text { if } \quad l \neq 0
$$


and the transformation $(7.3)$ reads

$$
\begin{aligned}
S_{j^{\prime} \Omega^{\prime}, j \Omega}^{J P} & =\frac{2}{\left(1+\delta_{\Omega, 0}\right)^{1 / 2}\left(1+\delta_{\Omega^{\prime}, 0}\right)^{1 / 2}} \sum_{l^{\prime}} \frac{\left[l^{\prime}\right]}{[J]^{2}}\left\langle j^{\prime} \Omega^{\prime}, l^{\prime} 0 \mid J \Omega^{\prime}\right\rangle S_{j^{\prime} l^{\prime}, j 0}^{J P} \underbrace{\langle j \Omega, 00 \mid J \Omega\rangle}_{\delta_{j J}} \\
& =\frac{2 \delta_{j J}}{\left(1+\delta_{\Omega, 0}\right)^{1 / 2}\left(1+\delta_{\Omega^{\prime}, 0}\right)^{1 / 2}} \sum_{l^{\prime}} \frac{\left[l^{\prime}\right]}{[J]^{2}}\left\langle j^{\prime} \Omega^{\prime}, l^{\prime} 0 \mid J \Omega^{\prime}\right\rangle S_{j^{\prime} l^{\prime}, j 0}^{J P}
\end{aligned}
$$

showing that the only nonvanishing elements of the helicity-representation scattering matrix are those with $J=j$ and that they depend on the reactants helicity $(\Omega)$ through the $\left(1+\delta_{\Omega, 0}\right)^{1 / 2}$ factor.

When $S_{j^{\prime} \Omega^{\prime}, j \Omega}^{j P=+1}$ and $S_{j^{\prime} \Omega^{\prime}, j \Omega}^{j P=-1}$ (given by Eq. (7.6) ) are combined according to Eqs. (3.41) and (3.42) in order to form the elements $S_{j^{\prime} \Omega^{\prime}, j \Omega}^{j}$ that represent the starting point for the intrinsic moments calculations, it is found that the factors containing the dependence on $\Omega$ cancel out and that, therefore,

$$
S_{j^{\prime} \Omega^{\prime}, j \Omega}^{j}=S_{j^{\prime} \Omega^{\prime}, j}^{j}
$$

is independent on the reactants helicity value. The explicit expression for these elements is given by

$$
S_{j^{\prime} \Omega^{\prime}, j}^{j}=\sum_{l^{\prime}} \frac{\left[l^{\prime}\right]}{[j]^{2}}\left\langle j^{\prime} \Omega^{\prime}, l^{\prime} 0 \mid j \Omega^{\prime}\right\rangle\left[S_{j^{\prime} l^{\prime}, j 0}^{j P=+1}+S_{j^{\prime} l^{\prime}, j 0}^{j P=-1}\right]
$$

where, depending on the value of $j$, only one of these terms appears

$$
\begin{aligned}
\text { If } j=\text { even } & \rightarrow j^{\prime}+l^{\prime} \text { has to be even }(P=+1) \\
\text { If } j=\text { odd } & \rightarrow j^{\prime}+l^{\prime} \text { has to be odd }(P=-1)
\end{aligned}
$$

\subsubsection{PPs and PDDCSs for ultracold reactions}

The state-to-state PDDCSs can be worked out from the parity adapted elements of the scattering matrix in the helicity representation as follows (Eq. (4.17))

$$
\left[S_{q}^{(k)}(\theta)\right]^{*}=\sum_{\Omega^{\prime} \Omega_{1} \Omega_{2}} \bar{f}_{\Omega^{\prime} \Omega_{1}}(\theta) \bar{f}_{\Omega^{\prime} \Omega_{2}}^{*}(\theta)\left\langle j \Omega_{1}, k q \mid j \Omega_{2}\right\rangle
$$

where the scattering amplitudes $\bar{f}_{\Omega^{\prime} \Omega}(\theta)$ are given by Eq. (4.18)

$$
\bar{f}_{\Omega^{\prime} \Omega}(\theta)=\frac{\sum_{J}(2 J+1) d_{\Omega^{\prime} \Omega}^{J}(\theta) S_{j^{\prime} \Omega^{\prime}, j \Omega}^{J}}{N}
$$


and the factor $N$ is

$$
N=\left[2 \sum_{J \Omega^{\prime} \Omega}(2 J+1)\left|S_{j^{\prime} \Omega^{\prime}, j \Omega}^{J}\right|^{2}\right]^{1 / 2}
$$

Let us assume that we are in the ultracold limit and only $l=0$ collisions are reactive. The first consequence of this assumption is that only the $J=j$ partial wave has to be taken into account and that Eq. (7.11) can be written as

$$
\left[S_{q}^{(k)}(\theta)\right]^{*}=\sum_{\Omega^{\prime} \Omega_{1} \Omega_{2}} A d_{\Omega^{\prime} \Omega 1}^{j}(\theta) S_{j^{\prime} \Omega^{\prime}, j \Omega_{1}}^{j} d_{\Omega^{\prime} \Omega_{2}}^{j}(\theta) S_{j^{\prime} \Omega^{\prime}, j \Omega_{2}}^{j *}\left\langle j \Omega_{1}, k q \mid j \Omega_{2}\right\rangle
$$

where $J$ has been substituted by $j$ and A holds for

$$
A=\frac{[j]^{4}}{N^{2}}
$$

with $\mathrm{N}$ given by

$$
N=\left[2(2 j+1)^{2}\left(\left|S_{j^{\prime} 0, j}^{j}\right|^{2}+2 \sum_{\Omega^{\prime}>0}\left|S_{j^{\prime} \Omega^{\prime}, j}^{j}\right|^{2}\right)\right]^{1 / 2}
$$

Expression (7.14) can be further simplified because the scattering matrix elements do no depend on $\Omega$

$$
S_{q}^{(k)}(\theta)=A \sum_{\Omega^{\prime}}\left|S_{j^{\prime} \Omega^{\prime}, j}^{j}\right|^{2} \sum_{\Omega_{1} \Omega_{2}} d_{\Omega^{\prime} \Omega_{1}}^{j}(\theta) d_{\Omega^{\prime} \Omega_{2}}^{j}(\theta)\left\langle j \Omega_{1}, k q j \Omega_{2}\right\rangle
$$

The asterisk has been suppressed because the PDDCSs are obviously real (all the quantities involved in the different sums are real).

The following relationship between reduced matrix elements and Clebsch-Gordan coefficients

$$
\sum_{N_{1} N_{2}} d_{M_{1} N_{1}}^{J_{1}}(\theta) d_{M_{2} N_{2}}^{J_{2}}(\theta)\left\langle J_{1} N_{1} J^{\prime} N^{\prime} \mid J_{2} N_{2}\right\rangle=\sum_{M}\left\langle J_{1} M_{1} J^{\prime} M \mid J_{2} M_{2}\right\rangle d_{M N^{\prime}}^{J^{\prime}}(\theta)
$$

is proved in Appendix El Setting $J_{1}=J_{2}=j, N_{1}=\Omega_{1}, J^{\prime}=k, M_{1}=M_{2}=\Omega^{\prime}$, $N_{2}=\Omega_{2}$ and $N^{\prime}=q$, the left hand term of Eq. (7.18) coincides with the double sum to the right of Eq. (7.17), which can be rewritten as

$$
S_{q}^{(k)}(\theta)=A d_{0 q}^{k}(\theta) \sum_{\Omega^{\prime}}\left\langle j \Omega^{\prime}, k 0 \mid j \Omega^{\prime}\right\rangle\left|S_{j^{\prime} \Omega^{\prime}, j}^{j}\right|^{2}
$$

Expanding this sum and employing the symmetries of the Clebsch-Gordan coefficients 
[37] and the scattering matrix (Eq. (3.43)), we obtain the following expression for the state-to-state PDDCSs in the ultracold limit

$$
\begin{aligned}
S_{q}^{(k)}(\theta)= & \frac{[j]^{4}}{N^{2}} d_{0 q}^{k}(\theta)\left\{\left|S_{j^{\prime} 0, j}^{j}\right|^{2}\langle j 0, k 0 \mid j 0\rangle+\right. \\
& {\left.\left[1+(-1)^{k}\right] \sum_{\Omega^{\prime}>0}\left(\left|S_{j^{\prime} \Omega^{\prime}, j}^{j}\right|^{2}\left\langle j \Omega^{\prime}, k 0 \mid j \Omega^{\prime}\right\rangle\right)\right\} }
\end{aligned}
$$

A detailed analysis of this Equation shows that

- The $\mathrm{k}=0$ moment is independent on the scattering angle: $S_{0}^{(0)}(\theta)=1 / 2$. This is the mathematical expression for the fact that, in the absence of reactant polarisation, $\ell=0$ differential cross sections are isotropic. This statement can be proved from Eq. (7.20) by employing Eq. (7.16), $d_{00}^{0}(\theta)=1$ [37. and $\left\langle j \Omega^{\prime}, 00 \mid j \Omega^{\prime}\right\rangle=1[37$.

- All the PDDCSs with $k$ odd vanish ${ }^{2}$.

Integration of the PDDCSs over the scattering angle provides us with the polarisation parameters (Eq. (4.11)). The k odd polarisation parameters in the ultracold limit must be necessarily zero because the corresponding PDDCSs are zero as well. Regarding the $\mathrm{k}$ even polarisation parameters, their evaluation will involve the following integral

$$
\begin{aligned}
s_{q}^{k} & \propto \int_{0}^{\pi} d_{0 q}^{k}(\theta) \sin \theta d \theta \\
& \stackrel{38}{=}(-1)^{q}\left[\frac{(k-q) !}{(k+q) !}\right]^{1 / 2} \int_{-1}^{1} P_{k q}(\cos \theta) d(\cos \theta)
\end{aligned}
$$

where $P_{k q}(\cos (\theta))$ represents an associated Legendre polynomial. These polynomials display the following properties 150 .

$$
\int_{-1}^{1} P_{k 0}(\cos \theta) d(\cos \theta) \propto \delta_{k, 0}
$$

and

$$
\begin{aligned}
& P_{k q}(\cos \theta)=-P_{k q}(-\cos \theta) \text { if } k+q \text { is odd } \\
& P_{k q}(\cos \theta)=P_{k q}(-\cos \theta) \text { if } k+q \text { is even }
\end{aligned}
$$

that, when combined with Eq. (7.21), allow for stating that, in the $E_{\text {coll }} \rightarrow 0$ limit,

\footnotetext{
${ }^{2}$ The Clebsch-Gordan coefficient $\langle j 0, k 0 \mid j 0\rangle$ is zero for $k$ odd 37
} 
only the polarisation parameters $s_{0}^{0}$ and $s_{q}^{k}$ even (with $q \neq 0$ ) can be different from zero.

It is convenient to stress that, while the $k$ odd polarisation parameters vanish because the corresponding PDDCS are zero, the $s_{0}^{k \text { even }}(k \neq 0)$ and $s_{q}^{k \text { oven }}$ odd polarisation parameters cancel out because, although the corresponding PDDCSs can be different from zero, their symmetry is such that the integral over the scattering angle vanishes.

\subsubsection{Reaction control in the ultracold limit}

As shown in Chapter 4, stereodynamical control of integral cross section is only possible in the presence of nonvanishing intrinsic PPs with $k>0$ and $q=0$ (Eq. (4.23)). This implies that, when only the $l=0$ partial wave contributes to reactivity, selection of reactants polarisation cannot have any effect on the reaction ICS.

On the other hand, and as also shown in Chapter 4, all that stereodynamical control of the product recoil direction requires is nonvanishing intrinsic PPs with $k>0$ (for the special integral cross section, Eq. (4.25)) or nonvanishing intrinsic PDDCSs with $k>0$ (for the differential cross section, Eq. (4.20) ). As in the $l=0$ case PDDCSs (PPs) with $k$ even (with both $k$ and $q$ even) can, and in general do, have nonzero values, selection of reactants polarisation does influence the reaction differential cross section (special integral cross section).

\section{3. $\mathbf{F}+\mathrm{H}_{2}$ reaction stereodynamics at low and ultralow collision energies}

We illustrate the results presented in the former Section by analysing the

$$
\mathrm{F}+\mathrm{H}_{2}(v=0, j=2) \rightarrow \mathrm{HF}\left(v^{\prime}, j^{\prime}\right)+\mathrm{H}
$$

reaction mechanism and control in the cold and ultracold regime.

The $\mathrm{F}+\mathrm{H}_{2}$ ultracold reactive collisions [147] and its isotopic variants [151, 152] have been already studied in order to determine the effect of reactants excitation with one quantum of vibration or two quanta of rotation on the reaction. Surprisingly ${ }^{3}$, reactant vibrational excitation was found to decrease the reaction cross section by as much as four orders of magnitude [147. The consequences of rotational excitation

\footnotetext{
${ }^{3}$ When the collision energy is very low, even a mundane reaction barrier is, in relative terms, very high. Under such conditions, internal excitation of reactants may provide enough energy for the reactants to overcome, or at least facilitate their tunnelling through, the reaction barrier. Therefore, one could reasonably expect a reactivity increase as the reactants get internally excited
} 
were less clear-cut. While reaction cross sections changed little [147, de-excitation cross sections were found to be sensitive to isotopic composition; for the $\mathrm{F}+\mathrm{D}_{2}$ reaction, rotational quenching was predicted to be more efficient than reaction 147, 151 .

\subsubsection{Reactive scattering calculations}

Calculation of the intrinsic moments requires, as input data, scattering matrices in the helicity representation. The ones used here were obtained with the ABC code [75] and the Stark-Werner potential energy surface 125. The collision energies considered were in the $1 \mu \mathrm{eV}-43 \mathrm{meV}$ range.

Some of the ABC execution parameters were the same for all calculations: the cutoff energy for the rovibrational basis sets was fixed at $2.5 \mathrm{eV}$, and the maximum rotational angular momentum and helicity quantum numbers at $j_{\max }=22$ and $\Omega_{\max }=7$.

Other parameters had to be changed according to the energy. The maximum hyperradius and integration step ranged from $\rho_{\max }=200 a_{0}$ ( $a_{0}$ represents the Borh radius) and $\Delta \rho=0.005 a_{0}$ for the calculations at $E_{\text {coll }}=1 \mu \mathrm{eV}$ to $\rho_{\max }=15 a_{0}$ and $\Delta \rho=0.08 a_{0}$ for the calculations at $E_{\text {coll }}=43 \mathrm{meV}$.

The suitability of the execution parameters was verified by analysing the total reaction probabilities and product rotational populations obtained with different parameter sets and for zero total angular momentum. The parameters used to generate the final results were such as to ensure full convergence of the calculation.

\subsubsection{Results}

We start the presentation of our results with Fig. 7.1, which shows the collision energy dependence of the integral cross section (ICS) of reaction (7.25) summed over product states and in the absence of reactant polarisation; note that both axes of the figure use a logarithmic scale.

The ICS features a minimum at $E_{\text {coll }} \approx 5 \mathrm{meV}$ and increases steadily with decreasing collision energy; at $E_{\text {coll }} \approx 5 \mu \mathrm{eV}$, it is larger than at the relatively high energies (the three highest of Fig. 7.1) used in the 1985 measurements by the Yuan Lee group [120. In particular, the lowest-energy reaction cross section we have calculated $\left(E_{\text {coll }}=1 \mu \mathrm{eV}\right)$ is about three times as large as the ones obtained in those experiments.

The remarkably high $\mathrm{F}+\mathrm{H}_{2}$ reactivity at ultralow energies was first noted by Balakrishnan and Dalgarno 149, and further analysed by Bodo and coworkers 145 , 


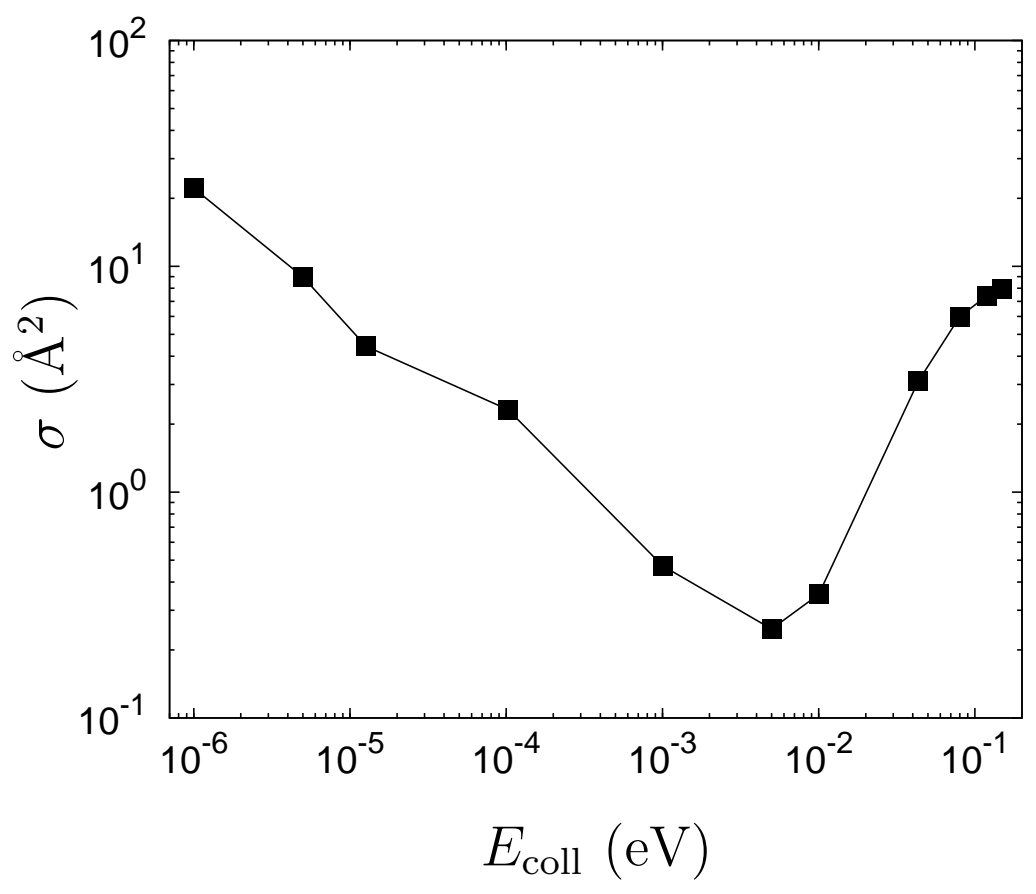

Fig. 7.1.: Collision energy dependence of the integral cross section of the $\mathrm{F}+\mathrm{H}_{2}(v=$ $0, j=2) \rightarrow \mathrm{HF}\left(v^{\prime}, j^{\prime}\right)+\mathrm{H}$ reaction summed over product states and in the absence of reactant polarisation. The points are the calculated results; the line simply joins them. The three largest energies correspond to those of Lee and co-workers experiment (see Chapter 6).

147, who attributed it to coupling to a virtual state associated with the van der Waals well in the reactants valley.

We also note that, to the left of the $E_{\text {coll }} \approx 5 \mathrm{meV}$ minimum, the slope of the ICS is approximately $-1 / 2$. This seems to indicate that the Wigner limit [148, where the reaction cross section satisfies Eq. (7.1) and reactivity is due to zero orbital angular momentum collisions, is reached very quickly. This, however, is not strictly correct, as evidenced by the plots of the total reaction probability as a function of the total angular momentum quantum number included in Fig. 7.2 , the plots shown are for collision energies in the $1-100 \mu \mathrm{eV}$ range and for unpolarised reactants.

Since the reaction under consideration here involves the $\mathrm{H}_{2}$ molecule in a $j=2$ state, the $l=0$ condition implies $J=2$. As the results in Fig. 7.2 demonstrate, only at the very lowest collision energies, $E_{\text {coll }} \lesssim 5 \mu \mathrm{eV}$, does reactivity become due to the $J=2$ partial wave only. For higher collision energies, convergence of the calculated 


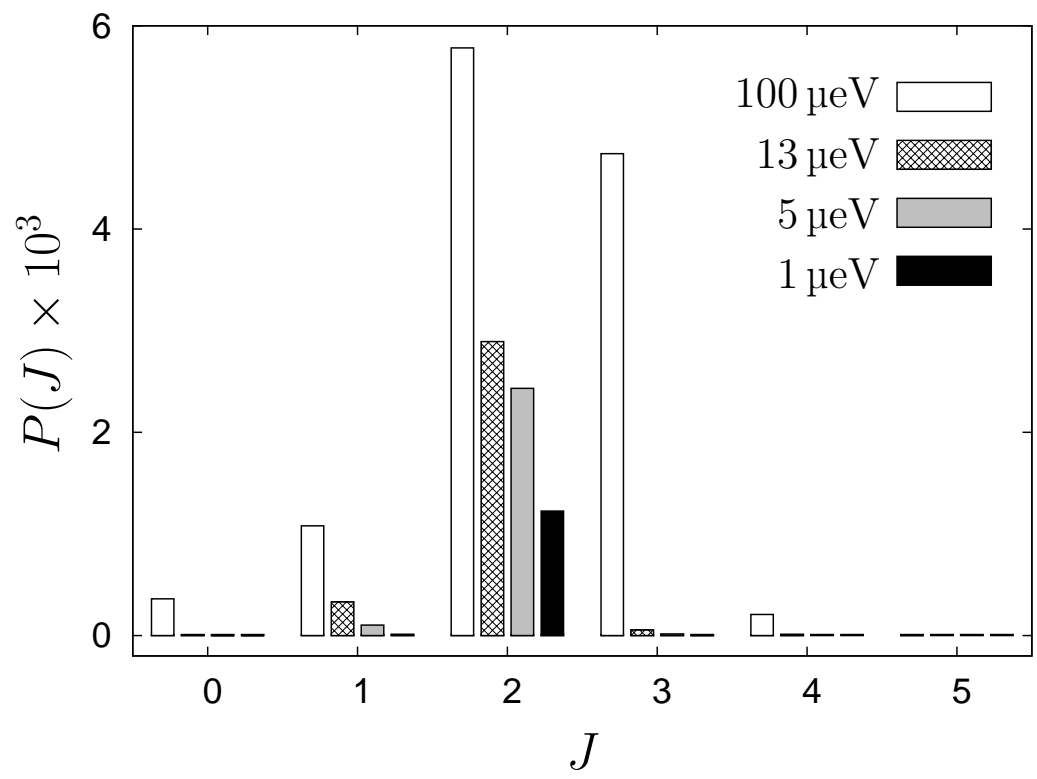

Fig. 7.2.: Reaction probability as a function of the total angular momentum quantum number for the $\mathrm{F}+\mathrm{H}_{2}(v=0, j=2) \rightarrow \mathrm{HF}\left(v^{\prime}=\right.$ all, $j^{\prime}=$ all $)+\mathrm{H}$ reaction at collision energies between 1 and $100 \mu \mathrm{eV}$ and in the absence of reactant polarisation.

cross sections requires consideration of other partial waves.

Other interesting aspects of the dependence of the $\mathrm{F}+\mathrm{H}_{2}(v=0, j=2)$ reactivity on the reactants orbital angular momentum are revealed by the vibrationally-resolved probability functions included in Fig. 7.3, the results shown are for $v^{\prime}=2$ (top panel) or $v^{\prime}=3$ (bottom panel).

Comparison between the two panels of Fig. 7.3 shows that at $E_{\text {coll }}=100 \mu \mathrm{eV}$ (when the reaction is clearly above the Wigner threshold) orientation of the reactants orbital angular momentum correlates with the product vibrational state. Reactions forming $\operatorname{HF}\left(v^{\prime}=2\right)$ are favoured by $J \geq 2$ and, therefore, $\ell \|+\mathbf{j}$. In contrast, reactions forming $\operatorname{HF}\left(v^{\prime}=3\right)$ are favoured by $J \leq 2$ and, therefore, $\boldsymbol{\ell} \|-\mathbf{j}$. This effect, which is clearly visible here, provides further evidence of the $\mathbf{j}$ orientation importance for chemical reactions.

The reaction probability functions at $E_{\text {coll }}=5 \mu \mathrm{eV}$ deserve an additional comment. When they are vibrationally resolved, a small $J=1$ contribution is found to remain and to be most relevant for reactions into the $v^{\prime}=3$ products state. This indicates that the rate at which the reaction approaches the Wigner threshold depends not only on the collision energy, but also on the product state. 

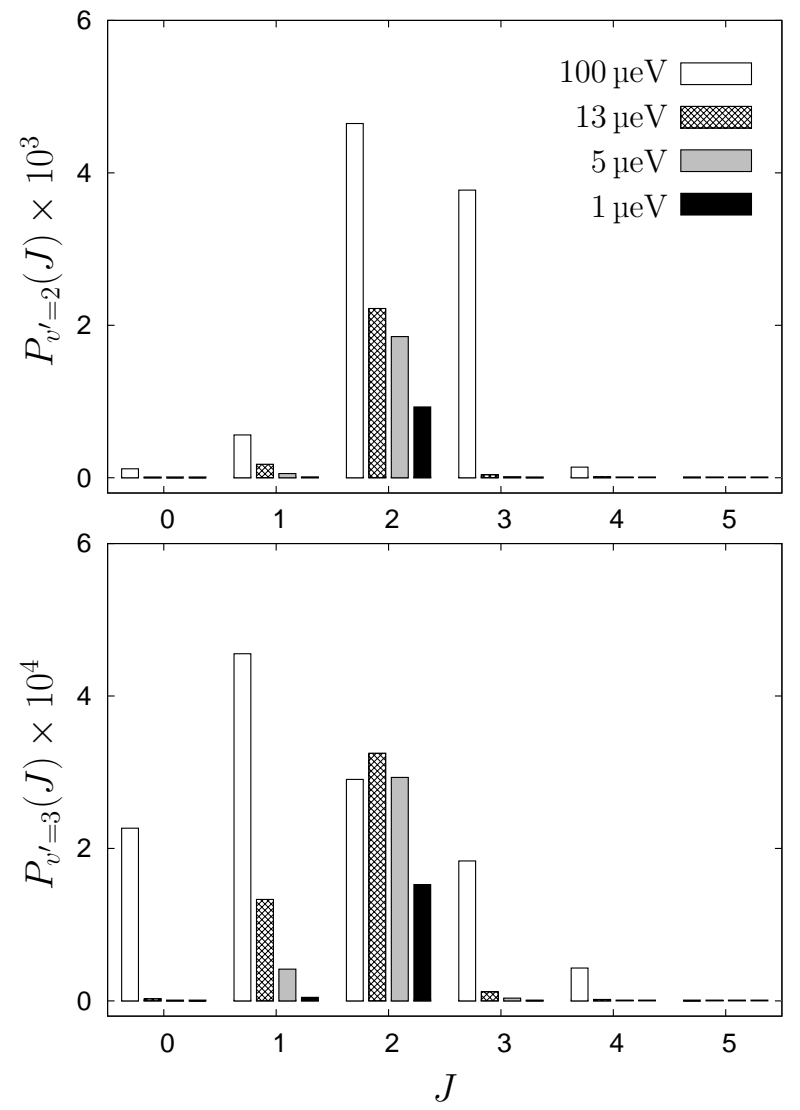

Fig. 7.3.: Reaction probability as a function of the total angular momentum quantum number for the $\mathrm{F}+\mathrm{H}_{2}(v=0, j=2) \rightarrow \mathrm{HF}\left(v^{\prime}, j^{\prime}=\right.$ all $)+\mathrm{H}$ reaction at collision energies between 1 and $100 \mu \mathrm{eV}$, for $v^{\prime}=2$ (top) or $v^{\prime}=3$ (bottom) and in the absence of reactant polarisation.

We now turn to state-to-state results, starting with those in Fig. 7.4 product rotational state distributions associated with reaction into $\mathrm{HF}\left(v^{\prime}=2\right)$ at $E_{\text {coll }}=$ $100 \mu \mathrm{eV}$ and various reactant polarisations. The intrinsic portraits representing the $\mathrm{H}_{2}(v=0, j=2)$ polarisation leading to products formation into selected rotational states are shown on the top of Fig. 7.4. Their extrinsic counterparts (those associated with the various $\beta$ values) are shown on Fig. 4.4.

The results on Fig. 7.4 provide a first illustration of the reaction control possibilities at collision energies that, while low, are above those at which the reaction follows the Wigner threshold laws.

In this case, it is clear that appropriate selection of reactant polarisation can have 

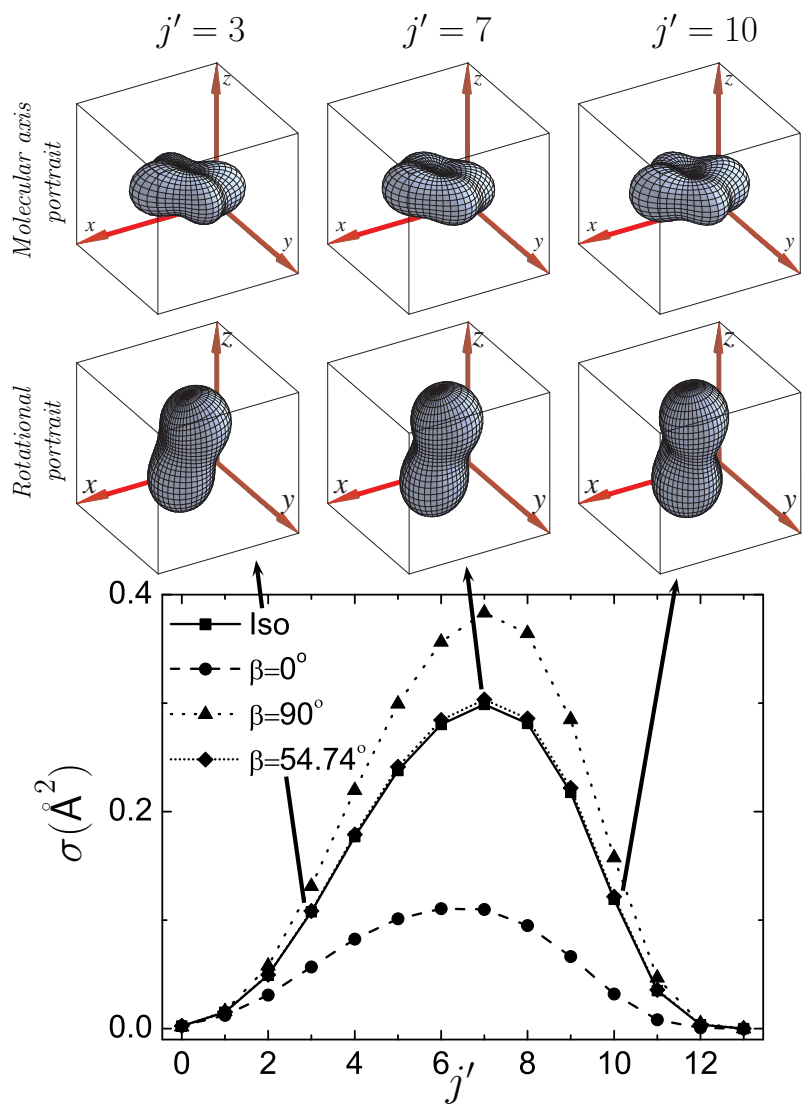

Fig. 7.4.: Product rotational state distributions associated with the $\mathrm{F}+\mathrm{H}_{2}(v=0, j=$ $2) \rightarrow \operatorname{HF}\left(v^{\prime}=2, j^{\prime}\right)+\mathrm{H}$ reaction at $E_{\text {coll }}=100 \mu \mathrm{eV}$ and various reactant polarisations. The stereodynamical portraits on the top show the intrinsic $\mathrm{H}_{2}(v=0, j=2)$ polarisations for selected product rotational states.

a large impact on the reaction cross section. Side-on, $\beta=90^{\circ}$, collisions considerably enhance reactivity, whereas head-on, $\beta=0^{\circ}$ collisions largely suppress it. Comparison between the intrinsic and extrinsic stereodynamical portraits shows why: preparation of $\mathrm{H}_{2}$ molecules with $\beta=90^{\circ}$ polarisation gives to the reaction something quite close to what the reaction "wants," whereas preparation of $\mathrm{H}_{2}$ molecules with $\beta=0^{\circ}$ polarisation does the opposite.

Consideration of the intrinsic stereodynamical portraits on Fig. 7.4 also shows why the selection of different $\beta$ values for the $\mathrm{H}_{2}$ polarisation allows for considerable control of the total reactivity, but not of the product state distribution. The intrinsic stereodynamical portraits hardly differ. In other terms, the reaction's steric requirements 


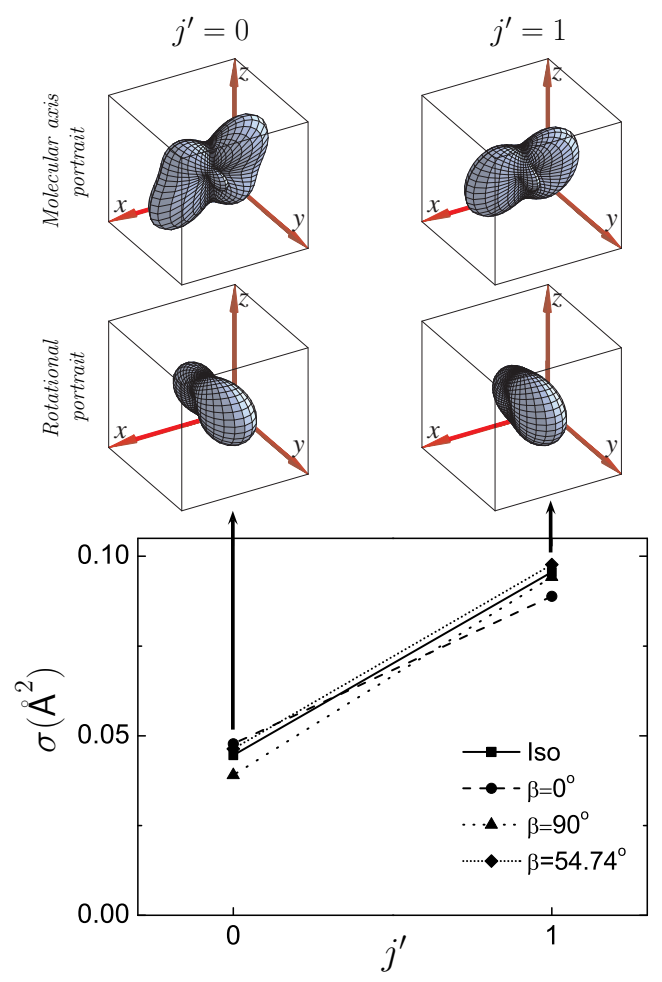

Fig. 7.5.: Product rotational state distributions associated with the $\mathrm{F}+\mathrm{H}_{2}(v=0, j=$ $2) \rightarrow \operatorname{HF}\left(v^{\prime}=3, j^{\prime}\right)+\mathrm{H}$ reaction at $E_{\text {coll }}=100 \mu \mathrm{eV}$ and various reactant polarisations. The stereodynamical portraits on the top show the intrinsic $\mathrm{H}_{2}(v=0, j=2)$ polarisations for the two opened rotational states.

are largely independent of the product rotational state.

Intrinsic $\mathrm{H}_{2}(v=0, j=2)$ stereodynamical portraits and product rotational state distributions for reaction into $\operatorname{HF}\left(v^{\prime}=3, j^{\prime}\right)$ at the same collision energy and with the same extrinsic reactant polarisations are shown on Fig. 7.5.

The stereodynamical portraits on Fig. 7.5 provide striking illustrations of the distinctiveness of the mechanism of reactions into $v^{\prime}=3$ products: they reveal steric requirements that are very different from those obtained for $v^{\prime}=2$ (the portraits obtained for $v^{\prime}<2$, not shown here, are very similar to the ones for $v^{\prime}=2$ ). Reactivity into $v^{\prime}=3$ requires the $\mathrm{H}_{2}$ reactant to have its interatomic axis on the scattering plane, and can involve rotational orientation effects. Note, however, that the extent to which reactivity can be controlled is rather limited. This is so because manipulation of the reactivity would require selection of values for both $\beta$ and $\alpha$. Reactant polarisation along $\beta=90^{\circ}, \alpha=0^{\circ}$ would increase the reactivity, while reactant po- 


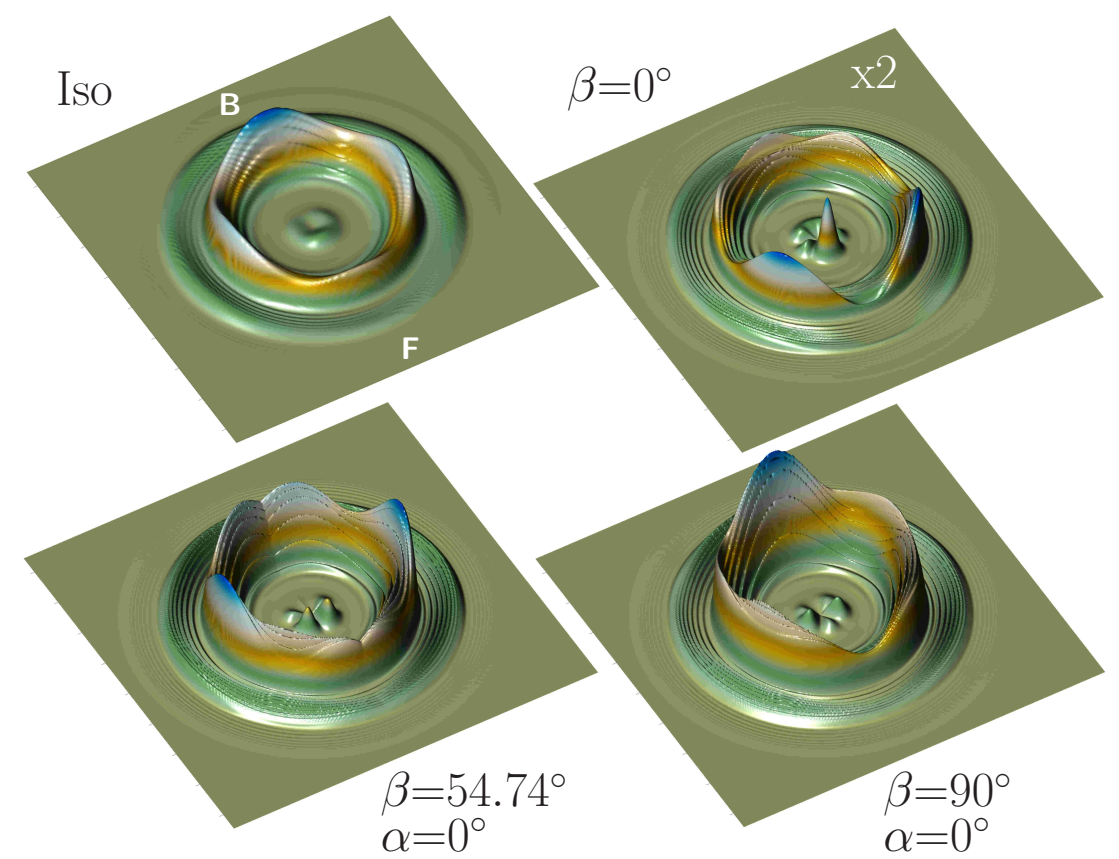

Fig. 7.6.: Quantum triple (angle-velocity) differential cross section (polar maps) of the $\mathrm{F}+\mathrm{H}_{2}(v=0, j=2)$ reaction at $E_{\text {coll }}=100 \mu \mathrm{eV}$ for selected extrinsic reactant polarisations. "F" and "B" denote forward and backward scattering scattering angles, $\theta=0^{\circ}$ and $180^{\circ}$. The figure for $\beta=0^{\circ}$ has been multiplied by two.

larisation along $\beta=\alpha=90^{\circ}$ would decrease it. Selection of $\alpha$ values, however, is not possible when the property being manipulated is the integral cross section (see Sec. 4.6).

Differential cross sections (DCSs) also calculated at $E_{\text {coll }}=100 \mu \mathrm{eV}$ and for selected extrinsic reactant polarisations are presented on Fig. 7.6. It provides further illustration of the sensitivity of the reaction to reactant polarisation and of the distinctive dynamics of reactions into $\operatorname{HF}\left(v^{\prime}=3\right)$ products.

In the absence of reactant polarisation ("Iso" polar map), $v^{\prime}=2$ products (most intense set of rings in all panels) are scattered mostly in backward and sideways directions, while $v^{\prime}=3$ products (innermost rings in all panels) recoil along sideways and forward directions.

The effect of head-on collisions $\left(\beta=0^{\circ}\right.$ reactant polarisation) is to depress the $v^{\prime}=2$ DCS at all scattering directions, and to sharply focus the $v^{\prime}=3$ DCS around $\theta=0^{\circ}$. 
Coplanar, magic angle collisions $\beta=54.74^{\circ}, \alpha=0^{\circ}$ have a very different effect: reactivity into $\operatorname{HF}\left(v^{\prime}=2\right)$ is shifted to different scattering angles (sideways scattering becomes dominant and forward scattering gets enhanced). And so is reactivity into $\operatorname{HF}\left(v^{\prime}=3\right)$, albeit in a different way. The $v^{\prime}=3$ DCS is again sharply focused at forward scattering angles, but not at $\theta=0^{\circ}$. Instead, reactivity into $v^{\prime}=3$ and $\theta=0^{\circ}$ is completely suppressed.

Coplanar, side-on collisions $\beta=90^{\circ}, \alpha=0^{\circ}$ influence the reaction in yet another way. The $v^{\prime}=2$ DCS is magnified without being overly distorted, whereas the $v^{\prime}=3$ DCS is again focused, but this time around $\theta=0^{\circ}$ as well as around other, close-tosideways scattering angles.

In short, the integral and differential cross sections presented above show that at low collision energies the $\mathrm{F}+\mathrm{H}_{2}(v=0, j=2)$ reaction has a very sensitive stereodynamics, and is largely amenable to external control.

We now turn to the ultracold regime, under which the reaction mostly follows the Wigner laws. From this point on, our results will refer to reactions at $E_{\text {coll }}=1 \mu \mathrm{eV}$.

Product rotational state distributions associated with reactions into $\operatorname{HF}\left(v^{\prime}=2\right)$ and various reactant polarisations are shown on Fig. 7.7. As the figure clearly indicates, and despite the clear anisotropy of the intrinsic stereodynamical portraits, in the ultracold regime one cannot control integral cross sections by selecting extrinsic reactant polarisations.

Intrinsic $\mathrm{H}_{2}(v=0, j=2)$ stereodynamical portraits and product rotational state distributions for reaction into $\mathrm{HF}\left(v^{\prime}=3, j^{\prime}\right)$ at the same collision energy and with the same extrinsic reactant polarisations are shown on Fig. 17.8, Although the intrinsic stereodynamical portraits are even more anisotropic than the ones obtained in the $v^{\prime}=2$ case, the end result is the same: stereodynamical control of the reaction ICS is not possible.

It is also interesting to note that although reactions into both $\operatorname{HF}\left(v^{\prime}=2\right)$ and $\mathrm{HF}\left(v^{\prime}=3\right)$ display an intrinsic preference for a certain rotational orientation of the $\mathrm{H}_{2}$ reactant, the preferred sense of $\mathrm{H}_{2}$ rotation gets inverted in going from $v^{\prime}=2$ to $v^{\prime}=3$. The former shows a preference for what, as viewed from the $y>0$ side of the scattering plane, is clockwise $\mathrm{H}_{2}$ rotation; the latter for anticlockwise $\mathrm{H}_{2}$ rotation.

Having considered a reaction property (the ICS) that under ultracold conditions is not amenable to stereodynamical control, we now turn to a property that, even at the lowest of collision energies, still depends on the collision geometry: the product angular distribution. Differential cross sections (DCSs) calculated at $E_{\text {coll }}=1 \mathrm{\mu eV}$ and for selected extrinsic reactant polarisations are presented on Fig. 7.9.

The DCSs of Fig. 7.9 feature three remarkable characteristics: they have very varied 


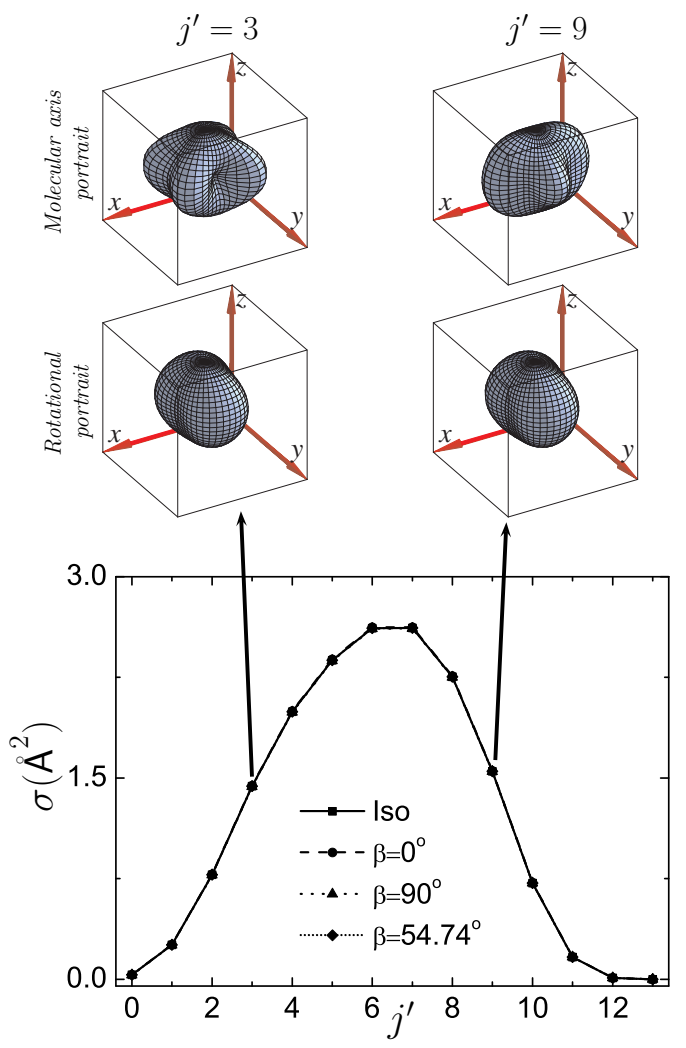

Fig. 7.7.: Product rotational state distributions associated with the $\mathrm{F}+\mathrm{H}_{2}(v=0, j=$ $2) \rightarrow \mathrm{HF}\left(v^{\prime}=2, j^{\prime}\right)+\mathrm{H}$ reaction at $E_{\text {coll }}=1 \mu \mathrm{eV}$ and various reactant polarisations. The curves obtained with the different polarisation conditions are practically indistinguishable. The stereodynamical portraits on the top show the intrinsic $\mathrm{H}_{2}(v=0, j=2)$ polarisations for selected product rotational states.

shapes, all are highly symmetrical, and the angular distributions of the $v^{\prime}=2$ and $v^{\prime}=3$ products can be independently controlled.

In the absence of extrinsic reactant polarisation, the reaction DCS is just what one would expect for $\ell=0$ collisions: it has the perfectly isotropic shape of Yorkshire pudding.

In the presence of extrinsic reactant polarisation, the DCS changes. The resulting shapes, while far from isotropic, are highly symmetric. This is a consequence of the fact that, in the ultracold regime, stereodynamical control of the product angular distribution is due not only to dynamics, but also to pure geometry.

From a chemical point of view, the most interesting observation is that the recoil 


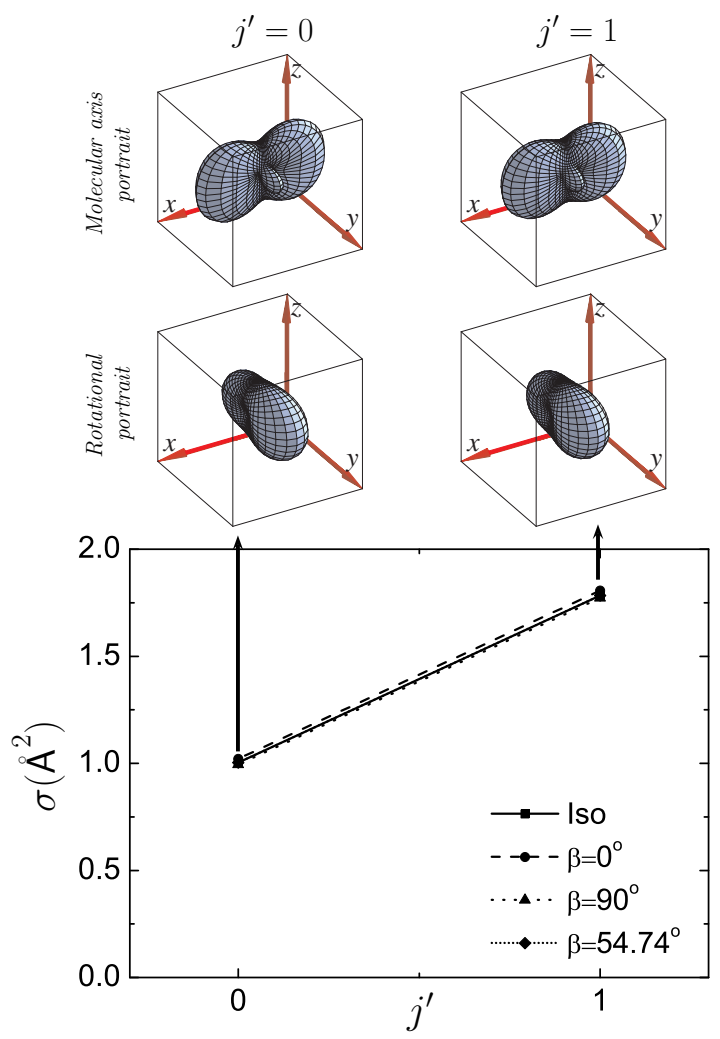

Fig. 7.8.: Product rotational state distributions associated with the $\mathrm{F}+\mathrm{H}_{2}(v=0, j=$ $2) \rightarrow \mathrm{HF}\left(v^{\prime}=3, j^{\prime}\right)+\mathrm{H}$ reaction at $E_{\text {coll }}=1 \mu \mathrm{eV}$ and various reactant polarisations. The curves obtained with the different polarisation conditions are practically indistinguishable. The stereodynamical portraits on the top show the intrinsic $\mathrm{H}_{2}(v=0, j=2)$ polarisations for the two opened rotational states.

directions of $\operatorname{HF}\left(v^{\prime}=2\right)$ and $\operatorname{HF}\left(v^{\prime}=3\right)$ products can be independently manipulated.

If the extrinsic reactant polarisation is the $\beta=0^{\circ}$ one leading to head-on collisions, $v^{\prime}=3$ products are mostly scattered into $\theta=0$ or $180^{\circ}$, while $v^{\prime}=2$ products are mostly scattered into $\theta=45$ or $135^{\circ}$ (note, however, that there is also some probability of $\operatorname{HF}\left(v^{\prime}=3\right)$ scattering into $\theta=90^{\circ}$, and that the angular distribution of high $\operatorname{HF}\left(v^{\prime}=2\right)$ rotational states follows a pattern that is the opposite of the one followed by the dominant, lower $\operatorname{HF}\left(v^{\prime}=2\right)$ rotational states $)$.

If the extrinsic reactant polarisation is the $\beta=90^{\circ}, \alpha=0^{\circ}$ one leading to side-on collisions, the $\operatorname{HF}\left(v^{\prime}=3\right)$ angular distribution gets "inverted" (it peaks at $\theta=90^{\circ}$ rather than at $\theta=0$ and $\left.180^{\circ}\right)$, whereas the $\operatorname{HF}\left(v^{\prime}=2\right)$ angular distribution remains 


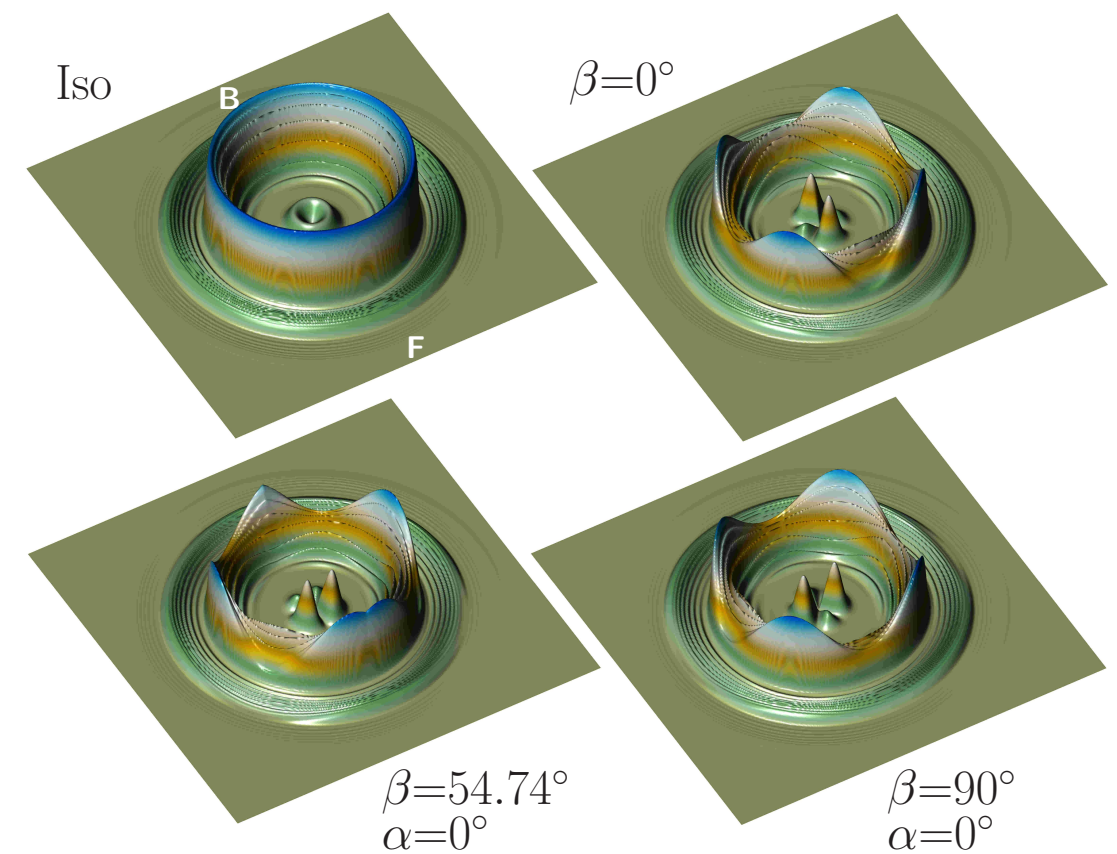

Fig. 7.9.: Quantum triple (angle-velocity) differential cross section (polar maps) of the $\mathrm{F}+\mathrm{H}_{2}(v=0, j=2)$ reaction at $E_{\text {coll }}=1 \mu \mathrm{eV}$ for selected extrinsic reactant polarisations. "F" and "B" denote forward and backward scattering scattering angles, $\theta=0^{\circ}$ and $180^{\circ}$.

largely unchanged (this statement is true for low $\operatorname{HF}\left(v^{\prime}=2\right)$ rotational states; high $\operatorname{HF}\left(v^{\prime}=2\right)$ rotational states follow, as in the previous case, the $\operatorname{HF}\left(v^{\prime}=3\right)$ pattern $)$.

If the collision geometry changes from side-on to one involving "tilted" $\mathrm{H}_{2}$ molecules (this is the case for $\beta=54.74^{\circ}, \alpha=0^{\circ}$ collisions), then the opposite happens: the angular distribution of the $\operatorname{HF}\left(v^{\prime}=2\right)$ products gets inverted, whereas the $\operatorname{HF}\left(v^{\prime}=\right.$ 3 ) angular distribution hardly budges at all (curiously, here the high $\operatorname{HF}\left(v^{\prime}=2\right.$ ) rotational states, while retaining a differentiated angular distribution, do change in much the same way as their low- $j^{\prime}$ counterparts. They have their DCS rings rotated by $\left.45^{\circ}\right)$. 


\section{Conclusions}

- Motivated by the possibility of contributing to an increased understanding of molecular collisions and to a better assessment of experimental control possibilities, it has been presented a theoretical method and proposed a currently feasible experiment for the study of the effect of reactants polarization on the dynamics of atom-diatom collisions.

- The theoretical formalism was stated in general terms, allowing to deal with arbitrary reactant polarization schemes, and to be used in conjunction with both quantum reactive scattering and quasiclassical trajectory calculations. Two aspects of the theory deserve to be pointed out:

- The separation of intrinsic reactant polarizations from their extrinsic counterparts. This splitting makes possible to analyze the collision stereodynamics per se, without consideration of external factors that, while indispensable in practical situations, can be restrictive with regard to analyses of reaction mechanisms. That does not imply, however, that extrinsic polarizations and practical situations cannot be considered: they can, and have been, in a straightforward and flexible way.

- The whole directional information is contained in the scattering matrix, from which it is extracted as a set of intrinsic polarisation moments (PPs and PDDCSs). Each one of these moments is related to the alignment or orientation of $\mathbf{j}$ in the scattering frame when the reaction happens and, all together, they completely describe the intrinsic $\mathbf{j}$ polarisation. However, if we want to use these moments for studying the reaction mechanism, it is essential to obtain a global view that simultaneously takes into consideration the contributions of all the moments. This problem is solved using the 'stereodynamical portraits'. By means of them it is possible to reduce the directional information contained in the intrinsic polarisation to "pictures" that allow the interpretation of polarisation in terms of the mechanism. Because of their interpretative power, the portraits have been the basis of our analysis of the mechanism and, without a doubt, they are 
the most important tool employed in this work, representing the materialisation of the "chemical shape of colliding molecules" introduced by Levine [113] in the early 90 's.

- In terms of experimental control possibilities, this work describes an experiment that, besides allowing for stringent tests of theoretical predictions, is feasible with current technology, requiring only certain modifications of already-existing experimental apparatuses.

- Quantum and quasiclassical results for the benchmark $\mathrm{H}+\mathrm{D}_{2}$ reaction were firstly employed to illustrate how the theoretical methods can be used and what can be achieved in the proposed experiment. In so doing, it was found that $\mathrm{D}_{2}$ polarization, and alignment in particular, has (theoretically at least) a large influence on reaction cross sections, products state distributions and products recoiling direction.

- An exhaustive consideration of the $\mathrm{H}+\mathrm{D}_{2}$ reactive collisions allowed for characterisation of two mechanisms that other authors have identified as main ones in the dynamics of this system: the "dominant" direct mechanism and also the minor but very interesting "delayed" mechanism.

The direct collisions (responsible for the backward and sideways scattering) have been found to take place in a way that is mainly influenced by the range of impact parameters. The $\mathbf{r}$ portraits clearly illustrate the participation of impact parameters and indicate a clear tilt of the internuclear axis with respect to the incoming atom-diatom direction so that the adoption of the linear geometry of the transition state is facilitated. The rotation sense of the $\mathrm{D}_{2}$ molecule is either related to the rotational excitation of the products or to the direction in which the products are formed. The connection with the rotational excitation of the products is more relevant for the reaction mechanism and, in fact, it dominates the integral results. On the contrary, the relation between the $\mathrm{D}_{2}$ molecule rotation and the direction in which the products are formed only can be appreciated in the differential results, when each value of the scattering angle is considered separately.

The delayed collisions (responsible for the forward scattering) are characterised by swift oscillations on the intrinsic moments that give rise to sudden changes in the shape of the portraits. They are originated by the different $|\Omega|$ contributions and the interference between different partial waves. Regarding the mechanism, the PDDCSs oscillations can be interpreted as a consequence of the existence 
of nearside and farside collisions that interfere with each other. As one or the other kind of collisions prevail, the directional preferences of the reaction and the shape of the portraits change.

- The same techniques were next applied to the $\mathrm{F}+\mathrm{H}_{2}$ reaction, with the finding that the process is highly sensitive to the $\mathrm{H}_{2}$ molecule polarisation and identifying two main mechanisms as responsible for the products formation. These mechanisms were termed as direct and delayed, in clear correspondence with their $\mathrm{H}+\mathrm{D}_{2}$ counterparts, with whom they share most of the features. The importance of the transition state access on the direct mechanisms was, however, less clear-cut than for the $\mathrm{H}+\mathrm{D}_{2}$ reaction. This difference stems from the different PES topologies; while for the BKMP2 PES the access to the transition state is limited to a few geometries around the linear minimum, the relative flat character of the transition state for the SW PES makes such access easier and less sterically demanding, in such a way that other factors can become more decisive in order to determine the mechanism features.

The analysis of the integer state-to-state results confirmed that collisions leading to $\operatorname{HF}\left(v^{\prime}=3\right)$ molecules take place through a different mechanism than those leading to $\operatorname{HF}\left(v^{\prime}=1\right.$ and 2$)$ and proved the feasibility of controlling the products distribution through $\mathrm{H}_{2}$ polarisation. Important as this control is, it is largely outstripped by the predicted backward-sideways DCS control: suitable internuclear axis preparations on the scattering plane allow for drastically changing the DCS shape.

- It has been demonstrated that stereodynamics can be used to probe and manipulate cold and ultracold reactive collisions. Employing the benchmark $\mathrm{F}+\mathrm{H}_{2}$ reaction as a test case, the results have shown enormous promise in both regards. On the one hand, very fine details of the reaction dynamics were investigated. Several particulars of the correlation between collision geometry, collision energy, reactivity and product formation have been examined, yielding much insight into the reaction mechanism. On the other hand, selection of reactant polarisation proved to be an useful way of controlling the reaction outcome. The reactant polarisations used in the simulations allowed for fine, product state-selective control of reaction differential and (in the cases of reactions involving a few partial waves other than the $\ell=0$ one) integral cross sections.

- The description of the reaction mechanisms presented in this work do not ex- 
haust all the possibilities. On the contrary, it constitutes a reduced sample. In particular, future work will have to cope with the following open questions:

- We only have considered the products regardless of their polarisation. However, we could imagine an experiment where only the products formed with a defined polarisation were detected. Of course, the corresponding description of the mechanism obtained will change, providing us with new details about how the reactions happen. The method presented in this work is suitable to deal with any polarisation of the products and, in the future, it will be applied to analyse the features of the mechanisms related to the products polarisation.

- Understanding of chemical reactions requires appreciation of when, why and how molecules react, but also of when, why and how they do not. The non-reactive (elastic and inelastic) collision mechanisms also can be studied by means of the intrinsic polarisation, thus completing the results obtained for reactive collisions.

- The processes considered are, in general, not pure. To a certain extent, they are statistical averages of completely independent (pure) processes whose results add up incoherently. Disentanglement of the pure processes taking part in a chemical reaction is another of the goals yet to achieve. 


\section{A. Rotations in quantum mechanics}

This Appendix provides with an introduction to the quantum mechanical treatment of rotations. After briefly presenting some key concepts related to the subject, they will be employed to determine the behaviour under rotations of the polarisation moments.

The reference [37] is an unbeatable handbook about angular momentum quantum theory. All the sketched ideas about the Euler angles, the rotation operator and the rotation matrix together with the symmetry statements and the relations used in the derivations are included and broadened there. In order to avoid annoying repetitions, no citations to this book will be performed in the Appendix, assuming that all the non justified statements and steps can be "filled" by means of it.

As it is always the case with angular momentum algebra, one must be careful with the conventions and definitions used. In the present case, those corresponding to the reference [37] have been chosen (for an illuminating discussion about the different conventions and their connection see [48]).

\section{Euler angles}

Let us consider a $\mathbb{R}^{3}$ reference frame named as $x y z$. Any rotation of that frame gives rise to a second one that will be represented as $X Y Z$. It is always possible to parameterize that rotation by means of the Euler angles $(\alpha, \beta, \gamma)$ which define a three steps prescription for the transformation $x y z \rightarrow X Y Z$

1. Rotate through an angle $\gamma(0 \leq \gamma<2 \pi)$ about the $z$ axis.

2. Rotate through an angle $\beta(0 \leq \beta \leq \pi)$ about the $y$ axis.

3. Rotate through an angle $\alpha(0 \leq \alpha<2 \pi)$ about the $z$ axis.

When the rotation has taken place, $\beta(\alpha)$ is the polar (azimuthal) angle of the $Z$ axis in the $x y z$ frame. 


\section{Rotation operator and rotation matrix}

The rotations of a physical system in the $\mathbb{R}^{3}$ space correspond to rotations in the Hilbert space defined by the system states. These rotations are carried out by means of an unitary operator called rotation operator and defined as

$$
\hat{R}(\alpha, \beta, \gamma)=\hat{R}_{z}(\alpha) \hat{R}_{y}(\beta) \hat{R}_{z}(\gamma)=\exp \left(-i \alpha \hat{\jmath}_{z}\right) \exp \left(-i \beta \hat{\jmath}_{y}\right) \exp \left(-i \gamma \hat{\jmath}_{z}\right)
$$

where $\hat{\jmath}$ is the system angular momentum operator and $\hat{\jmath}_{i}(i=x, y, z)$ are the angular momentum projection operators over the $x y z$ axis.

If our system state corresponds to a vector state $|\phi\rangle$, after the rotation it will be described by $\hat{R}|\phi\rangle$. Equally, any observable $\hat{Q}$ transforms under rotation as

$$
\hat{Q} \rightarrow \hat{R} \hat{Q} \hat{R}^{\dagger}
$$

The representation of the rotation operator on the $|j m\rangle$ basis (eigenvectors of the $\hat{\jmath}^{2}$ and $\hat{\jmath}_{z}$ operators) is given by the rotation matrix

$$
D_{m m^{\prime}}^{(j)}(\alpha, \beta, \gamma)=\left\langle j m|\hat{R}(\alpha, \beta, \gamma)| j m^{\prime}\right\rangle=e^{-i m \alpha} d_{m m^{\prime}}^{(j)}(\beta) e^{-i m^{\prime} \gamma}
$$

where $d_{m m^{\prime}}^{(j)}(\beta)$ is the reduced rotation matrix. From the physical point of view, the rotation matrix describes the transformation between $|j m\rangle$ basis corresponding to different frames of reference. To clarify this, let us consider the effect of an arbitrary rotation over the $|j m\rangle$ ket. Making use of the completeness condition for the basis, it can be found that the rotated state will be

$$
\hat{R}(\alpha, \beta, \gamma)|j m\rangle=\sum_{m^{\prime}} D_{m^{\prime} m}^{(j)}(\alpha, \beta, \gamma)\left|j m^{\prime}\right\rangle
$$

It continues being a $\hat{\jmath}^{2}$ eigenvector $\left(\right.$ as $\left.\left[\hat{\jmath}^{2}, \hat{R}\right]=0\right)$ but not of $\hat{\jmath}_{z}\left(\right.$ as $\left.\left[\hat{\jmath}_{z}, \hat{R}\right] \neq 0\right)$. However, it is straightforward to show that it is an eigenvector of the $\hat{\jmath}_{Z}$ operator ${ }^{1}$ (the angular momentum projection over the $Z$ axis of the frame obtained by rotating $x y z$ through $\alpha, \beta, \gamma)$. So, the former equation can be rewritten as

$$
|j m\rangle_{Z}=\hat{R}(\alpha, \beta, \gamma)|j m\rangle_{z}=\sum_{m^{\prime}} D_{m^{\prime} m}^{(j)}(\alpha, \beta, \gamma)\left|j m^{\prime}\right\rangle_{z}
$$

and the $D_{m^{\prime} m}^{(j)}(\alpha, \beta, \gamma)$ numbers turn out to be the elements of the unitary transfor-

\footnotetext{
${ }^{1}$ The $\hat{\jmath}_{Z}$ operator is given by (see Eq. (A.2)) $\hat{\jmath}_{Z}=\hat{R} \hat{\jmath}_{z} \hat{R}^{\dagger}$. Employing the unitary character of the rotation operator it is found that: $\hat{\jmath}_{Z} \hat{R}|j m\rangle=\hat{R} \hat{\jmath}_{z} \hat{R}^{\dagger} \hat{R}|j m\rangle=\hat{R} \hat{\jmath}_{z}|j m\rangle=\hbar m \hat{R}|j m\rangle$
} 
mation matrix between the $|j m\rangle_{z}$ and the $|j m\rangle_{Z}$ basis.

\section{Quantum mechanical polarisation moments behaviour under rotations}

The polarisation operators $\hat{T}(j)_{k q}$ and $\hat{T}(j)_{k Q}$ corresponding to the $x y z$ and $X Y Z$ reference frames previously defined are given by

$$
\hat{T}(j)_{k q}=\sum_{m, m^{\prime}=-j}^{j}(-1)^{j-m}\left\langle j m^{\prime} j-m \mid k q\right\rangle\left|j m^{\prime}\right\rangle_{z z}\langle j m|
$$

and

$$
\hat{T}(j)_{k Q}=\sum_{m, m^{\prime}=-j}^{j}(-1)^{j-m}\left\langle j m^{\prime} j-m \mid k Q\right\rangle\left|j m^{\prime}\right\rangle_{Z}{ }_{Z}\langle j m|
$$

respectively.

To relate both sets of operators the Eq. (A.5) is substituted in (A.7)

$$
\begin{array}{r}
\hat{T}(j)_{k Q}=\sum_{m, m^{\prime}} \sum_{m_{1}, m_{2}}(-1)^{j-m}\left\langle j m^{\prime} j-m \mid k Q\right\rangle D_{m_{1}, m^{\prime}}^{(j)}(\alpha, \beta, \gamma) \\
D_{m_{2}, m}^{(j) *}(\alpha, \beta, \gamma)\left|j m_{1}\right\rangle_{z}\left\langle j m_{2}\right|
\end{array}
$$

Employing the symmetry of the rotation matrix elements, the Clebsch-Gordan series, the orthogonality of the Clebsch-Gordan coefficients and Eq. (A.6) this cumbersome expression can be transformed into something as simple as

$$
\hat{T}(j)_{k Q}=\sum_{q=-k}^{k} \hat{T}(j)_{k q} D_{q Q}^{(k)}(\alpha, \beta, \gamma)
$$

which sets that the polarisation operators behave as irreducible tensors. The unitarity of the rotation matrix makes possible to invert (A.9) obtaining

$$
\hat{T}(j)_{k q}=\sum_{Q=-k}^{k} \hat{T}(j)_{k Q} D_{q Q}^{(k) *}(\alpha, \beta, \gamma)
$$

It is now quite straightforward to determine the transformation properties of the quantum polarisation moments. Taking the adjoint of (A.10), multiplying both sides by the density operator $\hat{\rho}$, taking the trace and using the Eq. (2.17) we find the 
relation between the statistical tensors in both frames

$$
\left\langle\hat{T}^{\dagger}(j)_{k q}\right\rangle=\sum_{Q=-k}^{k}\left\langle\hat{T}^{\dagger}(j)_{k Q}\right\rangle D_{q Q}^{(k)}(\alpha, \beta, \gamma)
$$

As the statistical tensors are related to the quantum polarisation moments (see Eq. (2.41)), this expression can be rewritten as

$$
a_{q}^{(k)}=\sum_{Q=-k}^{k} A_{Q}^{(k)} D_{q Q}^{(k) *}(\alpha, \beta, \gamma)
$$

providing us with the desired connection between the $x y z$ and the $X Y Z$ moments and showing that they also transform as irreducible tensors.

\section{Classical polarisation moments behaviour under rotations}

The incorporation of any classical moments property in this Appendix, whose title is "Rotations in quantum mechanics", could seem incongruous. The justification for this inclusion stems from the moments definitions as the average of the modified spherical harmonics over the classical PDF

$$
a_{q}^{(k)}=\left\langle C_{k q}\left(\theta_{j}, \phi_{j}\right)\right\rangle
$$

As this equation suggests, to analyse the connection between the classical moments defined in two different frames it will be necessary to know previously what is the relation between the corresponding modified spherical harmonics. These functions are (except a constant factor) the spatial coordinates representation of the $|j m\rangle$ kets $^{2}$ and, in consequence, that relation can be immediately derived from Eq. (A.5)

$$
C_{k q}\left(\theta_{j}, \phi_{j}\right)=\sum_{Q=-k}^{k} C_{k Q}\left(\Theta_{j}, \Phi_{j}\right) D_{q Q}^{(k) *}(\alpha, \beta, \gamma)
$$

where $C_{k q}\left(\theta_{j}, \phi_{j}\right)$ and $C_{k Q}\left(\Theta_{j}, \Phi_{j}\right)$ represent the modified spherical harmonics in the customary $x y z$ and $X Y Z$ reference frames, $\left(\theta_{j}, \phi_{j}\right)$ are the polar angles of an unitary vector $\vec{u}$ in the $x y z$ frame and $\left(\Theta_{j}, \Phi_{j}\right)$ are the polar angles of the same unitary vector in the $X Y Z$ frame.

Multiplying both sides of Eq. (A.14) by the PDF function and by the corresponding

${ }^{2}$ For integer values of $j$ 
volume elements ${ }^{3}\left(\sin \theta_{j} d \theta_{j} d \phi_{j}\right.$ in the $x y z$ frame and $\sin \Theta_{j} d \Theta_{j} d \Phi_{j}$ in the $X Y Z$ frame), integrating over all the spatial directions and using Eq. (2.4) it is obtained the expression

$$
a_{q}^{(k)}=\sum_{Q=-k}^{k} A_{Q}^{(k)} D_{q Q}^{(k) *}(\alpha, \beta, \gamma)
$$

for the connection between the classical polarisation moments in both frames. As in the quantum mechanical case, the classical moments transform under rotation as irreducible tensors.

\footnotetext{
${ }^{3}$ The Jacobian corresponding to the coordinates change caused by a rotation of Euler angles $(\alpha, \beta, \gamma)$ is always one regardless the definite values of the angles. This is so because the coordinates transformation matrix, whose determinant is the Jacobian, is the product of three orthogonal matrices
} 


\section{B. Real polarisation moments}

The mathematical justification for the results included in Sec. 2.4 are now presented. In particular, the discussion will be focused in two points:

- The directional interpretation of the real moments is proved by expressing the classical (quantum mechanical) real moments as the average (expectation value) of a real spherical harmonic (Hermitean operator) directly related to the coordinate system.

- The procedure to calculate the range of accessible values for every real moment is also shown. The limits of these intervals are found to depend on the system population, supporting the necessity for the renormalised moments introduction.

\section{Classical moments}

The explicit expression for the modified spherical harmonics is given by [37]

$$
C_{k q}(\theta, \phi)=e^{i q \phi} \sqrt{\frac{(k-q) !}{(k+q) !}} P_{k q}(\cos \theta)
$$

where $P_{k q}(\cos (\theta))$ represents an associated Legendre polynomial. These polynomials are real functions of the polar angle $\theta$ which solve the associated Legendre differential equation [150].

As was showed in Sec. 2.1, the classical complex polarisation moments can be calculated by averaging the modified spherical harmonics over the polarisation density function (PDF)

$$
a_{q}^{(k)}=\int_{-1}^{1} \int_{0}^{2 \pi} P\left(\theta_{j}, \phi_{j}\right) C_{k q}\left(\theta_{j}, \phi_{j}\right) d \cos \theta_{j} d \phi_{j}=\left\langle C_{k q}\left(\theta_{j}, \phi_{j}\right)\right\rangle
$$

Combining this expression with Eq. (B.1) and (2.55) and taking into account the 
complex exponential definition, the real classical moments can be written as

$$
\begin{aligned}
& a_{q+}^{\{k\}}=\sqrt{2}(-1)^{q} \sqrt{\frac{(k-q) !}{(k+q) !}}\left\langle\cos \left(q \phi_{j}\right) P_{k q}\left(\cos \theta_{j}\right)\right\rangle \quad 1 \leq q \leq k \\
& a_{0}^{\{k\}}=\left\langle P_{k 0}\left(\cos \theta_{j}\right)\right\rangle \\
& a_{q-}^{\{k\}}=\sqrt{2}(-1)^{q} \sqrt{\frac{(k-q) !}{(k+q) !}}\left\langle\sin \left(q \phi_{j}\right) P_{k q}\left(\cos \theta_{j}\right)\right\rangle \quad 1 \leq q \leq k
\end{aligned}
$$

To find the directional meaning corresponding to each moment is now an straightforward task. It is only necessary to remember that the cartesian components $\left(J_{x}, \jmath_{y}\right.$ and $\jmath_{z}$ ) of an unitary vector $(\vec{\jmath})$ pointing along the $\mathbf{j}$ direction are given by

$$
\begin{aligned}
& \jmath_{x}=\sin \theta_{j} \cos \phi_{j} \\
& \jmath_{y}=\sin \theta_{j} \sin \phi_{j} \\
& \jmath_{z}=\cos \theta_{j}
\end{aligned}
$$

and to know the explicit form of the associated Legendre polynomials [150]. For instance, the $k=1$ polynomials are given by

$$
\begin{aligned}
P_{10}\left(\cos \theta_{j}\right) & =\cos \theta_{j} \\
P_{11}\left(\cos \theta_{j}\right) & =-\sin \theta_{j}
\end{aligned}
$$

which, when substituted in Eq. (B.3) provide us with the following expression for the corresponding real moments

$$
\begin{gathered}
a_{1+}^{\{1\}}=\left\langle\cos \phi_{j} \sin \theta_{j}\right\rangle=\left\langle\vec{\jmath}_{x}\right\rangle \\
a_{0}^{\{1\}}=\left\langle\cos \theta_{j}\right\rangle=\left\langle\vec{\jmath}_{z}\right\rangle \\
a_{1-}^{\{1\}}=\left\langle\sin \phi_{j} \sin \theta_{j}\right\rangle=\left\langle\vec{\jmath}_{y}\right\rangle
\end{gathered}
$$

corroborating that the positive (negative) values of the $a_{1+}^{\{1\}}, a_{0}^{\{1\}}$ or $a_{1-}^{\{1\}}$ moments are related to the $\mathbf{j}$ orientation along the positive (negative) $x, z$ or $y$ axis respectively (see Table 2.1). Selecting the corresponding Legendre polynomial it is possible to extend this procedure to higher values of $k$.

The range of accessible values for every real moment can be simply determined from Eq. (B.3) by substituting the Legendre polynomial and the sine and cosine functions by their maximum or minimum values. As before, the $k=1$ moments are used to 
illustrate the process. In this case

$$
-1 \leq P_{1 q}\left(\cos \theta_{j}\right) \leq 1
$$

and

$$
-\langle 1\rangle \leq a_{1 \pm, 0}^{\{1\}} \leq\langle 1\rangle
$$

where $\langle 1\rangle$ represents the $a_{0}^{\{0\}}$ moment $\left(P_{00}\left(\cos \theta_{j}\right)=1\right)$

$$
\langle 1\rangle=\int_{-1}^{1} \int_{0}^{2 \pi} P\left(\theta_{j}, \phi_{j}\right) d \cos \theta_{j} d \phi_{j}
$$

that is, the system population. Its value uses to be chosen as one (the PDF is normalised to unity). In such a case the $k=1$ moments values will be contained in the $[-1,1]$ interval. Otherwise the longitude of this interval will depend on the population according to (B.6). This procedure for setting the interval limits (and the limits dependence on the population) holds for all the moments.

\section{Quantum mechanical moments}

The starting point for the quantum mechanical analysis of the real moments are the $\hat{J}_{q}^{(k)}$ spherical operators 33,38 . For $k=0$ and $k=1$ rank they are defined as 38

$$
\hat{J}_{0}^{(0)}=\hat{1}
$$

and

$$
\begin{gathered}
\hat{J}_{1}^{(1)}=-\frac{1}{\sqrt{2}}\left(\hat{J}_{x}+i \hat{J}_{y}\right)=-\frac{1}{\sqrt{2}} \hat{J}_{+} \\
\hat{J}_{0}^{(1)}=\hat{J}_{z} \\
\hat{J}_{-1}^{(1)}=\frac{1}{\sqrt{2}}\left(\hat{J}_{x}-i \hat{J}_{y}\right)=\frac{1}{\sqrt{2}} \hat{J}_{-}
\end{gathered}
$$

while those corresponding to $k \geq 2$ can be obtained by contracting two operators of lower range

$$
\hat{J}_{q}^{(k)}=\left[\hat{J}^{(k-1)} \otimes \hat{J}^{(1)}\right]_{q}^{(k)}=\sum_{q_{1}=-(k-1)}^{k-1}\left\langle(k-1) q_{1} 1 q_{2} \mid k q\right\rangle \hat{J}_{q_{1}}^{(k-1)} \hat{J}_{q_{2}}^{(1)}
$$

The following relation for the adjoint of the $\hat{J}_{q}^{(k)}$ operators

$$
\hat{J}_{q}^{(k) \dagger}=(-1)^{q} \hat{J}_{-q}^{(k)}
$$


can be demonstrated employing mathematical induction (the equality holds for the $k=1$ operators (see Eq. (B.9) ) and, supposing that it is valid when the rank is $k-1$, the Eq. (B.10) can be used to prove that it also holds for rank $k$ ). This expression implies that, unless $q=0, \hat{J}_{q}^{(k)}$ is not Hermitean.

The importance of these operators lies in their relation to the quantum mechanical polarisation moments. Applying Eq. (2.17) and the completeness of the $|j m\rangle$ basis it is possible to express their expectation values as

$$
\left\langle\hat{J}_{q}^{(k)}\right\rangle=\operatorname{tr}\left(\rho J_{q}^{(k)}\right)=\sum_{m, m^{\prime}=-j}^{j}\left\langle j m^{\prime}|\rho| j m\right\rangle\left\langle j m\left|\hat{J}_{q}^{(k)}\right| j m^{\prime}\right\rangle
$$

As the $\hat{J}_{q}^{(k)}$ operators behave as irreducible tensors, the second term in the sum can be evaluated by means of the Wigner-Eckart theorem 37, 38

$$
\left\langle j m\left|\hat{J}_{q}^{(k)}\right| j m^{\prime}\right\rangle=(-1)^{2 k}\left\langle j m^{\prime} k q \mid j m\right\rangle \frac{\left\langle j|| \hat{J}^{k}|| j\right\rangle}{\sqrt{2 j+1}}
$$

where the fraction numerator represents the reduced matrix element which depends exclusively on $k$ and on the quantum number $j$ (explicit expressions for different $\mathrm{k}$ values can be found in [38). Substituting this expression in Eq. (B.12) and comparing with Eq. (2.43) it is obtained the following expression

$$
\begin{aligned}
\left\langle\hat{J}_{q}^{(k)}\right\rangle & =\frac{\left\langle j\left\|\hat{J}^{k}\right\| j\right\rangle}{\sqrt{2 j+1}} \sum_{m, m^{\prime}=-j}^{j}\left\langle j m^{\prime}|\rho| j m\right\rangle\left\langle j m^{\prime} k q \mid j m\right\rangle \\
& =\underbrace{\frac{\left\langle j\left\|\hat{J}^{k}\right\| j\right\rangle}{\sqrt{2 j+1}} a_{q}^{(k)}}_{\text {depends on } k \text { and } j}
\end{aligned}
$$

that relates the complex quantum mechanical moments with the expectation values of the $\hat{J}_{q}^{(k)}$ operators.

Combining Eq. (B.14) with Eq. (2.54) it is possible to express the real moments in terms of the spherical $\hat{J}_{q}^{(k)}$ operators

$$
\begin{aligned}
a_{q+}^{\{k\}} & =c_{k, j}\left\langle\hat{J}_{q+}^{\{k\}}\right\rangle \quad 1 \leq q \leq k \\
a_{0}^{\{k\}} & =c_{k, j}\left\langle\hat{J}_{0}^{\{k\}}\right\rangle \\
a_{q-}^{\{k\}} & =c_{k, j}\left\langle\hat{J}_{q-}^{\{k\}}\right\rangle \quad 1 \leq q \leq k
\end{aligned}
$$


where $c_{k, j}$ represents the factor

$$
\frac{\sqrt{2 j+1}}{\left\langle j\left\|\hat{J}^{k}\right\| j\right\rangle}
$$

and a new set of operators have been defined

$$
\begin{aligned}
\hat{J}_{q+}^{\{k\}} & =\frac{1}{\sqrt{2}}\left[(-1)^{q} \hat{J}_{q}^{(k)}+\hat{J}_{-q}^{(k)}\right] \quad 1 \leq q \leq k \\
\hat{J}_{0}^{\{k\}} & =\hat{J}_{0}^{(k)} \\
\hat{J}_{q-}^{\{k\}} & =\frac{1}{i \sqrt{2}}\left[(-1)^{q} \hat{J}_{q}^{(k)}-\hat{J}_{-q}^{(k)}\right] \quad 1 \leq q \leq k
\end{aligned}
$$

which are all of them Hermitean.

The directional meaning of the real moments can now be determined. As in the classical case, we will use the $k=1$ rank moments to illustrate the procedure (it can be immediately extended to higher values of the rank). Eqs. (B.9) and (B.17) show that, in this case

$$
\hat{J}_{1+}^{\{1\}}=\hat{J}_{x} \quad \hat{J}_{0}^{\{1\}}=\hat{J}_{z} \quad \hat{J}_{1-}^{\{1\}}=\hat{J}_{y}
$$

and from 38

$$
c_{1, j}=\frac{1}{\sqrt{j(j+1)}}
$$

In consequence,

$$
\begin{aligned}
& a_{1+}^{\{1\}}=\frac{\left\langle\hat{J}_{x}\right\rangle}{\sqrt{j(j+1)}} \\
& a_{0}^{\{1\}}=\frac{\left\langle\hat{J}_{z}\right\rangle}{\sqrt{j(j+1)}} \\
& a_{1-}^{\{1\}}=\frac{\left\langle\hat{J}_{y}\right\rangle}{\sqrt{j(j+1)}}
\end{aligned}
$$

As expected, the relation between directions and moments that stems from this equations coincides with that obtained in the classical case: positive (negative) values of the $a_{1+}^{\{1\}}, a_{0}^{\{1\}}$ or $a_{1-}^{\{1\}}$ moments represent a $\mathbf{j}$ orientation along the positive (negative) $x, z$ or $y$ axis respectively.

One strategy to evaluate the upper and lower limits of the accessible range of values for each real moment is based on (i) determining the extreme values for $\left\langle\hat{J}_{q \pm, 0}^{\{k\}}\right\rangle$ and (ii) substituting them into Eq. (B.15). For instance, the expectation value of the $\hat{J}_{1 \pm, 0}^{\{1\}}$ operators fulfill

$$
\left|\left\langle\hat{J}_{1 \pm, 0}^{\{1\}}\right\rangle\right| \leq j\langle\hat{1}\rangle
$$


where $\langle\hat{1}\rangle=\operatorname{tr}(\rho)$ is the system population. Therefore,

$$
-\frac{j}{\sqrt{j(j+1)}}\langle\hat{1}\rangle=-\left(j c_{1, j}\right)\langle\hat{1}\rangle \leq a_{1 \pm, 0}^{\{1\}} \leq j c_{1, j}\langle\hat{1}\rangle=\frac{j}{\sqrt{j(j+1)}}\langle\hat{1}\rangle
$$

As expected, depending on wether the population is normalised to unity or not, the length of the interval will change.

This procedure for determining the limits of the real moments becomes cumbersome and difficult to apply as $j$ increases. A more practical approach to the problem consists of employing the fact that the real moments are defined as the expectation values of Hermitean operators whose explicit expression can be obtained through combination of Eqs. (2.41) and (2.54):

$$
\begin{array}{rr}
(-1)^{q}\left(\frac{2 j+1}{2(2 k+1)}\right)^{1 / 2}\left((-1)^{q} \hat{T}^{\dagger}(j)_{k-q}+\hat{T}^{\dagger}(j)_{k q}\right) & \text { for } a_{q+}^{\{k\}} \text { moments } \\
\left(\frac{2 j+1}{2 k+1}\right)^{1 / 2} \hat{T}^{\dagger}(j)_{k 0} & \text { for } a_{0}^{\{k\}} \text { moments } \\
\frac{(-1)^{q}}{i}\left(\frac{2 j+1}{2(2 k+1)}\right)^{1 / 2}\left((-1)^{q} \hat{T}^{\dagger}(j)_{k-q}-\hat{T}^{\dagger}(j)_{k q}\right) & \text { for } a_{q-}^{\{k\}} \text { moments }
\end{array}
$$

The maximum and minimum eigenvalues of each of these operators will coincide with the limit values of the corresponding real moment (assuming that the system population is one). Such eigenvalues can be evaluated by diagonalising the representation of the operators in the $|j m\rangle$ basis:

$$
\begin{gathered}
(-1)^{q} \frac{2 j+1}{2(2 k+1)} \underbrace{1 / 2}_{A_{m_{1} m_{2}}} \underbrace{(-1)^{j-m_{1}}\left((-1)^{q}\left\langle j m_{2} j-m_{1} \mid k-q\right\rangle+\left\langle j m_{2} j-m_{1} \mid k q\right\rangle\right)}_{A_{m_{1} m_{2}}} \quad\left(a_{q+}^{\{k\}}\right) \\
\frac{2 j+1}{2 k+1} \underbrace{(-1)^{q}}_{A^{\prime \prime}{ }_{m_{1} m_{2}} \underbrace{(-1)^{j-m_{1}}\left\langle j m_{2} j-m_{1} \mid k 0\right\rangle}} \\
\frac{2 j+1}{2(2 k+1)} \underbrace{1 / 2}_{\left.a_{0}^{\{k\}}\right)} \underbrace{(-1)^{j-m_{1}}\left((-1)^{q}\left\langle j m_{2} j-m_{1} \mid k-q\right\rangle-\left\langle j m_{2} j-m_{1} \mid k q\right\rangle\right)}\left(a_{q-}^{\{k\}}\right)
\end{gathered}
$$

where Eq. (2.24) (the definition of the $\hat{T}(j)_{k q}$ operators) has been used. The elements $A_{m 1, m 2}$ correspond to an hermitic matrix, $A_{m 1, m 2}^{\prime}$ to a diagonal matrix and $A_{m 1, m 2}^{\prime \prime}$ to an anti-hermitic matrix. Diagonalisation of these three matrices ${ }^{1}$, selection of the maximum/minimum eigenvalue and consideration of the additional phases and

\footnotetext{
${ }^{1} \mathrm{~A}$ discussion about how to perform such diagonalisation, together with suitable fortran77 subroutines, can be found in reference 69
} 


\begin{tabular}{c|ccccccccc}
\hline \hline$j$ & $a_{0}^{1}$ & $a_{1 \pm}^{1}$ & $a_{0}^{2}$ & $a_{1 \pm}^{2}$ & $a_{2 \pm}^{2}$ & $a_{0}^{3}$ & $a_{1 \pm}^{3}$ & $a_{2 \pm}^{3}$ & $a_{3 \pm}^{3}$ \\
\hline $1 / 2$ & 0.577 & 0.577 & - & - & - & - & - & - & - \\
1 & 0.707 & 0.707 & 0.316 & 0.548 & 0.548 & - & - & - & - \\
$3 / 2$ & 0.775 & 0.775 & 0.447 & 0.447 & 0.447 & 0.507 & 0.523 & 0.378 & 0.535 \\
2 & 0.816 & 0.816 & 0.535 & 0.535 & 0.535 & 0.535 & 0.500 & 0.598 & 0.423 \\
$5 / 2$ & 0.845 & 0.845 & 0.598 & 0.548 & 0.548 & 0.483 & 0.448 & 0.463 & 0.436 \\
3 & 0.866 & 0.866 & 0.645 & 0.589 & 0.589 & 0.408 & 0.408 & 0.408 & 0.408 \\
$7 / 2$ & 0.882 & 0.882 & 0.683 & 0.612 & 0.612 & 0.461 & 0.458 & 0.441 & 0.446 \\
4 & 0.894 & 0.894 & 0.714 & 0.637 & 0.637 & 0.505 & 0.479 & 0.483 & 0.477 \\
$9 / 2$ & 0.905 & 0.905 & 0.739 & 0.655 & 0.655 & 0.542 & 0.496 & 0.464 & 0.489 \\
5 & 0.913 & 0.913 & 0.760 & 0.672 & 0.672 & 0.574 & 0.518 & 0.461 & 0.506 \\
\hline \hline
\end{tabular}

Table B.1.: Upper limit of the $k=1-3$ real polarisation moments for $j$ values comprised in the interval going from $\frac{1}{2}$ to 5 .

\begin{tabular}{c|ccccccccc}
\hline \hline$j$ & $a_{0}^{1}$ & $a_{1 \pm}^{1}$ & $a_{0}^{2}$ & $a_{1 \pm}^{2}$ & $a_{2 \pm}^{2}$ & $a_{0}^{3}$ & $a_{1 \pm}^{3}$ & $a_{2 \pm}^{3}$ & $a_{3 \pm}^{3}$ \\
\hline $1 / 2$ & -0.577 & -0.577 & - & - & - & - & - & - & - \\
1 & -0.707 & -0.707 & -0.632 & -0.548 & -0.548 & - & - & - & - \\
$3 / 2$ & -0.775 & -0.775 & -0.447 & -0.447 & -0.447 & -0.507 & -0.523 & -0.378 & -0.535 \\
2 & -0.816 & -0.816 & -0.535 & -0.535 & -0.535 & -0.535 & -0.500 & -0.598 & -0.423 \\
$5 / 2$ & -0.845 & -0.845 & -0.478 & -0.548 & -0.548 & -0.483 & -0.448 & -0.463 & -0.436 \\
3 & -0.866 & -0.866 & -0.516 & -0.589 & -0.589 & -0.408 & -0.408 & -0.408 & -0.408 \\
$7 / 2$ & -0.882 & -0.882 & -0.488 & -0.612 & -0.612 & -0.461 & -0.458 & -0.441 & -0.446 \\
4 & -0.894 & -0.894 & -0.510 & -0.637 & -0.637 & -0.505 & -0.479 & -0.483 & -0.477 \\
$9 / 2$ & -0.905 & -0.905 & -0.492 & -0.655 & -0.655 & -0.542 & -0.496 & -0.464 & -0.489 \\
5 & -0.913 & -0.913 & -0.506 & -0.672 & -0.672 & -0.574 & -0.518 & -0.461 & -0.506 \\
\hline \hline
\end{tabular}

Table B.2.: Lower limit of the $k=1-3$ real polarisation moments for $j$ values comprised in the interval going from $\frac{1}{2}$ to 5 .

constant factors included in the former Equations lead us to Tables B.1 and B.2, which contain the limits for the $k=1-3$ moments when the value of $j$ goes from one half to five. It is necessary to point out that these Tables hold for systems with population one.

The quantum mechanical introduction of the real moments could not finish without pointing out a subtle characteristic which does not appear in the classical case. The complex quantum mechanical $a_{q}^{(k)}$ moments are defined as the expectation value of a non Hermitean operator and, for that reason, they are not observable quantities (except when $q=0$ ). On the contrary, their real counterparts correspond to the expectation value of an Hermitean operator and, therefore, they are experimentally measurable. 


\section{Observable DCS}

Eq. (4.20) was the basis for the analysis of reaction control through the reactants polarisation. This Appendix provides with its derivation, both in the classical and in the quantum mechanical frame.

\section{Classical mechanics}

The classical proof of Eq. (4.20) stars with the classical probability density function $P_{r}\left(\theta, \theta_{j}, \phi_{j}\right)$ introduced in Sec. 4.3. This PDF (an intrinsic reaction property) gives the probability of observing reactive scattering from a given reactants rovibrational state into a given products rovibrational state and at scattering angle $\theta$ when the initial direction of the $\mathrm{BC}$ reactant rotational angular momentum is the one associated with the spherical angles $\theta_{j}$ and $\phi_{j}$. The classical reaction PDF can be expanded as a series of modified spherical harmonics [37] in the form

$$
P_{r}\left(\theta, \theta_{j}, \phi_{j}\right)=\sum_{k=0}^{\infty} \sum_{q=-k}^{k} \frac{2 k+1}{4 \pi} S_{q}^{(k)}(\theta) C_{k q}^{*}\left(\theta_{j}, \phi_{j}\right)
$$

where the $S_{q}^{(k)}(\theta)$ 's $\theta$-dependent expansion coefficients are the intrinsic reactants PDDCSs (Sec. 4.3.1). This PDF will be assumed to be normalized to unity

$$
\int_{-1}^{1} \int_{0}^{2 \pi} \int_{-1}^{1} P_{r}\left(\theta, \theta_{j}, \phi_{j}\right) d \cos \theta_{j} d \phi_{j} d \cos \theta=\int_{-1}^{1} S_{0}^{(0)}(\theta) d \cos \theta=1
$$

(the orthogonality of the spherical harmonics (Eq. (2.3)) has been used to perform the integration over $\theta_{j}$ and $\phi_{j}$ ).

The classical PDF (C.1) represents the reactants polarisation when the reaction takes place, irrespective of the initial preparation of the directions of $\mathbf{j}$. However, when the directions of the angular momentum are prepared prior to the collision, one must consider the actual polarisation of reactants. This is an extrinsic property: instead of depending on the reaction dynamics it depends on external intervention. The extrinsic polarisation of the BC molecule can be described by a PDF (the extrinsic 
reactants $\mathrm{PDF}$ ) that can be expanded in a multipolar series entirely similar to C.1

$$
\rho\left(\theta_{j}, \phi_{j}\right)=\sum_{k=0}^{\infty} \sum_{q=-k}^{k} \frac{2 k+1}{4 \pi} a_{q}^{(k)} C_{k q}^{*}\left(\theta_{j}, \phi_{j}\right)
$$

where $a_{q}^{(k)}$ are the extrinsic polarisation moments. As its intrinsic counterpart, the extrinsic PDF will also be normalised to unity

$$
\int_{-1}^{1} \int_{0}^{2 \pi} \rho\left(\theta_{j}, \phi_{j}\right) d \cos \theta_{j} d \phi_{j}=a_{0}^{(0)}=1
$$

The reaction differential cross section must take into account the actual, extrinsic reactant preparation scheme as well as the intrinsic properties of the reaction. The suitable way of performing this combination of extrinsic and intrinsic information is based on the joint PDF obtained by multiplying Eq. ((区.1) and Eq. ((C.3)

$$
P_{r}\left(\theta, \theta_{j}, \phi_{j}, \alpha, \beta, \gamma\right)=\sum_{k, q, k^{\prime}, q^{\prime}} \frac{(2 k+1)\left(2 k^{\prime}+1\right)}{(4 \pi)^{2}} S_{q}^{(k) *}(\theta) a_{q^{\prime}}^{\left(k^{\prime}\right)} C_{k q}\left(\theta_{j}, \phi_{j}\right) C_{k^{\prime} q^{\prime}}^{*}\left(\theta_{j}, \phi_{j}\right)
$$

which gives the probability that the reaction products appear at an scattering angle $\theta$, when $\mathbf{j}$ points in the direction given by $\theta_{j}$ and $\phi_{j}$ and the extrinsic polarisation of the $\mathrm{BC}$ molecule is that corresponding to the $a_{q}^{(k)}$ moments (as it was discussed in Sec. 4.4, their concrete values depend on the $\alpha, \beta$ and $\gamma$ Euler angles which determine the LAB frame position with respect to the center-of-mass frame). By integrating the reaction probability for all the possible directions of $\mathbf{j}$ (and employing again the modified spherical harmonics orthogonality) it is obtained a second joint PDF

$$
P_{r}(\theta, \alpha, \beta, \gamma)=\sum_{k=0}^{\infty} \sum_{q=-k}^{k} \frac{(2 k+1)}{(4 \pi)} S_{q}^{(k) *}(\theta) a_{q}^{(k)}
$$

representing the probability of forming the products with scattering angle $\theta$ when the extrinsic polarisation of the reactants is given by the $a_{q}^{(k)}$ moments.

The differential cross section can be expressed in terms of $P_{r}(\theta, \alpha, \beta, \gamma)$ as

$$
\frac{d \sigma}{d \omega}=\frac{\sigma_{\text {iso }}}{2 \pi} \frac{P_{r}(\theta, \alpha, \beta, \gamma)}{1 / 4 \pi}=\frac{\sigma_{\text {iso }}}{2 \pi} \sum_{k=0}^{\infty} \sum_{q=-k}^{k}(2 k+1) S_{q}^{(k) *}(\theta) a_{q}^{(k)}
$$

where $\sigma_{\text {iso }}$ is the integral cross section of the reaction involving unpolarized reactants (which must be divided by $2 \pi$ in order to take into account the fact that the isotropic 
differential cross section is independent of the azimuthal angle) and the $4 \pi$ factor in the denominator ensures its correct normalisation (its origin is the following: if the reactants are unpolarised prior to reaction the differential cross section can be written as 1 ]

$$
\frac{d \sigma}{d \omega}=\frac{d \sigma_{\text {iso }}}{d \omega}=\frac{\sigma_{\text {iso }}}{2 \pi} S_{0}^{(0)}(\theta)
$$

(remember that $S_{0}^{(0)}(\theta)$ coincides with the angular distribution for unpolarised reactants). On the other side, the polarisation moments fulfill $a_{q}^{(k)}=\delta_{k 0} \delta_{q 0}$ (see Sec. 2.3.3) and, from this fact, it ensues that: (i) $P_{r}(\theta, \alpha, \beta, \gamma)$ will only depend on $\theta$, (ii) it can be written as

$$
\frac{S_{0}^{(0)}(\theta)}{4 \pi}
$$

and (iii) its integral over the scattering angle is $1 /(4 \pi)$ (see Eq. (C.2)). So, dividing $P_{r}(\theta, \alpha, \beta, \gamma)$ by $1 /(4 \pi)$ ensures that it is normalised to unity when the reactants are unpolarised and that Eq. (C.7) provides with the right value of the DCS).

\section{Quantum mechanics}

The starting point for the quantum mechanical prove of Eq. (4.20) is the expression [24]

$$
\frac{d \sigma}{d \omega}=\sum_{\Omega^{\prime} \Omega_{1} \Omega_{2}} f_{\Omega^{\prime} \Omega_{1}}(\theta) f_{\Omega^{\prime} \Omega_{2}}^{*}(\theta)\left\langle j \Omega_{1}|\hat{\rho}| j \Omega_{2}\right\rangle
$$

which represents the state-to-state differential cross section for an arbitrary polarization of the reactants (and therefore arbitrary coherence between the possible reactant helicities $(\Omega)$ ). The density operator $\hat{\rho}$ describes the extrinsic preparation of the reactants and $\left\langle j \Omega_{1}|\hat{\rho}| j \Omega_{2}\right\rangle$ is an element of its matrix representation in the $|j \Omega\rangle$ basis. The $f_{\Omega^{\prime} \Omega}(\theta)$ function represents the scattering amplitude. It can be written in terms of the scattering matrix in the helicity representation as 74

$$
f_{\Omega^{\prime} \Omega}(\theta)=\frac{1}{2 i k_{\mathrm{in}}} \sum_{J}(2 J+1) d_{\Omega^{\prime} \Omega}^{J}(\theta) S_{j^{\prime} \Omega^{\prime}, j \Omega}^{J}
$$

where $d_{\Omega^{\prime} \Omega}^{J}(\theta)$ is a reduced rotation matrix element [37, $k_{\text {in }}$ the wavenumber associated with the incoming (reactants) plane wave and $S_{j^{\prime} \Omega^{\prime}, j \Omega}^{J}$ is an element of the $\mathrm{S}$ matrix in the helicity representation (for a discussion about the meaning of the different labels see Sec. 3.4 or Sec. 4.3.5).

It is necessary to point out three characteristics of this formula, namely (i) that the product polarization is being disregarded (the DCS is summed over product helicities), (ii) that the reactants are being assumed to be in a well-defined rotational energy 
level, so that the only coherences playing any role are those among different helicity substates, and (iii) that the DCS formula mixes intrinsic (scattering amplitudes) and extrinsic (reactants density matrix) properties.

The matrix elements of the extrinsic $\hat{\rho}$ operator can be written as (Eq. (2.42))

$$
\left\langle j \Omega_{1}|\rho| j \Omega_{2}\right\rangle=\sum_{k=0}^{2 j} \sum_{q=-k}^{q} \frac{2 k+1}{2 j+1} a_{q}^{(k)}\left\langle j \Omega_{1}, k q \mid j \Omega_{2}\right\rangle
$$

where $a_{q}^{(k)}$ are the quantum mechanical moments describing the extrinsic polarisation of the reactants.

Inserting $\overline{\text { C.12 }}$ into Eq. (C.10), multiplying the right hand side of the resulting expression by

$$
\frac{\sigma_{i s o}}{2 p i} \frac{2 p i}{\sigma_{i s o}}=1
$$

( $\sigma_{i s o}$ is again the state-to-state integral cross section when the reactants are initially unpolarised) and taking into account that $\sigma_{i s o}$ is given by 74

$$
\sigma_{\text {iso }}=\frac{\pi}{(2 j+1) k_{\text {in }}^{2}} \sum_{J \Omega^{\prime} \Omega}(2 J+1)\left|S_{j^{\prime} \Omega^{\prime}, j \Omega}^{J}\right|^{2},
$$

allows for obtaining the desired expression for the differential cross section

$$
\frac{d \sigma}{d \omega}=\frac{\sigma_{\text {iso }}}{2 \pi} \sum_{k=0}^{2 j} \sum_{q=-k}^{k}(2 k+1) S_{q}^{(k) *}(\theta) a_{q}^{(k)}
$$

with the quantum mechanical PDDCSs given by Eq. (4.17). 


\section{Molecular axis portraits}

Let us suppose that the state of a diatomic molecule is represented by the density operator $\hat{\rho}$. All the information regarding the molecule is contained in this operator. In particular: (i) Eq. 2.43 can be used to determine the moments which describe the polarisation of the molecule rotational angular momentum (j) and (ii) the $\mathbf{j}$ spatial distribution corresponding to these moments can be determined by means of the quantum PDF introduced in Sec. 2.5.

The position of the molecule internuclear axis $(\mathbf{r})$ is not independent on $\mathbf{j}$ position (thinking in classical terms, $\mathbf{r}$ is rotating perpendicularly to $\mathbf{j}$ ) and, therefore, any nonisotropic $\mathbf{j}$ distribution will give rise to a non-isotropic distribution of the internuclear axis. The goal of this Appendix is to obtain an explicit expression for the internuclear axis distribution when the diatomic molecule is in a defined rotational state. In this case, $\hat{\rho}$ can be written as

$$
\hat{\rho}=\sum_{\Omega_{1}, \Omega_{2}=-j}^{j}\left\langle j \Omega_{1}|\hat{\rho}| j \Omega_{2}\right\rangle\left|j \Omega_{1}\right\rangle\left\langle j \Omega_{2}\right|
$$

where $\Omega$ labels the reactants helicity and the completeness of the $|j \Omega\rangle$ basis has been used.

Assuming that the trace of the density matrix is equal to one

$$
\operatorname{tr}(\rho)=1
$$

and representing by $\left|\theta_{r} \phi_{r}\right\rangle$ an state with fixed internuclear axis pointing along the direction given by the polar angle $\theta_{r}$ and the azimuthal angle $\phi_{r}^{1}$, the diagonal element

$$
\left\langle\theta_{r} \phi_{r}|\hat{\rho}| \theta_{r} \phi_{r}\right\rangle
$$

is the probability of finding $\mathbf{r}$ pointing along the direction given by $\theta_{r}$ and $\phi_{r}$ when

\footnotetext{
${ }^{1}$ As usual, both angles will be considered as referred to the centre-of-mass frame. However, this restriction is not esential for the reasoning and the only condition that they must necessary fulfill is that they are defined in the same frame that the polarisation moments employed to generate the portrait
} 
the state of the molecule is given by $\hat{\rho}$.

By combining Eqs. (D.1) and (D.3) with the definition of the modified spherical harmonics and with Eq. (2.42) it is found the following expression for the portrait

$$
\begin{aligned}
P_{r}\left(\theta_{r}, \phi_{r}\right) & =\left\langle\theta_{r} \phi_{r}|\hat{\rho}| \theta_{r} \phi_{r}\right\rangle \\
& =\sum_{\Omega_{1}, \Omega_{2}}\left\langle j \Omega_{1}|\hat{\rho}| j \Omega_{2}\right\rangle\left\langle\theta_{r} \phi_{r} \mid j \Omega_{1}\right\rangle\left\langle j \Omega_{2} \mid \theta_{r} \phi_{r}\right\rangle \\
& =\sum_{\Omega_{1}, \Omega_{2}}\left\langle j \Omega_{1}|\hat{\rho}| j \Omega_{2}\right\rangle Y_{j \Omega_{1}}\left(\theta_{r} \phi_{r}\right) Y_{j \Omega_{2}}^{*}\left(\theta_{r} \phi_{r}\right) \\
& =4 \pi \sum_{k q} \sum_{\Omega_{1}, \Omega_{2}}(2 k+1) b_{q}^{(k)}\left\langle j \Omega_{1} k q \mid j \Omega_{2}\right\rangle C_{j \Omega_{1}}\left(\theta_{r} \phi_{r}\right) C_{j \Omega_{2}}^{*}\left(\theta_{r} \phi_{r}\right) \text { (D.4) }
\end{aligned}
$$

where $b_{q}^{(k)}$ label the polarisation moments. This equation can be further simplified by means of the Clebsch-Gordan series and the Clebsch-Gordan properties (symmetry and orthogonality) [37, obtaining

$$
P_{r}\left(\theta_{r}, \phi_{r}\right)=\sum_{k=0}^{2 j} \sum_{q=-k}^{k} \frac{2 k+1}{4 \pi} b_{q}^{(k)}\langle j 0, k 0 \mid j 0\rangle C_{k q}^{*}\left(\theta_{r}, \phi_{r}\right)
$$

which is the desired expression.

The molecular axis portraits can be used to visualise the $\mathbf{r}$ distributions corresponding to any set of polarisation moments. When employed in combination with the intrinsic moments the portraits will represent the axis distributions when the reaction takes place and, when generated from extrinsic moments, they will describe the experimental preparation of the reactants. 


\section{E. Derivation of Eq.(7.18)}

The present Appendix provides with a derivation of Eq. (7.18). For the sake of simplicity, we will assume that all the angular momenta quantum numbers are integer. Our starting point will be the Clebsch-Gordan series [37]:

$$
D_{M_{1} N_{1}}^{J_{1}}(\alpha \beta \gamma) D_{M_{2} N_{2}}^{J_{2}}(\alpha \beta \gamma)=\sum_{J=\left|J_{1}-J_{2}\right|}^{J_{1}+J_{2}} \sum_{M N} C_{J_{1} M_{1} J_{2} M_{2}}^{J M} D_{M N}^{J}(\alpha \beta \gamma) C_{J_{1} N_{1} J_{2} N_{2}}^{J N}
$$

where the following notation for the Clebsch-Gordan coefficients has been used

$$
C_{J_{1} M_{1} J_{2} M_{2}}^{J M}=\left\langle J_{1} M_{1} J_{2} M_{2} \mid J M\right\rangle
$$

in order to reduce the room occupied by the different Equations.

Multiplying both sides of Eq. (E.1) by $C_{J_{1} N_{1} J_{2} N_{2}}^{J^{\prime} N^{\prime}}$, summing over $N_{1}$ and $N_{2}$ and employing the orthogonality of the Clebsch-Gordan coefficients [37] we obtain

$$
\sum_{N_{1} N_{2}} D_{M_{1} N_{1}}^{J_{1}}(\alpha \beta \gamma) D_{M_{2} N_{2}}^{J_{2}}(\alpha \beta \gamma) C_{J_{1} N_{1} J_{2} N_{2}}^{J N}=\sum_{M} C_{J_{1} M_{1} J_{2} M_{2}}^{J M} D_{M N}^{J}(\alpha \beta \gamma)
$$

If the Euler angles $\alpha$ and $\gamma$ are fixed as 0, Eq. (E.3) reduces to

$$
\sum_{N_{1} N_{2}} d_{M_{1} N_{1}}^{J_{1}}(\beta) d_{M_{2} N_{2}}^{J_{2}}(\beta) C_{J_{1} N_{1} J_{2} N_{2}}^{J N}=\sum_{M} C_{J_{1} M_{1} J_{2} M_{2}}^{J M} d_{M N}^{J}(\beta)
$$

The symmetry of the Clebsch-Gordan coefficients [37]

$$
\begin{aligned}
C_{J_{1} N_{1} J_{2} N_{2}}^{J N} & =(-1)^{J_{1}-N_{1}} \sqrt{\frac{2 J+1}{2 J_{2}+1}} C_{J_{1} N_{1} J-N}^{J_{2}-N_{2}} \\
& =(-1)^{J-N_{1}-J_{2}} \sqrt{\frac{2 J+1}{2 J_{2}+1}} C_{J_{1}-N_{1} J N}^{J_{2} N_{2}} \\
C_{J_{1} M_{1} J_{2} M_{2}}^{J M} & =(-1)^{J_{1}-M_{1}} \sqrt{\frac{2 J+1}{2 J_{2}+1}} C_{J_{1} M_{1} J-M}^{J_{2}-M_{2}} \\
& =(-1)^{J-M_{1}-J_{2}} \sqrt{\frac{2 J+1}{2 J_{2}+1}} C_{J_{1}-M_{1} J M}^{J_{2} M_{2}}
\end{aligned}
$$


can be used to rewrite Eq. (E.4) as

$$
\sum_{N_{1} N_{2}} d_{M_{1} N_{1}}^{J_{1}}(\beta) d_{M_{2} N_{2}}^{J_{2}}(\beta)(-1)^{N_{1}} C_{J_{1}-N_{1} J N}^{J_{2} N_{2}}=(-1)^{M_{1}} \sum_{M} C_{J_{1}-M_{1} J M}^{J_{2} M_{2}} d_{M N}^{J}(\beta)
$$

As $N_{1}$ is a dump index, it can be substituted by $-N_{1}$ in the first term of the previous equation. This change leads us to

$$
\sum_{N_{1} N_{2}} d_{M_{1}-N_{1}}^{J_{1}}(\beta) d_{M_{2} N_{2}}^{J_{2}}(\beta)(-1)^{N_{1}} C_{J_{1} N_{1} J N}^{J_{2} N_{2}}=(-1)^{M_{1}} \sum_{M} C_{J_{1}-M_{1} J M}^{J_{2} M_{2}} d_{M N}^{J}(\beta)
$$

that, after using the reduced rotation matrices symmetry [37]

$$
d_{M_{1}-N_{1}}^{J_{1}}(\beta)=(-1)^{M_{1}-N_{1}} d_{-M_{1} N_{1}}^{J_{1}}(\beta)
$$

transforms into

$$
\sum_{N_{1} N_{2}} d_{-M_{1} N_{1}}^{J_{1}}(\beta) d_{M_{2} N_{2}}^{J_{2}}(\beta) C_{J_{1} N_{1} J N}^{J_{2} N_{2}}=\sum_{M} C_{J_{1}-M_{1} J M}^{J_{2} M_{2}} d_{M N}^{J}(\beta)
$$

By writing $-M_{1}$ instead of $M_{1}$ in this last expression we recover Eq. (7.18)

$$
\sum_{N_{1} N_{2}} d_{M_{1} N_{1}}^{J_{1}}(\beta) d_{M_{2} N_{2}}^{J_{2}}(\beta) C_{J_{1} N_{1} J N}^{J_{2} N_{2}}=\sum_{M} C_{J_{1} M_{1} J M}^{J_{2} M_{2}} d_{M N}^{J}(\beta),
$$

completing the derivation. 


\section{F. Estereodinámica de reacciones elementales: efecto de la polarización del momento angular rotacional de los reactivos}

\section{F.1. Introducción}

El conocimiento detallado de la influencia de los efectos direccionales sobre las magnitudes observables de una reacción química tiene una importancia fundamental a la hora de comprender el mecanismo del proceso y las fuerzas que dan lugar al mismo. Además, la consideración explícita de tales efectos permite desarrollar técnicas para el control de las colisiones moleculares.

Existen, sin embargo, pocos estudios que proporcionen un marco teórico general para el estudio de la dependencia direccional de las reacciones químicas [71, 72]. El presente trabajo contribuye a llenar ese hueco mediante la presentación de una serie de herramientas teóricas que, basándose en la consideración de la polarización del momento angular rotacional $(\mathbf{j})$ en reacciones átomo-diátomo, permiten analizar el mecanismo del proceso y determinar el control experimental obtenible mediante tal polarización.

La técnica se basa en la distinción entre momentos de polarización [25, 33] "intrínsecos" y "extrínsecos". Los primeros describen el proceso reactivo en si mismo, permiten cuantificar cómo dependen las secciones eficaces de las distribuciones espaciales correspondientes tanto al momento angular rotacional como al eje internuclear (r) y están exclusivamente determinados por la dinámica de las colisiones y no por circunstancias externas. Por su parte, los momentos de polarización extrínsecos representan la preparación de reactivos experimental: cuantifican las anisotropías de las distribuciones experimentales de $\mathbf{j}$ y $\mathbf{r}$ y, al menos en principio, pueden ser determinados por el experimentador. 
El procedimento se ilustrará en primer lugar mediante ejemplos correspondientes a la reacción

$$
\mathrm{H}+\mathrm{D}_{2}(v=0, j=2) \rightarrow \mathrm{HD}+\mathrm{D}
$$

Se demostrará que, en condiciones experimentales accesibles [97, el alineamiento de la molécula $\mathrm{D}_{2}$ tiene un notable efecto sobre los distintos observables de la reacción (distribución de estados de los productos y secciones eficaces diferencial (DCS) e integral (ICS)), de tal manera que, seleccionando la dirección del laser empleado para el bombeo Raman ("Raman pumping") estimulado, es posible controlar la eficacia de las colisiones reactivas (F.1). El análisis de dicho control nos proporcionará, además, información detallada acerca del mecanismo del proceso. En segundo lugar, se resumirán los resultados de aplicar estas técnicas al estudio del mecanismo y control de la reacción

$$
\mathrm{F}+\mathrm{H}_{2}(v=0, j=2) \rightarrow \mathrm{HF}+\mathrm{D}
$$

en un amplio intervalo de energías de colisión (incluyendo la región ultrafría ("ultracold")).

Los ejemplos se basarán tanto en cálculos de trajectorias como en cálculos mecanocuánticos independientes del tiempo, encontrandose que el acuerdo entre ambos resultados es excelente.

\section{F.2. Teoría}

\section{F.2.1. Método clásico}

Supongamos la reacción átomo-diátomo

$$
\mathrm{A}+\mathrm{BC}(v, j) \rightarrow \mathrm{AB}\left(v^{\prime}, j^{\prime}\right)+\mathrm{C}
$$

El punto de partida para la presentación clásica del método es la definición de la función de densidad de probabilidad $(\mathrm{PDF})$ clásica $P_{r}\left(\theta, \theta_{j}, \phi_{j}\right)$. Esta PDF, que es una propiedad intrínseca, representa la probabilidad de observar scattering reactivo a un cierto ángulo $\theta$ y con los productos en un estado final bien definido $\left(v^{\prime}, j^{\prime}\right)$ cuando la dirección de $\mathbf{j}$ (el momento angular rotacional de BC) está dada por los ángulos esféricos $\theta_{j}$ y $\phi_{j}$. Todas las direcciones se refieren al sistema de scattering 1 , $x y z$, en el que el eje $z$ es paralelo a la dirección de aproximación de los reactivos $(\mathbf{k})$, el plano $x z$ es el plano de scattering (el plano conteniendo a $\mathbf{k}$ y $\mathbf{k}^{\prime}$, la dirección de alejamiento

${ }^{1}$ Este sistema de referencia se denomina también sistema de centro de masa 
de los productos) y el eje $y$ es paralelo a $\mathbf{k} \times \mathbf{k}^{\prime}$. Mientras que, como se ha dicho, $\theta_{j} \mathrm{y}$ $\phi_{j}$ son el ángulo polar y azimutal que describen la dirección de $\mathbf{j}$, la posición del eje internuclear de $\mathrm{BC}(\mathbf{r})$ vendrá dada por $\theta_{r} \mathrm{y} \phi_{r}$.

La PDF clásica está normalizada

$$
\int_{-1}^{1} \int_{0}^{2 \pi} \int_{-1}^{1} P_{r}\left(\theta, \theta_{j}, \phi_{j}\right) d \cos \theta_{j} d \phi_{j} d \cos \theta=1
$$

y puede desarrollarse en serie de armónicos esféricos modificados [25, 37] según

$$
P_{r}\left(\theta, \theta_{j}, \phi_{j}\right)=\sum_{k=0}^{\infty} \sum_{q=-k}^{k} \frac{2 k+1}{4 \pi} S_{q}^{(k)}(\theta) C_{k q}^{*}\left(\theta_{j}, \phi_{j}\right)
$$

donde los coeficientes $S_{q}^{(k)}(\theta)$ (dependientes del ángulo de scattering) se denominan "polarisation dependent differential cross sections" (PDDCSs) y son los momentos de polarización intrínsecos del proceso $(\overline{F .3})$ cuando los productos se detectan a un cierto ángulo de scattering $\theta$.

Usando la ortogonalidad de los armónicos esféricos [37 para invertir el desarrollo (F.5) se llega a la siguiente expresion para las PDDCSs

$$
S_{q}^{(k)}(\theta)=\int_{0}^{2 \pi} \int_{-1}^{1} P_{r}\left(\theta, \theta_{j}, \phi_{j}\right) C_{k q}\left(\theta_{j}, \phi_{j}\right) d \cos \theta_{j} d \phi_{j}
$$

que, cuando $k=q=0$, se reduce a:

$$
S_{0}^{(0)}(\theta)=\int_{0}^{2 \pi} \int_{-1}^{1} P_{r}\left(\theta, \theta_{j}, \phi_{j}\right) d \cos \theta_{j} d \phi_{j}=P_{r}(\cos \theta)=\frac{2 \pi}{\sigma} \frac{d \sigma}{d \omega}
$$

indicando que $S_{0}^{(0)}(\theta)$ es la PDF cuyo valor representa la probabilidad de que los productos aparezcan con ángulo de scattering $\theta$, es decir, la sección eficaz diferencial normalizada.

Consideremos ahora la PDF clásica $P_{r}\left(\theta_{j}, \phi_{j}\right)$ que da la probabilidad de reacción integrada sobre el ángulo de scattering cuando el vector $\mathbf{j}$ se dispone a lo largo de la dirección dada por $\theta_{j}$ y $\phi_{j}$

$$
P_{r}\left(\theta_{j}, \phi_{j}\right)=\int_{-1}^{1} P_{r}\left(\theta, \theta_{j}, \phi_{j}\right) d \cos \theta
$$

Esta función de densidad de probabilidad puede desarrollarse también en función de 
armónicos esféricos modificados

$$
P_{r}\left(\theta_{j}, \phi_{j}\right)=\sum_{k=0}^{\infty} \sum_{q=-k}^{k} \frac{2 k+1}{4 \pi} s_{q}^{(k)} C_{k q}^{*}\left(\theta_{j}, \phi_{j}\right)
$$

donde, en este caso, los coeficientes $s_{q}^{(k)}$ del desarrollo se denominan "polarisation parameters" (PPs) y representan la polarización intrínseca de la reacción (F.3) cuando la detección de los productos no discrimina entre distintos valores del ángulo de scattering. La relación entre los PPs y las PDDCSs se deduce insertando (F.5) en (F.8) y comparando la serie resultante con (F.9)

$$
s_{q}^{(k)}=\int_{-1}^{1} S_{q}^{(k)}(\theta) d \cos \theta
$$

En concreto, para $k=q=0$ se encuentra que $s_{0}^{(0)}=1$.

Los PDDCSs y los PPs son las cantidades claves a la hora de (i) cuantificar la dependencia de las secciones eficaces con la polarización experimental (extrínseca) de los reactivos y (ii) analizar el mecanismo por el que trancurren las colisiones. $\mathrm{Al}$ igual que los orbitales atómicos, tanto las PDDCSs como los PPs pueden expresarse como cantidades complejas y reales [25, 50]: mientras que la primera representación (cantidades complejas) facilita el manejo de expresiones que involucran a los momentos intrínsecos, la segunda (cantidades reales) permite interpretarlos en función de las coordenadas cartesianas del espacio. La notación empleada será $S_{q}^{(k)}(\theta)\left(s_{q}^{(k)}\right)$ para los momentos complejos y $S_{q}^{\{k\}}(\theta)\left(s_{q}^{\{k\}}\right)$ para los momentos reales. La transformación entre ambos conjuntos de PDDCSs viene dada por las ecuaciones

$$
\begin{aligned}
& S_{q+}^{\{k\}}(\theta)=\frac{1}{\sqrt{2}}\left[(-1)^{q} S_{q}^{(k)}(\theta)+S_{-q}^{(k)}(\theta)\right] \quad 1 \leq q \leq k \\
& S_{0}^{\{k\}}(\theta)=S_{0}^{(k)}(\theta) \\
& S_{q-}^{\{k\}}(\theta)=\frac{1}{i \sqrt{2}}\left[(-1)^{q} S_{q}^{(k)}(\theta)-S_{-q}^{(k)}(\theta)\right] \quad 1 \leq q \leq k
\end{aligned}
$$

que son igualmente válidas para PPs.

A la hora de describir un experimento y, en concreto, la polarización extrínseca que lo caracteriza, es necesario considerar un segundo sistema de referencia: el sistema de laboratorio XYZ, cuyo origen coincide con el del sistema de centro de masa ( $x y z)$ y cuya orientación espacial está fija. Los ángulos de Euler $\alpha, \beta$ y $\gamma$ representarán la rotación necesaria para llevar al sistema de centro de masa hasta el sistema de laboratorio. Se utilizarán las letra mayúsculas $Q, \Theta_{j}$ y $\Phi_{j}$ para indicar las componentes 


\section{Scattering frame}

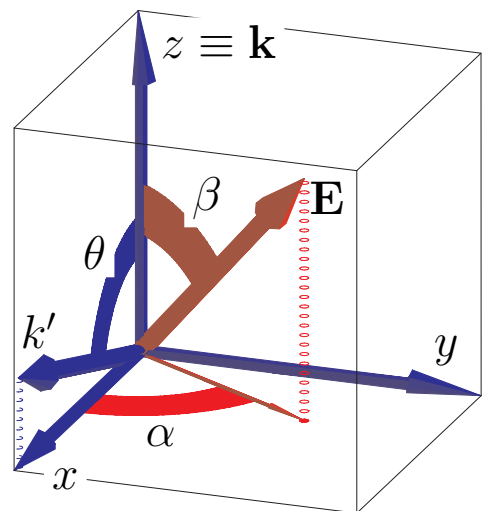

Laboratory frame

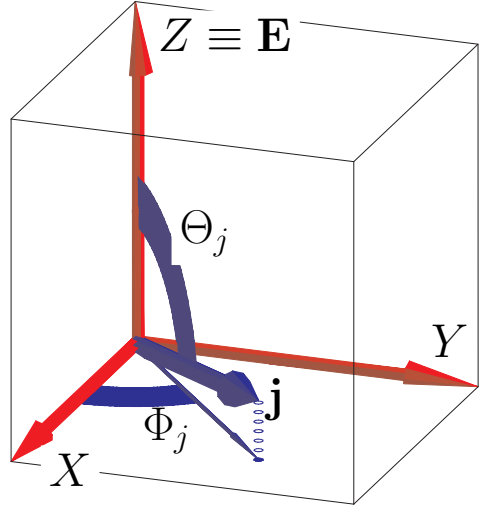

Fig. F.1.: Sistemas de scattering ( $x y z)$ y de laboratorio $(X Y Z)$.

de los momentos y la posición de $\mathbf{j}$ definidos en el sistema de laboratorio. La Fig. F.1 sirve como ilustración del significado de estos ángulos y de la relación entre ambos sistemas de referencia (el eje $Z$ del sistema de laboratorio se representa como E por razones que quedarán claras más adelante).

Si los reactivos se preparasen en el laboratorio según los momentos $A_{Q}^{(k)}$, los correspondientes momentos extrínsecos en el sistema de centro de masa $\left(a_{q}^{(k)}\right)$ vendrían dados por la expresión

$$
a_{q}^{(k)}=\sum_{Q=-k}^{k} D_{q Q}^{k *}(\alpha, \beta, \gamma) A_{Q}^{(k)}
$$

donde $D_{q Q}^{k}(\alpha, \beta, \gamma)$ representa una matriz de rotación.

Una vez que sabemos cómo describir la polarización intrínseca y la polarización extrínseca, estamos en condiciones de abordar el problema del control de la reacción mediante la obtención de expresiones para los diferentes observables en función de la polarización extrínseca de los reactivos. A tal fin, la sección eficaz diferencial (DCS) se escribirá

$$
\frac{d \sigma}{d \omega}=\frac{\sigma_{i s o}}{4 \pi} \int_{0}^{2 \pi} \int_{-1}^{1} 4 \pi P\left(\theta, \theta_{j}, \phi_{j}\right) \rho\left(\theta_{j}, \phi_{j}\right) d \cos \theta d \phi
$$

donde $\sigma_{i s o}$ es la sección eficaz integral de la reacción cuando los reactivos no están polarizados, $\rho\left(\theta_{j}, \phi_{j}\right)$ es la PDF que describe la polarización extrínseca de los reactivos y el factor $4 \pi$ dentro de la integral asegura su correcta normalización.

La PDF extrínseca $\rho\left(\theta_{j}, \phi_{j}\right)$ puede expandirse según una serie multipolar totalmente 
similar a (F.5), dependiente en este caso de los momentos de polarización extrínsecos $a_{q}^{(k)}$. Introduciendo las expansiones de $P\left(\theta, \theta_{j}, \phi_{j}\right)$ y $\rho\left(\theta_{j}, \phi_{j}\right)$ en la expresión para la DCS (F.13) y usando la ortogonalidad de los armónicos esféricos modificados [37, se llega a la ecuación

$$
\frac{d \sigma}{d \omega}=\frac{\sigma_{\text {iso }}}{2 \pi} \sum_{k=0}^{\infty} \sum_{q=-k}^{k}(2 k+1) S_{q}^{(k) *}(\theta) a_{q}^{(k)}
$$

que permite evaluar la DCS para cualquier polarización experimental de los reactivos.

Una expresión equivalente para la sección eficaz integral (ICS) se obtiene integrando (F.14) a todas las direcciones del espacio

$$
\sigma=\sigma_{\text {iso }} \sum_{k=0}^{\infty}(2 k+1) s_{0}^{(k)} a_{0}^{(k)}
$$

La mayor diferencia entre esta ecuación y (F.14) reside en que, mientras la DCS depende de todos los PDDCSs y de todos los momentos de polarización extrínsecos, la ICS depende exclusivamente de los momentos con $q=0$. Esta diferencia proviene de que la información contenida en los momentos con $q \neq 0$ impone de manera implícita que se conoce la posición del plano de scattering. Sin embargo, al evaluar la ICS integramos a todas las direcciones del espacio y, en particular, a todas las posibles posiciones de dicho plano. Despues de tal integración no tiene sentido hablar del plano de scattering y la ICS no puede depender de momentos cuya información está referida a su posición.

\section{F.2.2. Método mecano-cuántico}

El punto de partida para obtener una expresión mecano-cuántica de los momentos intrínsecos (PDDCSs y PPs) son los elementos de la matriz de scattering en la representación de helicidad [73, la representación mejor adaptada al estudio de las correlaciones $\mathbf{k}-\mathbf{k}^{\prime} \mathbf{- j}$. Al ser dichos elementos independientes de $M$ (la proyección del momento angular total $\mathbf{J}$ ) y como las ecuaciones que emplearemos sólo requieren implícitamente el uso de la energía total y de los números cuánticos que describen la configuración atómica y el nivel vibracional de las moléculas, representaremos los elementos de la matriz de scattering en la representación de helicidad mediante $S_{j^{\prime} \Omega^{\prime}, j \Omega}^{J}$ $\left(j\left(j^{\prime}\right)\right.$ es el número cuántico rotacional para la molécula diatómica reactiva (producto), $\Omega\left(\Omega^{\prime}\right)$ es el número cuántico de helicidad de los reactivos (productos) y $J$ es el número cuántico para el momento angular total). 
Usando esta notación, la amplitud de scattering puede escribirse como 74

$$
f_{\Omega^{\prime} \Omega}(\theta)=\frac{1}{2 i k_{i n}} \sum_{J}(2 J+1) d_{\Omega^{\prime} \Omega}^{J}(\theta) S_{j^{\prime} \Omega^{\prime}, j \Omega}^{J}
$$

siendo $d_{\Omega^{\prime} \Omega}^{J}(\theta)$ una matriz de rotación reducida y $k_{i n}$ el número de onda asociado a los reactivos. Las PDDCSs intrínsecas vendrán dadas por

$$
\left[S_{q}^{(k)}(\theta)\right]^{*}=\sum_{\Omega^{\prime} \Omega_{1} \Omega_{2}} \bar{f}_{\Omega^{\prime} \Omega_{1}}(\theta) \bar{f}_{\Omega^{\prime} \Omega_{2}}^{*}(\theta)\left\langle j \Omega_{1}, k q \mid j \Omega_{2}\right\rangle,
$$

donde $\bar{f}_{\Omega^{\prime} \Omega}(\theta)$ representa

$$
\bar{f}_{\Omega^{\prime} \Omega}(\theta)=\frac{\sum_{J}(2 J+1) d_{\Omega^{\prime} \Omega}^{J}(\theta) S_{j^{\prime} \Omega^{\prime}, j \Omega}^{J}}{\left[2 \sum_{J \Omega^{\prime} \Omega}(2 J+1)\left|S_{j^{\prime} \Omega^{\prime}, j \Omega}^{J}\right|^{2}\right]^{1 / 2}} .
$$

La DCS para una polarización arbitraria de los reactivos viene dada por 24

$$
\frac{d \sigma}{d \omega}=\sum_{\Omega^{\prime} \Omega_{1} \Omega_{2}} f_{\Omega^{\prime} \Omega_{1}}(\theta) f_{\Omega^{\prime} \Omega_{2}}^{*}(\theta)\left\langle j \Omega_{1}|\hat{\rho}| j \Omega_{2}\right\rangle
$$

donde $\left\langle j \Omega_{1}|\hat{\rho}| j \Omega_{2}\right\rangle$ es un elemento de la matriz de densidad de los reactivos (como se impone que los productos están en un estado rotacional bien definido, el operador densidad $\hat{\rho}$ sólo considera las coherencias entre differentes helicidades) que describe la preparación experimental de los mismos. Estos elementos de matriz se relacionan con los momentos de polarización extrínsecos según [25, 33.

$$
\left\langle j \Omega_{1}|\hat{\rho}| j \Omega_{2}\right\rangle=\sum_{k=0}^{2 j} \sum_{q=-k}^{k} \frac{2 k+1}{2 j+1} a_{q}^{(k)}\left\langle j \Omega_{1}, k q \mid j \Omega_{2}\right\rangle
$$

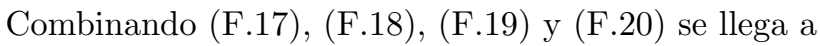

$$
\frac{d \sigma}{d \omega}=\frac{\sigma_{\text {iso }}}{2 \pi} \sum_{k=0}^{2 k} \sum_{q=-k}^{k}(2 k+1) S_{q}^{(k) *}(\theta) a_{q}^{(k)}
$$

que es idéntica a la expresión (F.14) encontrada en la sección anterior excepto por el hecho de que la suma en $k$ está limitada a valores que van entre 0 y $2 j$ (consecuencia de la simetría de los coeficientes de Clebsch-Gordan). Integrando sobre todas las 
direcciones del espacio llegaríamos a

$$
\sigma=\sigma_{\text {iso }} \sum_{k=0}^{2 k}(2 k+1) s_{0}^{(k)} a_{0}^{(k)}
$$

para la ICS.

La interpretación de los momentos intrínsecos y extrínsecos es idéntica independientemente de si trabajamos usando mecánica clásica o cuántica. En la práctica, las series infinitas clásicas se pueden truncar en $k=2 j$ transformándose los resultados en resultados cuasiclásicos.

\section{F.2.3. Retratos estereodinámicos}

Hasta ahora la exposición se ha centrado en el empleo de los momentos de polarización intrínsecos para determinar el control de los observables de reacción que puede lograrse mediante una adecuada polarización experimental de los reactivos.

Sin embargo, su utilidad va mucho más allá. Los momentos intrínsecos representan la polarización de los reactivos cuando la reacción tiene lugar o, en otras palabras, la polarización de los reactivos que da lugar a la formación de productos. Por lo tanto, mediante su análisis es posible caracterizar el mecanismo (o mecanismos) de la reacción.

La manera más conveniente de llevar a cabo tal estudio es mediante los retratos estereodinámicos ("stereodynamical portraits"): figuras tridimensionales que combinan la información contenida en un determinado conjunto de momentos de polarización y proporcionan una visión inmediata de las distribuciónes de $\mathbf{r}$ y $\mathbf{j}$ asociadas a esos momentos. Las expresiones de estas distribuciones son [40]

$$
P_{r}\left(\theta_{r}, \phi_{r}\right)=\sum_{k=0}^{2 j} \sum_{q=-k}^{k} \frac{2 k+1}{4 \pi} s_{q}^{(k)}\langle j 0, k 0 \mid j 0\rangle C_{k q}^{*}\left(\theta_{r}, \phi_{r}\right)
$$

para los retratos asociados al eje internuclear ("internuclear axis potraits") y

$$
Q_{r}\left(\theta_{j}, \phi_{j}\right)=\sum_{k=0}^{2 j} \sum_{q=-k}^{k} \frac{2 k+1}{4 \pi} s_{q}^{(k)}\langle j j, k 0 \mid j j\rangle C_{k q}^{*}\left(\theta_{j}, \phi_{j}\right)
$$

para los retratos asociados al momento angular rotacional ("rotational portraits").

En estas expresiones se han utilizado los PPs $\left(s_{q}^{(k)}\right)$ para generar los retratos. Sin 
embargo, sería posible haber empleado las PDDCSs o los momentos extrínsecos. Lo único que cambiaría sería el significado físico de las distribuciones tridimensionales obtenidas: (i) las distribuciones asociadas a los PPs representan la distribución de $\mathbf{r}$ y j que da lugar a la reacción (F.3), (ii) las asociadas a las PDDCSs son la distribución de $\mathbf{r}$ y $\mathbf{j}$ que da lugar a la reacción (F.3) a un ángulo de scattering determinado y (iii) las asociadas a los momentos extrínsecos representan las distribuciones experimentales de $\mathbf{r}$ y $\mathbf{j}$.

\section{F.3. Ejemplos y resultados}

\section{F.3.1. Determinación experimental de la influencia de la polarización de los reactivos}

Describiremos, en primer lugar, un experimento de haces moleculares que podría usarse para determinar la validez de las prediciones teóricas llevadas a cabo mediante las herramientas introducidas en la Sección anterior.

El experimento es, en principio, capaz de polarizar cualquier molécula supceptible de sufrir excitación Raman estimulada. Nuestra presentación se centrará, sin embargo, en la reacción

$$
\mathrm{H}+\mathrm{D}_{2}(v=0, j=2) \rightarrow \mathrm{HD}\left(v^{\prime}, j^{\prime}\right)+\mathrm{D}
$$

El experimento consiste en alinear el momento angular rotacional (j) de la molécula $\mathrm{D}_{2}$ mediante la preparación de ésta en el estado $|v=0, j=2, m=0\rangle$, donde la proyección de momento angular $m$ se refiere al eje $Z$ del sistema de laboratorio. La preparación de la molécula reactivo en este estado puede conseguirse mediante Raman estimulado seleccionando las frecuencias "pump" y "Stokes" de manera adecuada [97. De esta manera, el eje $Z$ del sistema de laboratorio coincidiría con la dirección del laser responsable de la transición ${ }^{2}$ y el eje internuclear de la molécula $\mathrm{D}_{2}(v=0, j=2)$ quedaría dispuesto paralela/antiparalelamente a la dirección $Z$, mientras que j se colocaría perpendicular a la misma. Cambiando la dirección del eje $Z$, se cambia la preparación experimental de $\mathbf{j}$ y $\mathbf{r}$ con respecto al eje $\mathbf{k}$ y, si el experimento incluye detección de productos resuelta en ángulo de scattering, también con respecto al plano de scattering (plano $\mathbf{k}-\mathbf{k}^{\prime}$ ).

Para un estado puro $|j=2, m=0\rangle$, la polarización de $\mathbf{r}$ y $\mathbf{j}$ en el sistema de laboratorio está completamente determinada por tres momentos de polarización $A_{Q}^{(k)}$, cuyos

\footnotetext{
${ }^{2}$ Por esta razón, en la Fig F.1 el eje $Z$ del sistema de laboratorio se representó como E
} 

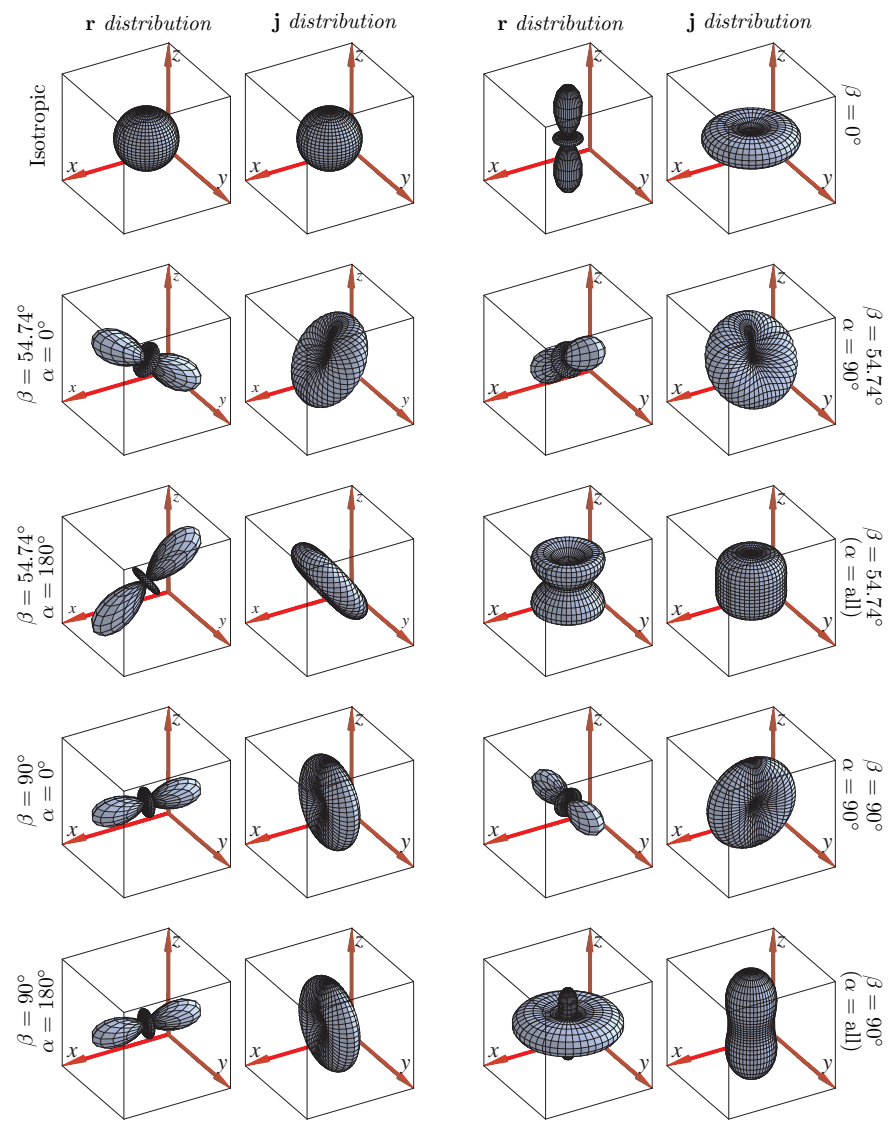

Fig. F.2.: Distribuciones experimentales de $\mathbf{r}$ y $\mathbf{j}$ correspondientes a los reactivos sin polarizar ("isotropic") y a diferentes preparaciones de ejes (incluyendo los valores de $\alpha$ y $\beta$ empleados en cada caso). Todas las figuras se refieren al sistema de centro de masa $x y z$.

valores mecano-cuánticos son:

$$
A_{0}^{(0)}=0 \quad A_{0}^{(2)}=-\sqrt{\frac{2}{7}} \quad A_{0}^{(4)}=\sqrt{\frac{2}{7}}
$$

Como la preparación de ejes tiene simetría axial (en el sistema de laboratorio), únicamente los momentos con $Q=0$ pueden ser distintos de cero.

Los valores de los momentos extrínsecos en el sistema de centro de masa $x y z$ pueden obtenerse mediante el uso de la expresión (F.12), llevándonos a

$$
a_{q}^{(k)}=D_{q 0}^{k *}(\alpha, \beta, \gamma) A_{0}^{(k)}=C_{k q}(\beta, \alpha) A_{0}^{(k)}
$$


Dado que las preparaciones de ejes tienen simetría axial en el sistema $X Y Z$, los únicos ángulos de Euler que se requieren son $\alpha$ y $\beta$, los ángulos azimutal y polar que especifican la posición de $Z$ en el sistema de centro de masa (ver la Fig.F.1, en la que se ha identificado el eje $Z$ con la dirección del laser E).

Varios ejemplos de las preparaciones de ejes que se pueden obtener mediante este experimento se presentan en la Fig. (F.2) donde, despues de evaluar los momentos extrínsecos correspondientes a cada preparación de ejes mediante (F.27), se han empleado los retratos estereodinámicos para obtener las distribuciones de $\mathbf{r}$ y $\mathbf{j}$ asociadas a esos momentos.

\section{F.3.2. Reacción $\mathbf{D}+\mathbf{H}_{2}(v=0, j=2)$}

\section{Control}

La naturaleza y magnitud del control de la reacción (F.25) mediante el experimento anteriormente propuesto se discutirá a continuación. Los resultados se basan en cálculos mecano-cuánticos [112 y de trayectorias cuasiclásicas 25] sobre la superficie BKMP2 [111].

En el experimento, la dirección de alineamiento de $\mathbf{r}$ en el sistema de centro de masa está determinado por los ángulos $\alpha$ y $\beta$. En particular, nosotros consideraremos $\beta=0,54.74$ y $90^{\circ}\left(54.74^{\circ}\right.$ es el ángulo mágico) y $\alpha=0,45$ y $180^{\circ}$. Es fundamental a la hora de interpretar los resultados tener claro que, mientras el eje internuclear $\mathbf{r}$ está alineado a lo largo de la dirección definida por $\alpha$ y $\beta$, el momento angular rotacional se encuentra situado perpendicular a tal dirección. Por lo tanto, cuando $\beta=0^{\circ}$ el eje internuclear será paralelo a $\mathbf{k}$ y las colisiones serán colineales ("headon") y cuando $\beta=90^{\circ}$ el eje internuclear será perpendicular a $\mathbf{k}$ y las colisiones serán laterales ("side-on").

La Fig. F.3 representa la función de excitación (la sección eficaz integral sumada sobre estados finales) para la reacción (F.25) en función de la energía de collisión. La línea continua corresponde al caso más habitual, reactivos sin polarizar, mientras que las demás incluyen el efecto del alineamiento de $\mathrm{D}_{2}$ y nos permiten concluir que: la reactividad es favorecida por colisiones colineales $\left(\beta=0^{\circ}\right)$, dificultada por colisiones laterales $\left(\beta=90^{\circ}\right)$ y apenas alterada por la preparación correspondiente al ángulo mágico. Aunque este no es un resultado inesperado (se sabe que la reacción $\mathrm{D}+\mathrm{H}_{2}$ es "collinearly constrained"), la Fig. F.3 cuantifica este efecto y establece en que medida se puede controlar la reactividad seleccionando la polarización adecuada.

El efecto del alineamiento de los reactivos sobre la distribución rotacional de la molécula HD cuando $v^{\prime}=0$ y $E_{\text {coll }}=1.306 \mathrm{eV}$ se ilustra en la Fig. F.4, que contiene 


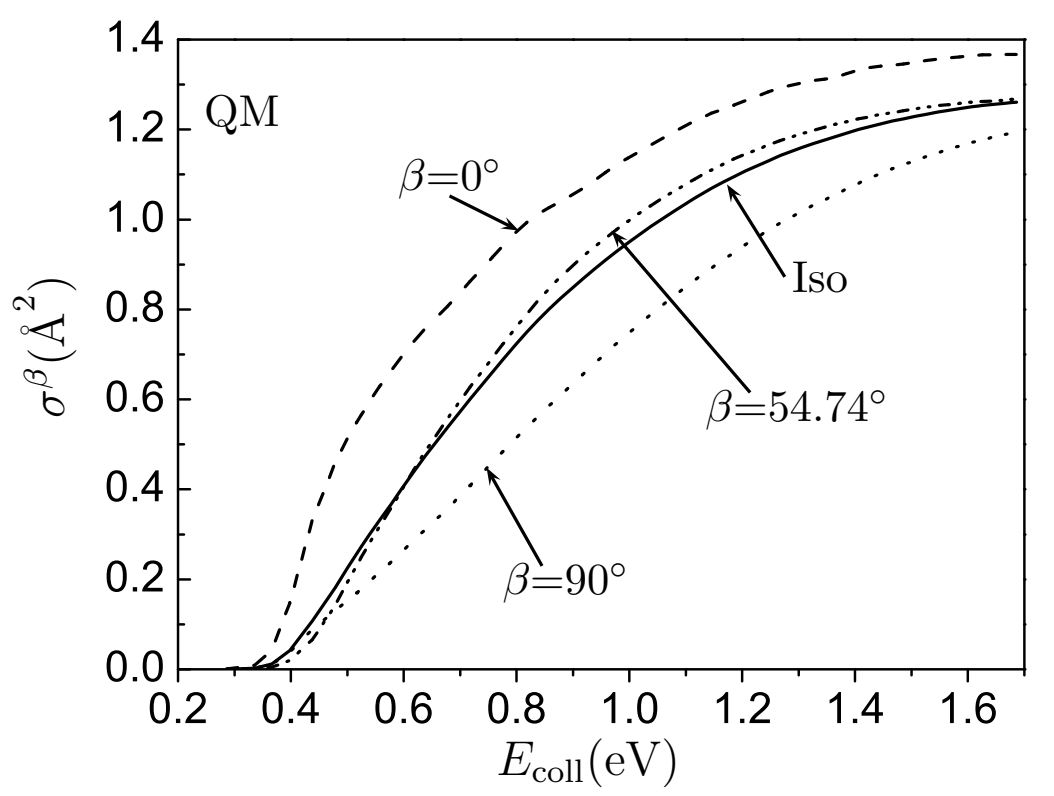

Fig. F.3.: Función de excitación mecano-cuántica para la reacción $\mathrm{H}+\mathrm{D}_{2}(v=0, j=2)$ y diferentes alineamientos de la molécula $\mathrm{D}_{2}$. La curva indicada como "iso" corresponde a los reactivos sin polarizar, es decir, aquella situación en que las distribuciones de los vectores $\mathbf{j}$ y $\mathbf{r}$ antes de la colisión son isotrópicas, de tal manera que dichos vectores no se alinean $u$ orientan a lo largo de ninguna dirección en el espacio.

tanto resultados cuasiclásicos (panel superior) como mecano-cuánticos (panel inferior). Ambos tipos de resultados muestran un notable acuerdo, encontrándose en los dos casos que: las colisiones colineales $\left(\beta=0^{\circ}\right)$ hacen la distribución rotacional más fría, las colisiones laterales $\left(\beta=90^{\circ}\right)$ la hacen más caliente y las colisiones caracterizadas por el ángulo mágico no alteran la forma de la distribución isotrópica. Es interesante resaltar que las ICSs correspondientes a la formación de productos en los estados rotacionales $j^{\prime}=0-6$ y $j^{\prime}=8-12$ responden al alineamiento de los reactivos de manera casi opuesta.

Únicamente los parámetros de polarización $s_{0}^{(0)}, s_{0}^{(2)}$ y $s_{0}^{(4)}$ contribuyen a la sección eficaz integral (ver $(\overline{\mathrm{F} .22})$ ). De estos, $s_{0}^{(2)}$ es el principal responsable de los cambios en el valor del observable. Este momento es bastante negativo (positivo) para $j^{\prime}<5\left(j^{\prime}>\right.$ 10) y cambia de signo cuando $j^{\prime}=8$. Estos valores explican porqué preparaciones de los ejes con $a_{0}^{(2)}=-0.535,0$ ó 0.237 (estos són los valores mecano-cuánticos del momento de polarización extrínseco cuando $\beta$ toma los valores $0,54.74$ o $90^{\circ}$ respectivamente) llevan a distribuciones rotacionales de los productos que van siendo progresivamente 

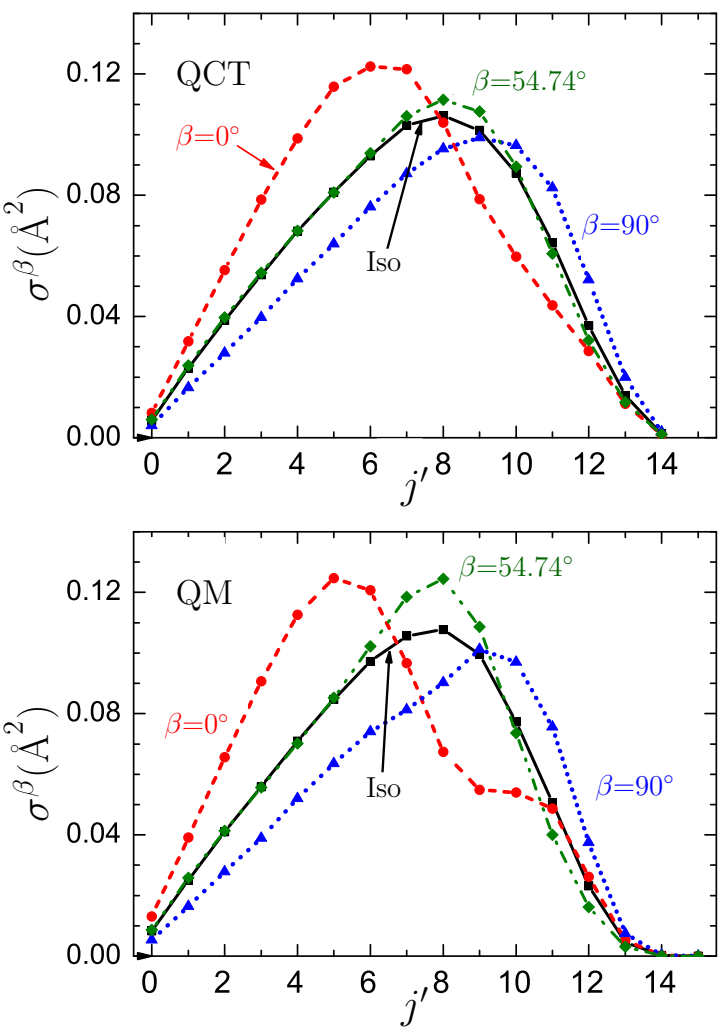

Fig. F.4.: Distribución rotacional de los productos de la reacción $\mathrm{H}+\mathrm{D}_{2}(v=0, j=2) \rightarrow \mathrm{HD}\left(v^{\prime}=0, j^{\prime}\right)+\mathrm{D} \quad$ a $\quad E_{\text {coll }}=1.306 \quad \mathrm{eV}$. Las distintas curvas corresponden a los reactivos sin polarizar (iso) y a diferentes alineamientos de la molécula $\mathrm{D}_{2}$. El panel superior corresponde a resultados cuasiclásicos y el inferior a resultados mecano-cuánticos.

más calientes.

El análisis del efecto de la polarización de los reactivos sobre la sección eficaz diferencial requiere considerar el ángulo azimutal $\alpha$ además del ángulo $\beta$. Como se demuestra a continuación, esto incrementa la estereoespecificidad del experimento y permite un control de la reacción más "fino" que el obtenido para las secciones integrales.

La Fig. F.5 contiene la DCS sumada sobre niveles rotacionales de la reacción (F.25) cuando $v^{\prime}=0$ y $E_{\text {coll }}=1.306 \mathrm{eV}$. El panel superior incluye resultados cuasiclásicos y el inferior mecano-cuánticos. Un análisis rápido de los resultados permite concluir dos cosas: (i) el acuerdo entre ambos tipos de resultados es muy notable (tanto más cuanto que la consideración de la DCS implica un gran nivel de detalle en el estudio 

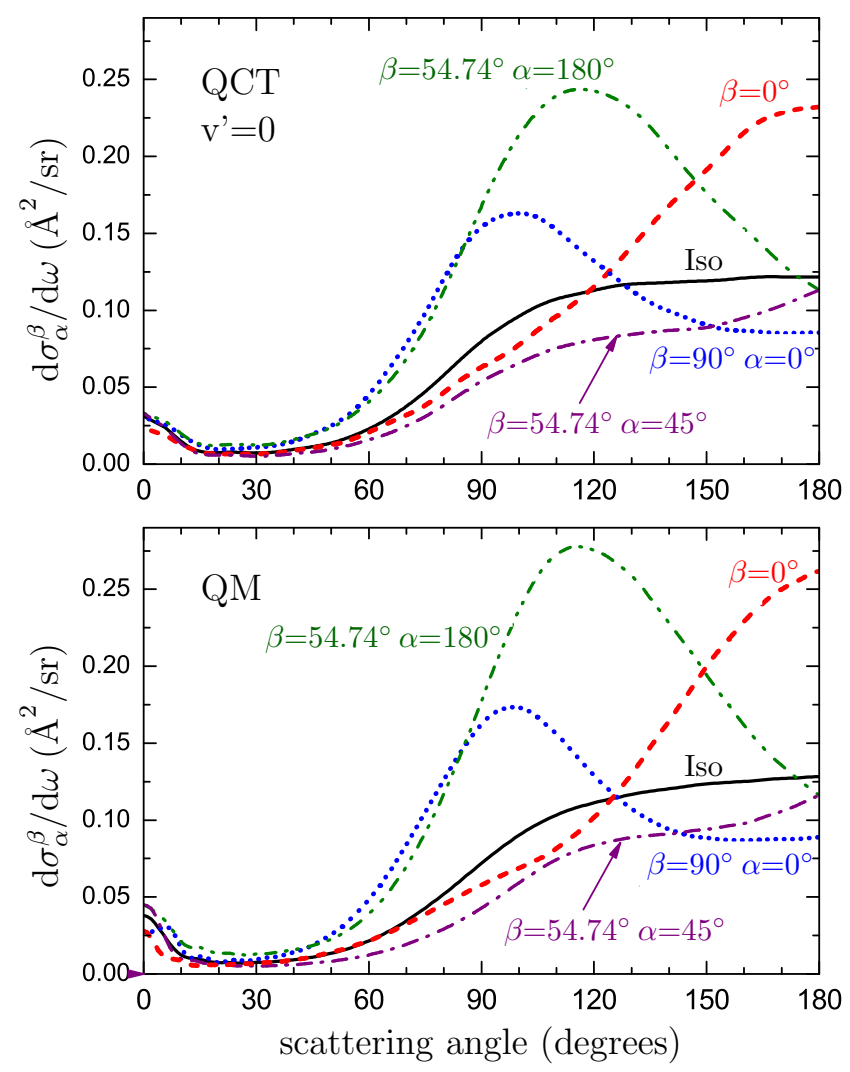

Fig. F.5.: Sección eficaz diferencial de la reacción $\mathrm{H}+\mathrm{D}_{2}(v=0, j=2) \rightarrow \mathrm{HD}\left(v^{\prime}=0, j^{\prime}\right)+\mathrm{D}$ sumada sobre los niveles rotacionales finales cuando $E_{\text {coll }}=1.306 \mathrm{eV}$. Las distintas curvas corresponden a los reactivos sin polarizar (iso) y a diferentes alineamientos de la molécula $\mathrm{D}_{2}$. El panel superior corresponde a resultados cuasiclásicos y el inferior a resultados mecano-cuánticos.

de la reacción) y (ii) cambios en la preparación de los reactivos provocan drásticas modificaciones de la forma de la DCS.

Las colisiones colineales $\left(\beta=0^{\circ}\right)$ dan lugar a una distribución angular que, comparada con la obtenida en el caso isotrópico, favorece el scattering en la dirección "backward" $\left(\theta=180^{\circ}\right)$. A su vez, las colisiones laterales $\left(\beta=90^{\circ}\right)$ aumentan el scattering "sideways". El alineamiento correspondiente al ángulo mágico lleva a una situación intermedia entre lo observado para los dos casos anteriores.

Es de destacar, por último, la influencia del ángulo azimutal $\alpha$, cuyo valor puede tener un efecto espectacular sobre la forma de la DCS. Valgan como ejemplo los resultados correspondientes a $\beta=54.74^{\circ}, \alpha=45^{\circ}$ y a $\beta=54.74^{\circ}, \alpha=180^{\circ}$ : mientras que en 
el primer caso el alineamiento de la molécula $\mathrm{D}_{2}$ lleva a una DCS de forma similar a la isotrópica, aunque mucho menos intensa, la segunda preparación de ejes da lugar a un notable incremento de la DCS, que es especialmente pronunciado alrededor de $\theta=115^{\circ}$. Los valores $\alpha=0$ y $180^{\circ}$ corresponden a preparaciones de los reactivos donde el eje internuclear de la diatómica está sobre (o cerca de) el plano de scattering. Por el contrario, valores del ángulo azimutal comprendidos en el intervalo $45^{\circ} \leq \alpha \leq 135^{\circ}$ indican que predominan las geometrías donde el eje internuclear está aproximadamente perpendicular al plano de scattering. El hecho que se obtenga un gran incremento de la DCS cuando $\alpha=180^{\circ}$ sugiere que la reacción es predominantemente colineal, es decir, que el plano formado por los tres átomos permanece constante durante el transcurso de la reacción y coincide con el plano de scattering.

\section{Mecanismo}

Además de sobre el control de la reacción (F.25), las páginas anteriores nos proporcionaron pistas acerca del mecanismo de dichas colisiones. Sin embargo, para llevar a cabo un estudio pormenorizado del mecanismo es más conveniente utilizar directamente los momentos de polarización intrínsecos en combinación con los retratos estereodinámicos.

Dicho análisis permite caracterizar dos mecanismos distintos para las colisiones $\mathrm{D}+\mathrm{H}_{2}$ que, manteniendo la nomenclatura utilizada por otros autores [110, llamaremos directo ("direct") y retrasado ("delayed").

El primero es un mecanismo directo, responsable de la mayor parte de la reactividad (en concreto, de todo el scattering "backward" y "sideways") y asociado a valores del número cuántico de momento angular total $(J)$ medios y bajos. La estereodinámica del mecanismo viene fundamentalmente determinada por dos momentos intrínsecos reales: $S_{1-}^{\{1\}}(\theta)$ y $S_{0}^{\{2\}}(\theta)$ (y sus correspondientes PPs cuando se consideran los resultados integrados sobre el ángulo de scattering). El primero determina la orientación de $\mathbf{j}$ con respecto al eje $y$ del sistema de centro de masa y, el segundo, la inclinación de $\mathbf{r}$ respecto al eje $z \equiv \mathbf{k}$, la dirección de aproximación de los reactivos.

Un examen detallado de los valores $\frac{3}{3}$ de $S_{1-}^{\{1\}}(\theta)$ y $s_{1-}^{\{1\}}$ permite concluir que existe una relación entre éstos y la excitación rotacional de los productos. Tal relación se explica mediante la Fig. F.6: si j está orientado de modo paralelo (antiparalelo) al eje $y$, los átomos $\mathrm{H}$ y D chocan mientras se mueven a lo largo de direcciones aproximadamente opuestas (similares), formándose un complejo H-D-D con gran (poca) excitación "bending" que, al romperse, da lugar a la formación de productos rotacionalmente

${ }^{3}$ Valores positivos (negativos) de los momentos $S_{1-}^{\{1\}}(\theta)$ y $s_{1-}^{\{1\}}$ indican que $\mathbf{j}$ se orienta paralelamente (antiparalelamente) a $y$ 


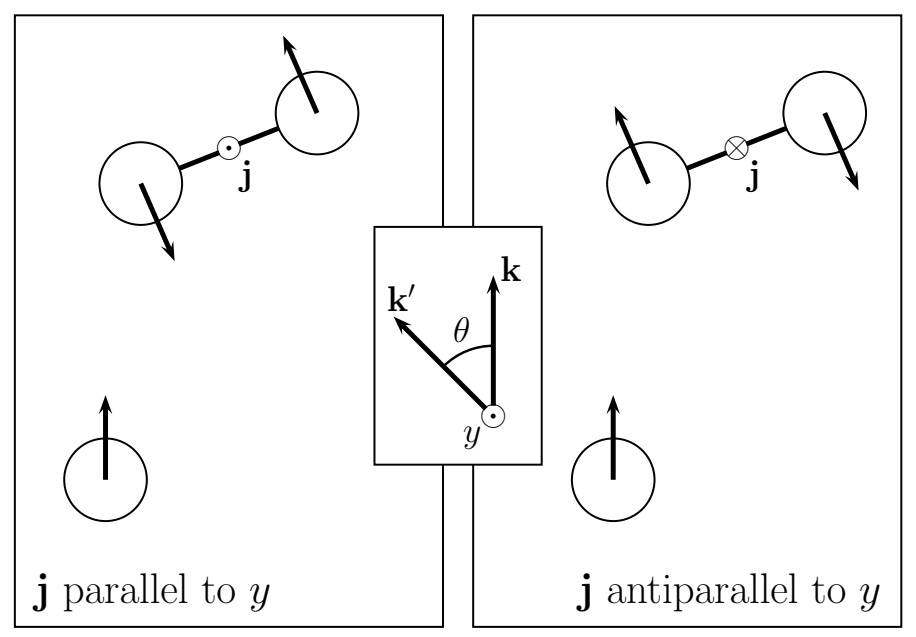

Fig. F.6.: Ilustración que ejemplifica la influencia de la orientacion de la molécula $\mathrm{D}_{2}$ sobre la excitación rotacional de los productos, es decir, de la molécula HD. Si la orientación de $\mathbf{j}$ es paralela (antiparalela) a $y \equiv \mathbf{k} \times \mathbf{k}^{\prime}$, los átomos H y $\mathrm{D}$ que colisionan lo hacen moviéndose sobre direcciones aproximadamente opuestas (similares). Esto llevará a la formación de un complejo H-D-D con más (menos) energía vibracional "bending" que, a su vez, da lugar a más (menos) excitación rotacional de los productos.

muy (poco) excitados. Como ilustración del esta característica de la reacción, la Fig. F.7 presenta la distribución rotacional y los retratos estereodinámicos intrínsecos correspondientes a la reacción $\mathrm{H}+\mathrm{D}_{2}(v=0, j=2) \rightarrow \mathrm{HD}\left(v^{\prime}=0, j^{\prime}\right)+\mathrm{D}$ cuando $E_{\text {coll }}=1.506$ eV. Para facilitar la visualización de los retratos, el origen del sistema de centro de masa se ha desplazado del centro del cubo (su posición real) a una esquina posterior del mismo. Los retratos asociados al momento angular rotacional de $\mathrm{D}_{2}$ (fila central) representan las distribuciónes de $\mathbf{j}$ que dan lugar a la formación de los productos para cada uno de los valores de $j^{\prime}$ y muestran de una manera clara como los valores bajos (altos) de $j^{\prime}$ correlacionan con colisiones donde $\mathbf{j}$ está orientado a lo largo de $-y(+y)$.

El valor de los momentos $S_{0}^{\{2\}}(\theta)$ y $s_{0}^{\{2\}}$ se relaciona con el alineamiento de $\mathbf{j}$ y $\mathbf{r}$ con respecto a k. Valores positivos (negativos) de dichos momentos corresponden (i) a $\mathbf{j}$ alineado paralelamente (perpendicularmente) a $\mathbf{k}$ y a (ii) $\mathbf{r}$ dispuesto perpendicularmente (paralelamente) a k. Siempre que el mecanismo directo sea el mecanismo dominante (lo cual, como se discutirá más tarde, es verdad siempre excepto cuando consideremos reacciones estado-a-estado en la zona "forward"), se encuentra que los valores de los momentos $S_{0}^{\{2\}}(\theta)$ y $s_{0}^{\{2\}}$ vienen determinados por la necesidad de facilitar a los reactivos el acceso a la zona del estado de transición, que para esta reacción 


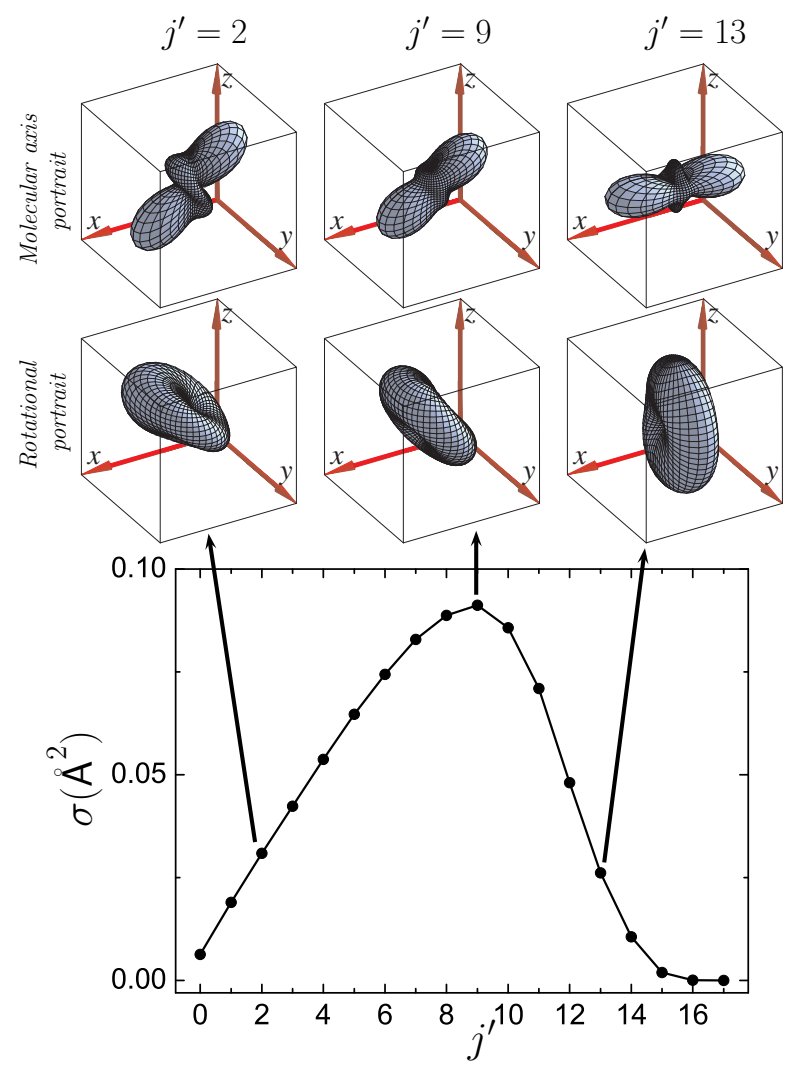

Fig. F.7.: Distribución rotacional mecano-cuántica de los productos de la reacción $\mathrm{H}+\mathrm{D}_{2}(v=0, j=2) \rightarrow \mathrm{HD}\left(v^{\prime}=0, j^{\prime}\right)+\mathrm{D}$ a $E_{\text {coll }}=1.506$ eV y retratos estereodinámicos intrínsecos correspondientes al eje internuclear de la molécula $\mathrm{D}_{2}$ (fila superior) y a su momento angular rotacional (fila intermedia).

es lineal y muy rígido (ligeras desviaciones de la geometría lineal llevan a notables incrementos de energía). Esto significa que, cuando predominen los parámetros de impacto bajos, dichos valores mostrarán que $\mathbf{r}$ tiende a disponerse a lo largo de $\mathbf{k}$ mientras que, por el contrario, cuando dominen los parámetros de impacto altos, los valores de los momentos indicarán que $\mathbf{r}$ se "tumba" y se coloca perpendicularmente a k. Un ejemplo de este comportamiento se encuentra en los retratos estereodinámicos para $\mathbf{r}$ incluídos en la Fig. F.7 (fila superior): a medida que aumenta la excitación rotacional de los productos los parámetros de impacto que participan en la reacción van siendo mayores y, en consecuencia, el eje internuclear se tumba con respecto a $\mathbf{k}$.

El segundo de los mecanismos identificado en relación con el proceso F.25 (el mecanismo retrasado) es responsable de los picos "forward" que aparecen en las DCSs 

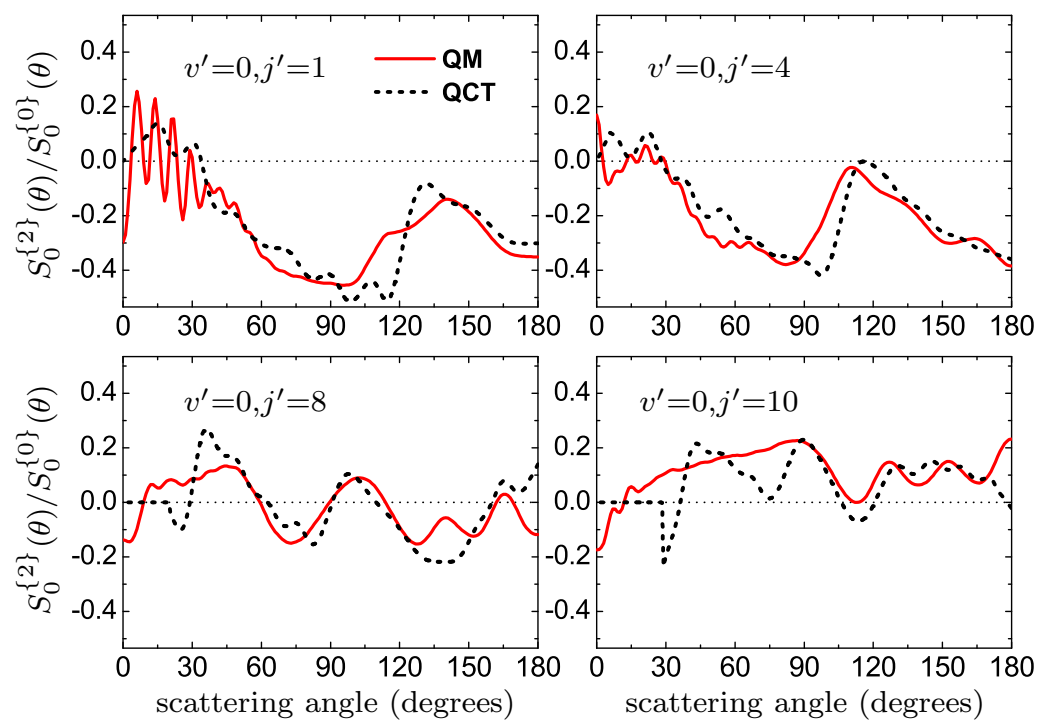

Fig. F.8.: $S_{0}^{\{2\}}(\theta)$ renormalizada para la $\mathrm{H}+\mathrm{D}_{2}(v=0, j=2) \rightarrow \mathrm{HD}\left(v^{\prime}=0, j^{\prime}=1,4,8\right.$ y $10)+\mathrm{D}$ a $E_{\text {coll }}=1.306 \mathrm{eV}$. Las líneas continuas corresponden a resultados cuanto-mecánicos y las discontinuas a resultados cuasiclásicos. La renormalización de la PDDCS implica dividir $S_{0}^{\{2\}}(\theta)$ entre $S_{0}^{\{0\}}(\theta)$, de manera que las polarizaciones intrínsecas correspondientes a distintos valores del ángulo de scattering pueden compararse entre si directamente.

cuando los productos se forman con poca excitación rotacional. Las colisiones asociadas a este mecanismo se caracterizan por grandes valores del parámetro de impacto y por poder ser tanto "nearside" como "farside" [115, 116, según sea el ángulo de deflexión positivo o negativo. La presencia de ambos tipos de colisiones ${ }^{4}$ se manifiesta a traves de la aparición de fuertes oscilaciones en las PDDCSs mecano-cuánticas para valores de $j^{\prime}$ bajos en la zona "forward" de la DCS (ver Fig. F.8). Dichas oscilaciones no pueden ser reproducidas por los cálculos de trayectorias (líneas discontinuas en la Fig. F.8) y se han atribuído a interferencias entre las colisiones "nearside" y "farside" [96.

El efecto de estas oscilaciones sobre la esterodinámica de la reacción resulta evidente al analizar los retratos estereodinámicos intrínsecos de la Fig. F.9. Debido a los bruscos cambios en los valores de las PDDCSs, su forma cambia de una manera radical con el ángulo de scattering: modificaciones de unos pocos grados cambian completamente las preferencias direccionales de la reacción.

\footnotetext{
${ }^{4}$ En contraste con el mecanismo directo, el mayoritario, donde las colisiones eran exclusivamente "nearside"
} 


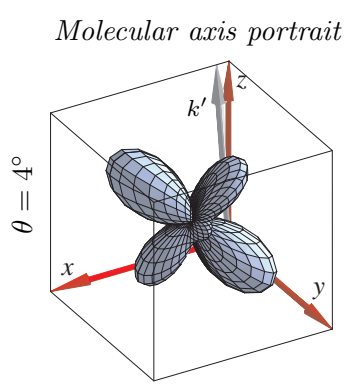

Rotational portrait
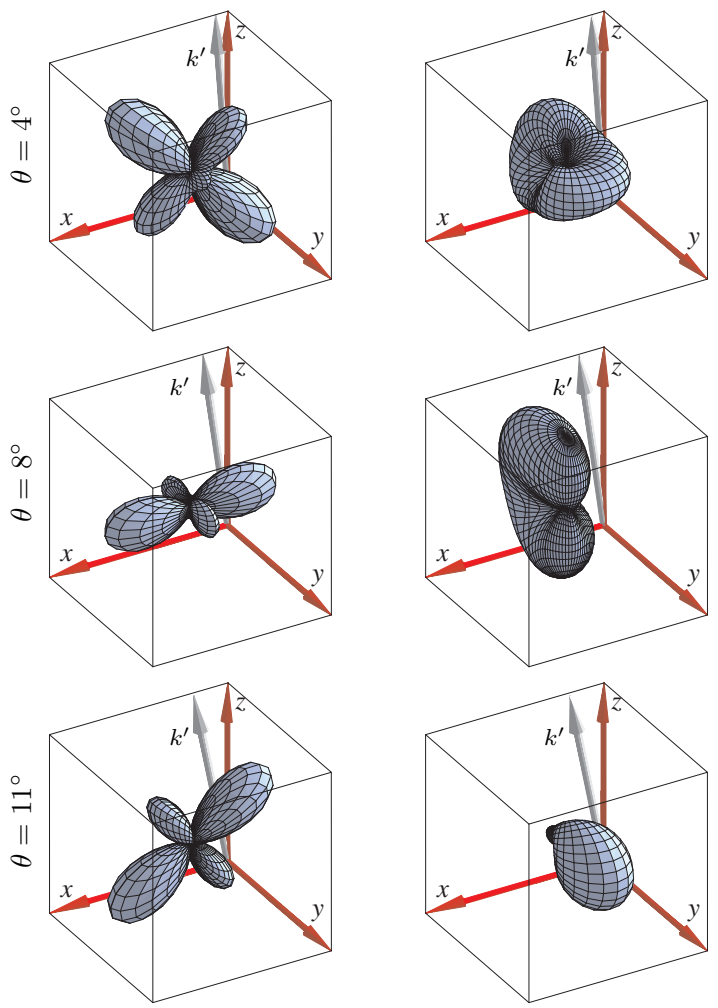

Fig. F.9.: Retratos estereodinámicos intrínsecos para la reacción $\mathrm{H}+\mathrm{D}_{2}(v=0, j=2) \rightarrow \mathrm{HD}\left(v^{\prime}=0, j^{\prime}=0\right)+\mathrm{D} \quad$ a $E_{\text {coll }}=1.306$ eV y tres valores del ángulo de scattering $\left(\theta=4,8\right.$ y $\left.11^{\circ}\right)$.

\section{F.3.3. Reacción $\mathbf{F}+\mathbf{H}_{2}(v=0, j=2)$}

La reacción $\mathrm{F}+\mathrm{H}_{2}$ es, después de la reacción $\mathrm{H}+\mathrm{H}_{2}$ y sus variantes isotópicas, la que ha recibido más atención por parte de los científicos dedicados al estudio de la dinámica molecular y constituye, por lo tanto, la elección natural a la hora de continuar el análisis de la estereodinámica de las reacciones átomo-diátomo.

Con esta finalidad, se han aplicado las técnicas expuestas en las secciones anteriores al estudio del mecanismo y del control de la reacción

$$
\mathrm{F}+\mathrm{H}_{2}(v=0, j=2) \rightarrow \mathrm{FH}\left(v^{\prime}, j^{\prime}\right)+\mathrm{H}
$$

para tres energías de colisión ${ }^{5}$ : 0.079, 0.119 y 0.148 eV. Los momentos de polarización

\footnotetext{
${ }^{5}$ Coinciden con las energías de los experimentos de haces moleculares llevados a cabo por Lee y sus
} 
intrínsecos a cada energía se han calculado a partir de las correspondientes matrices de scattering en la representación de helicidad obtenidas mediante cálculos independientes del tiempo llevados a cabo con el programa ABC 75] sobre la superficie de energía potencial desarrollada por Stark y Werner (SW) [125].

Un análisis detallado de la información contenida en los momentos intrínsecos demuestra que (i) la estereodinámica de las colisiones que llevan a $\operatorname{HF}\left(v^{\prime}=3\right)$ es diferente de la encontrada para las colisiones que llevan a $\operatorname{HF}\left(v^{\prime}=1\right.$ y 2$)$ y (ii) que se pueden distinguir dos mecanismos diferentes, que se corresponden con los mecanismos directo y retrasado encontrados al estudiar la reacción $\mathrm{H}+\mathrm{D}_{2}$. El primero continúa siendo el mecanismo dominante y muestra características fundamentalmente similares: (i) existe una clara correlación entre la excitación rotacional de los productos y la orientación de $\mathbf{j}$ a lo largo del eje y y (ii) la inclinación del eje internuclear $\mathbf{r}$ con respecto al eje $z$ tiende a ser la que facilita el acceso de los reactivos a la zona del estado de transición (que en este caso no es lineal sino plegado [125]). Esta tendencia es, sin embargo, mucho menos marcada que para la reacción $\mathrm{H}+\mathrm{D}_{2}$, posiblemente debido a que la vibración "bending" del estado de transición para la superficie SW es mucho más plana que para la superficie BKMP2, de manera que los requerimientos direccionales para acceder al estado de transición son ahora menos rigurosos.

El segundo mecanismo, el mecanismo retrasado, es únicamente discernible cuando se consideran resultados estado-a-estado resueltos en el ángulo de scattering. Las correspondientes PDDCSs presentan las mismas oscilaciones encontradas para la reacción $\mathrm{H}+\mathrm{D}_{2}$ y procedentes de interferencias entre colisiones "nearside" y "farside", con sólo dos pequeñas diferencias: (i) la amplitud de las oscilaciones es ahora menor y (ii) su presencia se extiende sobre un mayor intervalo de ángulos de scattering, indicando que, posiblemente, la vida media del complejo que da lugar al scattering "forward" es mayor para la reacción $\mathrm{F}+\mathrm{H}_{2}$ que para la $\mathrm{H}+\mathrm{D}_{2}$.

Los momentos intrínsecos pueden emplearse, además, para cuantificar el control alcanzable mediante la preparación de los reactivos. Es especialmente significativo el control sobre las secciones eficaces diferenciales, como se ilustra en las Figs. F.10 y F.11. Estas figuras contienen las DCSs correspondientes a $\operatorname{HF}\left(v^{\prime}=2\right.$ y 3$)$ y a distintos alineamientos experimentales. En ambos casos, una preparación de ejes que favorezca las colisiones colineales $\left(\beta=0^{\circ}\right)$ da lugar a la aparición de un notable máximo en la zona "backward", a $\theta=180^{\circ}$. La posición de dicho máximo se puede controlar prácticamente a voluntad, sin más que inclinar la dirección de alineamiento en el plano de scattering. Cuando esto ocurre, el máximo se desplaza suavemente hacia la zona "sideways", tanto más cuanto más se aproxime la preparación a $\beta=90^{\circ}, \alpha=0^{\circ}$,

colaboradores 120 


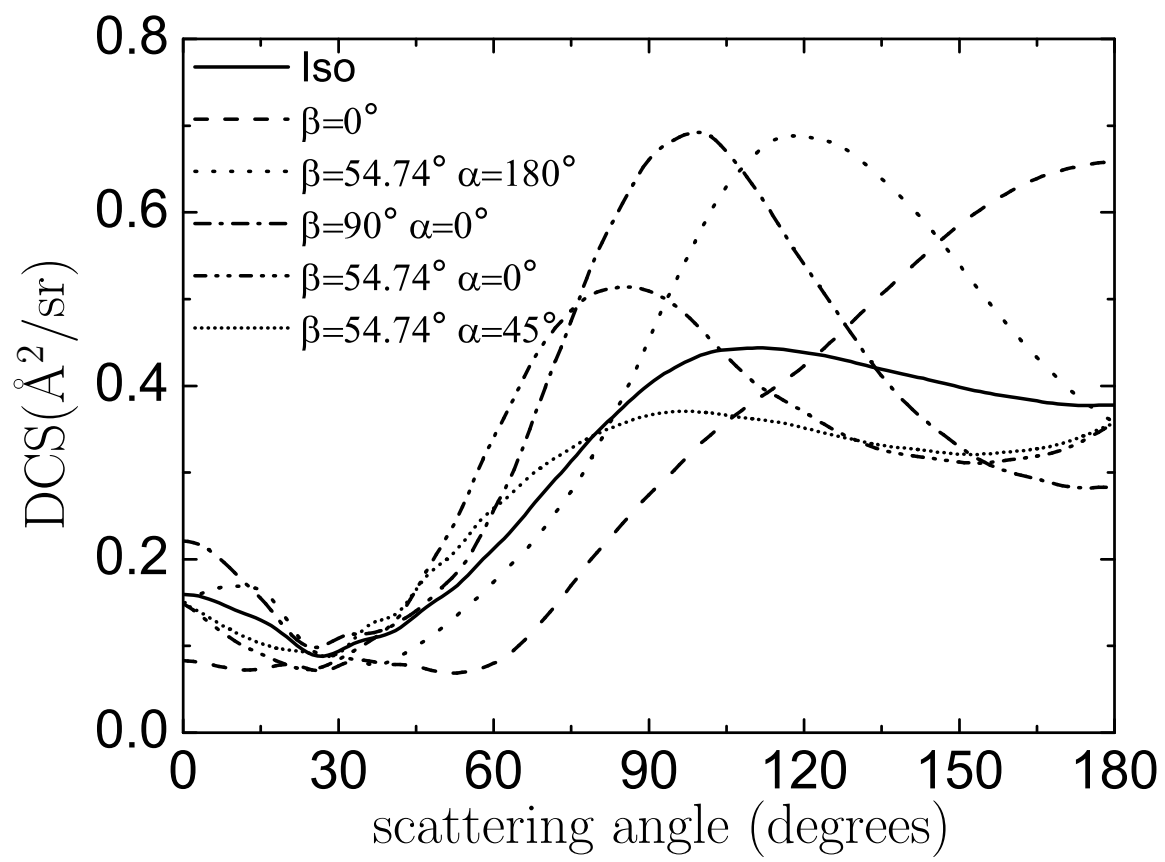

Fig. F.10.: Sección eficaz diferencial de la reacción $\mathrm{F}+\mathrm{H}_{2}(v=0, j=2) \rightarrow \mathrm{HD}\left(v^{\prime}=2, \mathrm{j}^{\prime}\right)+\mathrm{D}$ sumada sobre los niveles rotacionales finales cuando $E_{\text {coll }}=0.148 \mathrm{eV}$. Las distintas curvas corresponden a los reactivos sin polarizar (iso) y a diferentes alineamientos de la molécula $\mathrm{D}_{2}$.

permitiendo un control "a la carta" de la DCS en las zonas "backward" y "sideways". Por el contrario, la DCS "forward" muestra poca sensibilidad a la preparación de los reactivos, cambiando relativamente poco con $\alpha$ y $\beta$.

\section{F.3.4. Reacciones ultrafrías}

Se dice que una reacción está dentro del régimen ultrafrío cuando la energía de colisión es lo suficientemente baja como para que sólo una onda parcial, la que corresponde a $l=0$, sea reactiva. En estas condiciones, valores de $l \neq 0$ darían lugar a una barrera centrífuga que, aunque pequeña, sería suficiente para impedir la reacción.

Los elementos de la matriz de scattering para reacciones ultrafrías $S_{j^{\prime} \Omega^{\prime}, j \Omega}^{J}$ resultan ser independientes de la helicidad de los reactivos, es decir,

$$
S_{j^{\prime} \Omega^{\prime}, j \Omega}^{J}=S_{j^{\prime} \Omega^{\prime}, j}^{J}
$$

Este hecho influye decisivamente en los valores que los momentos de polarización 


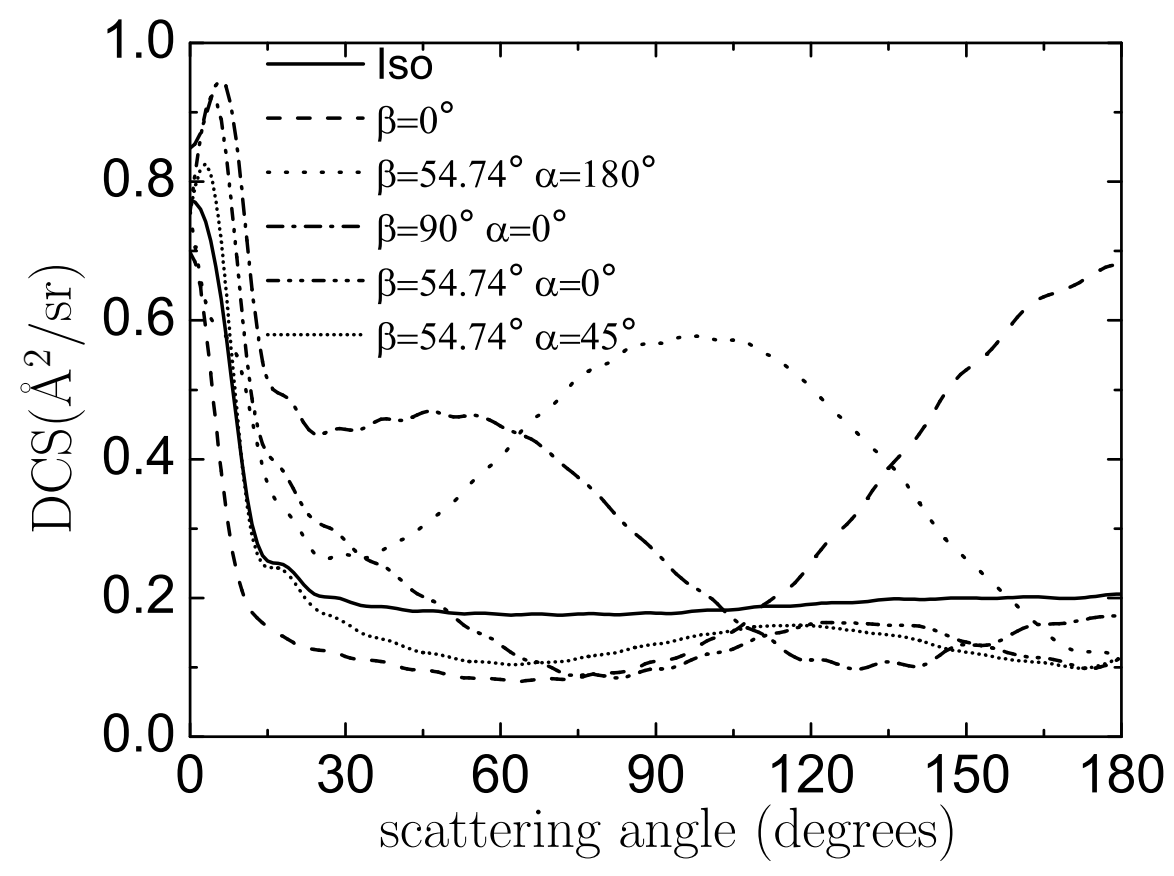

Fig. F.11.: Sección eficaz diferencial de la reacción $\mathrm{F}+\mathrm{H}_{2}(v=0, j=2) \rightarrow \mathrm{HD}\left(v^{\prime}=3, \mathrm{j}{ }^{\prime}\right)+\mathrm{D}$ sumada sobre los niveles rotacionales finales cuando $E_{\text {coll }}=0.148 \mathrm{eV}$. Las distintas curvas corresponden a los reactivos sin polarizar (iso) y a diferentes alineamientos de la molécula $\mathrm{D}_{2}$.

intrínsecos mecano-cuánticos pueden tomar cuando la energía de colisión tiende a cero y en las posibilidades de controlar la reacción. Es posible demostrar, combinando la anterior expresión con las ecuaciones (F.17) y (F.18), que

- El momento $S_{0}^{\{0\}}(\theta)$ es independiente del ángulo de scattering y toma el valor $1 / 2$.

- Todas las PDDCSs de rango ( $k$ ) impar se anulan.

- Únicamente los PPs $s_{0}^{\{0\}}$ y $s_{q}^{\{k \operatorname{par}}{ }^{2}$ ( $q$ distinto de cero) pueden tener valores no nulos. El resto serán necesariamente cero.

$\mathrm{y}$, por lo tanto,

- No es posible controlar la ICS para reacciones ultrafrías. La razón de tal imposibilidad es que el control de la ICS depende de los valores de los PPs con $q=0$ (ver $(\overline{\mathrm{F} .22})$ ), todos los cuales se anulan cuando la energía de colisión tiende a cero. 


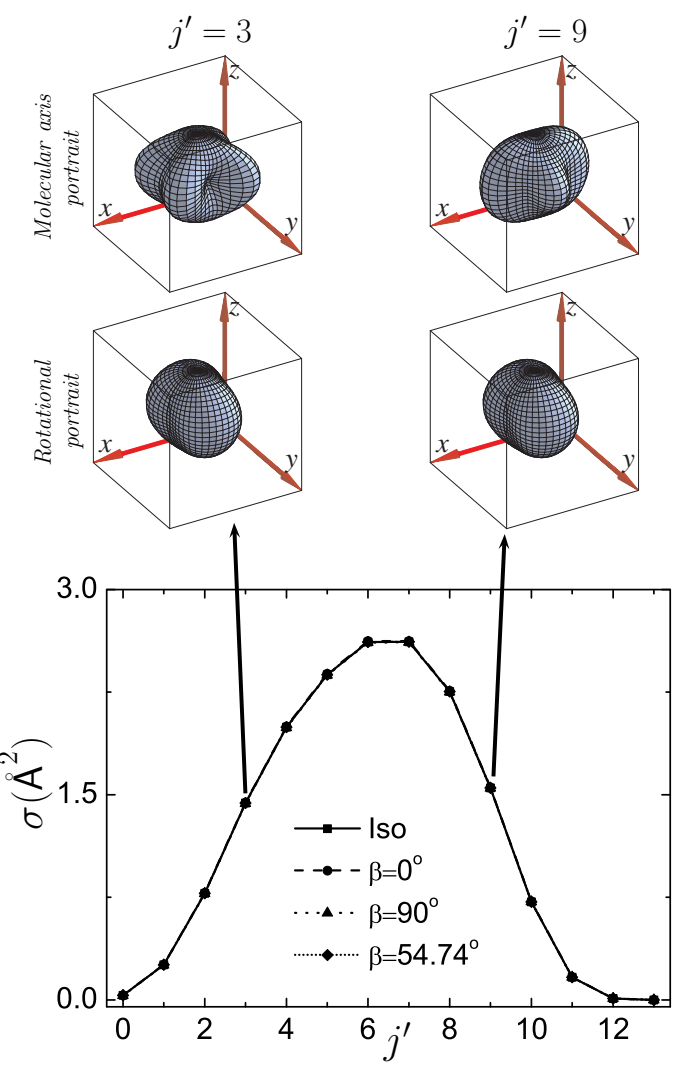

Fig. F.12.: Distribución rotacional de los productos de la reacción $\mathrm{F}+\mathrm{H}_{2}(v=0, j=2) \rightarrow \mathrm{HD}\left(v^{\prime}=2, \mathrm{j}^{\prime}\right)+\mathrm{D}$ a $E_{\text {coll }}=1 \mu \mathrm{eV}$ y retratos intrínsecos para valores seleccionados de $j^{\prime}$. Las curvas correspondientes a los reactivos sin polarizar (iso) y a los diferentes alineamientos de la molécula $\mathrm{H}_{2}$ se superponen.

- Es posible controlar la DCS para reacciones ultrafrías porque todas las PDDCSs con $k$ par pueden (y generalmente son) distintas de cero (ver $(\overline{\mathrm{F} .21})$ ).

Como ejemplo de tal control (o ausencia de él) se presentan las Figs. F.12 y F.13. En la primera se ilustra la imposibilidad de controlar la ICS de las colisiones $\mathrm{F}+\mathrm{H}_{2}(v=0$, $j=2) \rightarrow \mathrm{HF}\left(v^{\prime}=2, \mathrm{j}^{\prime}\right)+\mathrm{H}$ a $E_{\text {coll }}=1 \mu \mathrm{eV}$ (a esta energía, prácticamente sólo la onda parcial s es reactiva). Independientemente de como preparemos los reactivos no logramos modificar los valores de la ICS. Es de destacar que los retratos estereodinámicos intrísecos son muy anisotrópicos siendo, sin embargo, imposible aprovechar tal información para controlar la ICS.

La Fig.F.13 contiene mapas polares (representaciones de la DCS sobre un plano 


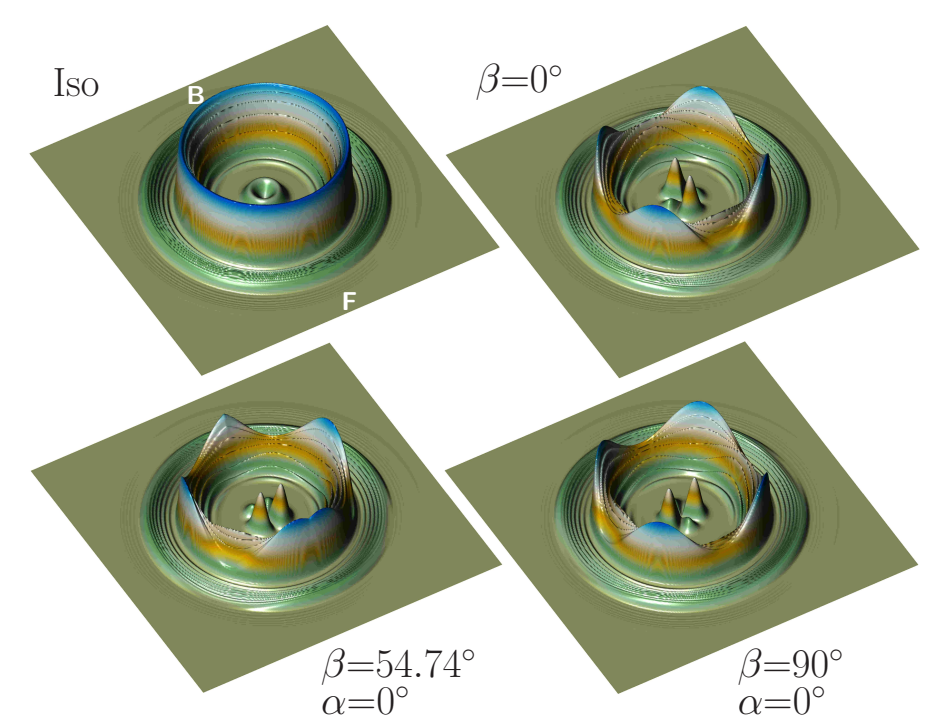

Fig. F.13.: Mapas polares para la reacción $\mathrm{F}+\mathrm{H}_{2}(v=0, j=2) \rightarrow \mathrm{HD}\left(v^{\prime}=2, \mathrm{j}^{\prime}\right)+\mathrm{D}$ a $E_{\text {coll }}=1 \mathrm{\mu eV}$. Los distintos paneles corresponden a los reactivos sin polarizar (iso) y a diferentes alineamientos de la molécula $\mathrm{H}_{2}$.

tal que el ángulo polar coincide con el ángulo de scattering y la distancia al centro es proporcional a la energía cinética de los productos, de tal manera que a mayor (menor) distancia mayor (menor) energía cinética y menor (mayor) energía interna de los productos) para la reacción $\mathrm{F}+\mathrm{H}_{2}(v=0, j=2)$ a $E_{\text {coll }}=1 \mu \mathrm{eV}$. La DCS isotrópica tiene la forma que era de esperar, es decir, es completamente isotrópica. Por su parte, las distintas preparaciones de ejes dan lugar a DCSs con formas muy distintas, destacando el hecho de que se pueden manipular independientemente las direcciones de salida de las moléculas $\operatorname{HF}\left(v^{\prime}=2\right)$ y $\operatorname{HF}\left(v^{\prime}=3\right)$.

Por ejemplo, las colisiones colineales $\left(\beta=0^{\circ}\right)$ favorecen la formación de $\operatorname{HF}\left(v^{\prime}=3\right)$ en $\theta=0$ o $180^{\circ}$, mientras que los productos $\operatorname{HF}\left(v^{\prime}=2\right)$ son dispersados de modo preferente según $\theta=45 \mathrm{o} 135^{\circ}$ (sin embargo, la distribución rotacional de los productos $\operatorname{HF}\left(v^{\prime}=2, j^{\prime}\right.$ alto $)$ sigue un patrón opuesto al adoptado por los niveles $\operatorname{HF}\left(v^{\prime}=2, j^{\prime}\right.$ bajo), donde se concentra la mayor probabilidad de reacción).

Cuando la preparación experimental es la que corresponde a $\beta=90^{\circ}, \alpha=0^{\circ}$ (colisiones laterales), la distribución angular de los productos $\operatorname{HF}\left(v^{\prime}=3\right)$ se invierte, formándose preferentemente en direcciones correspondientes a scattering "sideways". Respecto a los productos $\operatorname{HF}\left(v^{\prime}=2\right)$, la distribución angular cambia poco respecto al caso anterior para valores de $j^{\prime}$ bajos y sigue la evolución de $\mathrm{HF}\left(\mathrm{v}^{\prime}=3\right)$ para valores altos de $j^{\prime}$. 
Por último, si preparamos los reactivos según $\beta=54.74^{\circ}, \alpha=0^{\circ}$ serán las distribuciones angulares de $\operatorname{HF}\left(v^{\prime}=3\right)$ las que permanecen casi inalteradas y las asociadas a $\mathrm{HF}\left(v^{\prime}=2\right)$ las que cambian (en este caso todas rotan $45^{\circ}$ respecto al caso anterior, independientemente de si el valor de $j^{\prime}$ es alto o bajo).

\section{F.4. Conclusiones}

- Se han presentado una serie de herramientas teóricas que, basándose en el estudio de la polarización del momento angular rotacional de los reactivos en reacciones átomo-diátomo, permiten profundizar en el estudio del mecanismo de tales procesos y establecer esquemas experimentales para su control. Se ha descrito, además, un experimento que permitiría validar las predicciones teóricas.

- El formalismo matemático se ha establecido de manera que puede ser usado en combinación tanto de cálculos de trayectorias como de cálculos mecanocuánticos.

- El método se basa en la distinción entre polarización intrínseca y extrínseca de los reactivos. La primera representa su polarización cuando la reacción tiene lugar y depende exclusivamente de la dinámica del proceso. La segunda representa la preparación de reactivos correspondiente a cada experimento y, en principio, puede ser modificada a voluntad.

- En primer lugar, se han aplicado estas técnicas a la reacción $\mathrm{H}+\mathrm{D}_{2}$, encontrándose que:

- Es posible controlar los productos de reacción mediante una adecuada preparación de los reactivos. Dicho control afecta a la probabilidad de reacción, a la distribución de niveles internos de los productos y a la dirección en la que éstos se forman.

- Se pueden caracterizar dos mecanismos distintos. Uno, el mayoritario, corresponde a colisiones directas fuertemente influídas por la geometría del estado de transición y con parámetros de impacto medios y bajos. El otro, responsable del scattering "forward", corresponde a la interferencia entre colisiones "nearside" y "farside" con parámetro de impacto alto.

- El estudio de la reacción $\mathrm{F}+\mathrm{H}_{2}$ llevó a resultados cualitativamente similares: (i) es posible controlar la reacción polarizando la molécula $\mathrm{H}_{2}$ y (ii) se pueden 
diferenciar claramente los dos mismos mecanismos que para las colisiones $\mathrm{H}+\mathrm{D}_{2}$. Las estereodinámica de la reacción es diferente según que los productos sean $\operatorname{HF}\left(v^{\prime}=1\right.$ y 2$)$ o $\operatorname{HF}\left(v^{\prime}=3\right)$.

- Las técnicas desarrolladas son aplicables al estudio del mecanismo y control de reacciones químicas en régimen ultrafrío. Es estos casos, aunque el valor de la ICS no puede cambiarse polarizando los reactivos, puede modificarse notablemente la distribución angular de los productos y la DCS. 


\section{Bibliography}

[1] R. D. Levine and R. B. Berstein. Molecular Reactions Dynamics and Chemical Reactivity. Oxford University Press, Oxford, UK, 1987.

[2] J. P. Simons, Faraday Discuss., 113 (1999) 1.

[3] R. B. Bernstein, R. D. Levine and D. R. Herschbach, J. Phys. Chem., 91 (1987) 5365 .

[4] P. L. Houston, J. Phys. Chem., 100 (1996) 12757.

[5] A. J. Alexander and R. N. Zare, J. Chem. Edu., 75 (1998) 1105.

[6] R. J. Gordon and S. A. Rice, Annu. Rev. Phys. Chem., 48 (1997) 601.

[7] M. Shapiro and P. Brumer, J. Chem. Soc., Faraday Trans., 93 (1997) 1263.

[8] S. A. Rice and M.Zhao. Optical Control of Molecular Dynamics. Wiley, New York, 2000.

[9] Stereodynamics Issue, J. Phys. Chem., 91 (1987) 5365-5515.

[10] Stereodynamics Issue, J. Chem. Soc., Faraday Trans., 85 (1989) 925-1376.

[11] R. B. Bernstein Memorial Issue on Molecular Dynamics, J. Phys. Chem., 95 (1991) 7961-8421.

[12] Orientation and Polarisation Effects in Chemical Reaction Dynamics, J. Chem. Soc., Faraday Trans., 89 (1993) 1401-1592.

[13] Stereodynamics and Active Control in Chemical Reactions, J. Phys. Chem., 99 (1995) 13569-13754.

[14] Stereodynamics of Chemical Reactions, J. Phys. Chem. A, 101 (1997) 74617690. 
[15] Stereochemistry and Control in Molecular Reaction Dynamics, Faraday Discuss., 113 (1999) 1-504.

[16] Stereodynamics of Molecular Reactions, Chem. Phys., 301 (2004) 159-332.

[17] J. M. Alvarino, V. Aquilanti, S. Cavalli, S. Crocchianti, A. Laganá and M. T. Martínez, J. Phys. Chem. A, 102 (1998) 9638.

[18] M. P. de Miranda, S. Crocchianti and A. Laganá, J. Phys. Chem. A, 103 (1999) 10776 .

[19] J. M. Alvarino, F. J. Basterrechea and A. Laganá, Mol. Phys., 59 (1986) 559.

[20] H.-J. Loesch and F. Stienkemeier, J. Chem. Phys., 98 (1993) 9570.

[21] O. Hobel, and H.-J. Loesch, Faraday Discuss. Chem. Soc., 113 (1999) 337.

[22] M. Lara, A. Aguado, O. Roncero and M. Paniagua, J. Chem. Phys., 109 (1998) 9391.

[23] F. J. Aoiz, M. T. Martínez, V. Sáez-Rábanos, J. Chem. Phys., 114 (2001) 8880.

[24] M. P. de Miranda and D. C. Clary, J. Chem. Phys, 106 (1997) 4509.

[25] M. P. de Miranda, F. J. Aoiz, L. Banares and V. Saéz-Rábanos, J. Chem. Phys., 111 (1999) 5368.

[26] U. Fano, Revs. Modern Phys., 29 (1957) 74.

[27] U. Fano. Irreducible Tensorial Sets. Academic, New York, 1959.

[28] L. C. Biedenharn and J. D. Louck. Angular Momentum in Quantum Physics: Theory and Application. Addison-Wesley, New York, 1981.

[29] C. H. Greene and R. N. Zare, Annu. Rev. Phys. Chem., 33 (1982) 119.

[30] J. D. Barnwell, J. G. Loeser and D. R. Herschbach, J. Phys. Chem., 87 (1983) 2781.

[31] A. J. Orr-Ewing and R. N. Zare, Annu. Rev. Phys. Chem., 45 (1994) 315.

[32] N. E. Shafer-Ray, A. J. Orr-Ewing and R. N. Zare, J. Phys. Chem., 99 (1995) 7591.

[33] K. Blum. Density Matrix Theory and Applications. Plenum, New York, 1986. 
[34] A. Messiah. Quantum Mechanics. Dover Publications, 2000.

[35] E. M. Lifshitz and L. D. Landau. Mecánica Cuántica: Teoría no Relativista. Editorial Reverté, 1972.

[36] M. Auzinsh and R. Ferber. Optical Polarisation of Molecules. Cambridge University Press, Cambridge, UK, 1995.

[37] D. A. Varshalovich, A. N. Moskalev and V. K. Khersonskii. Quantum Theory of Angular Momentum. World Scientific, Singapore, 1988.

[38] R. N. Zare. Angular Momentum: Understanding Spatial Aspects in Chemistry and Physics. John Wiley \& Sons Inc, New York, 1987.

[39] M. P. de Miranda, F. J. Aoiz, V. Saéz-Rábanos and M. Brouard, J. Chem. Phys, 121 (2004) 9830.

[40] M. P. de Miranda and F. J. Aoiz, Phys. Rev. Lett., 93 (2004) 083201.

[41] F. J. Aoiz, M. Brouard and P. A. Enriquez, J. Chem. Phys., 105 (1996) 4964.

[42] B. D'Espagnat. Conceptual Foundations of Quantum Mechanics. Westview Press, 1999.

[43] C. Cohen-Tannoudji. Quantum Mechanics. John Wiley \& Sons Inc, New York, 1996.

[44] L. D. A. Siebbeles, M. Glass-Maujean, O. S. Vasyutinskii, J. A. Beswick and O. Roncero, J. Chem. Phys., 100 (1994) 3610.

[45] G. G. Balint-Kurti, A. J. Orr-Ewing, J. A. Beswick, Alex Brown and O. S. Vasyutinskii, J. Chem. Phys., 116 (2002) 10760.

[46] M. Hammermesh. Group Theory and its Application to Physical Problems. Dover Publications, 1990.

[47] Mary L. boas. Mathematical Methods in the Physical Sciences. John Wiley \& Sons Inc, New York, 1983.

[48] M. A. Morrison and G. A. Parker, Aust. J. Phys., 40 (1987) 465.

[49] U. Fano, J. Math. Phys., 1 (1960) 417.

[50] I. V. Hertel and W. Stoll, Adv. At. Mol. Phys., 13 (1978) 113. 
[51] R. G. Newton. Scattering Theory of Waves and Particles. Dover Publications, 2002 .

[52] J. R. Taylor. Scattering Theory. John Wiley \& Sons Inc, New York, 1972.

[53] M. L. Goldberger and K. M. Watson. Collision Theory. Dover Publications, 2004.

[54] M. Brouard. Reaction Dynamics. Oxford University Press, Oxford, UK, 1998.

[55] A. Szabo. Modern Quantum Chemistry. Cambridge University Press, Cambridge, UK, 1982.

[56] M. Born and R. Oppenheimer, Ann. Phys., 84 (1927) 457.

[57] H. Essen, Int. J. Quantum Chem., (1977) 721.

[58] D. G. Truhlar. Potential Energy Surfaces and Dynamics Calculations for Chemical Reactions and Molecular Energy Transfer. Plenum Press, New York, 1981.

[59] H. J. Monkhorst, Phys. Rev. A, 36 (1987) 1544.

[60] P. M. Kozlowski and L. Adamowicz, Chem. Rev., 93 (1993) 2007.

[61] H. Eyring, J. Chem. Phys., 3 (1935) 107.

[62] M. G. Evans and M. Polanyi, Trans. Farady Soc., 31 (1935) 875.

[63] D. G. Truhlar and B. C. Garret, Acc. Chem. Res., 13 (440) 1980.

[64] M. Karplus, R. N. Porter and R. D. Sharma, J. Chem. Phys., 43 (1965) 3259.

[65] R. B. Bernstein. Atom-Molecule Collision Theory. Plenum Press, New York, 1979.

[66] F. J. Aoiz, V. J. Herrero, and V. Sáez-Rábanos, J. Chem. Phys., 94 (1991) 7991.

[67] F. J. Aoiz, V. J. Herrero, and V. Sáez-Rábanos, J. Chem. Phys., 97 (1992) 7423 .

[68] E. M. Lifshitz and L. D. Landau. Mecánica Clásica. Editorial Reverté, 1972.

[69] W. H. Press, S. A. Teukolsky, W. T. Vetterling and B. P. Flannery. Numerical Recipies in Fortran 7\%. Cambridge University Press, Cambridge, UK, 2001. 
[70] J. J. Sakurai. Modern Quantum Mechanics. Addison-Wesley, New York, 1994.

[71] V. Aquilanti, S. Cavalli, G. Grossi and R. W. Anderson, J. Phys. Chem., 95 (1991) 8184.

[72] J. Aldegunde, J. M. Alvarino, D. de Fazio, S. Cavalli, G. Grossi and V. Aquilanti, Chem. Phys., 301 (2004) 251.

[73] M. Jacob and G. C. Wick, Annals of Physics, 7 (1959) 404.

[74] J. Z. H. Zhang and W. H. Miller, J. Chem. Phys., 91 (1989) 1528.

[75] D. Skouteris, J. F. Castillo and D. E. Manolopoulos, Comput. Phys. Commun., 133 (2000) 128.

[76] N. Balakrishnan, C. Kalyanaraman and N. Sathyamurthy, Phys. Rep., 280 (1997) 80.

[77] S. C. Althorpe and D. C. Clary, Annu. Rev. Phys. Chem., 54 (2003) 493.

[78] S. C. Althorpe, Int. Rev. Phys. Chem., 23 (2004) 219.

[79] D. E. Manolopoulos. The Encyclopedia of Computational Chemistry (State to State Reactive Scattering). John Wiley \& Sons Inc, New York, 1998.

[80] W. Hu and G. C. Schatz, J. Chem. Phys., 125 (2006) 132301.

[81] D. Kosloff and R. Kosloff, J. Comput. Phys., 52 (1983) 35.

[82] R. Kosloff, J. Phys. Chem., 92 (1988) 2087.

[83] H. Tal-Ezer and R. Kosloff, J. Chem. Phys., 81 (1984) 3967.

[84] Z. Bacic and J. C. Light, J. Chem. Phys., 85 (1986) 4594.

[85] D. Neuhauser, M. Baer, R. S. Judson and D. J. Kouri, Comput. Phys. Comm., 63 (460) 1991.

[86] W. Zhu, T. Peng and J. Z. H. Zhang, J. Chem. Phys., 106 (1997) 1742.

[87] S. C. Althorpe, J. Chem. Phys., 114 (2001) 1601.

[88] S. K. Gray and G. G. Balint-Kurti, J. Chem. Phys., 108 (1998) 950.

[89] M. Hankel, S. C. Smith, R. J. Allan, S. K. Gray and G. G. Balint-Kurti, J. Chem. Phys., 125 (2006) 164303. 
[90] D. J. Kouri and D. K. Hoffman, Few-Body Syst., 18 (1995) 203.

[91] R. Wyatt and J. Z. H. Zhang. Dynamics of Molecules and Chemical Reactions. Dekker, New York, 1996.

[92] L. M. Delves, Nuc. Phys., 9 (1959) 391.

[93] L. M. Delves, Nuc. Phys., 20 (1960) 275.

[94] F. T. Smith, J. Mat. Phys., 3 (1962) 735.

[95] W. H. Miller, Annu. Rev. Phys. Chem., 41 (1990) 245.

[96] S. C. Althorpe, F. Fernandez-Alonso, B. D. Bean, J. D. Ayers, A. E. Pomerantz, R. N. Zare and E. Wrede, Nature, 416 (2002) 67.

[97] S. A. Kandel, A. J. Alexander, Z. H. Kim, R. N. Zare, F. J. Aoiz, L. Banares, J. F. Castillo and V. Sáez-Rábanos, J. Chem. Phys., 112 (2000) 670.

[98] M. P. de Miranda, D. C. Clary, J. F. castillo and D. E. Manolopoulos, J. Chem. Phys, 108 (1998) 3142.

[99] H. J. Loesch and A. Remscheid, J. Chem. Phys., 93 (1990) 4779.

[100] B. Friedrich, D. Pullmann and D. R. Herschbach, J. Phys. Chem., 95 (1991) 8118.

[101] B. Friedrich and D. R. Herschbach, Phys. Rev. Lett., 74 (1995) 4623.

[102] G. O. Sitz and R. L. Farrow, J. Chem. Phys., 101 (1994) 4682.

[103] R. F. Code and N. F. Ramsey, Phys. Rev. A, 4 (1971) 1945.

[104] C. H. Greene and R. N. Zare, J. Chem. Phys., 78 (1983) 6741.

[105] A. J. Orr-Ewing, W. R. Simpson, T. P. Razitkis and R. N. Zare, Isr. J. Chem., 34 (1994) 95.

[106] M. Rutkowski and H. Zacharias, Chem. Phys., 301 (2004) 189.

[107] M. Rutkowski and H. Zacharias, Chem. Phys., 310 (2005) 321.

[108] L. Schnieder, K. Seekamp-Rahn, E. Wrede and K. H. Welge, J. Chem. Phys., 107 (1997) 6175.

[109] S. A. Harich, D. Dai, C. C. Wang, X. Yang, S. D. Chao and R. Skodje, Nature, 419 (2002) 281. 
[110] F. J. Aoiz, L. Banares and V. J. Herrero, Int. Rev. Phys. Chem., 24 (2005) 119.

[111] A. I. Boothroyd, W. J. Keogh, P. G. Martin and M. R. Peterson, J. Chem. Phys., 104 (1996) 7139.

[112] B. K. Kendrick, J. Chem. Phys., 114 (2001) 8796.

[113] R. D. Levine, J. Phys. Chem., 94 (1990) 8872.

[114] S. C. Althorpe, J. Chem. Phys., 117 (2002) 4623.

[115] A. J. Dobbyn, P. McCabe, J. N. L. Connor and J. F. Castillo, Phys. Chem. Chem. Phys., 1 (1999) 1115.

[116] C. Noli, J. N. L. Connor, N. Rougeau and C. Kubach, Phys. Chem. Chem. Phys., 3 (2001) 3946.

[117] F. J. Aoiz, L. Banares, J. F. Castillo and D. Sokolovski, J. Chem. Phys., 117 (2002) 2546.

[118] F. J. Aoiz, V. Saéz-Rábanos, B. Martínez-Haya and T. González-Lezana, J. Chem. Phys., 123 (2005) 094101.

[119] A. N. Panda and S. C. Althorpe, Chem. Phys. Lett., 419 (2006) 245.

[120] D. M. Neumark, A. M. Wodtke, G. N. Robinson, C. C. Hayden and Y. T. Lee, J. Chem. Phys., 82 (1985) 3045.

[121] D. E. Manolopoulos, J. Chem. Soc., Faraday Trans., 93 (1997) 673.

[122] R. B. Metz, S. E. Bradforth and D. M. Neumark, Adv. Chem. Phys., 81 (1992) 1.

[123] D. M. Neumark, Annu. Rev. Phys. Chem., 43 (1992) 153.

[124] D. M. Neumark, Acc. Chem. Phys., 26 (1993) 33.

[125] K. Stark and H.-J. Werner, J. Chem. Phys., 104 (1996) 6515.

[126] D. E. Manolopoulos, K. Stark, H.-J. Werner, D. W. Arnold, S. E. Bradforth and D. M. Neumark, Science, 262 (1993) 1852.

[127] F. J. Aoiz, L. Banares, V. J. Herrero, V. Saéz-Rábanos, K. Stark and H.-J. Werner, Chem. Phys. Lett., 223 (1994) 215. 
[128] F. J. Aoiz, L. Banares, V. J. Herrero, V. Saéz-Rábanos, K. Stark and H.-J. Werner, J. Chem. Phys., 102 (1995) 9248.

[129] J. F. Castillo, D. E. Manolopoulos, K. Stark and H.-J. Werner, J. Chem. Phys, $104(1996) 6531$.

[130] F. J. Aoiz, L. Banares, B. Martínez-Haya, J. F. Castillo, D. Manolopoulos ,K. Stark and H.-J. Werner, J. Phys. Chem. A, 101 (1997) 6403.

[131] G. C. Schatz, J. M. Bowman and A. Kuppermann, J. Chem. Phys, 58 (1973) 4023.

[132] R. T. Skodje, D. Skouteris, D. E. Manolopoulos, S.-H. Lee, F. Dong and K. Liu, Phys. Rev. Lett., 85 (2000) 1206.

[133] S. D. Chao and R. T. Skodje, J. Chem. Phys., 113 (2000) 3487.

[134] M. Qiu, Z. Ren, L. Chen et al, Science, 311 (2006) 1440.

[135] Z. Ren, Li Che, M. Qiu et al, J. Chem. Phys., 125 (2006) 151102.

[136] F. J. Aoiz, M. Brouard, V. J. Herrero, V. Saéz-Rábanos, and K. Stark, Chem. Phys. Lett., 264 (1997) 487.

[137] Althorpe S. C., J. Phys. Chem. A, 107 (2003) 7152.

[138] R. V. Krems, Int. Rev. Phys. Chem., 24 (2005) 99.

[139] J. Doyle, B. Friedrich, R. V. Krems and F. Masnou-Seeuws, Eur. Phys. J. D, 31 (2004) 149.

[140] A. Volpi and J. L. Bohn, Phys. Rev. A, 65 (2002) 052712.

[141] A. V. Avdeenkov and J. L. Bohn, Phys. Rev. Lett., 90 (2003) 043006.

[142] R. V. Krems and A. Dalgarno, Phys. Rev. A, 67 (2003) 050704.

[143] R. V. Krems and A. Dalgarno, Phys. Rev. A, 68 (2003) 013406.

[144] A. V. Avdeenkov, D. C. E. Bortolotti and J. L. Bohn, Phys. Rev. A, 69 (2004) 012710 .

[145] E. Bodo, F. A. Gianturco, N. Balakrishnan and A. Dalgarno, J. Phys. B, 37 (2004) 3641.

[146] R. V. Krems, Phys. Rev. Lett., 93 (2004) 013201. 
[147] E. Bodo and F. A. Gianturco, Eur. Phys. J. D, 31 (2004) 423.

[148] E. P. Wigner, Phys. Rev., 73 (1948) 1002.

[149] N. Balakrishnan and A. Dalgarno, Chem. Phys. Lett., 341 (2001) 652.

[150] M. Abramowitz and I. A. Stegun. Handbook of Mathematical Functions. Dover Publications, 1972.

[151] E. Bodo, F. A. Gianturco, and A. Dalgarno, J. Phys. B, 35 (2002) 2391.

[152] N. Balakrishnan and A. Dalgarno, J. Phys. Chem. A, 107 (2003) 7101. 